\title{
Design and Synthesis of Photodissociable Ligands (PDL) Based on Azoimidazoles for Light-Driven Coordination-Induced Spin State Switching (LD- CISSS) in Homogeneous Solution.
}

\author{
Christian Schütt, Gernot Heitmann, Thore Wendler, \\ Bahne Krahwinkel and Rainer Herges*
}

\section{Table of Contents}

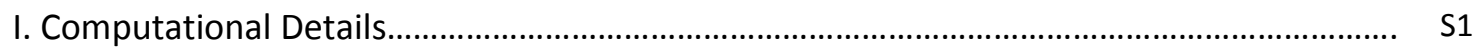

I.1 1-Substituted 5-phenylazoimidazoles...................................................................... S1

$1.2 \quad$ I.2 Calculated UV Spectra................................................................................ S2

I.3 Calculated Complex Formation Enthalpy of 1-Methylimidazole with NiTPPF $20 \ldots \ldots .$. S4

I.4 Effect of Substituents at the Phenyl Ring on the Complex Formation Enthalpies.... S7

I.5 XYZ Coordinates of the PBE/SVP optimized Ligands.............................................. S8

I.6 XYZ Coordinates of the PBE/SVP optimized Complexes........................................... S26

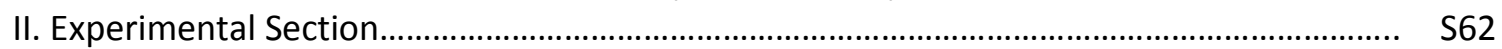

II.1 Materials and Methods......................................................................................... S62

II.2 NMR Spectroscopy..................................................................................... S63

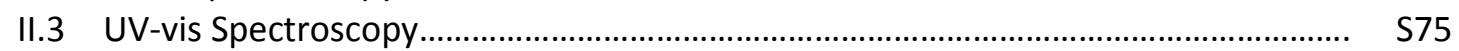

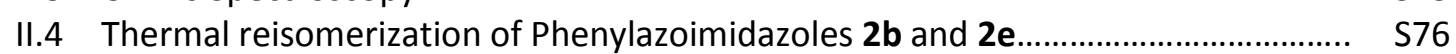

II.5 Association Constants and Thermodynamic Parameters......................................... S S77

II.6 NMR spin switching experiments...................................................................... S82

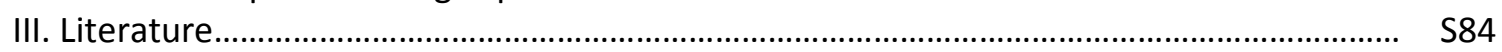




\section{Computational Details}

\section{I.1 1-Substituted 5-phenylazoimidazoles}

The calculations have been performed using Turbomole $6.3 .{ }^{1}$ The geometry optimizations have been carried out at the PBE/SVP level of theory.

Table S1. Calculated (PBE/SVP) relative energies of the cis- and trans-isomers of 1-substituted-5phenylazoimidazoles and their respective conformers, relative to the trans $\beta$ isomer.

\begin{tabular}{|c|c|c|c|}
\hline Ligand & Isomer & $\begin{array}{c}E_{\text {abs }} \\
\text { [Hartree] }\end{array}$ & $\begin{array}{c}\mathrm{E}_{\mathrm{rel}} \\
{[\mathrm{kcal} / \mathrm{mol}]}\end{array}$ \\
\hline \multirow[t]{4}{*}{1} & cis- $\alpha$ & -565.61636046 & 14.78 \\
\hline & cis- $\beta$ & -565.61656729 & 14.65 \\
\hline & trans- $\alpha$ & -565.63559816 & 2.71 \\
\hline & trans- $\beta$ & -565.63991806 & 0.00 \\
\hline \multirow[t]{4}{*}{2} & $\operatorname{cis}-\alpha$ & -604.84043867 & 12.21 \\
\hline & $\operatorname{cis}-\beta$ & -604.83179640 & 17.63 \\
\hline & trans- $\alpha$ & -604.85951027 & 0.24 \\
\hline & trans- $\beta$ & -604.85989322 & 0.00 \\
\hline \multirow[t]{4}{*}{3} & cis- $\alpha$ & -683.29354830 & 10.81 \\
\hline & $\operatorname{cis}-\beta$ & -683.28233428 & 17.85 \\
\hline & trans- $\alpha$ & -683.31235786 & -0.99 \\
\hline & trans- $\beta$ & -683.31100720 & 0.00 \\
\hline \multirow[t]{4}{*}{4} & $\operatorname{cis}-\alpha$ & -973.79344593 & 12.17 \\
\hline & $\operatorname{cis}-\beta$ & -973.77805887 & 21.83 \\
\hline & trans- $\alpha$ & -973.81151982 & 0.83 \\
\hline & trans- $\beta$ & -973.81284143 & 0.00 \\
\hline \multirow[t]{4}{*}{5} & $\operatorname{cis}-\alpha$ & -722.51420713 & 10.36 \\
\hline & cis- $\beta$ & -722.49616539 & 21.68 \\
\hline & trans- $\alpha$ & -722.53368385 & -1.86 \\
\hline & trans- $\beta$ & -722.53072157 & 0.00 \\
\hline
\end{tabular}

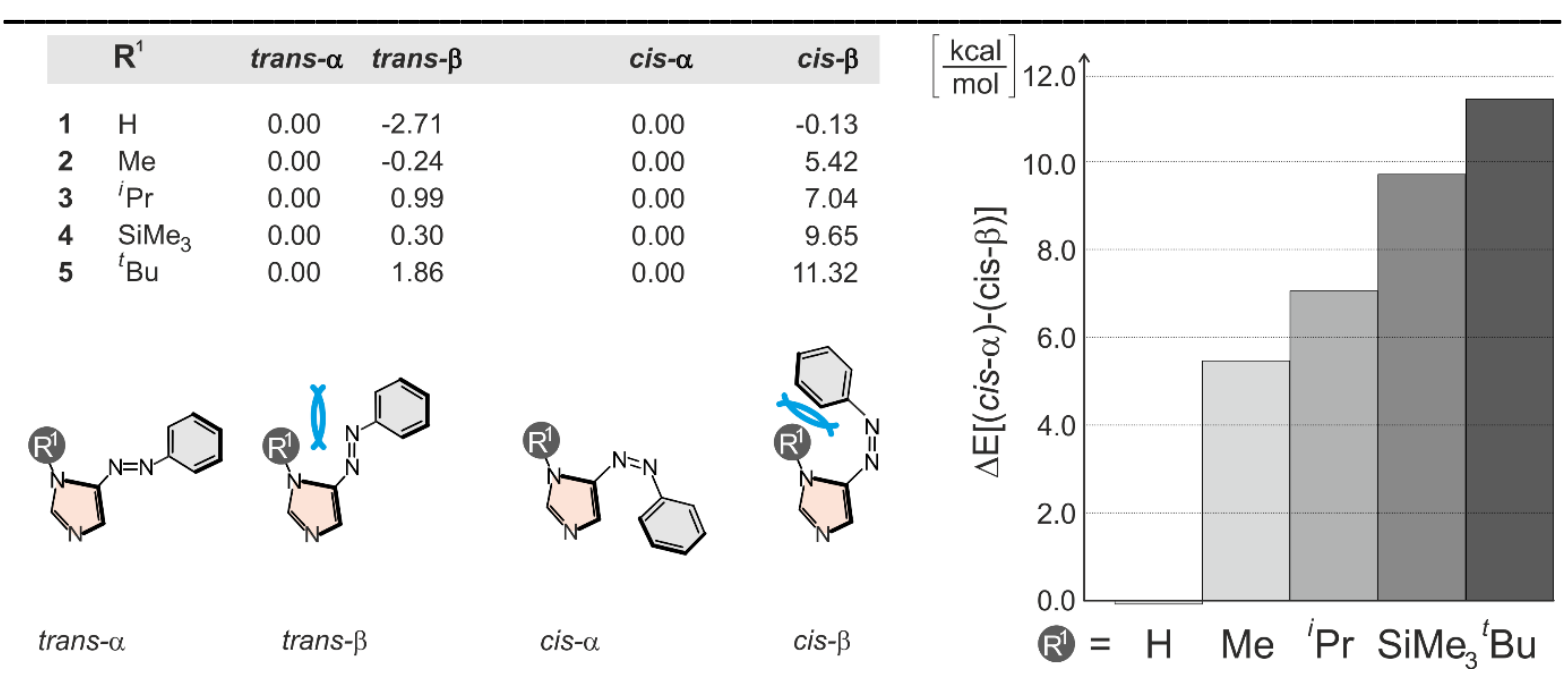

Figure S1. Energy differences $(\Delta E)$ of the $\alpha$ and $\beta$ conformer of the trans and cis isomer relative to the respective $\alpha$ conformation (left). Bar diagram of the energy difference of the two cis conformers in $\mathrm{kcal} \cdot \mathrm{mol}^{-1}(\Delta E)$ (right). Intramolecular sterical hindrance is indicated by blue arcs. 


\section{I.2 Calculated UV Spectra}

The TD-DFT calculations of the 1-methyl-5-phenylazoimidazole (2) and its corresponding derivatives 2a-c and $2 \mathbf{e}$ were performed at the B3LYP/6-31G* level of theory using Gaussian 09.D01. ${ }^{2}$ The calculated wavelengths of the transitions predicted by the TD-DFT calculations deviate systematically by $10-30 \mathrm{~nm}$ with respect to our experimental UV-VIS spectra. ${ }^{3}$
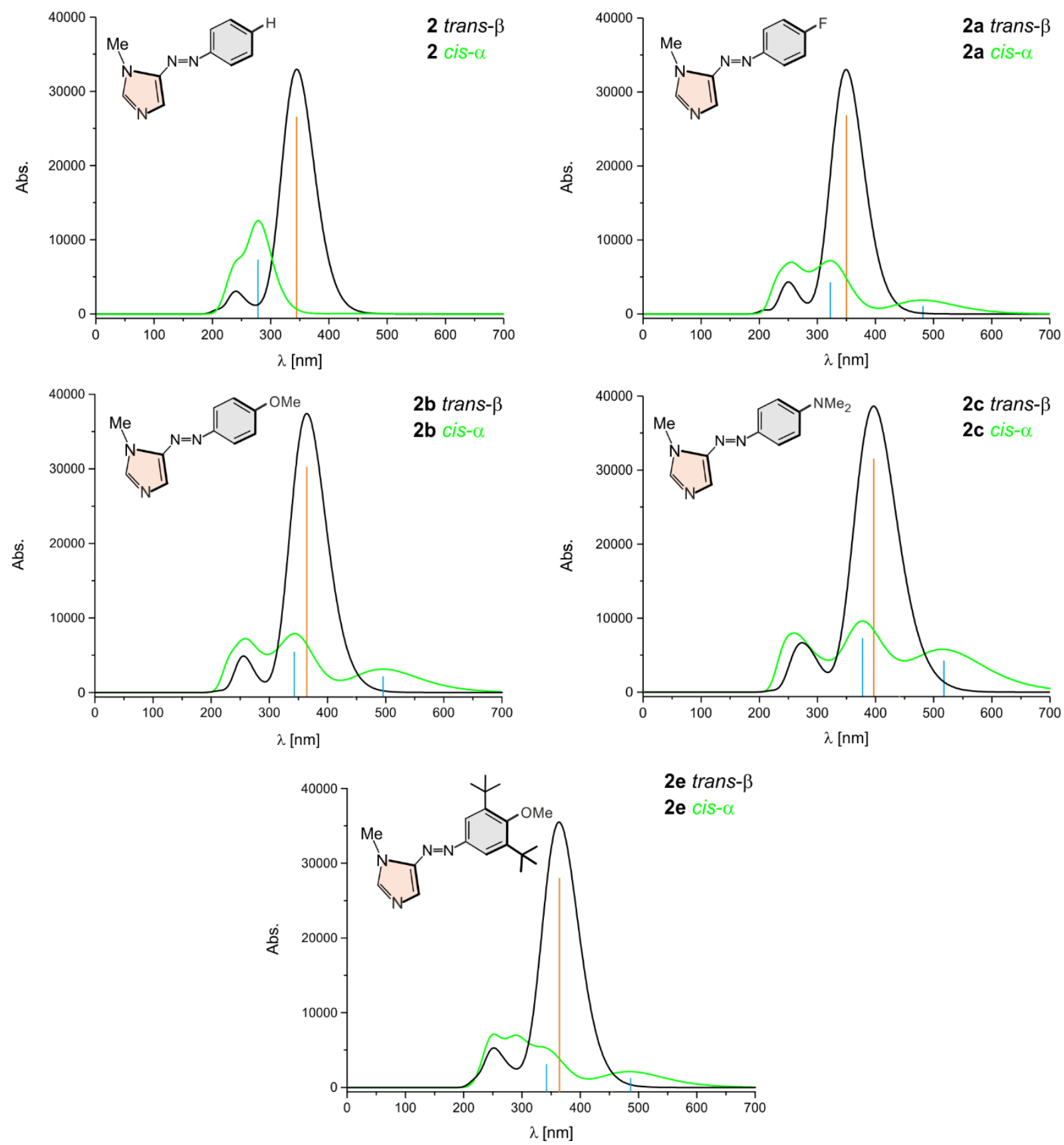

Figure S2. Calculated (TD-B3LYP/6-31G*) UV spectra of the most stable conformation of the trans (black) and cis (green) isomer of the parent system $\mathbf{2}$ (top left) and its corresponding derivatives $\mathbf{2 a}$ (top right), $\mathbf{2 b}$ (middle left), $\mathbf{2 c}$ (middle right) and $\mathbf{2 e}$ (bottom). The orange (trans isomer) and cyan (cis isomer) bars represent the $\pi-\pi^{*}$ and $n-\pi^{*}$ transitions, the height of the bars is proportional to the oscillator strength. The UV spectra are simulated by an overlay of Gaussian functions with a half-width of $0.333 \mathrm{eV}$. 
According to the calculations the photochemical isomerization from trans to cis should be efficient in $\mathbf{2}, \mathbf{2} \mathbf{a}$ and $\mathbf{2} \mathbf{b}$, because there is little overlap of the $\pi-\pi^{*}$ transition of the trans with transitions of the cis form. In $\mathbf{2 c}$, however, the $\pi-\pi^{*}$ bands of trans and cis exhibit considerable overlap, therefore an inefficient trans $\rightarrow$ cis switching is predicted. The calculations predict a very low oscillator strength for the $\mathrm{n}-\pi^{*}$ transition of the parent system in trans as well as in cis configuration. Hence, back-switching (cis $\rightarrow$ trans) should be hampered in the parent system, which leaves the fluoro- and methoxy substituted systems $\mathbf{2} \mathbf{a}$ and $\mathbf{2} \mathbf{b}$ as viable candidates. The methoxy substituted system $\mathbf{2} \mathbf{b}$ is superior because it combines a good separation between the $\pi-\pi^{*}$ transition of the trans form and the $n-\pi^{*}$ transition of the cis configuration with a high oscillator strength of the $\mathrm{n}-\pi^{*}$ transition in the cis form (for details see Table S2). Introduction of two $t$-butyl groups at the phenyl ring (2e) leads to small hypsochromic shift of the vital transitions of the PDL in comparison to $\mathbf{2 b}$. Nevertheless, $\mathbf{2}$ e exhibits a good separation between the $\pi-\pi^{*}$ transition of the trans form and the $n-\pi^{*}$ transition of the cis isomer maintaining a high oscillator strength of the $n-\pi^{*}$ transition in the cis configuration.

Table S2. Calculated (TD-B3LYP/6-31G*) transitions of the most stable cis- and trans-isomers of 1-methyl-5-phenylazoimidazole (2) and its derivatives $\mathbf{2 a}, \mathbf{2 b}, \mathbf{2 c}$ and $\mathbf{2 e}$.

\begin{tabular}{|l|l|l|l|l|}
\hline \multicolumn{1}{|c|}{$\#$} & \multicolumn{1}{|c|}{ Isomer } & $\boldsymbol{\lambda}[\mathrm{nm}]$ & \multicolumn{1}{|c|}{$\boldsymbol{F}$} & \multicolumn{1}{|c|}{ Transition $\left.{ }^{2}\right)$} \\
\hline $\mathbf{2}$ & trans- $\beta$ & 345 & 0.809 & $\pi-\pi^{*}$ \\
\hline & & 456 & 0.000 & $\mathrm{n}-\pi^{*}$ \\
\hline & cis- $\alpha$ & 279 & 0.248 & $\pi-\pi^{*}$ \\
\hline $\mathbf{2 a}$ & & 451 & 0.001 & $\mathrm{n}-\pi^{*}$ \\
\hline & trans- $\beta$ & 350 & 0.815 & $\pi-\pi^{*}$ \\
\hline & & 449 & 0.000 & $\mathrm{n}-\pi^{*}$ \\
\hline & cis- $\alpha$ & 331 & 0.101 & $\mathrm{n}-\pi^{*}\left(\pi-\pi^{*}\right)$ \\
\hline $\mathbf{2 b}$ & & 470 & 0.033 & $\mathrm{n}-\pi^{*}\left(\pi-\pi^{*}\right)$ \\
\hline & trans- $\beta$ & 364 & 0.924 & $\pi-\pi^{*}$ \\
\hline & & 444 & 0.000 & $\mathrm{n}-\pi^{*}$ \\
\hline & cis- $\alpha$ & 349 & 0.177 & $\mathrm{n}-\pi^{*}\left(\pi-\pi^{*}\right)$ \\
\hline $\mathbf{2 c}$ & & 495 & 0.077 & $\mathrm{n}-\pi^{*}\left(\pi-\pi^{*}\right)$ \\
\hline & trans- $\beta$ & 397 & 0.954 & $\pi-\pi^{*}$ \\
\hline & & 442 & 0.001 & $\mathrm{n}-\pi^{*}$ \\
\hline & cis- $\alpha$ & 378 & 0.235 & $\mathrm{n}-\pi^{*}\left(\pi-\pi^{*}\right)$ \\
\hline $\mathbf{2 e}$ & & 519 & 0.142 & $\mathrm{n}-\pi^{*}\left(\pi-\pi^{*}\right)$ \\
\hline & trans- $\beta$ & 364 & 0.865 & $\pi-\pi^{*}$ \\
\hline & & 452 & 0.000 & $\mathrm{n}-\pi^{*}$ \\
\hline & cis- $\alpha$ & 345 & 0.109 & $\mathrm{n}-\pi^{*}\left(\pi-\pi^{*}\right)$ \\
\hline & & 484 & 0.052 & $\mathrm{n}-\pi^{*}\left(\pi-\pi^{*}\right)$ \\
\hline
\end{tabular}

a) An unequivocal assignment of excitations to $\pi-\pi^{*}$ or $n-\pi^{*}$ is not possible because $\pi$ and $n$ orbitals are not exactly orthogonal in the cis configurations. The major contributions are given in the Table and the minor contributions are given in parentheses. 


\section{I.3 Calculated Complex Formation Energy of 1-Methylimidazole with NiTPPF 20}

The geometry optimizations of the five and six coordinated NiTPPF $_{20}$ complexes with 1-methylimidazole (1-MeIm) were performed at the PBE/SVP level of density functional theory. Single point energies were calculated at the optimized geometries applying several functionals and basis sets using Gaussian 09. The data (Table S3 and Figure S3) confirm that the B3LYP/def2TZVP//PBE/SVP level of theory is a good compromise between computational costs and accuracy. The calculated complex formation energies $\left(\Delta E_{f}\right)$ of the five and six coordinated complex with NiTPPF 20 are compared with the corresponding experimentally determined ( $\left.\Delta H_{\text {expt.1}}\right)$ values (see section II.5).

Table S3. Calculated energies for the formation of the five coordinate ( $\Delta E_{\mathrm{f}}$ one axial ligand) and six coordinate ( $\triangle E_{f}$ two axial ligands) complexes of $\mathrm{NiTPPF}_{20}$ with 1-methylimidazole, according to the following equations: : $\Delta E_{\mathrm{f}}\left(\right.$ 5-coord.) : NiTPPF 20 (singlet) + Melm $\rightarrow \mathrm{NiTPPF}_{20} \cdot \mathrm{Melm}$ (triplet) $\Delta E_{\mathrm{f}}(6$-coord. $): \mathrm{NiTPPF}_{20}($ singlet $)+2 \mathrm{Melm} \rightarrow \mathrm{NiTPPF}_{20} \cdot 2 \mathrm{Melm}$ (triplet)

\begin{tabular}{|c|c|c|c|c|c|c|c|}
\hline Functional & Basis set & $\begin{array}{c}\text { Eabs } \\
\text { 1-Melm } \\
\text { [a.u.] }\end{array}$ & $\begin{array}{c}E_{\text {abs }} \\
\text { NiTPPF }_{20} \text { (singlet) } \\
\text { [a.u.] }\end{array}$ & $\begin{array}{c}\text { Eabs } \\
\text { NiTPPF }_{20} \cdot \text { Melm } \\
\text { (triplet) [a.u.] }\end{array}$ & $\begin{array}{c}E_{a b s} \\
\text { NiTPPF }_{20} \cdot 2 \mathrm{Melm} \\
\text { (triplet) [a.u.] }\end{array}$ & $\begin{array}{c}\Delta E_{\mathrm{f}} \\
\text { 5-coord. } \\
{[\mathrm{kcal} / \mathrm{mol}]}\end{array}$ & $\begin{array}{c}\Delta E_{f} \\
\text { 6-coord. } \\
{[\mathrm{kcal} / \mathrm{mol}]}\end{array}$ \\
\hline PBE & SVP & -265.0101862 & -5398.2568650 & -5663.2798821 & -5928.3148331 & -8.05 & -23.59 \\
\hline PBE & def2TZVP & -265.2980412 & -5402.8891357 & -5668.1864973 & -5933.5000643 & 0.43 & -9.32 \\
\hline PBE & 6-31G* & -265.1983126 & -5401.0648635 & -5666.2697842 & -5931.4909873 & -4.15 & -18.51 \\
\hline PBE & 6-31+G* & -265.2104974 & -5401.3237703 & -5666.5373792 & -5931.7677293 & -1.95 & -14.41 \\
\hline PBE & 6-311G* & -265.2547987 & -5402.2047107 & -5667.4679998 & -5932.7473535 & -5.33 & -20.74 \\
\hline PBEO & SVP & -265.0286380 & -5398.2512105 & -5663.3211890 & -5928.3745799 & -25.74 & -41.47 \\
\hline PBEO & def2TZVP & -265.3129806 & -5402.8745546 & -5668.2155754 & -5933.5449613 & -17.60 & -27.89 \\
\hline PBEO & 6-31G* & -265.2166579 & -5401.1095675 & -5666.3622832 & -5931.6012891 & -22.63 & -36.65 \\
\hline PBEO & 6-31+G* & -265.2264723 & -5401.3212005 & -5666.5786260 & -5931.8259368 & -19.42 & -32.50 \\
\hline PBEO & 6-311G* & -265.2693873 & -5402.1828943 & -5667.4903560 & -5932.7840898 & -23.89 & -39.17 \\
\hline B3LYP & SVP & -265.3355052 & -5402.5025914 & -5667.8676603 & -5933.2218140 & -18.55 & -30.25 \\
\hline B3LYP & def2TZVP & -265.6288445 & -5407.1843308 & -5672.8291203 & -5938.4677819 & -10.10 & -16.17 \\
\hline B3LYP & 6-31G* & -265.5259460 & -5405.3375140 & -5670.8881057 & -5936.4306552 & -15.47 & -25.88 \\
\hline B3LYP & 6-31+G* & -265.5380757 & -5405.5828883 & -5671.1393098 & -5936.6907076 & -11.51 & -19.87 \\
\hline B3LYP & 6-311G* & -265.5849436 & -5406.4938291 & -5672.1047572 & -5937.7076955 & -16.31 & -27.60 \\
\hline MN12L & SVP & -265.0581622 & -5399.3187902 & -5664.3876208 & -5929.4844044 & -6.69 & -30.93 \\
\hline MN12L & def2TZVP & -265.3522601 & -5404.0980455 & -5669.4416754 & -5934.8212210 & 5.42 & -11.71 \\
\hline MN12L & 6-31G* & -265.2584960 & -5402.3841366 & -5667.6458856 & -5932.9405982 & -2.04 & -24.77 \\
\hline MN12L & 6-31+G* & -265.2669770 & -5402.5875865 & -5667.8549774 & -5933.1563276 & -0.26 & -21.83 \\
\hline MN12L & 6-311G* & -265.3031440 & -5403.4217338 & -5668.7257112 & -5934.0670213 & -0.52 & -24.47 \\
\hline MN12SX & SVP & -265.0822315 & -5399.4116786 & -5664.5207329 & -5929.6377437 & -16.83 & -38.66 \\
\hline MN12SX & def2TZVP & -265.4050773 & -5404.5373742 & -5669.9538100 & -5935.3840315 & -7.13 & -22.91 \\
\hline MN12SX & 6-31G* & -265.3060581 & -5402.7451774 & -5668.0732325 & -5933.4114938 & -13.80 & -34.01 \\
\hline MN12SX & $6-31+G^{*}$ & -265.3161113 & -5402.9504155 & -5668.2842261 & -5933.6298726 & -11.11 & -29.64 \\
\hline MN12SX & 6-311G* & -265.3562694 & -5403.8129199 & -5669.1898686 & -5934.5809836 & -12.98 & -34.84 \\
\hline TPSSh & SVP & -265.3591214 & -5402.6125677 & -5667.9997498 & -5933.3819689 & -17.61 & -38.10 \\
\hline TPSSh & def2TZVP & -265.6431682 & -5407.2468389 & -5672.9048322 & -5938.5625278 & -9.30 & -18.42 \\
\hline TPSSh & 6-31G* & -265.5449205 & -5405.4465224 & -5671.0143717 & -5936.5803601 & -14.39 & -27.61 \\
\hline TPSSh & $6-31+G^{*}$ & -265.5547181 & -5405.6636485 & -5671.2369181 & -5936.8106801 & -11.64 & -23.59 \\
\hline TPSSh & 6-311G* & -265.6006346 & -5406.5601912 & -5672.1852690 & -5937.8088192 & -15.34 & -29.72 \\
\hline \multicolumn{2}{|c|}{ Experiment $\left(\Delta H_{\text {exptl. }}\right)$} & & & & & $\begin{array}{c}-7.27 \\
( \pm 0.37)\end{array}$ & $\begin{array}{c}-13.86 \\
( \pm 0.67)\end{array}$ \\
\hline
\end{tabular}


$\mathrm{NiTPPF}_{20} \cdot 1$ Melm:

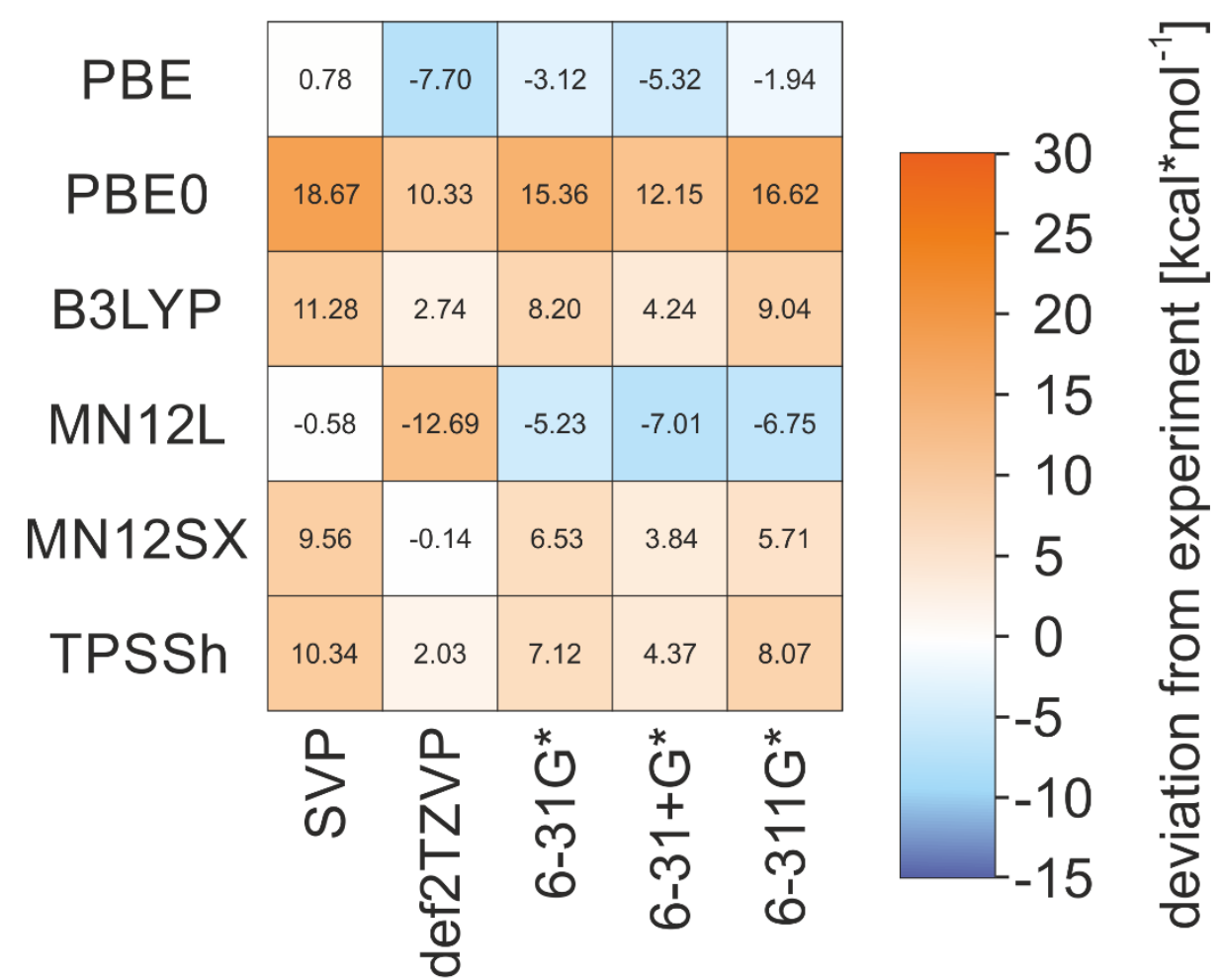

$\mathrm{NiTPPF}_{20} \cdot 2$ Melm:

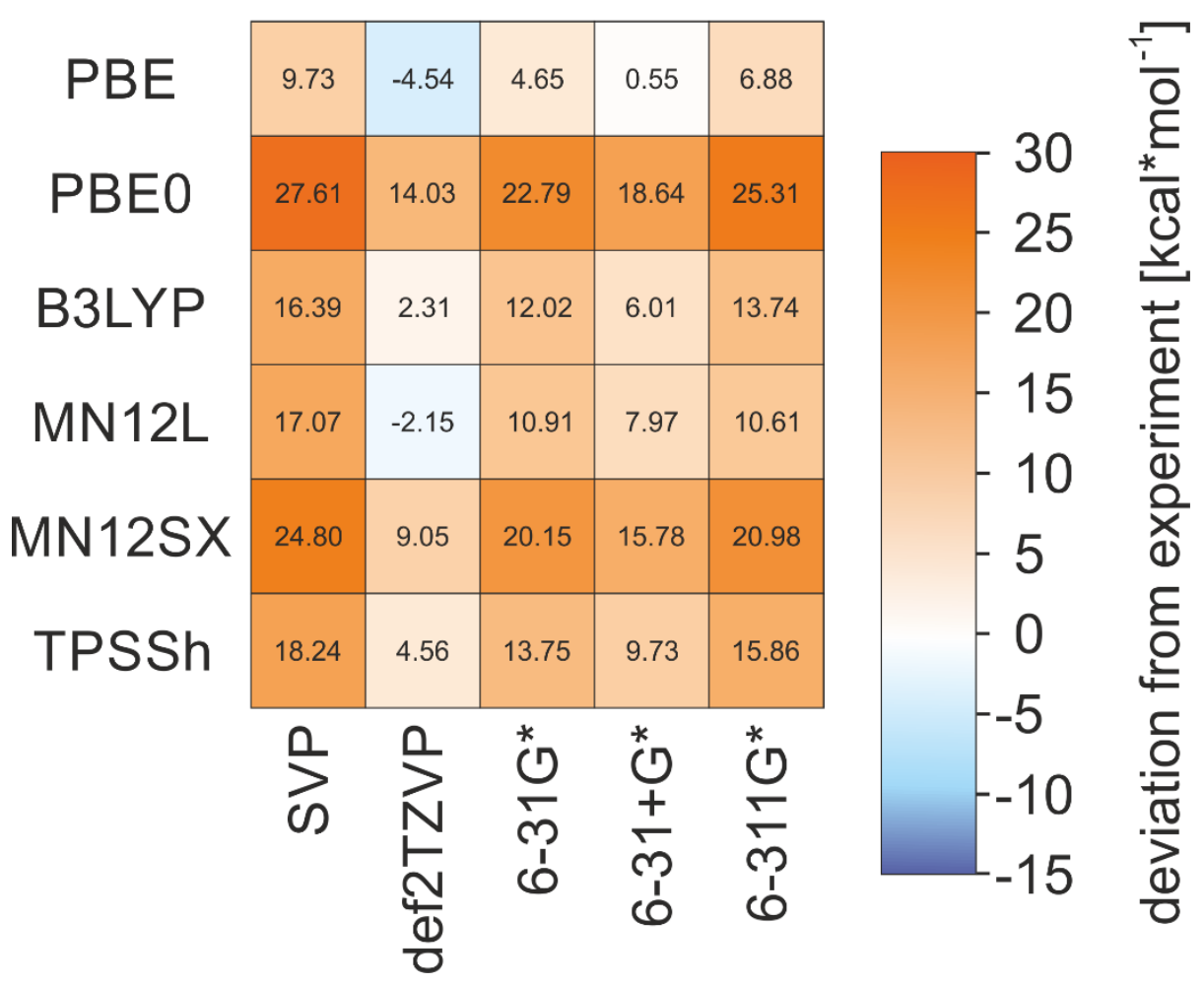

Figure S3. Color coded representation of the deviation of theoretically calculated and experimentally determined values for the formation of the five- (top) and six coordinate (bottom) complex of NiTPPF 20 with on and two methylimidazole molecules at different levels of theory. The exact values are given in $\mathrm{kcal}^{*} \mathrm{~mol}^{-1}$. 


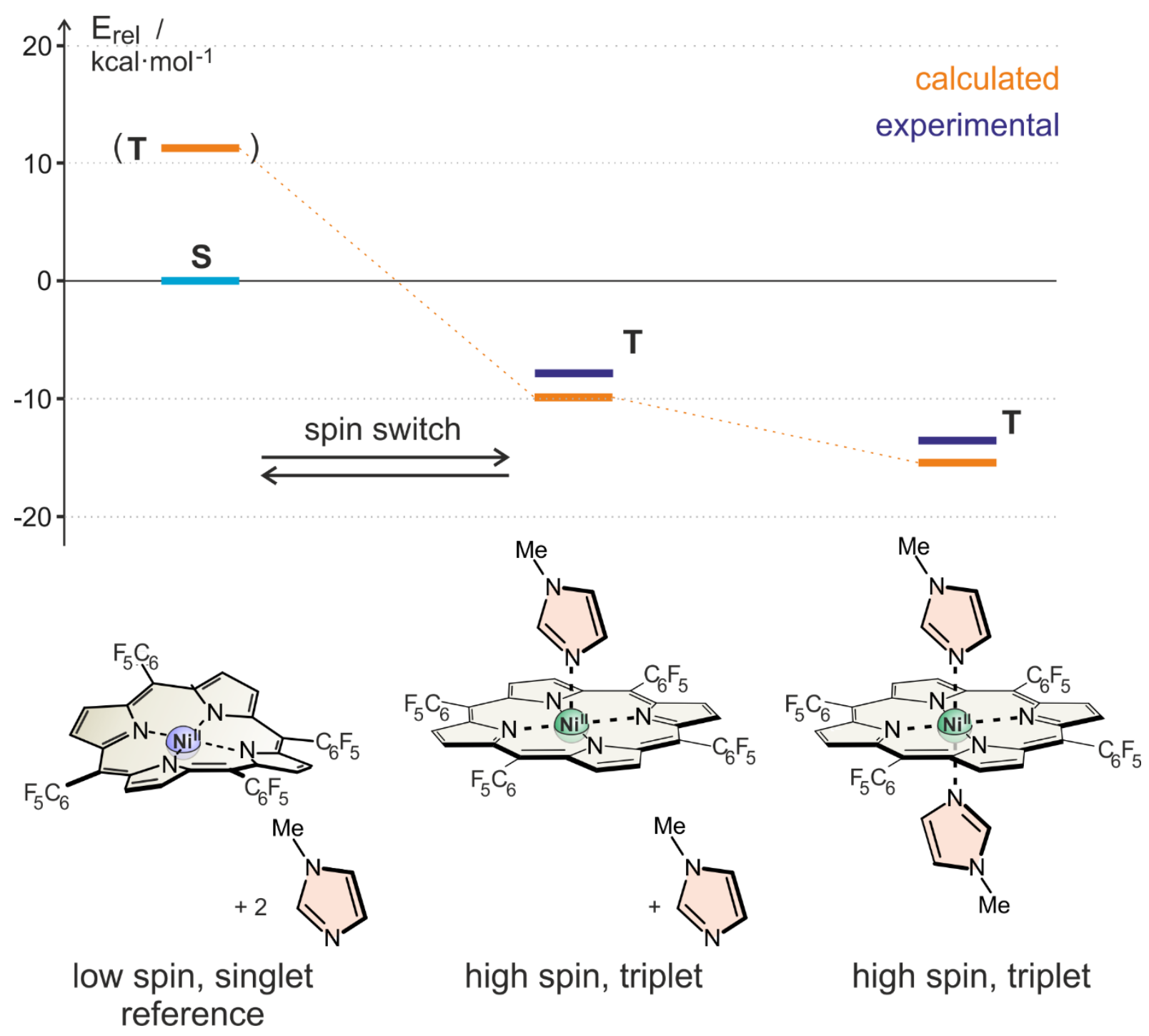

Figure S4. Calculated (B3LYP/def2TZVP//PBE/SVP) complex formation energies ( $\mathrm{kcal} \mathrm{mol}^{-1}$ ) of 1-methyl imidazole to NiTPPF 20 (orange). The energies are relative to the corresponding porphyrin in its low-spin singlet state including two unbound imidazole molecules. The experimental derived association energies for the NiTPPF 20 for the 1:1 and 1:2 complexes with imidazole are given as blue bars. Note that the experimental and calculated association energies agree quite well. 


\section{I.4 Effect of Substituents at the Phenyl Ring on the Complex Formation Energies}

The geometry optimizations of the NiTPPF 20 complexes were performed at the PBE/SVP level of density functional theory. Single point energies at the B3LYP/def2TZVP level of theory were performed on the optimized geometries. The complex formation energies $\left(\Delta E_{f}\right)$ are compared to the corresponding experimentally determined ( $\Delta H_{\text {expt1.) }}$ ) values (see section II.5).

Table S4. Calculated (B3LYP/def2TZVP//PBE/SVP) complex formation energies $\left(\Delta E_{f}\right)$ for the formation of the 5-coordinate complex of NiTPPF 20 with the investigated azoimidazole compounds $\mathbf{2}, \mathbf{2} \mathbf{b}, \mathbf{2} \mathbf{d}$ and $\mathbf{2 e}$ relative to the most stable conformer of the regarding isomer.

\begin{tabular}{|c|c|c|c|c|c|c|}
\hline$\#$ & Isomer & $\begin{array}{c}\text { Eabs } \\
\text { ligand } \\
\text { [a.u.] }\end{array}$ & $\begin{array}{c}\text { Eabs } \\
\text { NiTPPF (singlet) } \\
\text { [a.u.] } \\
\end{array}$ & $\begin{array}{c}E_{\text {abs }} \\
\text { NiTPPF }_{20} \cdot 1 \text { ligand } \\
\text { (triplet) [a.u.] }\end{array}$ & $\begin{array}{c}\Delta E_{\mathrm{f}} \\
1 \text { ligand } \\
{[\mathrm{kcal} / \mathrm{mol}]}\end{array}$ & $\begin{array}{c}\Delta H_{\text {exptl. }} \\
1 \text { ligand } \\
{[\mathrm{kcal} / \mathrm{mol}]}\end{array}$ \\
\hline \multirow[t]{4}{*}{2} & $\operatorname{cis}-\alpha$ & -605.88279113 & -5405.27432658 & -6011.16545150 & -5.23 & \multirow{2}{*}{$\begin{array}{c}-4.67 \\
( \pm 0.11)\end{array}$} \\
\hline & $c i s-\beta$ & -605.87234860 & -5405.27432658 & -6011.15820404 & -0.68 & \\
\hline & trans- $\alpha$ & -605.90308634 & -5405.27432658 & -6011.18974554 & -7.73 & \multirow{2}{*}{$\begin{array}{c}-6.84 \\
( \pm 0.20)\end{array}$} \\
\hline & trans $-\beta$ & -605.90310315 & -5405.27432658 & -6011.19035464 & -8.11 & \\
\hline \multirow[t]{4}{*}{$2 b$} & $c i s-\alpha$ & -720.39117482 & -5405.27432658 & -6125.67575539 & -6.43 & \multirow{2}{*}{$\begin{array}{c}-4.97 \\
( \pm 0.16)\end{array}$} \\
\hline & cis- $\beta$ & -720.38296164 & -5405.27432658 & -6125.67030373 & -3.01 & \\
\hline & trans- $\alpha$ & -720.41471798 & -5405.27432658 & -6125.70161616 & -7.89 & \multirow{2}{*}{$\begin{array}{c}-6.63 \\
( \pm 0.26)\end{array}$} \\
\hline & trans $-\beta$ & -720.41437364 & -5405.27432658 & -6125.70209407 & -8.19 & \\
\hline \multirow[t]{4}{*}{$2 d$} & $c i s-\alpha$ & -798.98877206 & -5405.27432658 & -6204.27420688 & -6.97 & \multirow{4}{*}{$\begin{array}{c}\text { no } \\
\text { exp. } \\
\text { value }\end{array}$} \\
\hline & cis- $\beta$ & -798.98002447 & -5405.27432658 & -6204.26644469 & -2.10 & \\
\hline & trans- $\alpha$ & -799.01086793 & -5405.27432658 & -6204.29664414 & -7.18 & \\
\hline & trans $-\beta$ & -799.01080266 & -5405.27432658 & -6204.29871214 & -8.48 & \\
\hline \multirow[t]{4}{*}{$2 e$} & $c i s-\alpha$ & -1034.74897618 & -5405.27432658 & -6440.03014528 & -4.29 & \multirow{2}{*}{$\begin{array}{c}-3.01 \\
( \pm 0.11)\end{array}$} \\
\hline & cis- $\beta$ & -1034.73891633 & -5405.27432658 & -6440.02461737 & -0.82 & \\
\hline & trans- $\alpha$ & -1034.77084972 & -5405.27432658 & -6440.05829011 & -8.23 & \multirow{2}{*}{$\begin{array}{c}-7.10 \\
( \pm 0.15)\end{array}$} \\
\hline & trans- $\beta$ & -1034.77059313 & -5405.27432658 & -6440.05866446 & -8.46 & \\
\hline
\end{tabular}

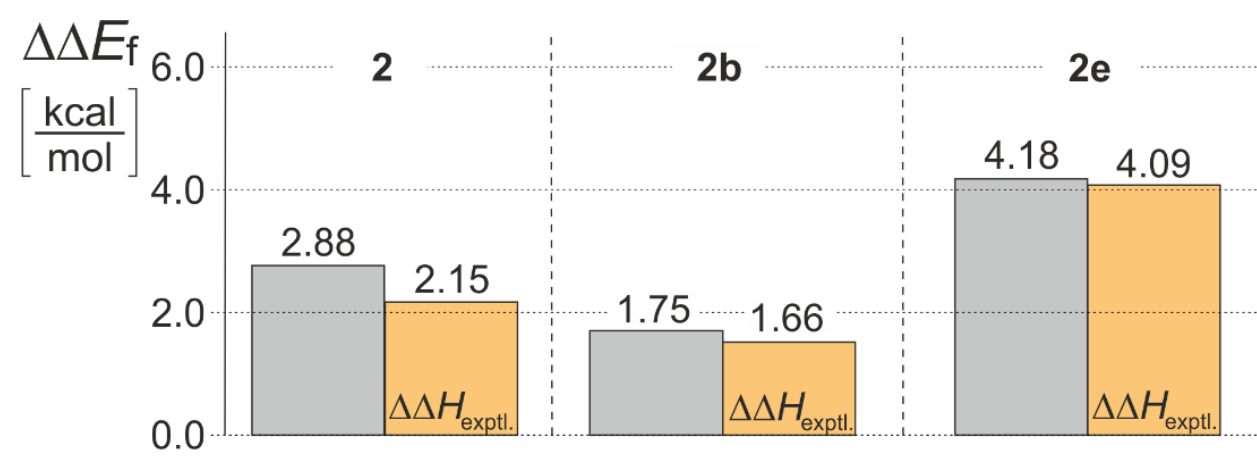

Figure S5. Comparison of calculated $\left(\Delta \Delta E_{f}, \quad B 3 L Y P / d e f 2 T Z V P / / P B E / S V P\right.$, grey columns) and experimentally derived ( $\Delta \Delta H_{\text {exptl.) }}$ ) energy differences (orange columns) of $\mathbf{2}, \mathbf{2} \mathbf{b}$ and $\mathbf{2} \mathbf{e}$ in kcal mol $\mathbf{~ m o ~}^{-1}$.

The calculated $\left(\Delta E_{\mathrm{f}}, \mathrm{B} 3 \mathrm{LYP} / \mathrm{def} 2 \mathrm{TZVP} / / \mathrm{PBE} / \mathrm{SVP}\right)$ and the experimentally determined association enthalpies ( $\Delta H_{\text {expt.) }}$ ) as well as the energy differences of the most stable complexes ( $\Delta \Delta E_{f}$ and $\Delta \Delta H_{\text {exptl., }}$ Figure S05) are in good agreement. Note that a large $\Delta \Delta E_{\mathrm{f}}(\Delta \Delta H)$ is a prerequisite for an efficient PDL. 


\section{I.5 XYZ Coordinates of the PBE/SVP optimized Ligands}

\section{I.5.1 Phenylazoimidazole (1)}

$\begin{array}{lrrr}\text { cis- } \alpha & & \\ \text { EPBE/SVP }= & -565.6163604558 \text { Hartree } & \\ \text { NImag = } & & \\ \text { C } & 1.004710 & -0.000299 & 0.936070 \\ \mathrm{C} & 1.558280 & -1.178440 & 0.391220 \\ \mathrm{C} & 2.481950 & -1.092230 & -0.661010 \\ \mathrm{C} & 2.869580 & 0.159440 & -1.169190 \\ \mathrm{C} & 2.331520 & 1.331420 & -0.610230 \\ \mathrm{C} & 1.405210 & 1.259030 & 0.440460 \\ \mathrm{~N} & 0.136890 & -0.079320 & 2.063720 \\ \mathrm{~N} & -1.120540 & -0.114940 & 1.942250 \\ \mathrm{C} & -1.795870 & -0.095720 & 0.727630 \\ \mathrm{C} & -1.566360 & -0.054450 & -0.668150 \\ \mathrm{~N} & -3.185400 & -0.139670 & 0.834490 \\ \mathrm{~N} & -2.745040 & -0.072300 & -1.348670 \\ \mathrm{C} & -3.700950 & -0.123150 & -0.418230 \\ \mathrm{H} & 1.256850 & -2.155070 & 0.802160 \\ \mathrm{H} & 2.906440 & -2.016850 & -1.086450 \\ \mathrm{H} & 3.598130 & 0.222160 & -1.993540 \\ \mathrm{H} & 2.637810 & 2.318320 & -0.995340 \\ \mathrm{H} & 0.985040 & 2.173450 & 0.888850 \\ \mathrm{H} & -0.603820 & -0.013860 & -1.191840 \\ \mathrm{H} & -4.781990 & -0.148960 & -0.618540 \\ \mathrm{H} & -3.672450 & -0.178560 & 1.734340\end{array}$

\begin{tabular}{lrrr} 
cis $-\boldsymbol{\beta}$ & \multicolumn{3}{l}{} \\
EpBE/SVP $=$ & -565.6165672927 Hartree & \\
NImag $=0$ & & \\
$\mathrm{C}$ & 0.678780 & -0.011640 & 1.152770 \\
$\mathrm{C}$ & 0.009740 & 1.164110 & 1.564640 \\
$\mathrm{C}$ & -1.266020 & 1.078500 & 2.142700 \\
$\mathrm{C}$ & -1.885200 & -0.170160 & 2.328420 \\
$\mathrm{C}$ & -1.213690 & -1.340330 & 1.932970 \\
$\mathrm{C}$ & 0.060860 & -1.269090 & 1.349910 \\
$\mathrm{~N}$ & 2.017270 & 0.061250 & 0.673360 \\
$\mathrm{~N}$ & 2.323210 & 0.100060 & -0.554050 \\
$\mathrm{C}$ & 1.431770 & 0.107080 & -1.606970 \\
$\mathrm{C}$ & 1.806680 & 0.153490 & -2.961820 \\
$\mathrm{~N}$ & 0.027600 & 0.089480 & -1.646080 \\
$\mathrm{~N}$ & 0.720530 & 0.162360 & -3.778450 \\
$\mathrm{C}$ & -0.329870 & 0.123800 & -2.957740 \\
$\mathrm{H}$ & 0.506480 & 2.138200 & 1.429630 \\
$\mathrm{H}$ & -1.780680 & 2.001960 & 2.456480 \\
$\mathrm{H}$ & -2.883730 & -0.231530 & 2.789940 \\
$\mathrm{H}$ & -1.685640 & -2.325660 & 2.083000 \\
$\mathrm{H}$ & 0.597000 & -2.183520 & 1.049010 \\
$\mathrm{H}$ & 2.837940 & 0.180990 & -3.339590 \\
$\mathrm{H}$ & -1.382900 & 0.119790 & -3.277530 \\
$\mathrm{H}$ & -0.590130 & 0.050870 & -0.830580
\end{tabular}

\section{trans- $\alpha$}

$E_{P B E / S V P}=-565.6355981556$ Hartree $\mathrm{NImag}=0$

$\begin{array}{lrrr}\text { C } & -1.023780 & -0.581900 & 0.000083 \\ \text { C } & -2.428120 & -0.419820 & 0.000070 \\ \text { C } & -3.270890 & -1.539100 & -0.000014 \\ \text { C } & -2.719710 & -2.831790 & -0.000081 \\ \text { C } & -1.319870 & -2.999840 & -0.000028 \\ \text { C } & -0.473240 & -1.887940 & 0.000062 \\ \text { N } & -0.272080 & 0.610390 & 0.000077 \\ \text { N } & 0.994620 & 0.449340 & 0.000078 \\ \text { C } & 1.728080 & 1.608950 & 0.000023 \\ \text { C } & 1.469210 & 2.993270 & -0.000049 \\ \text { N } & 3.115930 & 1.518220 & 0.000019 \\ \text { N } & 2.633660 & 3.699610 & -0.000095 \\ \text { C } & 3.607620 & 2.787380 & -0.000052 \\ \text { H } & -2.825630 & 0.607630 & 0.000131 \\ \text { H } & -4.365060 & -1.404150 & -0.000024 \\ \text { H } & -3.380320 & -3.714410 & -0.000181 \\ \text { H } & -0.890670 & -4.015990 & -0.000059 \\ \text { H } & 0.622130 & -1.993910 & 0.000123 \\ \text { H } & 0.482930 & 3.474680 & -0.000074 \\ \text { H } & 4.685390 & 3.005700 & -0.000070 \\ \text { H } & 3.629800 & 0.633680 & 0.000061\end{array}$

trans- $\beta$

$E_{P B E / S V P}=-565.6399180617$ Hartree NImag $=0$

$\begin{array}{lrrr}\text { C } & -0.249930 & -1.071480 & -0.000141 \\ \text { C } & 0.521720 & -2.255720 & -0.000301 \\ \text { C } & -0.104240 & -3.509080 & -0.000264 \\ \text { C } & -1.507050 & -3.591760 & 0.000104 \\ \text { C } & -2.281280 & -2.413680 & 0.000308 \\ \text { C } & -1.664100 & -1.159750 & 0.000200 \\ \text { N } & 0.473900 & 0.137430 & -0.000307 \\ \text { N } & -0.238140 & 1.201440 & -0.000448 \\ \text { C } & 0.494170 & 2.358620 & -0.000225 \\ \text { C } & 0.068670 & 3.697620 & -0.000460 \\ \text { N } & 1.882360 & 2.430880 & 0.000383 \\ \text { N } & 1.139000 & 4.542500 & -0.000249 \\ \text { C } & 2.212650 & 3.751430 & 0.000271 \\ \text { H } & 1.618990 & -2.156690 & -0.000352 \\ \text { H } & 0.504020 & -4.428410 & -0.000563 \\ \text { H } & -2.002690 & -4.576620 & 0.000238 \\ \text { H } & -3.382100 & -2.482310 & 0.000551 \\ \text { H } & -2.246070 & -0.225840 & 0.000495 \\ \text { H } & -0.969600 & 4.056160 & -0.000699 \\ \text { H } & 3.255990 & 4.100730 & 0.000569 \\ \text { H } & 2.473730 & 1.594530 & 0.000888\end{array}$


I.5.2 1-Methyl-phenylazoimidazole (2)

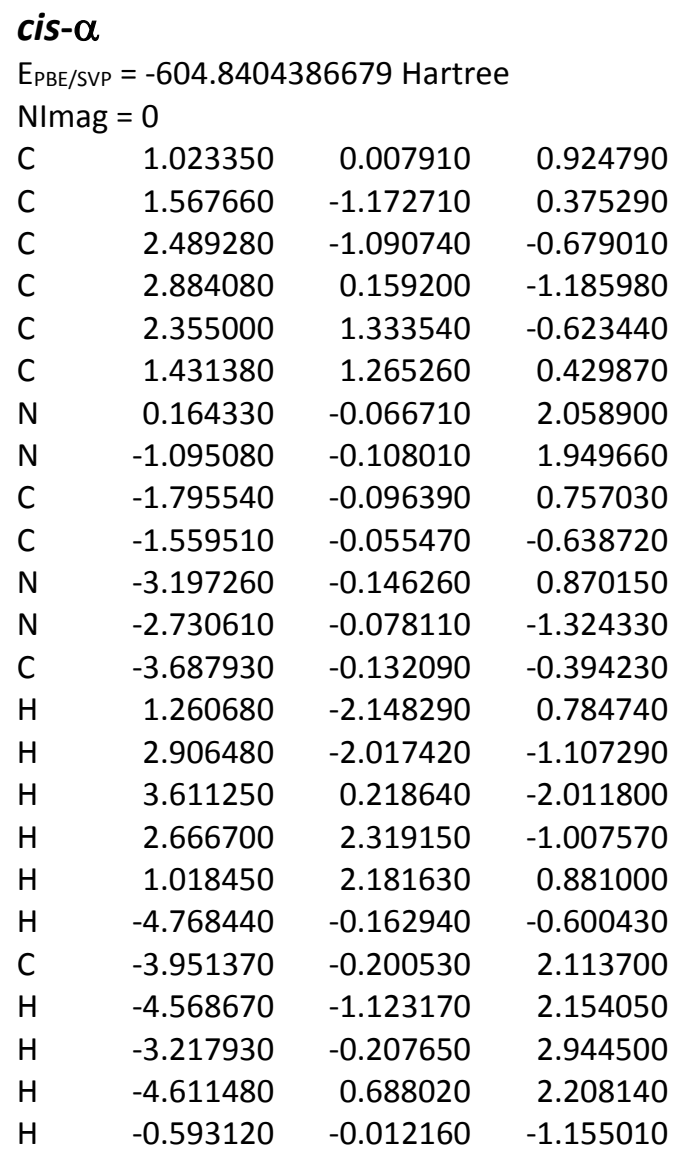

\section{cis- $\beta$}

$E_{P B E} / \mathrm{SVP}=-604.8317963959$ Hartree

NImag $=0$

$\begin{array}{lrrr}\text { C } & 0.523940 & 0.423140 & 1.207770 \\ \text { C } & -0.176360 & 1.229490 & 2.137280 \\ \text { C } & -1.212380 & 0.686110 & 2.905690 \\ \text { C } & -1.531880 & -0.680640 & 2.800200 \\ \text { C } & -0.805650 & -1.497820 & 1.914830 \\ \text { C } & 0.207360 & -0.956150 & 1.112050 \\ \text { N } & 1.644740 & 0.996130 & 0.564120 \\ \text { N } & 2.019520 & 0.728730 & -0.616230 \\ \mathrm{C} & 1.287650 & 0.102650 & -1.608740 \\ \mathrm{C} & 1.853680 & -0.658260 & -2.649160 \\ \mathrm{~N} & -0.088500 & 0.142030 & -1.906640 \\ \mathrm{~N} & 0.899600 & -1.105490 & -3.508650 \\ \mathrm{C} & -0.237220 & -0.586320 & -3.058360 \\ \mathrm{H} & 0.115550 & 2.287510 & 2.234070 \\ \mathrm{H} & -1.765060 & 1.328750 & 3.611000 \\ \mathrm{H} & -2.332760 & -1.112950 & 3.421700 \\ \mathrm{H} & -1.031110 & -2.575400 & 1.849250 \\ \mathrm{H} & 0.780040 & -1.603100 & 0.428520 \\ \mathrm{H} & 2.917850 & -0.911160 & -2.751910 \\ \mathrm{C} & -1.213220 & -0.657680 & -3.563250 \\ \mathrm{H} & -1.132660 & 0.951740 & -1.297490 \\ \mathrm{H} & -1.576310 & 0.450110 & -0.411970 \\ \mathrm{H} & -0.716320 & 1.928360 & -0.977330 \\ \mathrm{H} & -1.923320 & 1.135630 & -2.054260\end{array}$

\section{trans- $\alpha$}

$E_{P B E / S V P}=-604.8595102651$ Hartree NImag $=0$

$\begin{array}{lrrr}\mathrm{C} & -1.03014 & -0.58508 & 3.71 \mathrm{E}-04 \\ \mathrm{C} & -2.43398 & -0.41662 & 0.00334 \\ \mathrm{C} & -3.28278 & -1.53128 & 0.00298 \\ \mathrm{C} & -2.7389 & -2.82707 & -5.03 \mathrm{E}-04 \\ \mathrm{C} & -1.33997 & -3.00201 & -0.0036 \\ \mathrm{C} & -0.48737 & -1.89452 & -0.00312 \\ \mathrm{~N} & -0.27424 & 0.60411 & 4.81 \mathrm{E}-04 \\ \mathrm{~N} & 0.99316 & 0.44013 & 4.09 \mathrm{E}-04 \\ \mathrm{C} & 1.72999 & 1.59555 & -7.00 \mathrm{E}-04 \\ \mathrm{C} & 1.45924 & 2.97879 & -0.00364 \\ \mathrm{~N} & 3.12403 & 1.50213 & 9.29 \mathrm{E}-04 \\ \mathrm{~N} & 2.61566 & 3.6925 & -0.00357 \\ \mathrm{C} & 3.59223 & 2.78246 & -7.87 \mathrm{E}-04 \\ \mathrm{H} & -2.82609 & 0.6129 & 0.00562 \\ \mathrm{H} & -4.37622 & -1.3902 & 0.00539 \\ \mathrm{H} & -3.40416 & -3.70622 & -6.71 \mathrm{E}-04 \\ \mathrm{H} & -0.91593 & -4.02039 & -0.00673 \\ \mathrm{H} & 0.60727 & -2.00807 & -0.00561 \\ \mathrm{H} & 4.6699 & 3.00539 & -1.33 \mathrm{E}-04 \\ \mathrm{C} & 3.89695 & 0.27268 & 0.00349 \\ \mathrm{H} & 3.66355 & -0.33325 & 0.90396 \\ \mathrm{H} & 3.66474 & -0.33616 & -0.89531 \\ \mathrm{H} & 4.97504 & 0.53217 & 0.00379 \\ \mathrm{H} & 0.46783 & 3.45006 & -0.00598\end{array}$

\section{trans- $\beta$}

$E_{P B E / S V P}=-604.8598932150$ Hartree NImag $=0$

$\begin{array}{lrrr}\text { C } & -0.653950 & -1.269240 & 0.000000 \\ \mathrm{C} & 0.050690 & -2.494520 & 0.000000 \\ \mathrm{C} & -0.642960 & -3.711980 & 0.000000 \\ \mathrm{C} & -2.048130 & -3.718720 & 0.000000 \\ \mathrm{C} & -2.756060 & -2.499900 & 0.000000 \\ \mathrm{C} & -2.070660 & -1.281580 & 0.000000 \\ \mathrm{~N} & 0.137180 & -0.099780 & 0.000000 \\ \mathrm{~N} & -0.526440 & 0.994130 & 0.000000 \\ \mathrm{C} & 0.184930 & 2.158830 & 0.000000 \\ \mathrm{C} & -0.364860 & 3.455040 & 0.000000 \\ \mathrm{~N} & 1.577740 & 2.362490 & 0.000000 \\ \mathrm{~N} & 0.607400 & 4.402910 & 0.000000 \\ \mathrm{C} & 1.749620 & 3.717120 & 0.000000 \\ \mathrm{H} & 1.151970 & -2.458660 & 0.000000 \\ \mathrm{H} & -0.085240 & -4.662990 & 0.000000 \\ \mathrm{H} & -2.596650 & -4.675120 & 0.000000 \\ \mathrm{H} & -3.859040 & -2.507470 & 0.000000 \\ \mathrm{H} & -2.600660 & -0.317120 & 0.000000 \\ \mathrm{H} & -1.436620 & 3.697370 & 0.000000 \\ \mathrm{C} & 2.756570 & 4.163990 & 0.000000 \\ \mathrm{H} & 2.647420 & 1.376070 & 0.000000 \\ \mathrm{H} & 2.580810 & 0.724880 & 0.894220 \\ \mathrm{H} & 2.580810 & 0.724880 & -0.894220 \\ \mathrm{H} & 3.616130 & 1.919400 & 0.000000\end{array}$


I.5.3 1-iso-propyl-phenylazoimidazole (3)

\begin{tabular}{|c|c|c|c|c|c|c|c|}
\hline & & & & & & & \\
\hline & $=-683.2935$ & 32972 Hartr & & & $=-683.2823$ & 42773 Hartr & \\
\hline & $=0$ & & & & $=0$ & & \\
\hline C & 1.026700 & 0.012480 & 0.914580 & C & 0.526860 & 0.527450 & 1.402520 \\
\hline$C$ & 1.567400 & -1.168890 & 0.363000 & C & -0.282140 & 1.166030 & 2.374640 \\
\hline C & 2.490790 & -1.088230 & -0.689800 & C & -1.024560 & 0.407410 & 3.287320 \\
\hline C & 2.890950 & 0.161260 & -1.193730 & C & -0.932410 & -0.996800 & 3.281380 \\
\hline$C$ & 2.365630 & 1.336360 & -0.629200 & C & -0.091090 & -1.634520 & 2.351280 \\
\hline $\mathrm{C}$ & 1.440600 & 1.269210 & 0.422960 & C & 0.626820 & -0.887880 & 1.407840 \\
\hline $\mathrm{N}$ & 0.166370 & -0.061070 & 2.047490 & $\mathrm{~N}$ & 1.348890 & 1.355070 & 0.603750 \\
\hline$N$ & -1.092950 & -0.105520 & 1.937300 & $\mathrm{~N}$ & 1.680330 & 1.112360 & -0.595120 \\
\hline C & -1.794090 & -0.096380 & 0.745470 & C & 1.117740 & 0.196410 & -1.468130 \\
\hline C & -1.552930 & -0.053400 & -0.649610 & C & 1.855260 & -0.463930 & -2.467510 \\
\hline$N$ & -3.196500 & -0.150490 & 0.856010 & $\mathrm{~N}$ & -0.211400 & -0.243620 & -1.650100 \\
\hline $\mathrm{N}$ & -2.721240 & -0.078920 & -1.338850 & $\mathrm{~N}$ & 1.065600 & -1.299500 & -3.192490 \\
\hline C & -3.681870 & -0.136270 & -0.410670 & C & -0.159580 & -1.116110 & -2.709200 \\
\hline $\mathrm{H}$ & 1.256060 & -2.144190 & 0.769780 & $\mathrm{H}$ & -0.307560 & 2.267390 & 2.387550 \\
\hline $\mathrm{H}$ & 2.905060 & -2.015580 & -1.119490 & $\mathrm{H}$ & -1.666350 & 0.915700 & 4.025920 \\
\hline $\mathrm{H}$ & 3.619140 & 0.219700 & -2.018760 & $\mathrm{H}$ & -1.500110 & -1.592990 & 4.014130 \\
\hline $\mathrm{H}$ & 2.681440 & 2.321650 & -1.010910 & $\mathrm{H}$ & 0.008380 & -2.732830 & 2.361960 \\
\hline $\mathrm{H}$ & 1.030840 & 2.186140 & 0.875880 & $\mathrm{H}$ & 1.291720 & -1.393320 & 0.690120 \\
\hline $\mathrm{H}$ & -4.759280 & -0.169150 & -0.628830 & $\mathrm{H}$ & 2.939850 & -0.379970 & -2.622700 \\
\hline C & -3.962420 & -0.206040 & 2.116740 & C & -1.065300 & -1.573400 & -3.128950 \\
\hline $\mathrm{H}$ & -3.175760 & -0.211420 & 2.900190 & $\mathrm{H}$ & -1.441140 & 0.369740 & -1.097000 \\
\hline $\mathrm{H}$ & -0.585110 & -0.006520 & -1.163110 & $\mathrm{H}$ & -1.308590 & 0.400880 & 0.003060 \\
\hline$C$ & -4.769030 & -1.507510 & 2.208580 & C & -2.677280 & -0.486900 & -1.384560 \\
\hline $\mathrm{H}$ & -5.262650 & -1.575030 & 3.202100 & $\mathrm{H}$ & -2.536180 & -1.535290 & -1.044960 \\
\hline $\mathrm{H}$ & -5.564360 & -1.557190 & 1.431250 & $\mathrm{H}$ & -3.540350 & -0.062960 & -0.828820 \\
\hline $\mathrm{H}$ & -4.109260 & -2.393470 & 2.090070 & $\mathrm{H}$ & -2.947220 & -0.491310 & -2.463290 \\
\hline C & -4.826590 & 1.050400 & 2.284920 & C & -1.597460 & 1.807880 & -1.612910 \\
\hline $\mathrm{H}$ & -5.622140 & 1.111260 & 1.508600 & $\mathrm{H}$ & -2.490610 & 2.281000 & -1.149880 \\
\hline $\mathrm{H}$ & -5.323390 & 1.036110 & 3.279140 & $\mathrm{H}$ & -0.711580 & 2.428130 & -1.359120 \\
\hline $\mathrm{H}$ & -4.206780 & 1.970410 & 2.222480 & $\mathrm{H}$ & -1.728240 & 1.818790 & -2.717910 \\
\hline
\end{tabular}


trans- $\alpha$

$E_{P B E / S V P}=-683.3123578560$ Hartree

$\mathrm{NImag}=0$

$\begin{array}{lrrr}\text { C } & -1.030710 & -0.584580 & -0.001420 \\ \mathrm{C} & -2.433670 & -0.406630 & -0.006270 \\ \mathrm{C} & -3.290930 & -1.514640 & -0.004980 \\ \mathrm{C} & -2.757010 & -2.814540 & 0.001390 \\ \mathrm{C} & -1.359560 & -2.999430 & 0.006300 \\ \mathrm{C} & -0.498390 & -1.898320 & 0.004810 \\ \mathrm{~N} & -0.270010 & 0.601310 & -0.003110 \\ \mathrm{~N} & 0.997300 & 0.437660 & 0.000358 \\ \mathrm{C} & 1.735140 & 1.591600 & 0.000149 \\ \mathrm{C} & 1.445560 & 2.970610 & -0.000940 \\ \mathrm{~N} & 3.133830 & 1.515040 & 0.002270 \\ \mathrm{~N} & 2.591020 & 3.699620 & 0.000331 \\ \mathrm{C} & 3.581810 & 2.802230 & 0.001950 \\ \mathrm{H} & -2.818080 & 0.625770 & -0.010970 \\ \mathrm{H} & -4.383240 & -1.365090 & -0.008990 \\ \mathrm{H} & -3.428690 & -3.688760 & 0.002560 \\ \mathrm{H} & -0.942840 & -4.020770 & 0.011450 \\ \mathrm{H} & 0.594900 & -2.024490 & 0.008400 \\ \mathrm{H} & 4.652800 & 3.051310 & 0.002640 \\ \mathrm{C} & 3.932960 & 0.276450 & 0.002230 \\ \mathrm{H} & 3.170850 & -0.531540 & 0.006960 \\ \mathrm{H} & 0.447200 & 3.427020 & -0.002280 \\ \mathrm{C} & 4.777580 & 0.169280 & 1.278780 \\ \mathrm{H} & 5.302660 & -0.810140 & 1.304640 \\ \mathrm{H} & 5.549710 & 0.969410 & 1.328890 \\ \mathrm{H} & 4.139150 & 0.244550 & 2.184940 \\ \mathrm{C} & 4.767630 & 0.163410 & -1.280460 \\ \mathrm{H} & 5.538910 & 0.963680 & -1.340060 \\ \mathrm{H} & 5.292670 & -0.816050 & -1.306200 \\ \mathrm{H} & 4.122040 & 0.234610 & -2.181860\end{array}$

trans- $\beta$

$E_{P B E / S V P}=-683.3110071957$ Hartree NImag $=0$

$\begin{array}{lrrr}\text { C } & -0.372390 & -1.268340 & 0.000024 \\ \mathrm{C} & 0.275370 & -2.524420 & 0.000338 \\ \mathrm{C} & -0.473870 & -3.708630 & -0.002000 \\ \mathrm{C} & -1.877820 & -3.651560 & -0.004880 \\ \mathrm{C} & -2.528750 & -2.401520 & -0.005170 \\ \mathrm{C} & -1.788060 & -1.215990 & -0.002610 \\ \mathrm{~N} & 0.468870 & -0.134040 & 0.001800 \\ \mathrm{~N} & -0.145510 & 0.987130 & 0.001840 \\ \mathrm{C} & 0.581080 & 2.144610 & 0.002480 \\ \mathrm{C} & 0.003810 & 3.430150 & -0.000398 \\ \mathrm{~N} & 1.970000 & 2.379310 & 0.004490 \\ \mathrm{~N} & 0.952130 & 4.400340 & -0.000815 \\ \mathrm{C} & 2.110560 & 3.738050 & 0.002210 \\ \mathrm{H} & 1.377140 & -2.542430 & 0.002230 \\ \mathrm{H} & 0.040020 & -4.684080 & -0.001690 \\ \mathrm{H} & -2.469330 & -4.581900 & -0.006990 \\ \mathrm{H} & -3.630920 & -2.357860 & -0.007600 \\ \mathrm{H} & -2.273330 & -0.228320 & -0.002810 \\ \mathrm{H} & -1.073730 & 3.646330 & -0.002410 \\ \mathrm{C} & 3.100860 & 4.218230 & 0.003330 \\ \mathrm{H} & 3.082920 & 1.398370 & 0.007510 \\ \mathrm{H} & 2.574330 & 0.415080 & 0.018320 \\ \mathrm{C} & 3.925160 & 1.540190 & 1.281810 \\ \mathrm{H} & 4.698540 & 0.741830 & 1.312660 \\ \mathrm{H} & 4.449330 & 2.520860 & 1.328070 \\ \mathrm{H} & 3.292200 & 1.440100 & 2.189630 \\ \mathrm{C} & 3.911350 & 1.517550 & -1.278090 \\ \mathrm{H} & 4.431460 & 2.498950 & -1.348620 \\ \mathrm{H} & 4.686960 & 0.721070 & -1.301810 \\ \mathrm{H} & 3.269000 & 1.398030 & -2.176870\end{array}$


I.5.4 1-Trimethylsilyl-phenylazoimidazole (4)

\section{cis- $\alpha$}

$E_{P B E / S V P}=-973.7934459327$ Hartree

NImag $=0$

$\begin{array}{lrrr}\mathrm{C} & -3.513620 & 2.007580 & -2.066790 \\ \mathrm{C} & -2.186160 & 1.556630 & -2.034840 \\ \mathrm{C} & -1.291770 & 2.073450 & -1.072350 \\ \mathrm{C} & -1.733470 & 3.058550 & -0.163640 \\ \mathrm{C} & -3.065630 & 3.496470 & -0.201500 \\ \mathrm{C} & -3.962010 & 2.973050 & -1.148860 \\ \mathrm{H} & -4.206680 & 1.598610 & -2.820890 \\ \mathrm{H} & -1.827620 & 0.804050 & -2.755250 \\ \mathrm{H} & -1.021280 & 3.472880 & 0.567710 \\ \mathrm{H} & -3.404910 & 4.261150 & 0.517090 \\ \mathrm{H} & -5.005970 & 3.324530 & -1.178550 \\ \mathrm{~N} & 0.082750 & 1.700810 & -1.100310 \\ \mathrm{~N} & 0.512510 & 0.666670 & -0.511190 \\ \mathrm{C} & -1.584800 & -0.357750 & 0.703570 \\ \mathrm{C} & -0.463640 & -1.994580 & 1.508920 \\ \mathrm{H} & -0.200960 & -2.912660 & 2.053950 \\ \mathrm{~N} & -1.691550 & -1.467910 & 1.479840 \\ \mathrm{~N} & 0.454750 & -1.287780 & 0.793020 \\ \mathrm{C} & -0.254350 & -0.203570 & 0.248810 \\ \mathrm{H} & -2.449160 & 0.285930 & 0.500000 \\ \mathrm{C} & 2.584280 & -1.873860 & -1.277640 \\ \mathrm{H} & 2.384800 & -0.938000 & -1.841550 \\ \mathrm{H} & 3.646950 & -2.164600 & -1.439510 \\ \mathrm{H} & 1.941710 & -2.680820 & -1.694330 \\ \mathrm{C} & 3.227040 & -0.192300 & 1.306780 \\ \mathrm{H} & 4.319790 & -0.397270 & 1.245270 \\ \mathrm{H} & 3.013110 & 0.749910 & 0.759170 \\ \mathrm{H} & 2.965710 & -0.045850 & 2.378170 \\ \mathrm{C} & 2.556030 & -3.218540 & 1.521800 \\ \mathrm{H} & 3.630330 & -3.497730 & 1.433360 \\ \mathrm{H} & 2.332360 & -3.108340 & 2.606060 \\ \mathrm{H} & 1.959730 & -4.069770 & 1.125070 \\ \mathrm{Si} & 2.251710 & -1.618940 & 0.558580\end{array}$

cis- $\beta$

$E_{P B E / S V P}=-973.7780588708$ Hartree NImag $=0$

$\begin{array}{lrrr}\mathrm{C} & 0.560340 & 0.884610 & 3.411330 \\ \mathrm{C} & 1.437120 & 0.481500 & 2.397740 \\ \mathrm{C} & 1.126640 & -0.628470 & 1.573190 \\ \mathrm{C} & -0.059710 & -1.363730 & 1.830910 \\ \mathrm{C} & -0.907600 & -0.979280 & 2.877700 \\ \mathrm{C} & -0.616160 & 0.153290 & 3.659600 \\ \mathrm{H} & 0.806490 & 1.762180 & 4.031820 \\ \mathrm{H} & 2.391470 & 1.005760 & 2.227480 \\ \mathrm{H} & -0.296130 & -2.256190 & 1.231320 \\ \mathrm{H} & -1.814520 & -1.571870 & 3.083740 \\ \mathrm{H} & -1.296630 & 0.454770 & 4.472460 \\ \mathrm{~N} & 2.157490 & -1.066260 & 0.701130 \\ \mathrm{~N} & 1.978930 & -1.707530 & -0.375980 \\ \mathrm{C} & 0.372250 & -3.050500 & -1.668850 \\ \mathrm{C} & -1.172650 & -1.633500 & -2.042370 \\ \mathrm{H} & 0.915680 & -4.005120 & -1.651700 \\ \mathrm{~N} & -2.065060 & -1.150460 & -2.467000 \\ \mathrm{~N} & -0.862930 & -2.909160 & -2.229060 \\ \mathrm{C} & -0.231730 & -0.911590 & -1.330390 \\ \mathrm{H} & 0.784230 & -1.852980 & -1.063800 \\ \mathrm{C} & -1.303070 & 1.591610 & 0.046220 \\ \mathrm{H} & -2.323000 & 1.151250 & -0.009840 \\ \mathrm{H} & -0.888070 & 1.374870 & 1.052480 \\ \mathrm{H} & -1.405500 & 2.695810 & -0.057440 \\ \mathrm{C} & 1.562470 & 1.530900 & -1.307260 \\ \mathrm{H} & 2.146980 & 1.083490 & -2.141210 \\ \mathrm{H} & 1.565670 & 2.636260 & -1.443890 \\ \mathrm{H} & 2.090950 & 1.302560 & -0.358990 \\ \mathrm{C} & -0.990140 & 1.419410 & -3.011800 \\ \mathrm{H} & -0.906810 & 2.522800 & -3.139630 \\ \mathrm{H} & -0.462960 & 0.938320 & -3.865000 \\ \mathrm{H} & -2.070260 & 1.163200 & -3.082870 \\ \mathrm{Si} & -0.223780 & 0.934050 & -1.350030\end{array}$


trans- $\alpha$

$E_{P B E / S V P}=-973.8115198197$ Hartree

NImag $=0$

$\begin{array}{lrrr}\mathrm{C} & -1.569040 & 2.279010 & -4.285690 \\ \mathrm{C} & -1.009420 & 2.540820 & -3.028520 \\ \mathrm{C} & -0.701750 & 1.481140 & -2.143820 \\ \mathrm{C} & -0.961780 & 0.146530 & -2.546050 \\ \mathrm{C} & -1.517790 & -0.107810 & -3.803420 \\ \mathrm{C} & -1.825010 & 0.954020 & -4.677740 \\ \mathrm{H} & -1.806930 & 3.112730 & -4.966680 \\ \mathrm{H} & -0.794460 & 3.566930 & -2.690330 \\ \mathrm{H} & -0.716080 & -0.673880 & -1.854640 \\ \mathrm{H} & -1.716650 & -1.147410 & -4.114090 \\ \mathrm{H} & -2.263270 & 0.744270 & -5.667410 \\ \mathrm{~N} & -0.142990 & 1.867020 & -0.909610 \\ \mathrm{~N} & 0.089710 & 0.913900 & -0.090990 \\ \mathrm{C} & 1.057990 & 2.513250 & 1.675790 \\ \mathrm{C} & 1.453660 & 0.990550 & 3.124890 \\ \mathrm{H} & 1.004440 & 3.506480 & 1.211460 \\ \mathrm{~N} & 1.768580 & 0.480330 & 4.046490 \\ \mathrm{~N} & 1.556220 & 2.306660 & 2.924090 \\ \mathrm{C} & 0.911240 & 0.309900 & 2.067370 \\ \mathrm{H} & 0.644690 & 1.294360 & 1.106100 \\ \mathrm{C} & -1.258760 & -1.742170 & 1.655430 \\ \mathrm{H} & -1.497810 & -2.827470 & 1.588730 \\ \mathrm{H} & -1.838480 & -1.313860 & 2.502790 \\ \mathrm{H} & -1.597050 & -1.248110 & 0.719370 \\ \mathrm{C} & 1.157500 & -2.231820 & 3.550310 \\ \mathrm{H} & 0.989290 & -3.332340 & 3.530720 \\ \mathrm{H} & 2.241410 & -2.064920 & 3.736340 \\ \mathrm{H} & 0.586680 & -1.824880 & 4.414100 \\ \mathrm{C} & 1.629030 & -2.147070 & 0.477860 \\ \mathrm{H} & 1.344200 & -1.647090 & -0.472650 \\ \mathrm{H} & 2.712450 & -1.966190 & 0.654070 \\ \mathrm{H} & 1.478710 & -3.243880 & 0.358760 \\ \mathrm{Si} & 0.591480 & -1.488990 & 1.906970\end{array}$

trans- $\beta$

$E_{P B E / S V P}=-973.8128414290$ Hartree NImag $=0$

$\begin{array}{lrrr}\mathrm{C} & -3.880560 & -1.659700 & 0.002040 \\ \mathrm{C} & -2.540440 & -1.248460 & -0.000262 \\ \mathrm{C} & -2.214160 & 0.127640 & 0.001010 \\ \mathrm{C} & -3.256090 & 1.087890 & 0.004750 \\ \mathrm{C} & -4.589910 & 0.669470 & 0.007050 \\ \mathrm{C} & -4.909670 & -0.703290 & 0.005680 \\ \mathrm{H} & -4.124460 & -2.734830 & 0.001030 \\ \mathrm{H} & -1.720630 & -1.984920 & -0.003000 \\ \mathrm{H} & -2.982600 & 2.153600 & 0.005850 \\ \mathrm{H} & -5.398240 & 1.420060 & 0.009820 \\ \mathrm{H} & -5.964160 & -1.024550 & 0.007300 \\ \mathrm{~N} & -0.838320 & 0.449420 & -0.001120 \\ \mathrm{~N} & -0.576280 & 1.700250 & -0.001450 \\ \mathrm{C} & 1.233740 & 3.362300 & -0.004870 \\ \mathrm{C} & 2.933980 & 2.084250 & -0.006070 \\ \mathrm{H} & 0.627510 & 4.278930 & -0.004740 \\ \mathrm{~N} & 3.975200 & 1.732690 & -0.006900 \\ \mathrm{~N} & 2.591800 & 3.372510 & -0.006590 \\ \mathrm{C} & 1.876130 & 1.206640 & -0.004160 \\ \mathrm{H} & 0.741890 & 2.045540 & -0.003330 \\ \mathrm{C} & 1.359330 & -1.357740 & -1.600940 \\ \mathrm{H} & 1.847210 & -0.885100 & -2.482530 \\ \mathrm{H} & 1.580400 & -2.448890 & -1.637720 \\ \mathrm{H} & 0.263060 & -1.212760 & -1.683270 \\ \mathrm{C} & 1.359110 & -1.353090 & 1.600380 \\ \mathrm{H} & 1.595620 & -2.440550 & 1.648340 \\ \mathrm{H} & 1.834900 & -0.865740 & 2.480600 \\ \mathrm{H} & 0.260490 & -1.222750 & 1.675380 \\ \mathrm{C} & 3.921540 & -0.913110 & -0.000384 \\ \mathrm{H} & 4.421390 & -0.497620 & -0.903150 \\ \mathrm{H} & 4.420680 & -0.496370 & 0.902190 \\ \mathrm{H} & 4.107660 & -2.011250 & 0.000355 \\ \mathrm{Si} & 2.043850 & -0.630470 & -0.001300\end{array}$




\section{I.5.5 1-tert-Butyl-phenylazoimidazole (5)}

\begin{tabular}{|c|c|c|c|c|c|c|c|}
\hline & & & & cis & & & \\
\hline & $=-722.5142$ & 71297 Hartr & & & $=-722.4961$ & 53889 Hartr & \\
\hline & & & & & & & \\
\hline C & -3.313470 & 2.638080 & -1.968800 & C & 2.358180 & 1.713470 & 2.130050 \\
\hline C & -1.964100 & 2.254830 & -1.996030 & C & 2.560990 & 0.845270 & 1.050650 \\
\hline C & -1.230160 & 2.177200 & -0.792860 & C & 1.796780 & -0.340730 & 0.927550 \\
\hline C & -1.849940 & 2.527040 & 0.428180 & C & 0.871440 & -0.674570 & 1.949660 \\
\hline C & -3.193900 & 2.925130 & 0.440170 & C & 0.713850 & 0.176260 & 3.052420 \\
\hline C & -3.935090 & 2.973870 & -0.753820 & C & 1.435180 & 1.380810 & 3.138940 \\
\hline $\mathrm{H}$ & -3.883400 & 2.682330 & -2.911780 & $\mathrm{H}$ & 2.942140 & 2.645930 & 2.200110 \\
\hline $\mathrm{H}$ & -1.463450 & 2.007260 & -2.945560 & $\mathrm{H}$ & 3.317150 & 1.059310 & 0.278260 \\
\hline $\mathrm{H}$ & -1.264770 & 2.490180 & 1.361180 & $\mathrm{H}$ & 0.299080 & -1.612730 & 1.893290 \\
\hline $\mathrm{H}$ & -3.668810 & 3.200310 & 1.396690 & $\mathrm{H}$ & 0.009220 & -0.102510 & 3.853380 \\
\hline $\mathrm{H}$ & -4.992180 & 3.284370 & -0.738150 & $\mathrm{H}$ & 1.291320 & 2.051090 & 4.001820 \\
\hline $\mathrm{N}$ & 0.163350 & 1.893690 & -0.837770 & $\mathrm{~N}$ & 2.168420 & -1.218000 & -0.125540 \\
\hline $\mathrm{N}$ & 0.658610 & 0.788450 & -0.468800 & $\mathrm{~N}$ & 1.378640 & -1.995210 & -0.734300 \\
\hline C & -1.424480 & -0.732520 & -0.071560 & C & -0.735620 & -3.161090 & -0.312590 \\
\hline C & -0.288670 & -2.412820 & 0.606730 & C & -2.168960 & -1.585730 & -0.398390 \\
\hline $\mathrm{H}$ & -0.032640 & -3.410690 & 0.982700 & $\mathrm{H}$ & -0.307200 & -4.158650 & -0.145070 \\
\hline $\mathrm{N}$ & -1.536630 & -2.016530 & 0.345110 & $\mathrm{~N}$ & -3.124340 & -1.046670 & -0.444640 \\
\hline $\mathrm{N}$ & 0.647900 & -1.454100 & 0.370110 & $\mathrm{~N}$ & -2.055640 & -2.876550 & -0.123960 \\
\hline C & -0.069940 & -0.322470 & -0.076400 & C & -0.973110 & -0.971370 & -0.711950 \\
\hline $\mathrm{H}$ & -2.302600 & -0.145960 & -0.366600 & $\mathrm{H}$ & -0.014200 & -2.000760 & -0.627580 \\
\hline C & 2.132460 & -1.582240 & 0.544620 & C & -0.906860 & 0.397710 & -1.342060 \\
\hline C & 2.616620 & -0.512700 & 1.544940 & C & 0.414370 & 0.589360 & -2.106290 \\
\hline $\mathrm{H}$ & 3.711310 & -0.622460 & 1.704750 & $\mathrm{H}$ & 0.585190 & -0.230530 & -2.836280 \\
\hline $\mathrm{H}$ & 2.416290 & 0.506730 & 1.157370 & $\mathrm{H}$ & 0.355980 & 1.545150 & -2.669070 \\
\hline $\mathrm{H}$ & 2.108190 & -0.633480 & 2.526780 & $\mathrm{H}$ & 1.293010 & 0.654660 & -1.436980 \\
\hline C & 2.816560 & -1.411160 & -0.827520 & C & -1.068740 & 1.470540 & -0.248260 \\
\hline $\mathrm{H}$ & 3.914730 & -1.540670 & -0.713330 & $\mathrm{H}$ & -0.240080 & 1.428490 & 0.488460 \\
\hline $\mathrm{H}$ & 2.449110 & -2.176000 & -1.546520 & $\mathrm{H}$ & -1.074340 & 2.481370 & -0.711200 \\
\hline $\mathrm{H}$ & 2.616080 & -0.401460 & -1.240260 & $\mathrm{H}$ & -2.027490 & 1.337290 & 0.298730 \\
\hline C & 2.459750 & -2.978330 & 1.103590 & C & -2.057700 & 0.516870 & -2.369370 \\
\hline $\mathrm{H}$ & 3.559660 & -3.051780 & 1.236950 & $\mathrm{H}$ & -2.011740 & -0.306070 & -3.115310 \\
\hline $\mathrm{H}$ & 1.990100 & -3.153210 & 2.096250 & $\mathrm{H}$ & -3.060610 & 0.505140 & -1.893500 \\
\hline $\mathrm{H}$ & 2.153520 & -3.790860 & 0.409670 & $\mathrm{H}$ & -1.964290 & 1.482470 & -2.910990 \\
\hline
\end{tabular}


trans- $\alpha$

$E_{P B E / S V P}=-722.5336838549$ Hartree

$\mathrm{NImag}=0$

\begin{tabular}{lrrr}
$\mathrm{C}$ & -3.787420 & 3.389880 & -0.318900 \\
$\mathrm{C}$ & -2.415440 & 3.225880 & -0.089280 \\
$\mathrm{C}$ & -1.817900 & 1.947980 & -0.189740 \\
$\mathrm{C}$ & -2.622690 & 0.832310 & -0.533320 \\
$\mathrm{C}$ & -3.990640 & 1.004150 & -0.764800 \\
$\mathrm{C}$ & -4.579820 & 2.279930 & -0.657730 \\
$\mathrm{H}$ & -4.243560 & 4.390110 & -0.235530 \\
$\mathrm{H}$ & -1.765050 & 4.074540 & 0.175590 \\
$\mathrm{H}$ & -2.150740 & -0.158450 & -0.616540 \\
$\mathrm{H}$ & -4.613340 & 0.134860 & -1.035390 \\
$\mathrm{H}$ & -5.659340 & 2.406230 & -0.842830 \\
$\mathrm{~N}$ & -0.432680 & 1.907430 & 0.061760 \\
$\mathrm{~N}$ & 0.092460 & 0.742220 & 0.044170 \\
$\mathrm{C}$ & 2.411460 & 1.728110 & 0.472200 \\
$\mathrm{C}$ & 3.460040 & -0.132880 & 0.553950 \\
$\mathrm{H}$ & 2.224040 & 2.809550 & 0.488350 \\
$\mathrm{~N}$ & 4.265100 & -0.871200 & 0.652230 \\
$\mathrm{~N}$ & 3.642700 & 1.186970 & 0.643890 \\
$\mathrm{C}$ & 2.160060 & -0.489150 & 0.326290 \\
$\mathrm{H}$ & 1.447410 & 0.721600 & 0.269870 \\
$\mathrm{C}$ & 1.611390 & -1.873110 & 0.177670 \\
$\mathrm{C}$ & 0.559100 & -2.114340 & 1.279770 \\
$\mathrm{H}$ & 1.014730 & -2.001470 & 2.287840 \\
$\mathrm{H}$ & -0.279630 & -1.393950 & 1.185370 \\
$\mathrm{H}$ & 0.155560 & -3.146660 & 1.193520 \\
$\mathrm{C}$ & 2.749910 & -2.896440 & 0.330040 \\
$\mathrm{H}$ & 2.323960 & -3.915820 & 0.216720 \\
$\mathrm{H}$ & 3.531070 & -2.770920 & -0.450930 \\
$\mathrm{H}$ & 3.229340 & -2.842410 & 1.331380 \\
$\mathrm{C}$ & 0.986490 & -2.013420 & -1.225660 \\
$\mathrm{H}$ & 1.750290 & -1.838250 & -2.014600 \\
$\mathrm{H}$ & 0.580570 & -3.040400 & -1.354950 \\
$\mathrm{H}$ & 0.162570 & -1.282870 & -1.360410 \\
& & & \\
\hline
\end{tabular}

trans- $\beta$

$E_{P B E / S V P}=-722.5307215653$ Hartree NImag $=0$

$\begin{array}{lrrr}\text { C } & -4.177770 & -1.665900 & 0.002020 \\ \text { C } & -2.803630 & -1.390200 & 0.001480 \\ \text { C } & -2.342780 & -0.054240 & 0.000436 \\ \text { C } & -3.282180 & 1.005730 & -0.000250 \\ \text { C } & -4.651400 & 0.722860 & 0.000343 \\ \text { C } & -5.106170 & -0.611110 & 0.001510 \\ \text { H } & -4.528240 & -2.711110 & 0.002830 \\ \text { H } & -2.056820 & -2.200440 & 0.001940 \\ \text { H } & -2.903050 & 2.038590 & -0.001070 \\ \text { H } & -5.380950 & 1.550260 & -0.000236 \\ \text { H } & -6.187620 & -0.825690 & 0.001810 \\ \text { N } & -0.938420 & 0.119580 & 0.000415 \\ \text { N } & -0.556300 & 1.336540 & 0.001030 \\ \text { C } & 1.142160 & 3.041970 & 0.003310 \\ \text { C } & 2.958990 & 1.946790 & 0.002130 \\ \text { H } & 0.440260 & 3.887940 & 0.004410 \\ \text { N } & 4.029880 & 1.709850 & 0.002020 \\ \text { N } & 2.484920 & 3.193730 & 0.003840 \\ \mathrm{C} & 1.998920 & 0.971750 & 0.000611 \\ \text { H } & 0.763560 & 1.683160 & 0.001340 \\ \mathrm{C} & 2.273550 & -0.504650 & -0.001470 \\ \mathrm{C} & 1.670670 & -1.126020 & 1.273440 \\ \mathrm{H} & 1.883010 & -2.217350 & 1.291270 \\ \mathrm{H} & 2.119930 & -0.668420 & 2.182090 \\ \mathrm{H} & 0.570120 & -0.983890 & 1.290920 \\ \mathrm{C} & 3.794470 & -0.750070 & -0.002210 \\ \mathrm{H} & 4.289040 & -0.335910 & -0.907440 \\ \mathrm{H} & 4.289480 & -0.338430 & 0.903940 \\ \mathrm{H} & 3.965690 & -1.847150 & -0.003710 \\ \mathrm{C} & 1.669990 & -1.122380 & -1.277870 \\ \mathrm{H} & 1.883110 & -2.213480 & -1.299440 \\ \mathrm{H} & 0.569350 & -0.980790 & -1.294080 \\ \mathrm{H} & 2.118260 & -0.661520 & -2.185380\end{array}$


I.5.6 1-Methyl-4'-fluorophenylazoimidazole (2a)

\section{cis- $\alpha$}

$E_{P B E} / \mathrm{SVP}=-703.9186171982$ Hartree

$\mathrm{NImag}=0$

$\begin{array}{lrrr}\text { C } & 1.524870 & -0.212140 & 0.549310 \\ \mathrm{C} & 2.384140 & -1.239080 & 0.102030 \\ \mathrm{C} & 3.329840 & -0.987680 & -0.900740 \\ \mathrm{C} & 3.443900 & 0.308050 & -1.421930 \\ \mathrm{C} & 2.629430 & 1.354030 & -0.963570 \\ \mathrm{C} & 1.664140 & 1.090320 & 0.016230 \\ \mathrm{~N} & 0.654260 & -0.478590 & 1.642290 \\ \mathrm{~N} & -0.604250 & -0.374820 & 1.559040 \\ \mathrm{C} & -1.325020 & -0.159710 & 0.397620 \\ \mathrm{C} & -1.139560 & -0.182390 & -1.005860 \\ \mathrm{~N} & -2.715420 & -0.025490 & 0.559500 \\ \mathrm{~N} & -2.328330 & -0.069950 & -1.650460 \\ \mathrm{C} & -3.248070 & 0.033030 & -0.687560 \\ \mathrm{H} & 2.293740 & -2.241780 & 0.548220 \\ \mathrm{H} & 3.994890 & -1.780800 & -1.276220 \\ \mathrm{~F} & 4.364680 & 0.556270 & -2.370890 \\ \mathrm{H} & 2.762090 & 2.364620 & -1.380640 \\ \mathrm{H} & 1.016950 & 1.902060 & 0.384340 \\ \mathrm{H} & -4.328720 & 0.155760 & -0.854790 \\ \mathrm{C} & -3.416420 & 0.056490 & 1.831120 \\ \mathrm{H} & -4.375970 & -0.496100 & 1.764660 \\ \mathrm{H} & -2.765260 & -0.395010 & 2.606140 \\ \mathrm{H} & -3.619380 & 1.114540 & 2.107590 \\ \mathrm{H} & -0.196530 & -0.291630 & -1.555420\end{array}$

cis- $\beta$

$E_{P B E / S V P}=-703.9101367469$ Hartree $\mathrm{NImag}=0$

$\begin{array}{lrrr}\text { C } & 0.656190 & 0.448980 & 1.198990 \\ \mathrm{C} & -0.023750 & 1.233940 & 2.162690 \\ \mathrm{C} & -1.037730 & 0.682820 & 2.951790 \\ \mathrm{C} & -1.337110 & -0.681740 & 2.817230 \\ \mathrm{C} & -0.647150 & -1.496240 & 1.906480 \\ \mathrm{C} & 0.338170 & -0.929880 & 1.088980 \\ \mathrm{~N} & 1.760240 & 1.047890 & 0.549150 \\ \mathrm{~N} & 2.147600 & 0.770530 & -0.626100 \\ \mathrm{C} & 1.433920 & 0.105230 & -1.606540 \\ \mathrm{C} & 2.006180 & -0.686200 & -2.619760 \\ \mathrm{~N} & 0.060690 & 0.139580 & -1.915050 \\ \mathrm{~N} & 1.056110 & -1.154880 & -3.473350 \\ \mathrm{C} & -0.082510 & -0.620690 & -3.047790 \\ \mathrm{H} & 0.267560 & 2.290470 & 2.273830 \\ \mathrm{H} & -1.586810 & 1.285290 & 3.692050 \\ \mathrm{~F} & -2.291860 & -1.224580 & 3.591830 \\ \mathrm{H} & -0.889670 & -2.569230 & 1.852970 \\ \mathrm{H} & 0.890340 & -1.567550 & 0.380830 \\ \mathrm{H} & 3.069850 & -0.946280 & -2.707690 \\ \mathrm{H} & -1.054520 & -0.702210 & -3.558580 \\ \mathrm{C} & -0.980100 & 0.973930 & -1.334890 \\ \mathrm{H} & -1.444100 & 0.493940 & -0.447510 \\ \mathrm{H} & -0.553380 & 1.949500 & -1.024870 \\ \mathrm{H} & -1.758140 & 1.157360 & -2.104700\end{array}$


trans- $\alpha$

$E_{P B E / S V P}=-703.9390150324$ Hartree

NImag $=0$

$\begin{array}{lrrr}\text { C } & -1.026100 & -0.569050 & -0.000487 \\ \text { C } & -2.431440 & -0.406640 & -0.000346 \\ \text { C } & -3.282400 & -1.517250 & 0.000092 \\ \text { C } & -2.715280 & -2.799040 & 0.000599 \\ \text { C } & -1.322450 & -2.992330 & -0.000071 \\ \text { C } & -0.481500 & -1.878930 & -0.000557 \\ \text { N } & -0.271780 & 0.617700 & -0.000573 \\ \text { N } & 0.995920 & 0.452120 & -0.000067 \\ \text { C } & 1.735950 & 1.605120 & -0.000014 \\ \text { C } & 1.470240 & 2.989280 & 0.000192 \\ \text { N } & 3.129430 & 1.506260 & 0.000108 \\ \text { N } & 2.629560 & 3.698470 & 0.000352 \\ \text { C } & 3.602650 & 2.784980 & 0.000171 \\ \text { H } & -2.830000 & 0.620110 & -0.000405 \\ \text { H } & -4.378150 & -1.410250 & 0.000167 \\ \text { F } & -3.519910 & -3.874790 & 0.000855 \\ \text { H } & -0.925970 & -4.020130 & 0.000028 \\ \text { H } & 0.613120 & -1.990780 & -0.001040 \\ \text { H } & 4.681220 & 3.003290 & 0.000076 \\ \mathrm{C} & 3.896910 & 0.273480 & 0.000278 \\ \text { H } & 3.662080 & -0.332490 & 0.900420 \\ \text { H } & 3.660940 & -0.333290 & -0.899020 \\ \text { H } & 4.976170 & 0.527800 & -0.000526 \\ \text { H } & 0.480610 & 3.464350 & 0.000165\end{array}$

trans- $\beta$

$E_{P B E / S V P}=-703.9392923658$ Hartree $\mathrm{NImag}=0$

$\begin{array}{lrrr}\text { C } & -0.641910 & -1.262940 & -0.000925 \\ \text { C } & 0.055900 & -2.492770 & -0.006450 \\ \text { C } & -0.635010 & -3.709950 & -0.006070 \\ \text { C } & -2.036500 & -3.688430 & 0.000400 \\ \text { C } & -2.756840 & -2.481150 & 0.006450 \\ \text { C } & -2.059760 & -1.272180 & 0.005710 \\ \text { N } & 0.148910 & -0.096380 & -0.002070 \\ \text { N } & -0.515690 & 0.997550 & -0.003160 \\ \text { C } & 0.191580 & 2.164310 & -0.003120 \\ \text { C } & -0.363620 & 3.458200 & -0.006810 \\ \text { N } & 1.583370 & 2.373670 & 0.000643 \\ \text { N } & 0.604750 & 4.410080 & -0.005400 \\ \mathrm{C} & 1.749750 & 3.729360 & -0.000869 \\ \text { H } & 1.157110 & -2.465690 & -0.011480 \\ \text { H } & -0.108620 & -4.676920 & -0.010490 \\ \text { F } & -2.712690 & -4.849810 & 0.000991 \\ \text { H } & -3.857820 & -2.515780 & 0.011710 \\ \text { H } & -2.586910 & -0.306360 & 0.010270 \\ \text { H } & -1.436420 & 3.695770 & -0.010520 \\ \text { H } & 2.754880 & 4.180210 & 0.001710 \\ \text { C } & 2.656590 & 1.391200 & 0.006230 \\ \text { H } & 2.589070 & 0.741180 & 0.901390 \\ \text { H } & 2.596500 & 0.738890 & -0.887780 \\ \text { H } & 3.623390 & 1.937940 & 0.009630\end{array}$


I.5.7 1-Methyl-4'-methoxyphenylazoimidazole (2b)

\section{cis- $\alpha$}

$E_{P B E} / \mathrm{SVP}=-719.1478560887$ Hartree

NImag $=0$

$\begin{array}{lrrr}\text { C } & 0.664690 & -0.343780 & 0.918110 \\ \mathrm{C} & 1.690920 & -1.243560 & 0.564330 \\ \mathrm{C} & 2.711460 & -0.870100 & -0.322370 \\ \mathrm{C} & 2.754280 & 0.448060 & -0.826220 \\ \mathrm{C} & 1.758270 & 1.373320 & -0.429440 \\ \mathrm{C} & 0.723390 & 0.982340 & 0.417320 \\ \mathrm{~N} & -0.272070 & -0.756770 & 1.898230 \\ \mathrm{~N} & -1.522850 & -0.585360 & 1.789330 \\ \mathrm{C} & -2.201440 & -0.204170 & 0.643510 \\ \mathrm{C} & -1.998720 & -0.167420 & -0.756120 \\ \mathrm{~N} & -3.581690 & 0.012770 & 0.796190 \\ \mathrm{~N} & -3.169370 & 0.054820 & -1.408900 \\ \mathrm{C} & -4.093140 & 0.172940 & -0.453080 \\ \mathrm{H} & 1.668240 & -2.258780 & 0.991270 \\ \mathrm{H} & -0.043780 & 1.713870 & 0.716340 \\ \mathrm{H} & -5.159690 & 0.383630 & -0.623480 \\ \mathrm{C} & -4.289870 & 0.069970 & 2.063510 \\ \mathrm{H} & -5.314530 & -0.335030 & 1.934530 \\ \mathrm{H} & -3.723410 & -0.538490 & 2.797400 \\ \mathrm{H} & -4.352890 & 1.114090 & 2.442260 \\ \mathrm{H} & -1.056010 & -0.313650 & -1.298200 \\ \mathrm{O} & 3.706850 & 0.926190 & -1.671130 \\ \mathrm{C} & 4.735680 & 0.055160 & -2.099450 \\ \mathrm{H} & 4.329990 & -0.821270 & -2.659210 \\ \mathrm{H} & 5.382010 & 0.649650 & -2.776450 \\ \mathrm{H} & 5.348460 & -0.315020 & -1.243010 \\ \mathrm{H} & 1.826910 & 2.405480 & -0.807600 \\ \mathrm{H} & 3.478300 & -1.608880 & -0.597670\end{array}$

cis- $\beta$

$E_{P B E / S V P}=-719.1400392556$ Hartree $\mathrm{NImag}=0$

$\begin{array}{lrrr}\text { C } & 1.023860 & 1.016290 & 0.468100 \\ \text { C } & 0.367860 & 1.867190 & 1.395650 \\ \text { C } & -0.532230 & 1.356300 & 2.326290 \\ \text { C } & -0.763380 & -0.038950 & 2.404830 \\ \text { C } & -0.074370 & -0.903830 & 1.523980 \\ \text { C } & 0.794780 & -0.378400 & 0.558120 \\ \text { N } & 2.013380 & 1.608340 & -0.348040 \\ \text { N } & 2.347970 & 1.190800 & -1.499570 \\ \mathrm{C} & 1.633470 & 0.314280 & -2.301660 \\ \mathrm{C} & 2.190240 & -0.606660 & -3.206120 \\ \mathrm{~N} & 0.246140 & 0.226750 & -2.512780 \\ \mathrm{~N} & 1.218350 & -1.261460 & -3.901360 \\ \mathrm{C} & 0.077770 & -0.723010 & -3.489940 \\ \mathrm{H} & 0.588380 & 2.945900 & 1.355240 \\ \mathrm{H} & 1.330370 & -1.067720 & -0.113360 \\ \mathrm{H} & 3.260110 & -0.824310 & -3.326800 \\ \mathrm{C} & -0.917930 & -0.939460 & -3.907270 \\ \mathrm{H} & -0.802920 & 1.088750 & -1.993030 \\ \mathrm{H} & -1.639370 & 1.111690 & -2.722540 \\ \mathrm{H} & -1.177760 & 0.732440 & -1.010090 \\ \mathrm{H} & -0.415690 & 2.120500 & -1.867640 \\ \mathrm{O} & -1.639790 & -0.444750 & 3.359610 \\ \mathrm{C} & -1.902720 & -1.828310 & 3.505410 \\ \mathrm{H} & -2.635820 & -1.917050 & 4.332330 \\ \mathrm{H} & -2.343640 & -2.265320 & 2.578140 \\ \mathrm{H} & -0.981370 & -2.398240 & 3.772670 \\ \mathrm{H} & -1.061040 & 2.012830 & 3.034970 \\ \mathrm{H} & -0.204640 & -1.994600 & 1.584870\end{array}$


trans- $\alpha$

$E_{P B E / S V P}=-719.1690336034$ Hartree NImag $=0$

$\begin{array}{lrrr}\mathrm{C} & -0.715650 & -0.033930 & -0.108390 \\ \mathrm{C} & -2.109820 & 0.056190 & -0.313230 \\ \mathrm{C} & -2.912310 & -1.089050 & -0.389250 \\ \mathrm{C} & -2.317800 & -2.363610 & -0.259390 \\ \mathrm{C} & -0.916800 & -2.465100 & -0.053380 \\ \mathrm{C} & -0.129370 & -1.322720 & 0.020970 \\ \mathrm{~N} & -0.020550 & 1.182370 & -0.051580 \\ \mathrm{~N} & 1.240290 & 1.082720 & 0.144570 \\ \mathrm{C} & 1.915720 & 2.275890 & 0.192860 \\ \mathrm{C} & 1.589180 & 3.640480 & 0.068410 \\ \mathrm{~N} & 3.294690 & 2.255670 & 0.413670 \\ \mathrm{~N} & 2.700650 & 4.413530 & 0.204400 \\ \mathrm{C} & 3.702430 & 3.558090 & 0.410070 \\ \mathrm{H} & -2.547900 & 1.062230 & -0.411470 \\ \mathrm{H} & 0.957660 & -1.388660 & 0.179470 \\ \mathrm{H} & 4.756310 & 3.834760 & 0.564200 \\ \mathrm{C} & 4.108540 & 1.070520 & 0.611390 \\ \mathrm{H} & 3.770370 & 0.507710 & 1.507090 \\ \mathrm{H} & 4.037350 & 0.397020 & -0.268390 \\ \mathrm{H} & 5.163340 & 1.383360 & 0.750940 \\ \mathrm{H} & 0.591060 & 4.058540 & -0.114700 \\ \mathrm{O} & -2.991770 & -3.540960 & -0.317230 \\ \mathrm{C} & -4.391700 & -3.518590 & -0.527120 \\ \mathrm{H} & -4.655300 & -3.041680 & -1.500890 \\ \mathrm{H} & -4.718360 & -4.577920 & -0.541730 \\ \mathrm{H} & -4.923890 & -2.983620 & 0.294930 \\ \mathrm{H} & -0.481430 & -3.472740 & 0.043240 \\ \mathrm{H} & -3.994960 & -0.980470 & -0.549450\end{array}$

trans- $\beta$

$E_{P B E / S V P}=-719.1691145475$ Hartree $\mathrm{NImag}=0$

$\begin{array}{lrrr}\mathrm{C} & -0.053040 & 0.240370 & -0.067210 \\ \mathrm{C} & 0.566640 & -1.001690 & -0.322150 \\ \mathrm{C} & -0.180400 & -2.182480 & -0.425820 \\ \mathrm{C} & -1.583450 & -2.132620 & -0.272400 \\ \mathrm{C} & -2.215310 & -0.887630 & -0.017320 \\ \mathrm{C} & -1.465620 & 0.278050 & 0.083670 \\ \mathrm{~N} & 0.793160 & 1.358770 & 0.021630 \\ \mathrm{~N} & 0.196050 & 2.468240 & 0.254240 \\ \mathrm{C} & 0.972230 & 3.587590 & 0.357160 \\ \mathrm{C} & 0.504070 & 4.889960 & 0.611810 \\ \mathrm{~N} & 2.368400 & 3.726790 & 0.248090 \\ \mathrm{~N} & 1.528110 & 5.782470 & 0.660360 \\ \mathrm{C} & 2.621570 & 5.056420 & 0.439690 \\ \mathrm{H} & 1.662350 & -1.019530 & -0.437450 \\ \mathrm{H} & -1.941360 & 1.250280 & 0.282150 \\ \mathrm{H} & -0.546530 & 5.176790 & 0.757420 \\ \mathrm{C} & 3.649700 & 5.450520 & 0.407080 \\ \mathrm{H} & 3.372130 & 2.706570 & -0.012200 \\ \mathrm{H} & 3.348750 & 1.922400 & 0.770920 \\ \mathrm{H} & 3.186470 & 2.211210 & -0.986350 \\ \mathrm{H} & 4.367890 & 3.198980 & -0.023550 \\ \mathrm{O} & -2.408800 & -3.208520 & -0.349460 \\ \mathrm{C} & -1.849030 & -4.483990 & -0.600980 \\ \mathrm{H} & -2.698990 & -5.195930 & -0.618480 \\ \mathrm{H} & -1.324130 & -4.518100 & -1.585160 \\ \mathrm{H} & -1.135370 & -4.787430 & 0.201560 \\ \mathrm{H} & 0.335850 & -3.133020 & -0.624460 \\ \mathrm{H} & -3.311060 & -0.878690 & 0.097390\end{array}$




\section{I.5.8 1-Methyl-4'-N,N-dimethylaminophenylazoimidazole (2c)}

\begin{tabular}{|c|c|c|c|c|c|c|c|}
\hline & & & & 0 & & & \\
\hline & $p=-738.5302$ & 93690 Hartr & & & $p=-738.5231$ & 243924 Hartre & \\
\hline & $=0$ & & & & $g=0$ & & \\
\hline C & 0.930420 & -0.689150 & 0.785620 & C & 0.930420 & -0.689150 & 0.785620 \\
\hline C & 1.959600 & -1.627250 & 0.531390 & C & 1.959600 & -1.627250 & 0.531390 \\
\hline C & 3.017010 & -1.340340 & -0.330840 & C & 3.017010 & -1.340340 & -0.330840 \\
\hline C & 3.136860 & -0.057640 & -0.943420 & C & 3.136860 & -0.057640 & -0.943420 \\
\hline C & 2.135660 & 0.910410 & -0.625120 & C & 2.135660 & 0.910410 & -0.625120 \\
\hline C & 1.057350 & 0.595290 & 0.200260 & C & 1.057350 & 0.595290 & 0.200260 \\
\hline $\mathrm{N}$ & -0.031350 & -1.045420 & 1.753420 & $\mathrm{~N}$ & -0.031350 & -1.045420 & 1.753420 \\
\hline $\mathrm{N}$ & -1.255570 & -0.709840 & 1.717330 & $\mathrm{~N}$ & -1.255570 & -0.709840 & 1.717330 \\
\hline C & -1.945610 & -0.187910 & 0.634730 & C & -1.945610 & -0.187910 & 0.634730 \\
\hline C & -1.844190 & -0.143550 & -0.775040 & C & -1.844190 & -0.143550 & -0.775040 \\
\hline N & -3.269110 & 0.209010 & 0.893150 & $\mathrm{~N}$ & -3.269110 & 0.209010 & 0.893150 \\
\hline $\mathrm{N}$ & -3.019610 & 0.249720 & -1.335650 & $\mathrm{~N}$ & -3.019610 & 0.249720 & -1.335650 \\
\hline C & -3.845750 & 0.468020 & -0.312260 & C & -3.845750 & 0.468020 & -0.312260 \\
\hline $\mathrm{H}$ & 1.897290 & -2.608500 & 1.028660 & $\mathrm{H}$ & 1.897290 & -2.608500 & 1.028660 \\
\hline $\mathrm{H}$ & 3.770920 & -2.118670 & -0.514470 & $\mathrm{H}$ & 3.770920 & -2.118670 & -0.514470 \\
\hline $\mathrm{H}$ & 2.204030 & 1.931950 & -1.025230 & $\mathrm{H}$ & 2.204030 & 1.931950 & -1.025230 \\
\hline $\mathrm{H}$ & 0.309560 & 1.372900 & 0.419970 & $\mathrm{H}$ & 0.309560 & 1.372900 & 0.419970 \\
\hline $\mathrm{H}$ & -4.882120 & 0.827950 & -0.396290 & $\mathrm{H}$ & -4.882120 & 0.827950 & -0.396290 \\
\hline C & -3.866930 & 0.322990 & 2.210950 & C & -3.866930 & 0.322990 & 2.210950 \\
\hline $\mathrm{H}$ & -4.951590 & 0.099090 & 2.146650 & $\mathrm{H}$ & -4.951590 & 0.099090 & 2.146650 \\
\hline $\mathrm{H}$ & -3.359770 & -0.403530 & 2.878510 & $\mathrm{H}$ & -3.359770 & -0.403530 & 2.878510 \\
\hline $\mathrm{H}$ & -3.728420 & 1.344130 & 2.631370 & $\mathrm{H}$ & -3.728420 & 1.344130 & 2.631370 \\
\hline $\mathrm{H}$ & -0.971490 & -0.401740 & -1.388190 & $\mathrm{H}$ & -0.971490 & -0.401740 & -1.388190 \\
\hline$N$ & 4.186790 & 0.243840 & -1.792310 & $\mathrm{~N}$ & 4.186790 & 0.243840 & -1.792310 \\
\hline C & 5.216780 & -0.744190 & -2.050260 & C & 5.216780 & -0.744190 & -2.050260 \\
\hline $\mathrm{H}$ & 4.796880 & -1.673080 & -2.504140 & $\mathrm{H}$ & 4.796880 & -1.673080 & -2.504140 \\
\hline $\mathrm{H}$ & 5.957410 & -0.325890 & -2.760290 & $\mathrm{H}$ & 5.957410 & -0.325890 & -2.760290 \\
\hline $\mathrm{H}$ & 5.762100 & -1.039360 & -1.120800 & $\mathrm{H}$ & 5.762100 & -1.039360 & -1.120800 \\
\hline C & 4.293300 & 1.567420 & -2.375680 & C & 4.293300 & 1.567420 & -2.375680 \\
\hline $\mathrm{H}$ & 5.172650 & 1.602250 & -3.048960 & $\mathrm{H}$ & 5.172650 & 1.602250 & -3.048960 \\
\hline $\mathrm{H}$ & 3.392580 & 1.827490 & -2.980770 & $\mathrm{H}$ & 3.392580 & 1.827490 & -2.980770 \\
\hline & 4.423290 & 2.363520 & -1.602380 & $\mathrm{H}$ & 4.423290 & 2.363520 & -1.60238 \\
\hline
\end{tabular}




\section{trans- $\alpha$}

$E_{P B E / S V P}=-738.5517782074$ Hartree

NImag $=0$

\begin{tabular}{lrrr}
$\mathrm{C}$ & -0.297400 & 0.587150 & -0.277810 \\
$\mathrm{C}$ & -1.636860 & 0.752200 & -0.703390 \\
$\mathrm{C}$ & -2.566680 & -0.281620 & -0.609940 \\
$\mathrm{C}$ & -2.195580 & -1.552270 & -0.077340 \\
$\mathrm{C}$ & -0.840480 & -1.713470 & 0.355060 \\
$\mathrm{C}$ & 0.077290 & -0.673680 & 0.255150 \\
$\mathrm{~N}$ & 0.544510 & 1.692870 & -0.419560 \\
$\mathrm{~N}$ & 1.755430 & 1.516640 & -0.036460 \\
$\mathrm{C}$ & 2.570010 & 2.612460 & -0.177690 \\
$\mathrm{C}$ & 2.433750 & 3.935370 & -0.640460 \\
$\mathrm{~N}$ & 3.907670 & 2.504900 & 0.208990 \\
$\mathrm{~N}$ & 3.617750 & 4.602580 & -0.544400 \\
$\mathrm{C}$ & 4.477970 & 3.722430 & -0.034080 \\
$\mathrm{H}$ & -1.924300 & 1.733190 & -1.114620 \\
$\mathrm{H}$ & -3.594320 & -0.100230 & -0.954320 \\
$\mathrm{H}$ & -0.505480 & -2.672240 & 0.776530 \\
$\mathrm{H}$ & 1.117290 & -0.807310 & 0.590260 \\
$\mathrm{H}$ & 5.539410 & 3.917260 & 0.182020 \\
$\mathrm{C}$ & 4.541370 & 1.321550 & 0.758910 \\
$\mathrm{H}$ & 4.046080 & 1.014860 & 1.704450 \\
$\mathrm{H}$ & 4.475700 & 0.474050 & 0.044240 \\
$\mathrm{H}$ & 5.607770 & 1.550760 & 0.960270 \\
$\mathrm{H}$ & 1.518490 & 4.399300 & -1.029870 \\
$\mathrm{~N}$ & -3.106420 & -2.587450 & 0.019010 \\
$\mathrm{C}$ & -4.476190 & -2.391130 & -0.417290 \\
$\mathrm{H}$ & -5.045970 & -3.330190 & -0.273660 \\
$\mathrm{H}$ & -4.990200 & -1.586170 & 0.161400 \\
$\mathrm{H}$ & -4.533860 & -2.118990 & -1.498150 \\
$\mathrm{H}$ & -2.705780 & -3.863060 & 0.582660 \\
& -3.569000 & -4.557370 & 0.568050 \\
\hline & -1.878920 & -4.335980 & 0.001070 \\
$\mathrm{H}$ & -363040 & -3.766410 & 1.640950
\end{tabular}

trans- $\beta$

$E_{P B E / S V P}=-738.5519143641$ Hartree NImag $=0$

$\begin{array}{lrrr}\text { C } & 0.139140 & 0.188940 & 0.020820 \\ \mathrm{C} & 0.799530 & -0.957120 & 0.520810 \\ \mathrm{C} & 0.190660 & -2.211340 & 0.518350 \\ \mathrm{C} & -1.130490 & -2.381490 & 0.007480 \\ \mathrm{C} & -1.794350 & -1.217680 & -0.495280 \\ \mathrm{C} & -1.175090 & 0.027320 & -0.487690 \\ \mathrm{~N} & 0.840620 & 1.399850 & 0.069060 \\ \mathrm{~N} & 0.208660 & 2.412840 & -0.401340 \\ \mathrm{C} & 0.853140 & 3.617930 & -0.373860 \\ \mathrm{C} & 0.333550 & 4.838450 & -0.842180 \\ \mathrm{~N} & 2.136860 & 3.940000 & 0.104530 \\ \mathrm{~N} & 1.223060 & 5.853920 & -0.671890 \\ \mathrm{C} & 2.283510 & 5.283960 & -0.106520 \\ \mathrm{H} & 1.820710 & -0.833620 & 0.916780 \\ \mathrm{H} & 0.749190 & -3.069240 & 0.918350 \\ \mathrm{H} & -2.814660 & -1.293680 & -0.898490 \\ \mathrm{H} & -1.692080 & 0.917880 & -0.876780 \\ \mathrm{H} & -0.658570 & 4.982240 & -1.291970 \\ \mathrm{H} & 3.209820 & 5.806880 & 0.179950 \\ \mathrm{C} & 3.135110 & 3.069520 & 0.704560 \\ \mathrm{H} & 2.739250 & 2.588960 & 1.621530 \\ \mathrm{H} & 3.426190 & 2.261400 & 0.003870 \\ \mathrm{H} & 4.023490 & 3.687900 & 0.956520 \\ \mathrm{~N} & -1.746420 & -3.618940 & -0.002670 \\ \mathrm{C} & -1.047020 & -4.780730 & 0.511960 \\ \mathrm{H} & -1.697640 & -5.672630 & 0.419440 \\ \mathrm{H} & -0.104340 & -4.985370 & -0.050690 \\ \mathrm{H} & -0.777940 & -4.664190 & 1.589270 \\ \mathrm{C} & -3.089680 & -3.761400 & -0.532240 \\ \mathrm{H} & -3.402250 & -4.821900 & -0.459090 \\ \mathrm{H} & -3.830940 & -3.147350 & 0.033110 \\ \mathrm{H} & -3.151030 & -3.461320 & -1.605670\end{array}$


I.5.9 1-Methyl-2',5'-dimethyl-4'-methoxyphenylazoimidazole (2d)

\section{cis- $\alpha$}

$E_{P B E / S V P}=-797.5971301712$ Hartree

NImag $=0$

$\begin{array}{lrrr}\mathrm{C} & 0.983500 & -0.442980 & 0.833740 \\ \mathrm{C} & 1.765600 & -1.489730 & 0.309960 \\ \mathrm{C} & 2.723460 & -1.249700 & -0.692500 \\ \mathrm{C} & 2.923320 & 0.084270 & -1.123430 \\ \mathrm{C} & 2.161830 & 1.158430 & -0.595850 \\ \mathrm{C} & 1.189660 & 0.877010 & 0.376420 \\ \mathrm{~N} & 0.102380 & -0.719920 & 1.915550 \\ \mathrm{~N} & -1.143850 & -0.507510 & 1.858260 \\ \mathrm{C} & -1.853420 & -0.166970 & 0.718440 \\ \mathrm{C} & -1.690640 & -0.172490 & -0.686960 \\ \mathrm{~N} & -3.219330 & 0.104200 & 0.907190 \\ \mathrm{~N} & -2.871590 & 0.077010 & -1.309590 \\ \mathrm{C} & -3.761470 & 0.250950 & -0.329680 \\ \mathrm{H} & 1.600400 & -2.513270 & 0.684180 \\ \mathrm{H} & 0.584300 & 1.697220 & 0.795780 \\ \mathrm{H} & -4.825280 & 0.492580 & -0.474560 \\ \mathrm{C} & -3.885970 & 0.229740 & 2.192570 \\ \mathrm{H} & -4.921240 & -0.160820 & 2.113890 \\ \mathrm{H} & -3.307870 & -0.356160 & 2.935160 \\ \mathrm{H} & -3.918100 & 1.290730 & 2.525240 \\ \mathrm{H} & -0.770420 & -0.366360 & -1.251850 \\ \mathrm{C} & 3.517350 & -2.382430 & -1.297790 \\ \mathrm{H} & 3.775850 & -2.162700 & -2.355160 \\ \mathrm{H} & 4.474640 & -2.554370 & -0.752510 \\ \mathrm{H} & 2.946680 & -3.334530 & -1.260200 \\ \mathrm{C} & 2.388610 & 2.562720 & -1.101380 \\ \mathrm{H} & 3.385810 & 2.955240 & -0.797840 \\ \mathrm{H} & 2.361010 & 2.596050 & -2.212260 \\ \mathrm{H} & 1.619230 & 3.258950 & -0.706680 \\ \mathrm{O} & 3.834740 & 0.356880 & -2.126780 \\ \mathrm{C} & 5.181660 & 0.519160 & -1.704580 \\ \mathrm{H} & 5.593490 & -0.412290 & -1.247480 \\ \mathrm{H} & 5.773120 & 0.765190 & -2.611310 \\ & 5.287440 & 1.350110 & -0.966100\end{array}$

\section{cis- $\beta$}

$E_{P B E / S V P}=-797.5892489900$ Hartree NImag $=0$

$\begin{array}{lrrr}\mathrm{C} & 0.544920 & 0.458670 & 1.190080 \\ \mathrm{C} & -0.127270 & 1.266640 & 2.136070 \\ \mathrm{C} & -1.149660 & 0.748550 & 2.942730 \\ \mathrm{C} & -1.456030 & -0.633220 & 2.835680 \\ \mathrm{C} & -0.780260 & -1.476510 & 1.916870 \\ \mathrm{C} & 0.208490 & -0.912260 & 1.092560 \\ \mathrm{~N} & 1.647920 & 1.040200 & 0.521120 \\ \mathrm{~N} & 2.021920 & 0.745670 & -0.654330 \\ \mathrm{C} & 1.291820 & 0.068410 & -1.616900 \\ \mathrm{C} & 1.842320 & -0.752680 & -2.617570 \\ \mathrm{~N} & -0.081790 & 0.120780 & -1.915810 \\ \mathrm{~N} & 0.877020 & -1.222750 & -3.454700 \\ \mathrm{C} & -0.248780 & -0.661120 & -3.030730 \\ \mathrm{H} & 0.169170 & 2.325190 & 2.219260 \\ \mathrm{H} & 0.742290 & -1.557290 & 0.375930 \\ \mathrm{H} & 2.900620 & -1.032450 & -2.709100 \\ \mathrm{C} & -1.226960 & -0.737050 & -3.530510 \\ \mathrm{H} & -1.104820 & 0.975650 & -1.335000 \\ \mathrm{H} & -1.889830 & 1.158240 & -2.098040 \\ \mathrm{H} & -1.561420 & 0.513600 & -0.434230 \\ \mathrm{H} & -0.661950 & 1.949510 & -1.042830 \\ \mathrm{C} & -1.135850 & -2.940340 & 1.810760 \\ \mathrm{H} & -0.564330 & -3.558150 & 2.541550 \\ \mathrm{H} & -2.215000 & -3.102310 & 2.017250 \\ \mathrm{H} & -0.901980 & -3.335200 & 0.799360 \\ \mathrm{C} & -1.915500 & 1.617370 & 3.910920 \\ \mathrm{H} & -3.011330 & 1.540380 & 3.737950 \\ \mathrm{H} & -1.742120 & 1.311230 & 4.967260 \\ \mathrm{H} & -1.615470 & 2.681780 & 3.815100 \\ \mathrm{O} & -2.480680 & -1.136620 & 3.611150 \\ \mathrm{C} & -2.088010 & -1.783480 & 4.815470 \\ \mathrm{H} & -3.021830 & -2.091100 & 5.330480 \\ \mathrm{H} & -1.467470 & -2.690280 & 4.621740 \\ \mathrm{H} & -1.513030 & -1.097930 & 5.482840\end{array}$


trans- $\alpha$

$E_{P B E / S V P}=-797.6172430495$ Hartree $\mathrm{N}$ Imag $=0$

\begin{tabular}{|c|c|c|c|}
\hline C & -0.991190 & -0.585320 & -0.063510 \\
\hline C & -2.392290 & -0.417770 & -0.070260 \\
\hline C & -3.265430 & -1.517670 & -0.069300 \\
\hline C & -2.694900 & -2.814270 & -0.071760 \\
\hline C & -1.286670 & -3.015230 & -0.042210 \\
\hline C & -0.450390 & -1.893920 & -0.040790 \\
\hline$N$ & -0.236680 & 0.601480 & -0.068960 \\
\hline$N$ & 1.031130 & 0.441790 & -0.021030 \\
\hline C & 1.762910 & 1.601730 & -0.041790 \\
\hline C & 1.488060 & 2.980390 & -0.133560 \\
\hline$N$ & 3.155070 & 1.517260 & 0.033320 \\
\hline$N$ & 2.641520 & 3.700920 & -0.115930 \\
\hline C & 3.619230 & 2.799300 & -0.014150 \\
\hline $\mathrm{H}$ & -2.782250 & 0.613200 & -0.062270 \\
\hline $\mathrm{H}$ & 0.644600 & -2.007600 & -0.020360 \\
\hline $\mathrm{H}$ & 4.694880 & 3.027430 & 0.030190 \\
\hline C & 3.930220 & 0.295290 & 0.148330 \\
\hline $\mathrm{H}$ & 3.659810 & -0.254100 & 1.074720 \\
\hline $\mathrm{H}$ & 3.738760 & -0.371160 & -0.718610 \\
\hline $\mathrm{H}$ & 5.006740 & 0.559850 & 0.178960 \\
\hline $\mathrm{H}$ & 0.496440 & 3.444180 & -0.213640 \\
\hline C & -0.735740 & -4.419930 & 0.008040 \\
\hline $\mathrm{H}$ & -1.018860 & -5.009600 & -0.892920 \\
\hline $\mathrm{H}$ & 0.372450 & -4.409190 & 0.073210 \\
\hline $\mathrm{H}$ & -1.135460 & -4.976640 & 0.884280 \\
\hline C & -4.763220 & -1.328780 & -0.036000 \\
\hline $\mathrm{H}$ & -5.030150 & -0.371100 & 0.45927 \\
\hline $\mathrm{H}$ & -5.202740 & -1.298540 & -1.060050 \\
\hline $\mathrm{H}$ & -5.256040 & -2.160760 & 0.510370 \\
\hline $\mathrm{O}$ & -3.504220 & -3.930100 & -0.02440 \\
\hline C & -4.062220 & -4.348700 & -1.262880 \\
\hline $\mathrm{H}$ & -3.268720 & -4.595130 & -2.00856 \\
\hline $\mathrm{H}$ & -4.656500 & -5.262080 & -1.05155 \\
\hline $\mathrm{H}$ & -4.734350 & -3.575820 & -1.70537 \\
\hline
\end{tabular}

trans- $\beta$

$E_{P B E / S V P}=-797.6175565840$ Hartree NImag $=0$

$\begin{array}{lrrr}\mathrm{C} & 0.093480 & 0.116290 & 0.155110 \\ \mathrm{C} & 0.796880 & -1.105870 & 0.119110 \\ \mathrm{C} & 0.126190 & -2.340070 & 0.105410 \\ \mathrm{C} & -1.290100 & -2.323350 & 0.125050 \\ \mathrm{C} & -2.025720 & -1.107650 & 0.187560 \\ \mathrm{C} & -1.320950 & 0.101060 & 0.197770 \\ \mathrm{~N} & 0.881410 & 1.285050 & 0.162580 \\ \mathrm{~N} & 0.218070 & 2.379560 & 0.188770 \\ \mathrm{C} & 0.931630 & 3.544120 & 0.196840 \\ \mathrm{C} & 0.384940 & 4.840760 & 0.219580 \\ \mathrm{~N} & 2.324570 & 3.745560 & 0.183670 \\ \mathrm{~N} & 1.359500 & 5.787750 & 0.220370 \\ \mathrm{C} & 2.499740 & 5.100630 & 0.198930 \\ \mathrm{H} & 1.898770 & -1.067200 & 0.113320 \\ \mathrm{H} & -1.850250 & 1.065450 & 0.244530 \\ \mathrm{H} & -0.686240 & 5.084900 & 0.234560 \\ \mathrm{C} & 3.507930 & 5.544690 & 0.193590 \\ \mathrm{H} & 3.392250 & 2.757640 & 0.161430 \\ \mathrm{H} & 3.333450 & 2.095600 & 1.048380 \\ \mathrm{H} & 3.316330 & 2.116950 & -0.739680 \\ \mathrm{H} & 4.361990 & 3.299550 & 0.158900 \\ \mathrm{C} & 0.884850 & -3.645810 & 0.098870 \\ \mathrm{H} & 1.046370 & -4.028680 & -0.935850 \\ \mathrm{H} & 1.887830 & -3.524600 & 0.560240 \\ \mathrm{H} & 0.329360 & -4.429700 & 0.656230 \\ \mathrm{C} & -3.532710 & -1.149300 & 0.263980 \\ \mathrm{H} & -3.950570 & -0.130260 & 0.400430 \\ \mathrm{H} & -3.982390 & -1.580710 & -0.658860 \\ \mathrm{H} & -3.873190 & -1.789030 & 1.107540 \\ \mathrm{O} & -1.995240 & -3.509020 & 0.165680 \\ \mathrm{C} & -2.214230 & -4.144840 & -1.086630 \\ \mathrm{H} & -2.815760 & -5.054710 & -0.880860 \\ \mathrm{H} & -2.779380 & -3.486670 & -1.789150 \\ \mathrm{H} & -1.258800 & -4.448120 & -1.577370\end{array}$




\section{I.5.10 1-Methyl-2',5'-di-tert-butyl-4'-methoxyphenylazoimidazole (2e)}

\section{cis- $\alpha$}

$E_{P B E / S V P}=-1032.917950750$ Hartree

NImag $=0$

\begin{tabular}{|c|c|c|c|}
\hline C & 1.153400 & -0.373980 & 0.846120 \\
\hline C & 1.882430 & -1.431930 & 0.287070 \\
\hline C & 2.787170 & -1.236790 & -0.778180 \\
\hline C & 3.049850 & 0.113080 & -1.156030 \\
\hline C & 2.260470 & 1.207010 & -0.668960 \\
\hline C & 1.320350 & 0.929040 & 0.335210 \\
\hline$N$ & 0.348480 & -0.630320 & 1.989590 \\
\hline$N$ & -0.889240 & -0.369910 & 2.037170 \\
\hline C & -1.682320 & 0.001510 & 0.963320 \\
\hline C & -1.646970 & -0.013500 & -0.450900 \\
\hline$N$ & -3.015500 & 0.321230 & 1.271450 \\
\hline$N$ & -2.869580 & 0.276090 & -0.967210 \\
\hline C & -3.661180 & 0.484310 & 0.087200 \\
\hline $\mathrm{H}$ & 1.700790 & -2.438620 & 0.693530 \\
\hline $\mathrm{H}$ & 0.704310 & 1.731710 & 0.761470 \\
\hline $\mathrm{H}$ & -4.724070 & 0.764330 & 0.037060 \\
\hline C & -3.560130 & 0.469410 & 2.610550 \\
\hline $\mathrm{H}$ & -4.609330 & 0.109390 & 2.627390 \\
\hline $\mathrm{H}$ & -2.934410 & -0.131910 & 3.300360 \\
\hline $\mathrm{H}$ & -3.531660 & 1.531610 & 2.939780 \\
\hline $\mathrm{H}$ & -0.788280 & -0.242280 & -1.094350 \\
\hline C & 3.366180 & -2.514540 & -1.454720 \\
\hline C & 2.350380 & 2.631880 & -1.278780 \\
\hline $\mathrm{O}$ & 4.080590 & 0.406210 & -2.032360 \\
\hline C & 5.377570 & 0.384660 & -1.447550 \\
\hline $\mathrm{H}$ & 5.652230 & -0.626440 & -1.067200 \\
\hline $\mathrm{H}$ & 6.090830 & 0.674210 & -2.247340 \\
\hline $\mathrm{H}$ & 5.459070 & 1.108470 & -0.603480 \\
\hline C & 2.187080 & -3.487920 & -1.738640 \\
\hline $\mathrm{H}$ & 1.667070 & -3.821840 & -0.816570 \\
\hline $\mathrm{H}$ & 1.431730 & -3.013690 & -2.403670 \\
\hline $\mathrm{H}$ & 2.570000 & -4.399590 & -2.248490 \\
\hline C & 4.049720 & -2.285210 & -2.823800 \\
\hline $\mathrm{H}$ & 5.027720 & -1.771670 & -2.751760 \\
\hline $\mathrm{H}$ & 4.238640 & -3.277520 & -3.291490 \\
\hline $\mathrm{H}$ & 3.405780 & -1.700180 & -3.513010 \\
\hline C & 4.360980 & -3.217140 & -0.498060 \\
\hline $\mathrm{H}$ & 5.242580 & -2.575330 & -0.279190 \\
\hline $\mathrm{H}$ & 3.883220 & -3.472200 & 0.473460 \\
\hline $\mathrm{H}$ & 4.733360 & -4.162350 & -0.953760 \\
\hline$C$ & 3.727570 & 3.297380 & -1.036950 \\
\hline $\mathrm{H}$ & 4.531230 & 2.816650 & -1.627730 \\
\hline $\mathrm{H}$ & 3.685280 & 4.366310 & -1.345050 \\
\hline $\mathrm{H}$ & 4.007590 & 3.267430 & 0.039490 \\
\hline$C$ & 2.078010 & 2.541980 & -2.802460 \\
\hline $\mathrm{H}$ & 2.129840 & 3.556100 & -3.259680 \\
\hline $\mathrm{H}$ & 2.823290 & 1.893020 & -3.305930 \\
\hline $\mathrm{H}$ & 1.062250 & 2.132110 & -2.997970 \\
\hline$C$ & 1.283770 & 3.572960 & -0.675220 \\
\hline $\mathrm{H}$ & 0.251480 & 3.186950 & -0.822350 \\
\hline $\mathrm{H}$ & 1.442500 & 3.748320 & 0.411860 \\
\hline $\mathrm{H}$ & 1.343160 & 4.561210 & -1.181230 \\
\hline
\end{tabular}

cis- $\beta$

$E_{P B E / S V P}=-1032.909522557$ Hartree NImag $=0$

\begin{tabular}{|c|c|c|c|}
\hline C & 1.599780 & 0.940390 & -0.452540 \\
\hline C & 0.881550 & 1.762000 & 0.438070 \\
\hline C & -0.174150 & 1.267180 & 1.217060 \\
\hline C & -0.442880 & -0.141470 & 1.149640 \\
\hline C & 0.214310 & -0.992430 & 0.202020 \\
\hline C & 1.241910 & -0.416390 & -0.570640 \\
\hline$N$ & 2.745620 & 1.503840 & -1.063100 \\
\hline N & 3.184540 & 1.195560 & -2.212320 \\
\hline C & 2.503720 & 0.517470 & -3.211190 \\
\hline C & 3.098430 & -0.324490 & -4.167990 \\
\hline N & 1.153290 & 0.595210 & -3.596200 \\
\hline$N$ & 2.177650 & -0.782030 & -5.060960 \\
\hline C & 1.039780 & -0.193670 & -4.713820 \\
\hline $\mathrm{H}$ & 1.190500 & 2.814570 & 0.493180 \\
\hline $\mathrm{H}$ & 1.803040 & -1.038770 & -1.279630 \\
\hline $\mathrm{H}$ & 4.154440 & -0.626010 & -4.191610 \\
\hline C & 0.093860 & -0.252670 & -5.274270 \\
\hline $\mathrm{H}$ & 0.114040 & 1.473320 & -3.083550 \\
\hline $\mathrm{H}$ & -0.618810 & 1.667710 & -3.894130 \\
\hline $\mathrm{H}$ & -0.406200 & 1.025270 & -2.210290 \\
\hline $\mathrm{H}$ & 0.557860 & 2.439590 & -2.768320 \\
\hline C & -0.137740 & -2.486750 & -0.061240 \\
\hline C & -1.016140 & 2.241470 & 2.083940 \\
\hline $\mathrm{O}$ & -1.415340 & -0.627160 & 2.002220 \\
\hline C & -1.021240 & -1.375910 & 3.145780 \\
\hline $\mathrm{H}$ & -1.716400 & -1.114870 & 3.973560 \\
\hline $\mathrm{H}$ & -1.102940 & -2.472510 & 2.963840 \\
\hline $\mathrm{H}$ & 0.019630 & -1.134330 & 3.456440 \\
\hline C & 0.689730 & -3.402910 & 0.876610 \\
\hline $\mathrm{H}$ & 0.483180 & -3.219300 & 1.950470 \\
\hline $\mathrm{H}$ & 0.463890 & -4.472920 & 0.665470 \\
\hline $\mathrm{H}$ & 1.778990 & -3.245250 & 0.714210 \\
\hline C & 0.228510 & -2.888820 & -1.515270 \\
\hline $\mathrm{H}$ & -0.237060 & -2.207730 & -2.261220 \\
\hline $\mathrm{H}$ & 1.323150 & -2.908530 & -1.701980 \\
\hline $\mathrm{H}$ & -0.145040 & -3.916910 & -1.714250 \\
\hline C & -1.653070 & -2.775110 & 0.078120 \\
\hline $\mathrm{H}$ & -1.852960 & -3.833380 & -0.201340 \\
\hline $\mathrm{H}$ & -2.047960 & -2.607910 & 1.096820 \\
\hline $\mathrm{H}$ & -2.239400 & -2.127530 & -0.610850 \\
\hline C & -0.604590 & 3.711800 & 1.843750 \\
\hline $\mathrm{H}$ & 0.437910 & 3.920790 & 2.169520 \\
\hline $\mathrm{H}$ & -0.701620 & 4.004530 & 0.775590 \\
\hline $\mathrm{H}$ & -1.272880 & 4.376360 & 2.433700 \\
\hline C & -0.828470 & 1.968990 & 3.596890 \\
\hline $\mathrm{H}$ & -1.259620 & 0.999370 & 3.910040 \\
\hline $\mathrm{H}$ & 0.249130 & 1.981440 & 3.872970 \\
\hline $\mathrm{H}$ & -1.338450 & 2.763010 & 4.187780 \\
\hline C & -2.513470 & 2.119390 & 1.702580 \\
\hline $\mathrm{H}$ & -2.890910 & 1.089670 & 1.863510 \\
\hline $\mathrm{H}$ & -3.121150 & 2.819570 & 2.319300 \\
\hline $\mathrm{H}$ & -2.669950 & 2.387250 & 0.633640 \\
\hline
\end{tabular}




\section{trans- $\alpha$}

$E_{P B E / S V P}=-1032.937999150$ Hartree

NImag $=0$

\begin{tabular}{|c|c|c|c|}
\hline C & -1.034030 & -0.634860 & -0.148690 \\
\hline C & -2.431340 & -0.490610 & -0.141530 \\
\hline C & -3.308430 & -1.590700 & -0.101320 \\
\hline$C$ & -2.706320 & -2.887630 & -0.142820 \\
\hline C & -1.282840 & -3.070710 & -0.035640 \\
\hline C & -0.476150 & -1.927110 & -0.054990 \\
\hline $\mathrm{N}$ & -0.297780 & 0.561680 & -0.209960 \\
\hline$N$ & 0.972360 & 0.423170 & -0.164090 \\
\hline C & 1.687690 & 1.591290 & -0.238410 \\
\hline C & 1.392410 & 2.961040 & -0.383660 \\
\hline$N$ & 3.081370 & 1.529690 & -0.169370 \\
\hline$N$ & 2.535480 & 3.698150 & -0.402 \\
\hline C & 3.526640 & 2.815380 & -0.271370 \\
\hline $\mathrm{H}$ & -2.806120 & 0.541900 & -0.154600 \\
\hline $\mathrm{H}$ & 0.617890 & -1.991040 & -0.003570 \\
\hline $\mathrm{H}$ & 4.599170 & 3.060250 & -0.243530 \\
\hline C & 3.875390 & 0.324940 & -0.010530 \\
\hline $\mathrm{H}$ & 3.619360 & -0.190700 & 0.939100 \\
\hline $\mathrm{H}$ & 3.688550 & -0.378930 & -0.848460 \\
\hline $\mathrm{H}$ & 4.948040 & 0.606330 & 0.001950 \\
\hline $\mathrm{H}$ & 0.393770 & 3.407240 & -0.475240 \\
\hline C & -0.636300 & -4.471060 & $0.140 s$ \\
\hline C & -4.833560 & -1.305010 & 0.01472 \\
\hline $\mathrm{O}$ & -3.469320 & -4.036040 & -0.217880 \\
\hline C & -4.066720 & -4.383180 & -1.461140 \\
\hline $\mathrm{H}$ & -4.049680 & -5.492230 & -1.541690 \\
\hline $\mathrm{H}$ & -5.129610 & -4.052070 & -1.512780 \\
\hline $\mathrm{H}$ & -3.507820 & -3.948910 & -2.320100 \\
\hline C & -1.242990 & -5.179770 & 1.378 \\
\hline $\mathrm{H}$ & -2.338020 & -5.313920 & 1.273550 \\
\hline $\mathrm{H}$ & -0.775170 & -6.182020 & 1.509510 \\
\hline $\mathrm{H}$ & -1.048990 & -4.590330 & 2.301820 \\
\hline C & -0.825690 & -5.348890 & -1.121060 \\
\hline $\mathrm{H}$ & -0.244010 & -6.292220 & -1.014850 \\
\hline $\mathrm{H}$ & -1.883180 & -5.631130 & -1.284480 \\
\hline $\mathrm{H}$ & -0.456240 & -4.824730 & -2.030570 \\
\hline C & 0.886410 & -4.361400 & 0.381530 \\
\hline $\mathrm{H}$ & 1.422540 & -3.933030 & -0.493330 \\
\hline $\mathrm{H}$ & 1.124710 & -3.743480 & 1.274500 \\
\hline $\mathrm{H}$ & 1.300730 & -5.378360 & 0.558760 \\
\hline C & -5.579620 & -2.368510 & 0.856800 \\
\hline $\mathrm{H}$ & -6.642640 & -2.061910 & 0.976330 \\
\hline $\mathrm{H}$ & -5.563050 & -3.381340 & 0.414630 \\
\hline $\mathrm{H}$ & -5.133130 & -2.445990 & 1.872620 \\
\hline C & -5.465160 & -1.171820 & -1.394520 \\
\hline $\mathrm{H}$ & -4.973760 & -0.353560 & -1.965490 \\
\hline $\mathrm{H}$ & -5.374270 & -2.098670 & -1.99638 \\
\hline $\mathrm{H}$ & -6.548220 & -0.927190 & -1.308800 \\
\hline C & -5.072980 & 0.044890 & 0.742780 \\
\hline $\mathrm{H}$ & -4.551750 & 0.077750 & 1.72423 \\
\hline $\mathrm{H}$ & -4.744850 & 0.922530 & 0.1465 \\
\hline $\mathrm{H}$ & -6.162270 & 0.172340 & 0.9274 \\
\hline
\end{tabular}

trans- $\beta$

$E_{P B E / S V P}=-1032.938097054$ Hartree $\mathrm{NImag}=0$

\begin{tabular}{|c|c|c|c|}
\hline C & 0.623010 & 0.984970 & 0.026620 \\
\hline$c$ & 1.340320 & -0.222930 & 0.101470 \\
\hline C & 0.696000 & -1.469710 & 0.100100 \\
\hline$C$ & -0.732750 & -1.470360 & -0.011870 \\
\hline C & -1.500590 & -0.257940 & 0.047400 \\
\hline C & -0.783600 & 0.949400 & 0.032440 \\
\hline $\mathrm{N}$ & 1.392340 & 2.166210 & -0.003610 \\
\hline $\mathrm{N}$ & 0.712110 & 3.248500 & -0.064830 \\
\hline C & 1.403690 & 4.425700 & -0.099870 \\
\hline C & 0.832480 & 5.710390 & -0.162220 \\
\hline $\mathrm{N}$ & 2.792270 & 4.654150 & -0.085710 \\
\hline$N$ & 1.788770 & 6.675640 & -0.185330 \\
\hline C & 2.941790 & 6.011510 & -0.138910 \\
\hline $\mathrm{H}$ & 2.434050 & -0.139570 & 0.155290 \\
\hline $\mathrm{H}$ & -1.295300 & 1.920650 & 0.041360 \\
\hline $\mathrm{H}$ & -0.243080 & 5.933610 & -0.188940 \\
\hline C & 3.941320 & 6.474750 & -0.141870 \\
\hline $\mathrm{H}$ & 3.878280 & 3.687730 & -0.031620 \\
\hline $\mathrm{H}$ & 3.811670 & 3.071960 & 0.887430 \\
\hline $\mathrm{H}$ & 3.835430 & 2.999500 & -0.899440 \\
\hline $\mathrm{H}$ & 4.837610 & 4.247760 & -0.041950 \\
\hline C & 1.524780 & -2.772030 & 0.260150 \\
\hline C & -3.051380 & -0.182770 & 0.157910 \\
\hline $\mathrm{O}$ & -1.343700 & -2.705940 & -0.103030 \\
\hline C & -1.882600 & -3.117360 & -1.353180 \\
\hline $\mathrm{H}$ & -1.709940 & -4.212000 & -1.448760 \\
\hline $\mathrm{H}$ & -2.981600 & -2.938130 & -1.404350 \\
\hline $\mathrm{H}$ & -1.388660 & -2.597770 & -2.204300 \\
\hline C & -3.651020 & -1.343370 & 0.988840 \\
\hline $\mathrm{H}$ & -4.745970 & -1.183650 & 1.105660 \\
\hline $\mathrm{H}$ & -3.496520 & -2.341300 & 0.539440 \\
\hline $\mathrm{H}$ & -3.201870 & -1.367990 & 2.006090 \\
\hline C & -3.476660 & 1.117520 & 0.890720 \\
\hline $\mathrm{H}$ & -2.971410 & 1.214780 & 1.876510 \\
\hline $\mathrm{H}$ & -3.266810 & 2.035150 & 0.301580 \\
\hline $\mathrm{H}$ & -4.574120 & 1.093970 & 1.068340 \\
\hline C & -3.686680 & -0.127080 & -1.255200 \\
\hline $\mathrm{H}$ & -3.309710 & 0.756160 & -1.816440 \\
\hline $\mathrm{H}$ & -3.466130 & -1.027630 & -1.863180 \\
\hline $\mathrm{H}$ & -4.793560 & -0.035000 & -1.175120 \\
\hline C & 3.018400 & -2.463880 & 0.510350 \\
\hline $\mathrm{H}$ & 3.496210 & -1.955650 & -0.355480 \\
\hline $\mathrm{H}$ & 3.170670 & -1.833490 & 1.413560 \\
\hline $\mathrm{H}$ & 3.563630 & -3.419100 & 0.674820 \\
\hline C & 1.013900 & -3.572380 & 1.485150 \\
\hline $\mathrm{H}$ & -0.052900 & -3.851180 & 1.372330 \\
\hline $\mathrm{H}$ & 1.611870 & -4.503940 & 1.606840 \\
\hline $\mathrm{H}$ & 1.122210 & -2.973970 & 2.417020 \\
\hline C & 1.458500 & -3.649530 & -1.013890 \\
\hline $\mathrm{H}$ & 0.447300 & -4.063280 & -1.188960 \\
\hline $\mathrm{H}$ & 1.761630 & -3.069480 & -1.913960 \\
\hline $\mathrm{H}$ & 2.156320 & -4.511600 & -0.915400 \\
\hline
\end{tabular}


I.6 XYZ Coordinates of the PBE/SVP optimized Complexes

\section{I.6.1 Ni-tetrakis(pentafluorophenyl)porphyrin (NiTPPF 20$)$}

\section{NiTPPF $_{20}$}

$E_{P B E} / \mathrm{SVP}=-5398.245789419$ Hartree $\mathrm{NImag}=0$

\begin{tabular}{|c|c|c|c|}
\hline C & -1.039060 & -0.362540 & 2.768340 \\
\hline$N$ & 0.000000 & 0.000000 & 1.929800 \\
\hline C & -0.635480 & -0.254800 & 4.152410 \\
\hline C & 0.635480 & 0.254800 & 4.152410 \\
\hline C & 1.039060 & 0.362540 & 2.768340 \\
\hline C & -2.349800 & -0.635580 & 2.350540 \\
\hline C & -2.762280 & -0.365800 & 1.037210 \\
\hline C & -4.146820 & -0.251040 & 0.637610 \\
\hline C & -4.146820 & 0.251040 & -0.637610 \\
\hline C & -2.762280 & 0.365800 & -1.037210 \\
\hline $\mathrm{N}$ & -1.924810 & 0.000000 & 0.000000 \\
\hline $\mathrm{Ni}$ & 0.000000 & 0.000000 & 0.000000 \\
\hline$N$ & 1.924810 & 0.000000 & 0.000000 \\
\hline C & 2.762280 & -0.365800 & -1.037210 \\
\hline C & 4.146820 & -0.251040 & -0.637610 \\
\hline C & 4.146820 & 0.251040 & 0.637610 \\
\hline C & 2.762280 & 0.365800 & 1.037210 \\
\hline C & -2.349800 & 0.635580 & -2.350540 \\
\hline C & -1.039060 & 0.362540 & -2.768340 \\
\hline C & -0.635480 & 0.254800 & -4.152410 \\
\hline C & 0.635480 & -0.254800 & -4.152410 \\
\hline C & 1.039060 & -0.362540 & -2.768340 \\
\hline$N$ & 0.000000 & 0.000000 & -1.929800 \\
\hline C & 2.349800 & -0.635580 & -2.350540 \\
\hline C & 2.349800 & 0.635580 & 2.350540 \\
\hline $\mathrm{H}$ & -5.006740 & 0.487190 & -1.275960 \\
\hline $\mathrm{H}$ & -5.006740 & -0.487190 & 1.275960 \\
\hline $\mathrm{H}$ & -1.264900 & 0.501130 & -5.015460 \\
\hline $\mathrm{H}$ & 1.264900 & -0.501130 & -5.015460 \\
\hline $\mathrm{H}$ & 5.006740 & 0.487190 & 1.275960 \\
\hline $\mathrm{H}$ & 5.006740 & -0.487190 & -1.275960 \\
\hline $\mathrm{H}$ & -1.264900 & -0.501130 & 5.015460 \\
\hline $\mathrm{H}$ & 1.264900 & 0.501130 & 5.015460 \\
\hline C & -3.362670 & -1.098680 & 3.342960 \\
\hline C & 3.362670 & 1.098680 & 3.342960 \\
\hline C & -3.362670 & 1.098680 & -3.342960 \\
\hline C & 3.362670 & -1.098680 & -3.342960 \\
\hline C & 3.795070 & -2.439320 & -3.342850 \\
\hline C & 4.744150 & -2.908990 & -4.265610 \\
\hline C & 5.284020 & -2.023030 & -5.213200 \\
\hline C & 4.876940 & -0.677700 & -5.230750 \\
\hline C & 3.925960 & -0.231340 & -4.298080 \\
\hline $\mathrm{F}$ & 3.294950 & -3.302250 & -2.454020 \\
\hline $\mathrm{F}$ & 5.130880 & -4.185950 & -4.252360 \\
\hline $\mathrm{F}$ & 6.182370 & -2.460390 & -6.094600 \\
\hline $\mathrm{F}$ & 5.395120 & 0.164920 & -6.126810 \\
\hline $\mathrm{F}$ & 3.559020 & 1.054240 & -4.333320 \\
\hline C & 3.925960 & 0.231340 & 4.298080 \\
\hline C & 4.876940 & 0.677700 & 5.230750 \\
\hline C & 5.284020 & 2.023030 & 5.213200 \\
\hline C & 4.744150 & 2.908990 & 4.265610 \\
\hline
\end{tabular}




$\begin{array}{lrrr}\text { C } & 3.795070 & 2.439320 & 3.342850 \\ \text { F } & 3.559020 & -1.054240 & 4.333320 \\ \text { F } & 5.395120 & -0.164920 & 6.126810 \\ \text { F } & 6.182370 & 2.460390 & 6.094600 \\ \text { F } & 5.130880 & 4.185950 & 4.252360 \\ \text { F } & 3.294950 & 3.302250 & 2.454020 \\ \text { C } & -3.925960 & 0.231340 & -4.298080 \\ \text { C } & -4.876940 & 0.677700 & -5.230750 \\ \text { C } & -5.284020 & 2.023030 & -5.213200 \\ \text { C } & -4.744150 & 2.908990 & -4.265610 \\ \text { C } & -3.795070 & 2.439320 & -3.342850 \\ \text { F } & -3.559020 & -1.054240 & -4.333320 \\ \text { F } & -5.395120 & -0.164920 & -6.126810 \\ \text { F } & -6.182370 & 2.460390 & -6.094600 \\ \text { F } & -5.130880 & 4.185950 & -4.252360 \\ \text { F } & -3.294950 & 3.302250 & -2.454020 \\ \text { C } & -3.925960 & -0.231340 & 4.298080 \\ \text { C } & -4.876940 & -0.677700 & 5.230750 \\ \text { C } & -5.284020 & -2.023030 & 5.213200 \\ \text { C } & -4.744150 & -2.908990 & 4.265610 \\ \text { C } & -3.795070 & -2.439320 & 3.342850 \\ \text { F } & -3.559020 & 1.054240 & 4.333320 \\ \text { F } & -5.395120 & 0.164920 & 6.126810 \\ \text { F } & -6.182370 & -2.460390 & 6.094600 \\ \text { F } & -5.130880 & -4.185950 & 4.252360 \\ \text { F } & -3.294950 & -3.302250 & 2.454020\end{array}$




\section{I.6.2 5-Phenylazoimidazole $\cdot$ NiTPPF $_{20}$}

\begin{tabular}{|c|c|c|c|c|c|c|c|}
\hline \multicolumn{4}{|c|}{ cis- $\alpha$} & \multicolumn{4}{|c|}{ cis- $\beta$} \\
\hline \multicolumn{4}{|c|}{$E_{P B E / S V P}=-5963.873770674$ Hartree } & \multicolumn{4}{|c|}{$E_{P B E / S V P}=-5963.871248498$ Hartree } \\
\hline \multicolumn{4}{|c|}{ NImag $=0$} & \multicolumn{4}{|c|}{ NImag $=0$} \\
\hline C & 2.635940 & -2.589100 & 3.550880 & $\mathrm{C}$ & 0.105230 & -0.163330 & 5.856110 \\
\hline C & 2.524650 & -3.991500 & 3.433470 & $\mathrm{C}$ & -0.637950 & 0.959990 & 6.284140 \\
\hline C & 3.406980 & -4.696020 & 2.603330 & $\mathrm{C}$ & -1.920760 & 0.782700 & 6.824390 \\
\hline C & 4.442800 & -4.021010 & 1.934840 & $\mathrm{C}$ & -2.468520 & -0.505190 & 6.959120 \\
\hline C & 4.581110 & -2.629390 & 2.085750 & $\mathrm{C}$ & -1.719510 & -1.622910 & 6.551910 \\
\hline C & 3.676600 & -1.908310 & 2.875290 & $\mathrm{C}$ & -0.437720 & -1.461130 & 6.003470 \\
\hline $\mathrm{N}$ & 1.803890 & -1.923640 & 4.489570 & $\mathrm{~N}$ & 1.445440 & 0.005010 & 5.401660 \\
\hline $\mathrm{N}$ & 1.081080 & -0.929250 & 4.208350 & $\mathrm{~N}$ & 1.767350 & 0.057290 & 4.181600 \\
\hline C & 0.823680 & -0.452850 & 2.923100 & $\mathrm{C}$ & 0.888250 & 0.002370 & 3.113580 \\
\hline C & 0.887110 & -0.867120 & 1.581100 & $\mathrm{C}$ & 1.291260 & 0.072150 & 1.774470 \\
\hline $\mathrm{N}$ & 0.131030 & 0.755790 & 2.879860 & $\mathrm{~N}$ & -0.512800 & -0.099880 & 3.054210 \\
\hline $\mathrm{N}$ & 0.264080 & 0.061740 & 0.792940 & $\mathrm{~N}$ & 0.200130 & 0.012800 & 0.958440 \\
\hline C & -0.174370 & 1.040370 & 1.597830 & $\mathrm{C}$ & -0.874700 & -0.089810 & 1.752700 \\
\hline $\mathrm{H}$ & 1.726940 & -4.510470 & 3.987420 & $\mathrm{H}$ & -0.195700 & 1.964570 & 6.189910 \\
\hline $\mathrm{H}$ & 3.280470 & -5.783900 & 2.478610 & $\mathrm{H}$ & -2.497370 & 1.664690 & 7.148900 \\
\hline $\mathrm{H}$ & 5.150470 & -4.580970 & 1.302330 & $\mathrm{H}$ & -3.473370 & -0.638040 & 7.390770 \\
\hline $\mathrm{H}$ & 5.398720 & -2.089540 & 1.582060 & $\mathrm{H}$ & -2.135360 & -2.637880 & 6.664280 \\
\hline $\mathrm{H}$ & 3.786800 & -0.817960 & 2.988400 & $\mathrm{H}$ & 0.158930 & -2.333770 & 5.692700 \\
\hline $\mathrm{H}$ & 1.326010 & -1.777810 & 1.159950 & $\mathrm{H}$ & 2.317500 & 0.166960 & 1.398650 \\
\hline $\mathrm{H}$ & -0.681170 & 1.956350 & 1.268350 & $\mathrm{H}$ & -1.915210 & -0.162090 & 1.405530 \\
\hline $\mathrm{H}$ & -0.095070 & 1.299170 & 3.718020 & $\mathrm{H}$ & -1.136390 & -0.181130 & 3.863030 \\
\hline C & 1.360320 & 2.901510 & -1.348620 & $\mathrm{C}$ & 1.411800 & 2.860110 & -1.318930 \\
\hline $\mathrm{N}$ & 1.538650 & 1.543740 & -1.481940 & $\mathrm{~N}$ & 1.600200 & 1.496020 & -1.319180 \\
\hline C & 2.645450 & 3.581030 & -1.334160 & $\mathrm{C}$ & 2.691640 & 3.546540 & -1.360850 \\
\hline C & 3.602130 & 2.610980 & -1.480550 & $\mathrm{C}$ & 3.656850 & 2.575030 & -1.389450 \\
\hline C & 2.897240 & 1.343420 & -1.564270 & $\mathrm{C}$ & 2.962370 & 1.299430 & -1.356310 \\
\hline C & 0.110110 & 3.554650 & -1.264530 & $\mathrm{C}$ & 0.161900 & 3.516260 & -1.284630 \\
\hline C & -1.146440 & 2.907540 & -1.315460 & $\mathrm{C}$ & -1.092070 & 2.866970 & -1.259070 \\
\hline C & -2.421330 & 3.603540 & -1.321900 & $\mathrm{C}$ & -2.367770 & 3.562030 & -1.254870 \\
\hline C & -3.394990 & 2.639100 & -1.348900 & $\mathrm{C}$ & -3.340320 & 2.597420 & -1.222450 \\
\hline C & -2.711740 & 1.358090 & -1.363510 & $\mathrm{C}$ & -2.653740 & 1.317280 & -1.225910 \\
\hline $\mathrm{N}$ & -1.346270 & 1.544780 & -1.354240 & $\mathrm{~N}$ & -1.289660 & 1.504510 & -1.249160 \\
\hline $\mathrm{Ni}$ & 0.099910 & 0.087640 & -1.244480 & $\mathrm{Ni}$ & 0.159470 & 0.052890 & -1.078010 \\
\hline $\mathrm{N}$ & 1.512900 & -1.343550 & -1.647880 & $\mathrm{~N}$ & 1.593360 & -1.392840 & -1.355040 \\
\hline C & 1.313180 & -2.704330 & -1.704130 & $\mathrm{C}$ & 1.395010 & -2.754810 & -1.382030 \\
\hline C & 2.574030 & -3.391850 & -1.920570 & $\mathrm{C}$ & 2.669450 & -3.450060 & -1.441290 \\
\hline C & 3.542570 & -2.424000 & -1.978980 & $\mathrm{C}$ & 3.642460 & -2.486010 & -1.431680 \\
\hline C & 2.868520 & -1.150610 & -1.796230 & $\mathrm{C}$ & 2.956830 & -1.205850 & -1.383260 \\
\hline C & -3.370650 & 0.108870 & -1.359680 & $\mathrm{C}$ & -3.307560 & 0.065460 & -1.212420 \\
\hline C & -2.724790 & -1.146490 & -1.383740 & $\mathrm{C}$ & -2.659920 & -1.189240 & -1.263140 \\
\hline C & -3.421500 & -2.422420 & -1.377110 & $\mathrm{C}$ & -3.355190 & -2.463990 & -1.300410 \\
\hline C & -2.457480 & -3.394970 & -1.413980 & $\mathrm{C}$ & -2.388860 & -3.435050 & -1.342360 \\
\hline C & -1.178760 & -2.707320 & -1.466570 & $\mathrm{C}$ & -1.108920 & -2.748630 & -1.336420 \\
\hline $\mathrm{N}$ & -1.364420 & -1.343880 & -1.440660 & $\mathrm{~N}$ & -1.297240 & -1.385170 & -1.292000 \\
\hline C & 0.068230 & -3.358750 & -1.570950 & $\mathrm{C}$ & 0.141450 & -3.403790 & -1.369430 \\
\hline C & 3.530760 & 0.094390 & -1.734250 & $\mathrm{C}$ & 3.611750 & 0.045270 & -1.369990 \\
\hline $\mathrm{H}$ & -4.481920 & 2.785170 & -1.361140 & $\mathrm{H}$ & -4.427450 & 2.742170 & -1.209540 \\
\hline $\mathrm{H}$ & -2.556710 & 4.692010 & -1.323720 & $\mathrm{H}$ & -2.503930 & 4.649960 & -1.284460 \\
\hline $\mathrm{H}$ & -4.509810 & -2.557430 & -1.358000 & $\mathrm{H}$ & -4.443730 & -2.599370 & -1.309600 \\
\hline $\mathrm{H}$ & -2.600420 & -4.482480 & -1.422560 & $\mathrm{H}$ & -2.531980 & -4.521840 & -1.379270 \\
\hline $\mathrm{H}$ & 4.619510 & -2.561650 & -2.134810 & $\mathrm{H}$ & 4.729200 & -2.630170 & -1.461720 \\
\hline $\mathrm{H}$ & 2.700650 & -4.476070 & -2.028220 & $\mathrm{H}$ & 2.803390 & -4.537460 & -1.492520 \\
\hline $\mathrm{H}$ & 2.798650 & 4.662460 & -1.234780 & $\mathrm{H}$ & 2.835490 & 4.633730 & -1.369680 \\
\hline
\end{tabular}




\begin{tabular}{|c|c|c|c|c|c|c|c|}
\hline H & 4.690060 & 2.741950 & -1.534100 & $\mathrm{H}$ & 4.744080 & 2.710390 & -1.440740 \\
\hline c & 0.101240 & 5.037410 & -1.085940 & C & 0.163620 & 5.010920 & -1.275560 \\
\hline c & 5.021210 & 0.073620 & -1.851670 & C & 5.105740 & 0.045150 & -1.348380 \\
\hline C & -4.865370 & 0.115810 & -1.329710 & C & -4.799020 & 0.063770 & -1.120620 \\
\hline C & 0.100470 & -4.850670 & -1.490150 & C & 0.139850 & -4.898670 & -1.389720 \\
\hline C & 0.570690 & -5.474110 & -0.318560 & C & 0.504680 & -5.641840 & -0.250720 \\
\hline C & 0.679360 & -6.868620 & -0.207170 & C & 0.510790 & -7.046770 & -0.253710 \\
\hline C & 0.288050 & -7.676800 & -1.288460 & C & 0.146440 & -7.736700 & -1.422480 \\
\hline C & -0.199480 & -7.083920 & -2.466460 & C & -0.221870 & -7.021650 & -2.574770 \\
\hline C & -0.291960 & -5.683590 & -2.554160 & C & -0.221770 & -5.616430 & -2.546060 \\
\hline $\mathrm{F}$ & 0.940230 & -4.727010 & 0.730240 & $\mathrm{~F}$ & 0.855050 & -5.008730 & 0.875380 \\
\hline $\mathrm{F}$ & 1.147490 & -7.426490 & 0.914800 & $\mathrm{~F}$ & 0.854510 & -7.730210 & 0.841040 \\
\hline $\mathrm{F}$ & 0.381550 & -9.003410 & -1.198910 & $\mathrm{~F}$ & 0.149230 & -9.069820 & -1.437490 \\
\hline $\mathrm{F}$ & -0.565420 & -7.852540 & -3.494550 & $\mathrm{~F}$ & -0.563480 & -7.681670 & -3.683900 \\
\hline $\mathrm{F}$ & -0.753300 & -5.150790 & -3.689630 & $\mathrm{~F}$ & -0.575740 & -4.959320 & -3.655150 \\
\hline C & -5.574350 & -0.236390 & -0.165270 & C & -5.444890 & -0.295610 & 0.077200 \\
\hline C & -6.978850 & -0.238490 & -0.124770 & C & -6.843970 & -0.310900 & 0.194490 \\
\hline C & -7.703180 & 0.120960 & -1.274050 & C & -7.630140 & 0.042840 & -0.915880 \\
\hline C & -7.022610 & 0.478030 & -2.450570 & C & -7.014290 & 0.409240 & -2.125050 \\
\hline C & -5.617350 & 0.472550 & -2.465770 & C & -5.611650 & 0.416540 & -2.214810 \\
\hline $\mathrm{F}$ & -4.907380 & -0.580900 & 0.942790 & $\mathrm{~F}$ & -4.716410 & -0.630660 & 1.155130 \\
\hline $\mathrm{F}$ & -7.629330 & -0.572790 & 0.992780 & $\mathrm{~F}$ & -7.430140 & -0.651460 & 1.345660 \\
\hline $\mathrm{F}$ & -9.036070 & 0.123390 & -1.248080 & $\mathrm{~F}$ & -8.959610 & 0.033230 & -0.820580 \\
\hline $\mathrm{F}$ & -7.714820 & 0.815560 & -3.540930 & $\mathrm{~F}$ & -7.763090 & 0.743100 & -3.177690 \\
\hline $\mathrm{F}$ & -4.994220 & 0.816610 & -3.597690 & $\mathrm{~F}$ & -5.053860 & 0.770240 & -3.376530 \\
\hline C & 5.845370 & 0.159990 & -0.715210 & C & 5.806790 & 0.392790 & -0.177700 \\
\hline C & 7.244110 & 0.074750 & -0.802710 & C & 7.210590 & 0.400470 & -0.127910 \\
\hline C & 7.847850 & -0.078650 & -2.062400 & C & 7.941610 & 0.053080 & -1.277170 \\
\hline C & 7.050740 & -0.155410 & -3.217550 & C & 7.269070 & -0.300020 & -2.459600 \\
\hline C & 5.652210 & -0.080760 & -3.100520 & C & 5.863890 & -0.299490 & -2.483450 \\
\hline $\mathrm{F}$ & 5.297680 & 0.296210 & 0.502320 & $\mathrm{~F}$ & 5.130070 & 0.721910 & 0.931330 \\
\hline $\mathrm{F}$ & 7.997720 & 0.122770 & 0.299080 & $\mathrm{~F}$ & 7.854200 & 0.728970 & 0.994480 \\
\hline $\mathrm{F}$ & 9.174840 & -0.156470 & -2.161040 & $\mathrm{~F}$ & 9.274240 & 0.056210 & -1.244560 \\
\hline $\mathrm{F}$ & 7.625150 & -0.301020 & -4.413400 & $\mathrm{~F}$ & 7.968670 & -0.627740 & -3.548120 \\
\hline $\mathrm{F}$ & 4.917690 & -0.167220 & -4.213250 & $\mathrm{~F}$ & 5.247900 & -0.637700 & -3.621030 \\
\hline C & 0.499790 & 5.919120 & -2.109720 & C & 0.515990 & 5.751390 & -2.420790 \\
\hline C & 0.465290 & 7.313960 & -1.941220 & C & 0.517960 & 7.156770 & -2.421830 \\
\hline C & 0.024670 & 7.854700 & -0.721040 & C & 0.159550 & 7.850060 & -1.253270 \\
\hline C & -0.377090 & 7.000000 & 0.320200 & C & -0.197270 & 7.137900 & -0.095640 \\
\hline C & -0.328360 & 5.610580 & 0.127110 & C & -0.191220 & 5.733190 & -0.119650 \\
\hline $\mathrm{F}$ & 0.917700 & 5.437850 & -3.284290 & $\mathrm{~F}$ & 0.858470 & 5.116890 & -3.546790 \\
\hline $\mathrm{F}$ & 0.841620 & 8.125460 & -2.931720 & $\mathrm{~F}$ & 0.851840 & 7.837330 & -3.520670 \\
\hline $\mathrm{F}$ & -0.013710 & 9.176080 & -0.551260 & $\mathrm{~F}$ & 0.157670 & 9.183160 & -1.242790 \\
\hline $\mathrm{F}$ & -0.794040 & 7.510940 & 1.481380 & $\mathrm{~F}$ & -0.533400 & 7.800320 & 1.014440 \\
\hline $\mathrm{F}$ & -0.708650 & 4.822110 & 1.146140 & $\mathrm{~F}$ & -0.533670 & 5.079370 & 0.997170 \\
\hline
\end{tabular}




\section{trans- $\alpha$}

$E_{P B E / S V P}=-5963.893533912$ Hartree

$\mathrm{NImag}=0$

\begin{tabular}{|c|c|c|c|}
\hline C & -3.078050 & -0.335400 & 5.183830 \\
\hline C & -4.468830 & -0.464180 & 4.955840 \\
\hline C & -5.350110 & -0.573550 & 6.039240 \\
\hline C & -4.851750 & -0.555670 & 7.353380 \\
\hline C & -3.466350 & -0.426440 & 7.584160 \\
\hline C & -2.579310 & -0.316710 & 6.510550 \\
\hline$N$ & -2.288580 & -0.238000 & 4.025990 \\
\hline$N$ & -1.031350 & -0.121510 & 4.211640 \\
\hline $\mathrm{C}$ & -0.314990 & -0.039610 & 3.0387 \\
\hline $\mathrm{C}$ & -0.640360 & -0.060580 & 1.678920 \\
\hline $\mathrm{N}$ & 1.068640 & 0.091050 & 3.077740 \\
\hline$N$ & 0.504550 & 0.051440 & 0.942060 \\
\hline $\mathrm{C}$ & 1.527540 & 0.142790 & 1.802910 \\
\hline $\mathrm{H}$ & -4.823010 & -0.475320 & 3.912 \\
\hline $\mathrm{H}$ & -6.432940 & -0.674450 & 5.859670 \\
\hline $\mathrm{H}$ & -5.544270 & -0.643020 & 8.206790 \\
\hline $\mathrm{H}$ & -3.082440 & -0.412910 & 8.617890 \\
\hline $\mathrm{H}$ & -1.494020 & -0.216080 & 6.663220 \\
\hline $\mathrm{H}$ & -1.636330 & -0.153550 & 1.233370 \\
\hline $\mathrm{H}$ & 2.585920 & 0.246810 & 1.528790 \\
\hline $\mathrm{H}$ & 1.618830 & 0.136880 & 3.939050 \\
\hline $\mathrm{C}$ & 1.825380 & 2.862410 & -1.327860 \\
\hline $\mathrm{N}$ & 2.015660 & 1.499110 & -1.295950 \\
\hline C & 3.104700 & 3.550510 & -1.331690 \\
\hline C & 4.072170 & 2.580120 & -1.302400 \\
\hline $\mathrm{C}$ & 3.378150 & 1.304200 & -1.275630 \\
\hline C & 0.573770 & 3.515990 & -1.350760 \\
\hline C & -0.679100 & 2.865320 & -1.355870 \\
\hline C & -1.955400 & 3.559400 & -1.384070 \\
\hline C & -2.926870 & 2.594270 & -1.349850 \\
\hline C & -2.238550 & 1.314760 & -1.32 \\
\hline $\mathrm{N}$ & -0.875190 & 1.503130 & -1.333130 \\
\hline $\mathrm{Ni}$ & 0.567550 & 0.056450 & -1.090140 \\
\hline $\mathrm{N}$ & 2.010790 & -1.391130 & -1.300670 \\
\hline $\mathrm{C}$ & 1.814100 & -2.753080 & -1.334940 \\
\hline C & 3.090620 & -3.446830 & -1.353020 \\
\hline $\mathrm{C}$ & 4.062350 & -2.482000 & -1.308490 \\
\hline C & 3.374390 & -1.202600 & -1.282250 \\
\hline C & -2.891390 & 0.062480 & -1.290340 \\
\hline C & -2.242480 & -1.190890 & -1.323340 \\
\hline C & -2.937640 & -2.466120 & -1.346620 \\
\hline C & -1.971030 & -3.436890 & -1.370760 \\
\hline $\mathrm{C}$ & -0.690770 & -2.749130 & -1.362050 \\
\hline $\mathrm{N}$ & -0.879810 & -1.385800 & -1.340840 \\
\hline $\mathrm{C}$ & 0.560640 & -3.403580 & -1.357930 \\
\hline $\mathrm{C}$ & 4.026770 & 0.049580 & -1.249130 \\
\hline $\mathrm{H}$ & -4.014120 & 2.737800 & -1.352770 \\
\hline $\mathrm{H}$ & -2.091910 & 4.646750 & -1.429650 \\
\hline $\mathrm{H}$ & -4.026450 & -2.600040 & -1.356420 \\
\hline $\mathrm{H}$ & -2.113540 & -4.524190 & -1.390240 \\
\hline $\mathrm{H}$ & 5.149620 & -2.626050 & -1.30453 \\
\hline $\mathrm{H}$ & 3.227710 & -4.533840 & -1.404440 \\
\hline $\mathrm{H}$ & 3.246450 & 4.637820 & -1.358380 \\
\hline $\mathrm{H}$ & 5.160590 & 2.716660 & -1.31371 \\
\hline $\mathrm{C}$ & 0.573530 & 5.011000 & -1.3606 \\
\hline
\end{tabular}

trans- $\beta$

$E_{P B E / S V P}=-5963.895992443$ Hartree NImag $=0$

\begin{tabular}{|c|c|c|c|}
\hline C & -1.153990 & -0.161760 & 6.377810 \\
\hline C & -0.367390 & -0.119130 & 7.551560 \\
\hline C & -0.975260 & -0.189470 & 8.811530 \\
\hline C & -2.372670 & -0.302020 & 8.908590 \\
\hline C & -3.161220 & -0.344390 & 7.740380 \\
\hline C & -2.562870 & -0.275460 & 6.479740 \\
\hline$N$ & -0.448410 & -0.084880 & 5.161710 \\
\hline $\mathrm{N}$ & -1.165360 & -0.132900 & 4.104680 \\
\hline C & -0.433740 & -0.053790 & 2.945810 \\
\hline C & -0.863990 & -0.080650 & 1.616080 \\
\hline$N$ & 0.950060 & 0.073280 & 2.881170 \\
\hline$N$ & 0.225090 & 0.025900 & 0.792720 \\
\hline C & 1.307300 & 0.117850 & 1.577940 \\
\hline $\mathrm{H}$ & 0.724970 & -0.030340 & 7.440000 \\
\hline $\mathrm{H}$ & -0.357990 & -0.156060 & 9.723940 \\
\hline $\mathrm{H}$ & -2.854110 & -0.356970 & 9.898880 \\
\hline $\mathrm{H}$ & -4.257320 & -0.432480 & 7.822590 \\
\hline $\mathrm{H}$ & -3.155870 & -0.307040 & 5.553380 \\
\hline $\mathrm{H}$ & -1.889460 & -0.173660 & 1.238910 \\
\hline $\mathrm{H}$ & 2.342340 & 0.218210 & 1.222850 \\
\hline $\mathrm{H}$ & 1.533960 & 0.118170 & 3.722150 \\
\hline C & 1.507980 & 2.840180 & -1.468710 \\
\hline $\mathrm{N}$ & 1.695890 & 1.476180 & -1.450240 \\
\hline C & 2.788370 & 3.526180 & -1.475770 \\
\hline C & 3.754400 & 2.554130 & -1.462270 \\
\hline C & 3.058560 & 1.279220 & -1.440500 \\
\hline C & 0.257600 & 3.496240 & -1.479380 \\
\hline C & -0.996340 & 2.847580 & -1.491390 \\
\hline C & -2.271040 & 3.543880 & -1.533100 \\
\hline C & -3.244200 & 2.579880 & -1.531490 \\
\hline C & -2.558370 & 1.299150 & -1.505220 \\
\hline $\mathrm{N}$ & -1.194830 & 1.485530 & -1.482230 \\
\hline $\mathrm{Ni}$ & 0.241370 & 0.034950 & -1.242700 \\
\hline $\mathrm{N}$ & 1.687040 & -1.414010 & -1.458890 \\
\hline C & 1.488760 & -2.775980 & -1.484360 \\
\hline C & 2.764050 & -3.471740 & -1.502470 \\
\hline C & 3.737260 & -2.508010 & -1.468080 \\
\hline C & 3.051110 & -1.227590 & -1.446990 \\
\hline C & -3.213520 & 0.048210 & -1.506680 \\
\hline C & -2.565360 & -1.206560 & -1.518640 \\
\hline C & -3.260940 & -2.481220 & -1.565300 \\
\hline C & -2.295810 & -3.453460 & -1.560000 \\
\hline C & -1.015450 & -2.767930 & -1.518950 \\
\hline $\mathrm{N}$ & -1.203250 & -1.404100 & -1.496870 \\
\hline C & 0.234520 & -3.424570 & -1.505530 \\
\hline $\mathrm{C}$ & 3.705760 & 0.023680 & -1.420310 \\
\hline $\mathrm{H}$ & -4.331120 & 2.724550 & -1.554030 \\
\hline $\mathrm{H}$ & -2.405010 & 4.631910 & -1.568720 \\
\hline $\mathrm{H}$ & -4.348700 & -2.615330 & -1.609420 \\
\hline $\mathrm{H}$ & -2.439790 & -4.540410 & -1.584740 \\
\hline $\mathrm{H}$ & 4.824340 & -2.653460 & -1.467670 \\
\hline $\mathrm{H}$ & 2.899250 & -4.559280 & -1.546960 \\
\hline $\mathrm{H}$ & 2.931940 & 4.613400 & -1.493370 \\
\hline $\mathrm{H}$ & 4.842900 & 2.689020 & -1.480610 \\
\hline C & 0.259680 & 4.991180 & -1.479960 \\
\hline
\end{tabular}




\begin{tabular}{|c|c|c|c|c|c|c|c|}
\hline C & 5.518520 & 0.050440 & -1.165410 & $\mathrm{C}$ & 5.198040 & 0.021450 & -1.345290 \\
\hline C & -4.381000 & 0.069810 & -1.162530 & $\mathrm{C}$ & -4.707440 & 0.048500 & -1.474320 \\
\hline C & 0.560810 & -4.898170 & -1.367660 & $\mathrm{C}$ & 0.233030 & -4.919280 & -1.514560 \\
\hline C & 0.927280 & -5.634350 & -0.224210 & $\mathrm{C}$ & 0.592200 & -5.654840 & -0.368460 \\
\hline C & 0.933620 & -7.039260 & -0.217820 & $C$ & 0.599190 & -7.059780 & -0.361600 \\
\hline C & 0.567800 & -7.737190 & -1.381250 & $\mathrm{C}$ & 0.240010 & -7.758260 & -1.526790 \\
\hline C & 0.197970 & -7.029800 & -2.537670 & $\mathrm{C}$ & -0.122400 & -7.051410 & -2.685900 \\
\hline C & 0.198280 & -5.624490 & -2.518690 & $\mathrm{C}$ & -0.122000 & -5.646110 & -2.667580 \\
\hline $\mathrm{F}$ & 1.278920 & -4.994720 & 0.897670 & $\mathrm{~F}$ & 0.937690 & -5.014440 & 0.754850 \\
\hline $\mathrm{F}$ & 1.279070 & -7.715430 & 0.881090 & $\mathrm{~F}$ & 0.938840 & -7.735410 & 0.739360 \\
\hline $\mathrm{F}$ & 0.571070 & -9.070560 & -1.387420 & $F$ & 0.243290 & -9.091570 & -1.532360 \\
\hline $\mathrm{F}$ & -0.143920 & -7.697330 & -3.642380 & $\mathrm{~F}$ & -0.458310 & -7.719330 & -3.792160 \\
\hline $\mathrm{F}$ & -0.155500 & -4.975930 & -3.633050 & $\mathrm{~F}$ & -0.468620 & -4.998060 & -3.784550 \\
\hline C & -4.994020 & -0.208660 & 0.074050 & $C$ & -5.400690 & -0.304800 & -0.300940 \\
\hline C & -6.386590 & -0.158630 & 0.242740 & $C$ & -6.804000 & -0.313520 & -0.241490 \\
\hline C & -7.202360 & 0.163680 & -0.855390 & $\mathrm{C}$ & -7.543250 & 0.039160 & -1.383710 \\
\hline C & -6.619810 & 0.437700 & -2.105190 & $\mathrm{C}$ & -6.878740 & 0.397630 & -2.569020 \\
\hline C & -5.222080 & 0.387530 & -2.245210 & $\mathrm{C}$ & -5.473650 & 0.398700 & -2.602210 \\
\hline $\mathrm{F}$ & -4.242220 & -0.514800 & 1.141570 & $\mathrm{~F}$ & -4.717080 & -0.639750 & 0.802790 \\
\hline $\mathrm{F}$ & -6.936070 & -0.406160 & 1.436860 & $\mathrm{~F}$ & -7.439590 & -0.647330 & 0.884750 \\
\hline $\mathrm{F}$ & -8.526900 & 0.213440 & -0.711400 & $\mathrm{~F}$ & -8.875810 & 0.035230 & -1.341800 \\
\hline $\mathrm{F}$ & -7.395110 & 0.743940 & -3.147700 & $\mathrm{~F}$ & -7.585680 & 0.729750 & -3.651420 \\
\hline $\mathrm{F}$ & -4.695510 & 0.659840 & -3.443140 & $\mathrm{~F}$ & -4.866190 & 0.743270 & -3.742180 \\
\hline C & 6.170690 & 0.386330 & 0.035880 & $\mathrm{C}$ & 5.858280 & 0.358920 & -0.148910 \\
\hline C & 7.570510 & 0.393140 & 0.146750 & $\mathrm{C}$ & 7.258590 & 0.360630 & -0.045220 \\
\hline C & 8.350160 & 0.052180 & -0.972140 & $\mathrm{C}$ & 8.030880 & 0.016550 & -1.168230 \\
\hline C & 7.727650 & -0.288520 & -2.185330 & $\mathrm{C}$ & 7.400470 & -0.326190 & -2.376750 \\
\hline C & 6.324700 & -0.287040 & -2.269070 & $\mathrm{C}$ & 5.997040 & -0.319800 & -2.453040 \\
\hline $\mathrm{F}$ & 5.448450 & 0.708550 & 1.121370 & $\mathrm{~F}$ & 5.142880 & 0.684040 & 0.940530 \\
\hline $\mathrm{F}$ & 8.163890 & 0.710410 & 1.300940 & $\mathrm{~F}$ & 7.859310 & 0.678330 & 1.104920 \\
\hline $\mathrm{F}$ & 9.680200 & 0.052190 & -0.881980 & $\mathrm{~F}$ & 9.361330 & 0.012900 & -1.085620 \\
\hline $\mathrm{F}$ & 8.470700 & -0.610220 & -3.246060 & $F$ & 8.136870 & -0.651560 & -3.440910 \\
\hline $\mathrm{F}$ & 5.759750 & -0.616040 & -3.434790 & $\mathrm{~F}$ & 5.424450 & -0.650120 & -3.614590 \\
\hline C & 0.930510 & 5.736730 & -2.513500 & $\mathrm{C}$ & 0.621280 & 5.723540 & -2.627220 \\
\hline C & 0.930060 & 7.142090 & -2.532780 & $C$ & 0.621440 & 7.128930 & -2.638210 \\
\hline C & 0.564150 & 7.849360 & -1.375000 & $\mathrm{C}$ & 0.253830 & 7.829600 & -1.476950 \\
\hline C & 0.204060 & 7.151590 & -0.209660 & $\mathrm{C}$ & -0.110880 & 7.125210 & -0.317030 \\
\hline C & 0.211610 & 5.746590 & -0.215630 & $\mathrm{C}$ & -0.104690 & 5.720230 & -0.331510 \\
\hline $\mathrm{F}$ & 1.281580 & 5.087470 & -3.628280 & $\mathrm{~F}$ & 0.974510 & 5.080860 & -3.745210 \\
\hline $\mathrm{F}$ & 1.267940 & 7.809710 & -3.638690 & $\mathrm{~F}$ & 0.962730 & 7.802760 & -3.739220 \\
\hline $\mathrm{F}$ & 0.560020 & 9.182750 & -1.381460 & $\mathrm{~F}$ & 0.251220 & 9.162960 & -1.475430 \\
\hline $\mathrm{F}$ & -0.137660 & 7.827940 & 0.890270 & $\mathrm{~F}$ & -0.454420 & 7.795310 & 0.786110 \\
\hline $\mathrm{F}$ & -0.134710 & 5.106290 & 0.907220 & $\mathrm{~F}$ & -0.455100 & 5.073290 & 0.786220 \\
\hline
\end{tabular}


I.6.3 1-Methyl-5-phenylazoimidazole $\cdot$ NiTPPF $_{20}$ (2)

cis- $\alpha$

$E_{P B E / S V P}=-6003.099149714$ Hartree

$\mathrm{NImag}=0$

\begin{tabular}{|c|c|c|c|}
\hline C & 2.066860 & -2.320350 & 3.993330 \\
\hline C & 1.967540 & -3.727410 & 3.927060 \\
\hline C & 2.853570 & -4.455910 & 3.122110 \\
\hline C & 3.883760 & -3.798100 & 2.428430 \\
\hline C & 4.012820 & -2.400740 & 2.529600 \\
\hline C & 3.104060 & -1.658230 & 3.293090 \\
\hline$N$ & 1.226880 & -1.636270 & 4.910680 \\
\hline $\mathrm{N}$ & 0.550060 & -0.611830 & 4.617520 \\
\hline $\mathrm{C}$ & 0.324490 & -0.126050 & 3.334540 \\
\hline C & 0.300980 & -0.611480 & 2.014390 \\
\hline$N$ & -0.257210 & 1.147620 & 3.263960 \\
\hline$N$ & -0.260260 & 0.329750 & 1.200640 \\
\hline $\mathrm{C}$ & -0.572060 & 1.381660 & 1.971950 \\
\hline $\mathrm{H}$ & 1.174470 & -4.230430 & 4.502120 \\
\hline $\mathrm{H}$ & 2.735960 & -5.548620 & 3.037470 \\
\hline $\mathrm{H}$ & 4.594830 & -4.375220 & 1.815 \\
\hline $\mathrm{H}$ & 4.826550 & -1.873990 & 2.006140 \\
\hline $\mathrm{H}$ & 3.207980 & -0.563860 & 3.365150 \\
\hline $\mathrm{H}$ & 0.635270 & -1.582940 & 1.633490 \\
\hline $\mathrm{H}$ & -1.003880 & 2.325530 & 1.614530 \\
\hline C & 0.805800 & 3.158480 & -0.898590 \\
\hline$N$ & 0.990380 & 1.805960 & -1.070970 \\
\hline$C$ & 2.088250 & 3.842990 & -0.865940 \\
\hline $\mathrm{C}$ & 3.048580 & 2.882910 & -1.049280 \\
\hline $\mathrm{C}$ & 2.349720 & 1.614430 & -1.161160 \\
\hline C & -0.449960 & 3.800510 & -0.795220 \\
\hline C & -1.701520 & 3.147330 & -0.885540 \\
\hline C & -2.980170 & 3.836180 & -0.904650 \\
\hline C & -3.946310 & 2.866990 & -0.980910 \\
\hline C & -3.254340 & 1.590340 & -1.002830 \\
\hline$N$ & -1.891110 & 1.785040 & -0.959470 \\
\hline $\mathrm{Ni}$ & -0.437900 & 0.336360 & -0.834550 \\
\hline$N$ & 0.988390 & -1.085260 & -1.218040 \\
\hline $\mathrm{C}$ & 0.801670 & -2.448190 & -1.249680 \\
\hline $\mathrm{C}$ & 2.069850 & -3.127920 & -1.449600 \\
\hline $\mathrm{C}$ & 3.028380 & -2.151630 & -1.530220 \\
\hline $\mathrm{C}$ & 2.341640 & -0.881920 & -1.369700 \\
\hline C & -3.905000 & 0.337050 & -1.024940 \\
\hline C & -3.251010 & -0.914490 & -1.028230 \\
\hline C & -3.939870 & -2.194720 & -1.032070 \\
\hline C & -2.969460 & -3.161560 & -1.028810 \\
\hline $\mathrm{C}$ & -1.693800 & -2.466550 & -1.052440 \\
\hline$N$ & -1.888560 & -1.104160 & -1.043700 \\
\hline C & -0.440130 & -3.110240 & -1.121610 \\
\hline $\mathrm{C}$ & 2.991580 & 0.370260 & -1.332660 \\
\hline $\mathrm{H}$ & -5.033570 & 3.006490 & -1.01751 \\
\hline $\mathrm{H}$ & -3.121470 & 4.923880 & -0.885610 \\
\hline $\mathrm{H}$ & -5.027410 & -2.336590 & -1.04413 \\
\hline $\mathrm{H}$ & -3.106280 & -4.249830 & -1.03012 \\
\hline $\mathrm{H}$ & 4.106460 & -2.280340 & -1.686510 \\
\hline $\mathrm{H}$ & 2.207070 & -4.212730 & -1.53624 \\
\hline $\mathrm{H}$ & 2.239310 & 4.921010 & -0.733630 \\
\hline $\mathrm{H}$ & 4.135750 & 3.020450 & -1.10248 \\
\hline
\end{tabular}

cis- $\beta$

$E_{P B E / S V P}=-6003.091275671$ Hartree NImag $=0$

C $\quad 0.553210 \quad-1.459150$

$\begin{array}{llll}\text { C } & -0.020030 & -1.737060 & 6.629110\end{array}$

$\begin{array}{llll}\text { C } & -0.609880 & -2.981110 & 6.878670\end{array}$

$\begin{array}{llll}\text { C } & -0.581350 & -3.986560 & 5.894200\end{array}$

$\begin{array}{llll}\text { C } & 0.032500 & -3.734230 & 4.654100\end{array}$

$\begin{array}{llll}C & 0.583300 & -2.475380 & 4.375600\end{array}$

N $\quad 1.244450 \quad-0.230280 \quad 5.238940$

N $\quad 1.345120 \quad 0.445340 \quad 4.175020$

$\begin{array}{llll}\text { C } & 0.578250 & 0.302050 & 3.024040\end{array}$

C $\quad 1.038620 \quad 0.545490 \quad 1.724370$

$\begin{array}{llll}\mathrm{N} & -0.801630 & 0.058560 & 2.889910\end{array}$

$\begin{array}{llll}\mathrm{N} & 0.006960 & 0.401910 & 0.840000\end{array}$

$\begin{array}{llll}\text { C } & -1.085890 & 0.143300 & 1.562000\end{array}$

$\begin{array}{llll}\mathrm{H} & 0.013250 & -0.951190 & 7.400500\end{array}$

$\begin{array}{llll}H & -1.073960 & -3.180030 & 7.858690\end{array}$

$\begin{array}{llll}H & -1.020280 & -4.976230 & 6.100500\end{array}$

$\begin{array}{llll}\mathrm{H} & 0.090260 & -4.526760 & 3.890900\end{array}$

$\begin{array}{llll}\mathrm{H} & 1.070080 & -2.294300 & 3.404360\end{array}$

$\begin{array}{llll}\mathrm{H} & 2.066120 & 0.772580 & 1.415280\end{array}$

$\begin{array}{llll}\text { H } & -2.106110 & 0.052940 & 1.163880\end{array}$

C $\quad 1.306740 \quad 3.174770 \quad-1.640080$

$\mathrm{N} \quad 1.511490 \quad 1.822020 \quad-1.486680$

C $\quad 2.578020 \quad 3.877820 \quad-1.669240$

C $\quad 3.554890 \quad 2.927460 \quad-1.536570$

$\begin{array}{llll}\text { C } & 2.875790 & 1.648060 & -1.423870\end{array}$

$\begin{array}{llll}\text { C } & 0.049470 & 3.808160 & -1.758630\end{array}$

$\begin{array}{llll}\text { C } & -1.196050 & 3.142930 & -1.720450\end{array}$

C $\quad-2.479970 \quad 3.811600 \quad-1.843350$

C $\quad-3.440030 \quad 2.840960 \quad-1.735610$

C $\quad-2.737250 \quad 1.578700 \quad-1.580230$

N $\quad-1.376010 \quad 1.786160 \quad-1.570250$

$\mathrm{Ni} \quad 0.079220 \quad 0.381190 \quad-1.197670$

N $\quad 1.548020 \quad-1.057160 \quad-1.287360$

C $\quad 1.366060 \quad-2.415370 \quad-1.168060$

C $\quad 2.649080 \quad-3.091890 \quad-1.074650$

C $\quad 3.609760 \quad-2.115880 \quad-1.119950$

C $\quad 2.907870 \quad-0.850040 \quad-1.246180$

C $\quad-3.375600 \quad 0.322540 \quad-1.474930$

C $\quad-2.711890 \quad-0.922340 \quad-1.407760$

$\begin{array}{llll}\text { C } & -3.388790 & -2.205620 & -1.337400\end{array}$

C $\quad-2.408660 \quad-3.158990 \quad-1.235720$

C $\quad-1.139520 \quad-2.452650 \quad-1.254010$

N $\quad-1.346990 \quad-1.097260 \quad-1.376030$

C $\quad 0.120190 \quad-3.078260 \quad-1.131380$

C $\quad 3.543000 \quad 0.410000 \quad-1.292300$

H $\quad-4.528180 \quad 2.970230 \quad-1.776610$

H $\quad-2.630600 \quad 4.886740 \quad-1.998320$

H $\quad-4.474590 \quad-2.358750 \quad-1.374040$

$\mathrm{H} \quad-2.535830 \quad-4.246210 \quad-1.164330$

H $\quad 4.698260 \quad-2.242650 \quad-1.074160$

H $\quad 2.796540 \quad-4.176320 \quad-0.997910$

H $\quad 2.708590 \quad 4.961400 \quad-1.775640$

$\begin{array}{llll}\mathrm{H} & 4.641270 & 3.078120 & -1.531430\end{array}$ 


\begin{tabular}{|c|c|c|c|c|c|c|c|}
\hline C & -0.465400 & 5.270980 & -0.536660 & C & 0.040340 & 5.289000 & -1.961940 \\
\hline C & 4.482100 & 0.360910 & -1.456090 & C & 5.030990 & 0.448530 & -1.160210 \\
\hline C & -5.400000 & 0.335410 & -1.031910 & C & -4.867020 & 0.317460 & -1.380290 \\
\hline C & -0.399680 & -4.600990 & -1.027790 & C & 0.139020 & -4.544430 & -0.839480 \\
\hline C & 0.066270 & -5.213740 & 0.151050 & C & 0.388530 & -4.985770 & 0.473260 \\
\hline C & 0.179560 & -6.607010 & 0.273580 & C & 0.406710 & -6.345740 & 0.815450 \\
\hline C & -0.202980 & -7.424970 & -0.803480 & C & 0.177690 & -7.307600 & -0.183030 \\
\hline C & -0.685310 & -6.842990 & -1.988990 & C & -0.067670 & -6.898960 & -1.505630 \\
\hline C & -0.781790 & -5.443850 & -2.087930 & C & -0.091850 & -5.527750 & -1.818730 \\
\hline $\mathrm{F}$ & 0.426540 & -4.457850 & 1.196810 & $\mathrm{~F}$ & 0.605360 & -4.086850 & 1.446240 \\
\hline $\mathrm{F}$ & 0.642150 & -7.154800 & 1.402640 & $\mathrm{~F}$ & 0.627280 & -6.727110 & 2.079440 \\
\hline $\mathrm{F}$ & -0.105730 & -8.750570 & -0.702860 & $\mathrm{~F}$ & 0.193100 & -8.604620 & 0.122990 \\
\hline $\mathrm{F}$ & -1.043390 & -7.620470 & -3.013270 & $\mathrm{~F}$ & -0.276650 & -7.815320 & -2.453000 \\
\hline $\mathrm{F}$ & -1.237830 & -4.921790 & -3.230560 & $\mathrm{~F}$ & -0.341980 & -5.171180 & -3.081690 \\
\hline C & -6.135590 & -0.024660 & 0.113560 & C & -5.505050 & 0.071720 & -0.149750 \\
\hline C & -7.540640 & -0.031010 & 0.119580 & C & -6.901530 & 0.108790 & -0.012900 \\
\hline C & -8.238240 & 0.330430 & -1.045520 & C & -7.695370 & 0.394480 & -1.137340 \\
\hline C & -7.530780 & 0.694770 & -2.203720 & C & -7.088700 & 0.638260 & -2.381330 \\
\hline C & -6.125500 & 0.694000 & -2.184470 & C & -5.688140 & 0.591830 & -2.490360 \\
\hline $\mathrm{F}$ & -5.495150 & -0.370860 & 1.236460 & $\mathrm{~F}$ & -4.771950 & -0.195370 & 0.946210 \\
\hline $\mathrm{F}$ & -8.217050 & -0.371510 & 1.219610 & $\mathrm{~F}$ & -7.477350 & -0.114560 & 1.171470 \\
\hline $\mathrm{F}$ & -9.571460 & 0.327980 & -1.051750 & $\mathrm{~F}$ & -9.022560 & 0.436920 & -1.022010 \\
\hline $\mathrm{F}$ & -8.198000 & 1.033790 & -3.309370 & $\mathrm{~F}$ & -7.843520 & 0.912300 & -3.446960 \\
\hline $\mathrm{F}$ & -5.475690 & 1.045100 & -3.298980 & $\mathrm{~F}$ & -5.139090 & 0.828100 & -3.685480 \\
\hline C & 5.310590 & 0.406330 & -0.320670 & C & 5.630920 & 0.908720 & 0.027890 \\
\hline C & 6.709210 & 0.331260 & -0.418210 & C & 7.025400 & 0.982000 & 0.176000 \\
\hline C & 7.307450 & 0.229550 & -1.685800 & C & 7.852790 & 0.582010 & -0.887770 \\
\hline C & 6.505390 & 0.191520 & -2.839380 & C & 7.282660 & 0.112790 & -2.083740 \\
\hline C & 5.107350 & 0.262660 & -2.713100 & C & 5.884200 & 0.047990 & -2.205860 \\
\hline $\mathrm{F}$ & 4.768090 & 0.497220 & 0.903640 & $\mathrm{~F}$ & 4.865900 & 1.291170 & 1.059510 \\
\hline $\mathrm{F}$ & 7.468670 & 0.349270 & 0.680370 & $\mathrm{~F}$ & 7.566520 & 1.423480 & 1.313540 \\
\hline $\mathrm{F}$ & 8.634350 & 0.160650 & -1.793210 & $\mathrm{~F}$ & 9.178330 & 0.647410 & -0.761980 \\
\hline $\mathrm{F}$ & 7.074660 & 0.088600 & -4.042230 & $\mathrm{~F}$ & 8.069800 & -0.262210 & -3.094790 \\
\hline $\mathrm{F}$ & 4.367160 & 0.217100 & -3.824070 & $\mathrm{~F}$ & 5.370860 & -0.402190 & -3.355120 \\
\hline C & -0.022110 & 6.209370 & -1.489510 & C & 0.500870 & 5.855720 & -3.167390 \\
\hline C & -0.020320 & 7.589820 & -1.225290 & C & 0.506920 & 7.244100 & -3.380310 \\
\hline C & -0.484580 & 8.059580 & 0.015090 & C & 0.034990 & 8.100870 & -2.371450 \\
\hline C & -0.941180 & 7.148800 & 0.983460 & C & -0.435460 & 7.565040 & -1.160540 \\
\hline C & -0.921810 & 5.775060 & 0.697660 & C & -0.425490 & 6.173110 & -0.969030 \\
\hline $\mathrm{F}$ & 0.414530 & 5.796160 & -2.682980 & $\mathrm{~F}$ & 0.948260 & 5.066700 & -4.149870 \\
\hline $\mathrm{F}$ & 0.413570 & 8.455990 & -2.142960 & $\mathrm{~F}$ & 0.951100 & 7.751650 & -4.532380 \\
\hline $\mathrm{F}$ & -0.490500 & 9.366570 & 0.275190 & $\mathrm{~F}$ & 0.032510 & 9.420390 & -2.562700 \\
\hline $\mathrm{F}$ & -1.378220 & 7.594070 & 2.163980 & $\mathrm{~F}$ & -0.882600 & 8.380560 & -0.201820 \\
\hline $\mathrm{F}$ & -1.348750 & 4.930250 & 1.654300 & $\mathrm{~F}$ & -0.875260 & 5.695390 & 0.196990 \\
\hline C & -0.450720 & 2.052700 & 4.387440 & C & -1.805670 & -0.089660 & 3.935070 \\
\hline $\mathrm{H}$ & -0.953020 & 1.510930 & 5.214030 & $\mathrm{H}$ & -1.847230 & -1.135010 & 4.306050 \\
\hline $\mathrm{H}$ & 0.525860 & 2.428970 & 4.758590 & $\mathrm{H}$ & -1.565600 & 0.582230 & 4.783860 \\
\hline $\mathrm{H}$ & -1.069640 & 2.907590 & 4.049210 & $\mathrm{H}$ & -2.792600 & 0.196290 & $3.5171 \epsilon$ \\
\hline
\end{tabular}




\section{trans- $\alpha$}

$E_{P B E / S V P}=-6003.118228453$ Hartree

NImag $=0$

\begin{tabular}{|c|c|c|c|}
\hline C & -3.102740 & -0.330900 & 5.057310 \\
\hline C & -4.495710 & -0.447650 & 4.835260 \\
\hline C & -5.373610 & -0.555830 & 5.921500 \\
\hline C & -4.870290 & -0.549200 & 7.233840 \\
\hline C & -3.482930 & -0.432810 & 7.459130 \\
\hline C & -2.599350 & -0.323850 & 6.382410 \\
\hline$N$ & -2.318900 & -0.233490 & 3.895760 \\
\hline$N$ & -1.059400 & -0.120410 & 4.073430 \\
\hline $\mathrm{C}$ & -0.348910 & -0.040600 & 2.898220 \\
\hline $\mathrm{C}$ & -0.695060 & -0.068630 & 1.542590 \\
\hline $\mathrm{N}$ & 1.040870 & 0.092940 & 2.930970 \\
\hline$N$ & 0.437090 & 0.041640 & 0.791500 \\
\hline $\mathrm{C}$ & 1.467880 & 0.138550 & 1.643740 \\
\hline $\mathrm{H}$ & -4.854130 & -0.450990 & 3.793 \\
\hline $\mathrm{H}$ & -6.457890 & -0.647330 & 5.745430 \\
\hline $\mathrm{H}$ & -5.560010 & -0.635660 & 8.089600 \\
\hline $\mathrm{H}$ & -3.094480 & -0.428770 & 8.491310 \\
\hline $\mathrm{H}$ & -1.512800 & -0.233230 & 6.532470 \\
\hline $\mathrm{H}$ & -1.697890 & -0.165560 & 1.113150 \\
\hline $\mathrm{H}$ & 2.523260 & 0.244080 & 1.357150 \\
\hline $\mathrm{C}$ & 1.749710 & 2.854070 & -1.474900 \\
\hline$N$ & 1.940370 & 1.491010 & -1.441420 \\
\hline $\mathrm{C}$ & 3.028790 & 3.542740 & -1.469930 \\
\hline C & 3.996410 & 2.572730 & -1.430920 \\
\hline C & 3.302530 & 1.296560 & -1.409440 \\
\hline C & 0.497830 & 3.507100 & -1.502160 \\
\hline C & -0.754930 & 2.856010 & -1.505410 \\
\hline C & -2.031650 & 3.549550 & -1.533000 \\
\hline C & -3.002660 & 2.584150 & -1.493460 \\
\hline C & -2.313680 & 1.304980 & -1.465270 \\
\hline$N$ & -0.950520 & 1.493860 & -1.478 \\
\hline $\mathrm{Ni}$ & 0.491910 & 0.047270 & -1.238700 \\
\hline$N$ & 1.936320 & -1.399240 & -1.452240 \\
\hline C & 1.740030 & -2.760960 & -1.493950 \\
\hline $\mathrm{C}$ & 3.016840 & -3.454390 & -1.509050 \\
\hline C & 3.988030 & -2.489700 & -1.452160 \\
\hline C & 3.299580 & -1.210500 & -1.423540 \\
\hline C & -2.965910 & 0.052500 & -1.428600 \\
\hline C & -2.316980 & -1.200580 & -1.465680 \\
\hline C & -3.011930 & -2.475970 & -1.489550 \\
\hline C & -2.045040 & -3.446260 & -1.522870 \\
\hline C & -0.764990 & -2.757860 & -1.517590 \\
\hline $\mathrm{N}$ & -0.954470 & -1.394880 & -1.490190 \\
\hline $\mathrm{C}$ & 0.486780 & -3.411680 & -1.520800 \\
\hline $\mathrm{C}$ & 3.951060 & 0.041890 & -1.378490 \\
\hline $\mathrm{H}$ & -4.090010 & 2.727090 & -1.494080 \\
\hline $\mathrm{H}$ & -2.168750 & 4.636710 & -1.581770 \\
\hline $\mathrm{H}$ & -4.100770 & -2.610090 & -1.493670 \\
\hline $\mathrm{H}$ & -2.187100 & -4.533570 & -1.546010 \\
\hline $\mathrm{H}$ & 5.075240 & -2.633860 & -1.442050 \\
\hline $\mathrm{H}$ & 3.154460 & -4.541080 & -1.565690 \\
\hline $\mathrm{H}$ & 3.170330 & 4.630100 & -1.49659 \\
\hline $\mathrm{H}$ & 5.084850 & 2.709740 & -1.433510 \\
\hline $\mathrm{C}$ & 0.497050 & 5.002090 & -1.514180 \\
\hline $\mathrm{C}$ & 5.441590 & 0.042570 & -1.2759 \\
\hline
\end{tabular}

trans- $\beta$

$E_{P B E / S V P}=-6003.116744246$ Hartree NImag $=0$

\begin{tabular}{|c|c|c|c|}
\hline$C$ & -1.270620 & -0.203560 & 6.243670 \\
\hline C & -0.608360 & -0.183760 & 7.492110 \\
\hline C & -1.341340 & -0.271140 & 8.682910 \\
\hline C & -2.741700 & -0.378390 & 8.637000 \\
\hline C & -3.406810 & -0.398210 & 7.394160 \\
\hline$C$ & -2.682680 & -0.312010 & 6.202010 \\
\hline $\mathrm{N}$ & -0.442600 & -0.110230 & 5.104760 \\
\hline $\mathrm{N}$ & -1.062150 & -0.136850 & 3.987970 \\
\hline$C$ & -0.302180 & -0.049120 & 2.854100 \\
\hline$C$ & -0.804950 & -0.067280 & 1.545840 \\
\hline $\mathrm{N}$ & 1.095200 & 0.073450 & 2.714470 \\
\hline $\mathrm{N}$ & 0.224110 & 0.037950 & 0.658780 \\
\hline$C$ & 1.346660 & 0.120800 & 1.383810 \\
\hline $\mathrm{H}$ & 0.490070 & -0.098670 & 7.496610 \\
\hline $\mathrm{H}$ & -0.818940 & -0.255720 & 9.653460 \\
\hline $\mathrm{H}$ & -3.320960 & -0.447100 & 9.572440 \\
\hline $\mathrm{H}$ & -4.505880 & -0.482470 & 7.362160 \\
\hline $\mathrm{H}$ & -3.178890 & -0.325600 & 5.219870 \\
\hline $\mathrm{H}$ & -1.854830 & -0.155110 & 1.240560 \\
\hline $\mathrm{H}$ & 2.360660 & 0.217310 & 0.970540 \\
\hline$C$ & 2.121720 & 0.138780 & 3.748850 \\
\hline$C$ & 1.439520 & 2.850170 & -1.633240 \\
\hline $\mathrm{N}$ & 1.626800 & 1.486490 & -1.611900 \\
\hline$C$ & 2.720510 & 3.535790 & -1.640070 \\
\hline$C$ & 3.686310 & 2.563380 & -1.617540 \\
\hline$C$ & 2.989320 & 1.289270 & -1.596740 \\
\hline$C$ & 0.188780 & 3.506140 & -1.637160 \\
\hline$C$ & -1.065210 & 2.857180 & -1.622900 \\
\hline$C$ & -2.340730 & 3.553070 & -1.647890 \\
\hline$C$ & -3.313550 & 2.589510 & -1.613660 \\
\hline$C$ & -2.627390 & 1.308890 & -1.586940 \\
\hline $\mathrm{N}$ & -1.263700 & 1.495390 & -1.593920 \\
\hline $\mathrm{Ni}$ & 0.174340 & 0.045530 & -1.375050 \\
\hline $\mathrm{N}$ & 1.616930 & -1.404260 & -1.613930 \\
\hline$C$ & 1.418640 & -2.766070 & -1.641380 \\
\hline$C$ & 2.693730 & -3.461750 & -1.671880 \\
\hline$C$ & 3.667200 & -2.498080 & -1.637160 \\
\hline$C$ & 2.980780 & -1.217850 & -1.606270 \\
\hline$C$ & -3.282520 & 0.058130 & -1.565090 \\
\hline$C$ & -2.635000 & -1.196890 & -1.588430 \\
\hline$C$ & -3.331490 & -2.471610 & -1.623530 \\
\hline$C$ & -2.366400 & -3.443730 & -1.644700 \\
\hline$C$ & -1.085600 & -2.757950 & -1.629190 \\
\hline $\mathrm{N}$ & -1.273060 & -1.394340 & -1.597470 \\
\hline$C$ & 0.164280 & -3.414490 & -1.643940 \\
\hline$C$ & 3.634960 & 0.033700 & -1.571900 \\
\hline $\mathrm{H}$ & -4.400640 & 2.734460 & -1.614960 \\
\hline $\mathrm{H}$ & -2.475860 & 4.640510 & -1.694680 \\
\hline $\mathrm{H}$ & -4.419950 & -2.605790 & -1.643460 \\
\hline $\mathrm{H}$ & -2.510360 & -4.530680 & -1.671630 \\
\hline $\mathrm{H}$ & 4.754290 & -2.643500 & -1.644730 \\
\hline $\mathrm{H}$ & 2.828510 & -4.549110 & -1.722360 \\
\hline $\mathrm{H}$ & 2.864370 & 4.622970 & -1.660250 \\
\hline $\mathrm{H}$ & 4.775280 & 2.696480 & -1.629960 \\
\hline$C$ & 0.190990 & 5.000820 & -1.651850 \\
\hline
\end{tabular}




\begin{tabular}{|c|c|c|c|c|c|c|c|}
\hline C & -4.455220 & 0.060400 & -1.296720 & C & 5.125770 & 0.023950 & -1.467950 \\
\hline C & 0.487120 & -4.906250 & -1.536830 & C & -4.775360 & 0.058390 & -1.500820 \\
\hline$c$ & 0.850920 & -5.647250 & -0.395680 & C & 0.162790 & -4.909340 & -1.655410 \\
\hline C & 0.856610 & -7.052130 & -0.395060 & C & 0.528290 & -5.646200 & -0.512240 \\
\hline C & 0.491900 & -7.745210 & -1.561730 & C & 0.535280 & -7.051140 & -0.507270 \\
\hline C & 0.125000 & -7.032970 & -2.716090 & C & 0.170530 & -7.747950 & -1.671730 \\
\hline C & 0.125720 & -5.627710 & -2.691250 & C & -0.197980 & -7.039600 & -2.828010 \\
\hline $\mathrm{F}$ & 1.201440 & -5.012560 & 0.729410 & C & -0.198540 & -5.634270 & -2.807480 \\
\hline $\mathrm{F}$ & 1.199870 & -7.732860 & 0.701780 & $\mathrm{~F}$ & 0.880000 & -5.007100 & 0.609880 \\
\hline $\mathrm{F}$ & 0.494380 & -9.078590 & -1.573170 & $\mathrm{~F}$ & 0.880360 & -7.728320 & 0.591080 \\
\hline $\mathrm{F}$ & -0.215700 & -7.695920 & -3.823990 & $\mathrm{~F}$ & 0.174510 & -9.081290 & -1.679330 \\
\hline $\mathrm{F}$ & -0.226090 & -4.974370 & -3.803380 & $\mathrm{~F}$ & -0.538950 & -7.706180 & -3.933560 \\
\hline C & -5.065180 & -0.206620 & -0.056210 & $\mathrm{~F}$ & -0.551820 & -4.984290 & -3.921140 \\
\hline C & -6.457270 & -0.152090 & 0.115540 & C & -5.443820 & -0.294050 & -0.312760 \\
\hline C & -7.275370 & 0.161160 & -0.983510 & C & -6.845600 & -0.303720 & -0.224100 \\
\hline C & -6.695800 & 0.422720 & -2.237310 & C & -7.608980 & 0.047010 & -1.350950 \\
\hline C & -5.298600 & 0.367980 & -2.380490 & C & -6.969440 & 0.404470 & -2.550270 \\
\hline $\mathrm{F}$ & -4.311130 & -0.504670 & 1.011910 & C & -5.565490 & 0.406500 & -2.612780 \\
\hline $\mathrm{F}$ & -7.004240 & -0.388040 & 1.313000 & $\mathrm{~F}$ & -4.737830 & -0.627880 & 0.776740 \\
\hline $\mathrm{F}$ & -8.599490 & 0.214540 & -0.836440 & $\mathrm{~F}$ & -7.456650 & -0.636490 & 0.916140 \\
\hline $\mathrm{F}$ & -7.473320 & 0.721470 & -3.280440 & $\mathrm{~F}$ & -8.940420 & 0.042040 & -1.281290 \\
\hline $\mathrm{F}$ & -4.774360 & 0.628120 & -3.582110 & $\mathrm{~F}$ & -7.698020 & 0.734920 & -3.618730 \\
\hline C & 6.079070 & 0.376530 & -0.066170 & $\mathrm{~F}$ & -4.983250 & 0.749960 & -3.766320 \\
\hline C & 7.477220 & 0.380260 & 0.063130 & C & 5.762440 & 0.297130 & -0.243120 \\
\hline C & 8.271050 & 0.042190 & -1.046690 & C & 7.158700 & 0.257080 & -0.103390 \\
\hline C & 7.663630 & -0.296780 & -2.267980 & C & 7.951950 & -0.058000 & -1.220490 \\
\hline c & 6.261910 & -0.292230 & -2.369970 & C & 7.345660 & -0.331000 & -2.458710 \\
\hline $\mathrm{F}$ & 5.344020 & 0.696850 & 1.012080 & C & 5.945270 & -0.284780 & -2.569820 \\
\hline $\mathrm{F}$ & 8.055610 & 0.694210 & 1.225970 & $\mathrm{~F}$ & 5.027080 & 0.595090 & 0.843350 \\
\hline $\mathrm{F}$ & 9.599850 & 0.040570 & -0.939300 & $\mathrm{~F}$ & 7.735180 & 0.506770 & 1.075440 \\
\hline $\mathrm{F}$ & 8.419270 & -0.616990 & -3.320220 & $\mathrm{~F}$ & 9.279030 & -0.101280 & -1.103490 \\
\hline $\mathrm{F}$ & 5.712220 & -0.618540 & -3.543640 & $\mathrm{~F}$ & 8.100780 & -0.631650 & -3.516840 \\
\hline C & 0.856720 & 5.726460 & -2.667050 & $\mathrm{~F}$ & 5.394880 & -0.551310 & -3.757710 \\
\hline C & 0.854060 & 7.131800 & -2.688750 & C & 0.551070 & 5.721500 & -2.807130 \\
\hline C & 0.486250 & 7.840520 & -1.532480 & C & 0.551810 & 7.126690 & -2.832800 \\
\hline C & 0.124240 & 7.144180 & -0.366910 & C & 0.186260 & 7.839430 & -1.678290 \\
\hline C & 0.133010 & 5.739190 & -0.370750 & C & -0.176700 & 7.147050 & -0.510610 \\
\hline $\mathrm{F}$ & 1.210580 & 5.075910 & -3.780180 & C & -0.170850 & 5.742030 & -0.510290 \\
\hline $\mathrm{F}$ & 1.193040 & 7.798110 & -3.795160 & $\mathrm{~F}$ & 0.901580 & 5.067240 & -3.919170 \\
\hline $\mathrm{F}$ & 0.480860 & 9.173940 & -1.540970 & $\mathrm{~F}$ & 0.891540 & 7.788980 & -3.941350 \\
\hline $\mathrm{F}$ & -0.219140 & 7.821880 & 0.731740 & $\mathrm{~F}$ & 0.184030 & 9.172740 & -1.690440 \\
\hline $\mathrm{F}$ & -0.214830 & 5.100460 & 0.752570 & $\mathrm{~F}$ & -0.518290 & 7.828460 & 0.586140 \\
\hline C & 1.863600 & 0.171530 & 4.128440 & $\mathrm{~F}$ & -0.518950 & 5.107470 & 0.615300 \\
\hline $\mathrm{H}$ & 1.565750 & 1.046340 & 4.742640 & $\mathrm{H}$ & 3.107010 & 0.230630 & 3.245750 \\
\hline $\mathrm{H}$ & 1.741700 & -0.746620 & 4.739260 & $\mathrm{H}$ & 2.096890 & -0.774400 & 4.375120 \\
\hline $\mathrm{H}$ & 2.923770 & 0.276670 & 3.822970 & $\mathrm{H}$ & 1.948670 & 1.009870 & 4.410560 \\
\hline
\end{tabular}




\section{I.6.4 1-iso-Propyl-5-phenylazoimidazole $\cdot$ NiTPPF $_{20}$ (3)}

\section{cis- $\alpha$}

$E_{P B E / S V P}=-6081.554115667$ Hartree

NImag $=0$

\begin{tabular}{|c|c|c|c|}
\hline C & 1.495320 & -2.269810 & 4.089220 \\
\hline C & 0.726750 & -3.449830 & 4.172330 \\
\hline C & 1.109710 & -4.583900 & 3.441120 \\
\hline C & 2.279040 & -4.565790 & 2.662210 \\
\hline C & 3.068730 & -3.402410 & 2.614250 \\
\hline C & 2.680080 & -2.255100 & 3.317000 \\
\hline$N$ & 1.137390 & -1.154500 & 4.895970 \\
\hline $\mathrm{N}$ & 0.875790 & -0.018870 & 4.407730 \\
\hline C & 0.709740 & 0.248910 & 3.055080 \\
\hline C & 0.414740 & -0.467780 & 1.880230 \\
\hline$N$ & 0.615480 & 1.602690 & 2.692110 \\
\hline$N$ & 0.167310 & 0.412560 & 0.870760 \\
\hline $\mathrm{C}$ & 0.313570 & 1.646400 & 1.378980 \\
\hline $\mathrm{H}$ & -0.179470 & -3.455870 & 4.798050 \\
\hline $\mathrm{H}$ & 0.483030 & -5.489410 & 3.480870 \\
\hline $\mathrm{H}$ & 2.581610 & -5.462580 & 2.097930 \\
\hline $\mathrm{H}$ & 3.990610 & -3.378150 & 2.010730 \\
\hline $\mathrm{H}$ & 3.294550 & -1.341390 & 3.279940 \\
\hline $\mathrm{H}$ & 0.346810 & -1.550560 & 1.730210 \\
\hline $\mathrm{H}$ & 0.217400 & 2.572920 & 0.802440 \\
\hline C & -2.097730 & 2.626640 & -1.128820 \\
\hline$N$ & -0.908440 & 2.003920 & -1.435410 \\
\hline$C$ & -2.003450 & 4.051800 & -1.387540 \\
\hline $\mathrm{C}$ & -0.729180 & 4.284450 & -1.839210 \\
\hline $\mathrm{C}$ & -0.050990 & 3.000130 & -1.845880 \\
\hline C & -3.237560 & 1.991110 & -0.590460 \\
\hline C & -3.354100 & 0.606870 & -0.341250 \\
\hline C & -4.552240 & -0.025020 & 0.184710 \\
\hline$C$ & -4.284160 & -1.365620 & 0.261840 \\
\hline $\mathrm{C}$ & -2.925950 & -1.546180 & -0.223440 \\
\hline$N$ & -2.380360 & -0.335330 & -0.580260 \\
\hline $\mathrm{Ni}$ & -0.413720 & 0.040850 & -1.045340 \\
\hline$N$ & 1.422410 & 0.361740 & -1.911310 \\
\hline $\mathrm{C}$ & 2.413500 & -0.572010 & -2.104850 \\
\hline $\mathrm{C}$ & 3.631730 & 0.072130 & -2.567150 \\
\hline $\mathrm{C}$ & 3.365840 & 1.414570 & -2.628130 \\
\hline $\mathrm{C}$ & 1.979770 & 1.580580 & -2.224930 \\
\hline C & -2.275930 & -2.797440 & -0.318200 \\
\hline C & -0.948560 & -2.986600 & -0.763270 \\
\hline $\mathrm{C}$ & -0.314760 & -4.285300 & -0.909890 \\
\hline $\mathrm{C}$ & 0.963500 & -4.051410 & -1.342330 \\
\hline $\mathrm{C}$ & 1.100560 & -2.611590 & -1.474920 \\
\hline$N$ & -0.073230 & -1.984420 & -1.119630 \\
\hline C & 2.276720 & -1.965020 & -1.913010 \\
\hline $\mathrm{C}$ & 1.309030 & 2.822280 & -2.183530 \\
\hline $\mathrm{H}$ & -4.948660 & -2.161400 & 0.61894 \\
\hline $\mathrm{H}$ & -5.483890 & 0.489620 & 0.449540 \\
\hline $\mathrm{H}$ & -0.789550 & -5.254970 & -0.717740 \\
\hline $\mathrm{H}$ & 1.742700 & -4.791690 & -1.560050 \\
\hline $\mathrm{H}$ & 4.045190 & 2.219010 & -2.935310 \\
\hline $\mathrm{H}$ & 4.569150 & -0.437290 & -2.82131 \\
\hline $\mathrm{H}$ & -2.810150 & 4.781770 & -1.247820 \\
\hline $\mathrm{H}$ & -0.291590 & 5.241020 & -2.15085 \\
\hline
\end{tabular}

cis- $\beta$

$E_{P B E / S V P}=-6081.544586755$ Hartree NImag $=0$

\begin{tabular}{|c|c|c|c|}
\hline $\mathrm{C}$ & -0.915390 & -2.922320 & 4.444150 \\
\hline $\mathrm{C}$ & -1.680060 & -3.143900 & 5.615190 \\
\hline $\mathrm{C}$ & -2.949490 & -3.726500 & 5.531410 \\
\hline C & -3.447320 & -4.156750 & 4.287390 \\
\hline$C$ & -2.672690 & -3.984320 & 3.126210 \\
\hline$C$ & -1.422810 & -3.351380 & 3.191350 \\
\hline $\mathrm{N}$ & 0.415100 & -2.468740 & 4.641200 \\
\hline $\mathrm{N}$ & 1.060470 & -1.730170 & 3.844490 \\
\hline$C$ & 0.521770 & -1.003140 & 2.782480 \\
\hline$C$ & 1.117210 & -0.847710 & 1.527380 \\
\hline $\mathrm{N}$ & -0.624880 & -0.191730 & 2.763270 \\
\hline $\mathrm{N}$ & 0.342010 & -0.021950 & 0.759960 \\
\hline $\mathrm{C}$ & -0.670790 & 0.383060 & 1.529320 \\
\hline $\mathrm{H}$ & -1.244950 & -2.851970 & 6.584410 \\
\hline $\mathrm{H}$ & -3.544970 & -3.875190 & 6.447160 \\
\hline $\mathrm{H}$ & -4.432960 & -4.645980 & 4.225350 \\
\hline $\mathrm{H}$ & -3.041520 & -4.346450 & 2.153220 \\
\hline $\mathrm{H}$ & -0.825860 & -3.226990 & 2.274490 \\
\hline $\mathrm{H}$ & 2.023100 & -1.338020 & 1.150650 \\
\hline $\mathrm{H}$ & -1.424960 & 1.124530 & 1.236130 \\
\hline $\mathrm{C}$ & 3.407690 & 1.610180 & -1.059510 \\
\hline $\mathrm{N}$ & 2.601290 & 0.523380 & -1.310520 \\
\hline$C$ & 4.806450 & 1.217950 & -1.081310 \\
\hline $\mathrm{C}$ & 4.833090 & -0.122860 & -1.359360 \\
\hline $\mathrm{C}$ & 3.450110 & -0.543180 & -1.501830 \\
\hline$C$ & 2.958380 & 2.929060 & -0.827230 \\
\hline C & 1.604160 & 3.329290 & -0.802480 \\
\hline$C$ & 1.163840 & 4.695800 & -0.580530 \\
\hline$C$ & -0.204990 & 4.678080 & -0.620300 \\
\hline $\mathrm{C}$ & -0.592980 & 3.304330 & -0.891160 \\
\hline $\mathrm{N}$ & 0.520920 & 2.502690 & -0.996410 \\
\hline $\mathrm{Ni}$ & 0.550670 & 0.456080 & -1.206780 \\
\hline $\mathrm{N}$ & 0.606450 & -1.486390 & -1.879220 \\
\hline$C$ & -0.474240 & -2.302200 & -2.121020 \\
\hline$C$ & -0.029710 & -3.646920 & -2.446920 \\
\hline$C$ & 1.338290 & -3.634970 & -2.376170 \\
\hline $\mathrm{C}$ & 1.721800 & -2.280860 & -2.016600 \\
\hline $\mathrm{C}$ & -1.930070 & 2.871870 & -1.033840 \\
\hline $\mathrm{C}$ & -2.330790 & 1.555360 & -1.352460 \\
\hline$C$ & -3.713090 & 1.135640 & -1.501540 \\
\hline$C$ & -3.685480 & -0.206720 & -1.777090 \\
\hline $\mathrm{C}$ & -2.286190 & -0.595700 & -1.810900 \\
\hline $\mathrm{N}$ & -1.481800 & 0.491080 & -1.554490 \\
\hline$C$ & -1.829430 & -1.907820 & -2.068300 \\
\hline $\mathrm{C}$ & 3.054830 & -1.859540 & -1.822590 \\
\hline $\mathrm{H}$ & -0.889370 & 5.525230 & -0.490710 \\
\hline $\mathrm{H}$ & 1.817820 & 5.561660 & -0.422170 \\
\hline $\mathrm{H}$ & -4.592810 & 1.785400 & -1.416890 \\
\hline $\mathrm{H}$ & -4.541140 & -0.870620 & -1.948820 \\
\hline $\mathrm{H}$ & 2.027720 & -4.468510 & -2.556900 \\
\hline $\mathrm{H}$ & -0.679670 & -4.490220 & -2.710600 \\
\hline $\mathrm{H}$ & 5.660380 & 1.883260 & -0.906140 \\
\hline $\mathrm{H}$ & 5.712140 & -0.769080 & -1.471970 \\
\hline
\end{tabular}




\begin{tabular}{|c|c|c|c|}
\hline$C$ & -4.404370 & 2.851220 & -0.225530 \\
\hline C & 2.104580 & 4.055980 & -2.462730 \\
\hline C & -3.056230 & -4.010190 & 0.066950 \\
\hline C & 3.461950 & -2.821180 & -2.223670 \\
\hline C & 4.594300 & -2.842500 & -1.388080 \\
\hline C & 5.719810 & -3.629890 & -1.680830 \\
\hline C & 5.724680 & -4.422000 & -2.841490 \\
\hline C & 4.605030 & -4.429340 & -3.690370 \\
\hline C & 3.493070 & -3.630470 & -3.375840 \\
\hline $\mathrm{F}$ & 4.617000 & -2.101530 & -0.270530 \\
\hline $\mathrm{F}$ & 6.779960 & -3.631590 & -0.868220 \\
\hline $\mathrm{F}$ & 6.788510 & -5.169280 & -3.135480 \\
\hline $\mathrm{F}$ & 4.609770 & -5.185720 & -4.789910 \\
\hline $\mathrm{F}$ & 2.443830 & -3.653050 & -4.203650 \\
\hline C & -2.725200 & -4.769100 & 1.205780 \\
\hline C & -3.452390 & -5.911900 & 1.574680 \\
\hline C & -4.553370 & -6.310650 & 0.798210 \\
\hline C & -4.911260 & -5.571790 & -0.341870 \\
\hline C & -4.160810 & -4.438810 & -0.695320 \\
\hline $\mathrm{F}$ & -1.690550 & -4.405240 & 1.976810 \\
\hline $\mathrm{F}$ & -3.111310 & -6.614160 & 2.659050 \\
\hline $\mathrm{F}$ & -5.257740 & -7.388700 & 1.141800 \\
\hline $\mathrm{F}$ & -5.955480 & -5.952060 & -1.081200 \\
\hline $\mathrm{F}$ & -4.520050 & -3.761870 & -1.790570 \\
\hline C & 2.483030 & 4.912980 & -1.411490 \\
\hline C & 3.226830 & 6.082380 & -1.634220 \\
\hline C & 3.604750 & 6.417220 & -2.946560 \\
\hline C & 3.241190 & 5.582240 & -4.016860 \\
\hline C & 2.500110 & 4.414880 & -3.764930 \\
\hline $\mathrm{F}$ & 2.133750 & 4.618240 & -0.146060 \\
\hline $\mathrm{F}$ & 3.575710 & 6.872330 & -0.615430 \\
\hline $\mathrm{F}$ & 4.307610 & 7.526110 & -3.176770 \\
\hline $\mathrm{F}$ & 3.596590 & 5.905080 & -5.261900 \\
\hline $\mathrm{F}$ & 2.168820 & 3.640600 & -4.802140 \\
\hline C & -5.282710 & 3.358330 & -1.201590 \\
\hline C & -6.396110 & 4.143370 & -0.855910 \\
\hline C & -6.641430 & 4.437640 & 0.496070 \\
\hline C & -5.776210 & 3.951330 & 1.491440 \\
\hline C & -4.669490 & 3.170430 & 1.119880 \\
\hline $\mathrm{F}$ & -5.069870 & 3.092320 & -2.494050 \\
\hline $\mathrm{F}$ & -7.221660 & 4.607960 & -1.796360 \\
\hline $\mathrm{F}$ & -7.696400 & 5.178320 & 0.834930 \\
\hline $\mathrm{F}$ & -6.010750 & 4.237600 & 2.774640 \\
\hline $\mathrm{F}$ & -3.855640 & 2.722050 & 2.087160 \\
\hline C & 0.842530 & 2.758480 & 3.587040 \\
\hline C & 2.297190 & 3.237420 & 3.485520 \\
\hline $\mathrm{H}$ & 2.474170 & 4.060870 & 4.210760 \\
\hline $\mathrm{H}$ & 3.006700 & 2.415490 & 3.721270 \\
\hline $\mathrm{H}$ & 2.521150 & 3.619980 & 2.464570 \\
\hline C & -0.170200 & 3.873160 & 3.300800 \\
\hline $\mathrm{H}$ & -0.099670 & 4.644890 & 4.096880 \\
\hline $\mathrm{H}$ & 0.034660 & 4.378330 & 2.330770 \\
\hline $\mathrm{H}$ & -1.211940 & 3.486050 & 3.282330 \\
\hline $\mathrm{H}$ & 0.669470 & 2.343020 & 4.601820 \\
\hline
\end{tabular}

\begin{tabular}{|c|c|c|c|}
\hline C & 3.996420 & 3.985000 & -0.624560 \\
\hline C & 4.130890 & -2.890340 & -1.944070 \\
\hline$C$ & -3.006350 & 3.886950 & -0.824980 \\
\hline C & -2.860270 & -2.969970 & -2.265280 \\
\hline C & -3.060720 & -3.965980 & -1.291270 \\
\hline C & -4.038530 & -4.962290 & -1.430980 \\
\hline C & -4.841590 & -4.981770 & -2.584040 \\
\hline C & -4.661720 & -4.005180 & -3.578680 \\
\hline C & -3.677420 & -3.016390 & -3.410880 \\
\hline$F$ & -2.312640 & -3.971670 & -0.176280 \\
\hline$F$ & -4.214100 & -5.879510 & -0.474080 \\
\hline$F$ & -5.773420 & -5.922430 & -2.734350 \\
\hline$F$ & -5.422810 & -4.025590 & -4.674510 \\
\hline$F$ & -3.530570 & -2.101440 & -4.373560 \\
\hline C & -3.791930 & 3.890260 & 0.342090 \\
\hline C & -4.802010 & 4.841700 & 0.556620 \\
\hline$C$ & -5.036140 & 5.827400 & -0.418450 \\
\hline C & -4.264980 & 5.852610 & -1.592990 \\
\hline C & -3.265210 & 4.884260 & -1.784440 \\
\hline$F$ & -3.577150 & 2.967240 & 1.298680 \\
\hline$F$ & -5.532990 & 4.824550 & 1.674050 \\
\hline $\mathrm{F}$ & -5.987700 & 6.740350 & -0.226980 \\
\hline$F$ & -4.487310 & 6.790170 & -2.515650 \\
\hline$F$ & -2.546920 & 4.928910 & -2.909540 \\
\hline C & 4.731840 & -3.435870 & -0.793980 \\
\hline C & 5.740070 & -4.410650 & -0.876060 \\
\hline C & 6.166340 & -4.854380 & -2.140050 \\
\hline C & 5.585580 & -4.325460 & -3.305290 \\
\hline C & 4.576590 & -3.353620 & -3.196070 \\
\hline$F$ & 4.339480 & -3.029750 & 0.421260 \\
\hline$F$ & 6.289130 & -4.918410 & 0.229760 \\
\hline$F$ & 7.122250 & -5.778610 & -2.236880 \\
\hline$F$ & 5.997390 & -4.748640 & -4.502610 \\
\hline$F$ & 4.039280 & -2.868440 & -4.319750 \\
\hline C & 4.812770 & 4.409710 & -1.691710 \\
\hline C & 5.795760 & 5.399000 & -1.525780 \\
\hline C & 5.974130 & 5.990830 & -0.263950 \\
\hline C & 5.172170 & 5.590590 & 0.818290 \\
\hline C & 4.197520 & 4.597040 & 0.627560 \\
\hline$F$ & 4.662210 & 3.869210 & -2.905010 \\
\hline$F$ & 6.554180 & 5.781600 & -2.555800 \\
\hline$F$ & 6.903460 & 6.930820 & -0.093000 \\
\hline$F$ & 5.344030 & 6.152070 & 2.017740 \\
\hline$F$ & 3.451600 & 4.236850 & 1.678260 \\
\hline C & -1.463870 & 0.235260 & 3.910600 \\
\hline C & -2.949010 & -0.020910 & 3.630000 \\
\hline $\mathrm{H}$ & -3.543810 & 0.227540 & 4.534960 \\
\hline $\mathrm{H}$ & -3.319020 & 0.617100 & 2.797240 \\
\hline $\mathrm{H}$ & -3.132100 & -1.085910 & 3.375740 \\
\hline C & -1.173100 & 1.700150 & 4.270040 \\
\hline $\mathrm{H}$ & -1.522150 & 2.388540 & 3.469210 \\
\hline $\mathrm{H}$ & -1.709880 & 1.968820 & 5.205490 \\
\hline $\mathrm{H}$ & -0.086870 & 1.867440 & 4.433830 \\
\hline $\mathrm{H}$ & -1.146060 & -0.403900 & 4.757670 \\
\hline
\end{tabular}




\section{trans- $\alpha$}

$E_{P B E / S V P}=-6081.569156201$ Hartree NImag $=0$

\begin{tabular}{|c|c|c|c|}
\hline C & -2.856990 & 1.587200 & 4.953370 \\
\hline C & -4.271200 & 1.584940 & 4.946050 \\
\hline C & -4.985740 & 1.936010 & 6.098530 \\
\hline C & -4.294620 & 2.290140 & 7.269780 \\
\hline C & -2.884560 & 2.292830 & 7.283770 \\
\hline C & -2.164880 & 1.945310 & 6.137540 \\
\hline$N$ & -2.247530 & 1.211420 & 3.741120 \\
\hline $\mathrm{N}$ & -0.972260 & 1.240930 & 3.734650 \\
\hline C & -0.392760 & 0.853330 & 2.551360 \\
\hline C & -0.874740 & 0.402790 & 1.313430 \\
\hline $\mathrm{N}$ & 1.002190 & 0.852820 & 2.436400 \\
\hline$N$ & 0.182850 & 0.142750 & 0.493410 \\
\hline C & 1.296570 & 0.418910 & 1.190130 \\
\hline $\mathrm{H}$ & -4.778290 & 1.300560 & 4.010550 \\
\hline $\mathrm{H}$ & -6.087810 & 1.932780 & 6.085850 \\
\hline $\mathrm{H}$ & -4.853830 & 2.564440 & 8.179450 \\
\hline $\mathrm{H}$ & -2.347040 & 2.569240 & 8.206280 \\
\hline $\mathrm{H}$ & -1.064490 & 1.938810 & 6.130280 \\
\hline $\mathrm{H}$ & -1.917100 & 0.272630 & 1.003470 \\
\hline $\mathrm{H}$ & 2.315950 & 0.307180 & 0.803390 \\
\hline C & 3.025390 & 0.573430 & -1.981950 \\
\hline$N$ & 2.229250 & -0.500150 & -1.647930 \\
\hline C & 4.427830 & 0.218240 & -1.861230 \\
\hline C & 4.466590 & -1.085450 & -1.437250 \\
\hline C & 3.087630 & -1.517180 & -1.294420 \\
\hline C & 2.559010 & 1.855630 & -2.346540 \\
\hline C & 1.201560 & 2.219980 & -2.475330 \\
\hline C & 0.747390 & 3.542860 & -2.869840 \\
\hline C & -0.621880 & 3.501070 & -2.871170 \\
\hline C & -0.995200 & 2.149480 & -2.488910 \\
\hline$N$ & 0.127240 & 1.388810 & -2.259160 \\
\hline $\mathrm{Ni}$ & 0.179260 & -0.498140 & -1.439580 \\
\hline$N$ & 0.247680 & -2.515510 & -1.043310 \\
\hline C & -0.826330 & -3.351440 & -0.843250 \\
\hline C & -0.372820 & -4.661170 & -0.405890 \\
\hline C & 0.992910 & -4.596000 & -0.325920 \\
\hline C & 1.368290 & -3.253730 & -0.737670 \\
\hline C & -2.327650 & 1.696010 & -2.378490 \\
\hline C & -2.710320 & 0.381090 & -2.036950 \\
\hline C & -4.085760 & -0.083670 & -2.009320 \\
\hline C & -4.045440 & -1.404460 & -1.645670 \\
\hline C & -2.645020 & -1.739570 & -1.452460 \\
\hline $\mathrm{N}$ & -1.851850 & -0.641720 & -1.701030 \\
\hline$C$ & -2.181870 & -3.009010 & -1.042020 \\
\hline C & 2.700540 & -2.795890 & -0.834460 \\
\hline $\mathrm{H}$ & -1.318210 & 4.312140 & -3.116230 \\
\hline $\mathrm{H}$ & 1.393830 & 4.392150 & -3.123100 \\
\hline $\mathrm{H}$ & -4.968420 & 0.521430 & -2.250020 \\
\hline $\mathrm{H}$ & -4.890850 & -2.091420 & -1.518130 \\
\hline $\mathrm{H}$ & 1.684970 & -5.392340 & -0.026540 \\
\hline $\mathrm{H}$ & -1.016650 & -5.523320 & -0.193830 \\
\hline $\mathrm{H}$ & 5.276680 & 0.877490 & -2.079210 \\
\hline $\mathrm{H}$ & 5.353350 & -1.704150 & -1.253120 \\
\hline
\end{tabular}

\section{trans- $\beta$}

$E_{P B E / S V P}=-6081.569660356$ Hartree NImag $=0$

\begin{tabular}{|c|c|c|c|}
\hline C & 1.271090 & -5.652030 & 2.156170 \\
\hline C & 1.913160 & -6.287890 & 3.242750 \\
\hline C & 1.972460 & -7.686200 & 3.308940 \\
\hline C & 1.391850 & -8.461130 & 2.290740 \\
\hline C & 0.750500 & -7.831090 & 1.204940 \\
\hline C & 0.686830 & -6.436940 & 1.131670 \\
\hline$N$ & 1.265550 & -4.240660 & 2.181160 \\
\hline$N$ & 0.674910 & -3.688240 & 1.194160 \\
\hline C & 0.623560 & -2.320450 & 1.136050 \\
\hline C & -0.011350 & -1.621560 & 0.098100 \\
\hline$N$ & 1.130850 & -1.325650 & 1.997550 \\
\hline$N$ & 0.100000 & -0.281790 & 0.310910 \\
\hline C & 0.787160 & -0.133940 & 1.451840 \\
\hline $\mathrm{H}$ & 2.360010 & -5.656200 & 4.027190 \\
\hline $\mathrm{H}$ & 2.474570 & -8.175940 & 4.159320 \\
\hline $\mathrm{H}$ & 1.437840 & -9.561470 & 2.340010 \\
\hline $\mathrm{H}$ & 0.296160 & -8.442410 & 0.407390 \\
\hline $\mathrm{H}$ & 0.192360 & -5.922260 & 0.294100 \\
\hline $\mathrm{H}$ & -0.522880 & -2.051560 & -0.771800 \\
\hline $\mathrm{H}$ & 1.053490 & 0.834070 & 1.895900 \\
\hline C & -2.530480 & 2.340350 & 1.216820 \\
\hline$N$ & -1.273130 & 2.426590 & 0.664260 \\
\hline C & -2.617600 & 3.169500 & 2.406990 \\
\hline C & -1.389420 & 3.756050 & 2.567530 \\
\hline C & -0.559010 & 3.279830 & 1.475400 \\
\hline C & -3.593090 & 1.554100 & 0.717070 \\
\hline C & -3.527790 & 0.745280 & -0.438580 \\
\hline C & -4.650260 & -0.013280 & -0.964080 \\
\hline C & -4.196080 & -0.660060 & -2.082720 \\
\hline C & -2.800980 & -0.285640 & -2.240500 \\
\hline$N$ & -2.417890 & 0.570530 & -1.233420 \\
\hline $\mathrm{Ni}$ & -0.520810 & 1.260040 & -0.859210 \\
\hline$N$ & 1.253140 & 2.298900 & -0.737410 \\
\hline C & 2.365350 & 2.120380 & -1.527350 \\
\hline C & 3.461910 & 2.945610 & -1.049700 \\
\hline C & 3.000270 & 3.609180 & 0.057350 \\
\hline C & 1.622030 & 3.191290 & 0.243660 \\
\hline C & -1.966640 & -0.752470 & -3.279940 \\
\hline C & -0.611360 & -0.395310 & -3.455880 \\
\hline C & 0.225640 & -0.881840 & -4.539360 \\
\hline C & 1.465940 & -0.331860 & -4.352730 \\
\hline C & 1.375940 & 0.504540 & -3.168000 \\
\hline $\mathrm{N}$ & 0.105180 & 0.450510 & -2.640660 \\
\hline C & 2.446650 & 1.267570 & -2.651140 \\
\hline C & 0.794670 & 3.637330 & 1.296680 \\
\hline $\mathrm{H}$ & -4.758050 & -1.330490 & -2.744020 \\
\hline $\mathrm{H}$ & -5.660600 & -0.038460 & -0.537780 \\
\hline $\mathrm{H}$ & -0.095500 & -1.552860 & -5.345250 \\
\hline $\mathrm{H}$ & 2.361200 & -0.476790 & -4.969110 \\
\hline $\mathrm{H}$ & 3.537970 & 4.334250 & 0.680860 \\
\hline $\mathrm{H}$ & 4.453230 & 3.021880 & -1.512960 \\
\hline $\mathrm{H}$ & -3.504100 & 3.288160 & 3.041500 \\
\hline $\mathrm{H}$ & -1.074840 & 4.454560 & 3.352840 \\
\hline
\end{tabular}




\begin{tabular}{|c|c|c|c|c|c|c|c|}
\hline C & 3.583560 & 2.921880 & -2.562770 & C & -4.875940 & 1.565020 & 1.483630 \\
\hline C & 3.794260 & -3.714960 & -0.396440 & C & 1.424080 & 4.497610 & 2.346670 \\
\hline C & -3.416100 & 2.690110 & -2.628110 & C & -2.557360 & -1.709260 & -4.263690 \\
\hline C & -3.206780 & -4.069200 & -0.794010 & C & 3.772200 & 1.155770 & -3.331590 \\
\hline 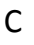 & -3.543800 & -4.465920 & 0.514080 & C & 4.849430 & 0.495750 & -2.708400 \\
\hline C & -4.510350 & -5.455020 & 0.762180 & C & 6.103850 & 0.375000 & -3.327360 \\
\hline C & -5.158720 & -6.073580 & -0.320370 & C & 6.299420 & 0.922930 & -4.606240 \\
\hline C & -4.840370 & -5.699910 & -1.636890 & C & 5.244310 & 1.589350 & -5.252130 \\
\hline C & -3.873630 & -4.704880 & -1.859000 & C & 3.998950 & 1.698600 & -4.610830 \\
\hline $\mathrm{F}$ & -2.942190 & -3.891540 & 1.561830 & $\mathrm{~F}$ & 4.692800 & -0.042040 & -1.492700 \\
\hline $\mathrm{F}$ & -4.818590 & -5.806820 & 2.013370 & $\mathrm{~F}$ & 7.106170 & -0.259410 & -2.713820 \\
\hline $\mathrm{F}$ & -6.077240 & -7.014630 & -0.100750 & $\mathrm{~F}$ & 7.485600 & 0.809860 & -5.204440 \\
\hline $\mathrm{F}$ & -5.454900 & -6.294440 & -2.662340 & $\mathrm{~F}$ & 5.431540 & 2.116470 & -6.464450 \\
\hline $\mathrm{F}$ & -3.591100 & -4.368410 & -3.121480 & $\mathrm{~F}$ & 3.015300 & 2.340030 & -5.249670 \\
\hline C & -4.128050 & 3.259780 & -1.554910 & C & -2.200280 & -3.071150 & -4.258100 \\
\hline C & -5.132880 & 4.219480 & -1.761100 & C & -2.736500 & -3.981570 & -5.182970 \\
\hline C & -5.450710 & 4.615030 & -3.071620 & C & -3.658790 & -3.528470 & -6.141710 \\
\hline C & -4.763640 & 4.055290 & -4.162530 & C & -4.037920 & -2.175510 & -6.168570 \\
\hline C & -3.755640 & 3.103880 & -3.929890 & C & -3.485680 & -1.284590 & -5.233280 \\
\hline $\mathrm{F}$ & -3.842360 & 2.901120 & -0.297810 & $\mathrm{~F}$ & -1.327140 & -3.531460 & -3.351440 \\
\hline $\mathrm{F}$ & -5.782970 & 4.760630 & -0.727280 & $\mathrm{~F}$ & -2.382620 & -5.269070 & -5.157060 \\
\hline $\mathrm{F}$ & -6.403380 & 5.524540 & -3.278140 & $\mathrm{~F}$ & -4.173600 & -4.384320 & -7.024650 \\
\hline $\mathrm{F}$ & -5.073750 & 4.427960 & -5.406820 & $\mathrm{~F}$ & -4.912780 & -1.745380 & -7.080710 \\
\hline $\mathrm{F}$ & -3.111670 & 2.591650 & -4.983650 & $\mathrm{~F}$ & -3.861860 & -0.002880 & -5.282150 \\
\hline C & 4.505550 & -3.464460 & 0.792770 & C & 2.112530 & 3.909220 & 3.422330 \\
\hline C & 5.553610 & -4.293120 & 1.222910 & C & 2.716370 & 4.672920 & 4.432450 \\
\hline C & 5.907930 & -5.411560 & 0.449290 & C & 2.636380 & 6.075260 & 4.370090 \\
\hline C & 5.214990 & -5.689750 & -0.741000 & C & 1.954130 & 6.694050 & 3.308380 \\
\hline C & 4.171660 & -4.841960 & -1.150500 & C & 1.356500 & 5.902350 & 2.311280 \\
\hline $\mathrm{F}$ & 4.188450 & -2.403020 & 1.550870 & $\mathrm{~F}$ & 2.200580 & 2.567270 & 3.507360 \\
\hline $\mathrm{F}$ & 6.211390 & -4.029390 & 2.355520 & $\mathrm{~F}$ & 3.359390 & 4.082810 & 5.442920 \\
\hline $\mathrm{F}$ & 6.902550 & -6.206820 & 0.844000 & $\mathrm{~F}$ & 3.206380 & 6.816810 & 5.319770 \\
\hline $\mathrm{F}$ & 5.554850 & -6.751750 & -1.474500 & $\mathrm{~F}$ & 1.879530 & 8.025240 & 3.253280 \\
\hline $\mathrm{F}$ & 3.532480 & -5.129970 & -2.288580 & $\mathrm{~F}$ & 0.714420 & 6.516550 & 1.314790 \\
\hline C & 4.381820 & 2.960280 & -3.720980 & C & -5.716710 & 2.694340 & 1.502300 \\
\hline C & 5.356450 & 3.954020 & -3.913930 & C & -6.906970 & 2.719000 & 2.248620 \\
\hline C & 5.543300 & 4.939870 & -2.930250 & C & -7.276920 & 1.589570 & 2.998430 \\
\hline C & 4.759850 & 4.926490 & -1.763490 & C & -6.461390 & 0.445380 & 2.992340 \\
\hline C & 3.795460 & 3.920450 & -1.593320 & C & -5.277190 & 0.444830 & 2.237400 \\
\hline $\mathrm{F}$ & 4.226150 & 2.032200 & -4.669760 & $\mathrm{~F}$ & -5.388190 & 3.784110 & 0.801270 \\
\hline $\mathrm{F}$ & 6.103650 & 3.968390 & -5.019770 & $\mathrm{~F}$ & -7.685270 & 3.803680 & 2.250650 \\
\hline $\mathrm{F}$ & 6.465600 & 5.886680 & -3.102430 & $\mathrm{~F}$ & -8.399940 & 1.602790 & 3.716720 \\
\hline $\mathrm{F}$ & 4.942880 & 5.861080 & -0.827030 & $\mathrm{~F}$ & -6.811510 & -0.625890 & 3.708870 \\
\hline $\mathrm{F}$ & 3.073010 & 3.919630 & -0.463450 & $\mathrm{~F}$ & -4.517380 & -0.655710 & 2.256230 \\
\hline C & 1.971320 & 1.279960 & 3.468230 & C & 1.925060 & -1.478320 & 3.247330 \\
\hline C & 2.877340 & 0.111340 & 3.875320 & C & 1.179740 & -0.859920 & 4.436300 \\
\hline $\mathrm{H}$ & 3.565310 & 0.434890 & 4.685710 & $\mathrm{H}$ & 1.096370 & 0.245220 & 4.343060 \\
\hline $\mathrm{H}$ & 2.276950 & -0.744110 & 4.252640 & $\mathrm{H}$ & 0.158890 & -1.287450 & 4.536060 \\
\hline $\mathrm{H}$ & 3.496660 & -0.250890 & 3.025830 & $\mathrm{H}$ & 1.736350 & -1.076470 & 5.373560 \\
\hline C & 2.743020 & 2.518800 & 2.994480 & C & 3.334080 & -0.902250 & 3.056460 \\
\hline $\mathrm{H}$ & 3.359320 & 2.303790 & 2.093670 & $\mathrm{H}$ & 3.950550 & -1.125840 & 3.953780 \\
\hline $\mathrm{H}$ & 2.048310 & 3.348230 & 2.742130 & $\mathrm{H}$ & 3.835360 & -1.353080 & 2.173160 \\
\hline $\mathrm{H}$ & 3.423910 & 2.868410 & 3.800020 & $\mathrm{H}$ & 3.313840 & 0.202330 & 2.926770 \\
\hline $\mathrm{H}$ & 1.332400 & 1.559770 & 4.331150 & $\mathrm{H}$ & 1.992190 & -2.574110 & 3.385490 \\
\hline
\end{tabular}




\section{I.6.4 1-Trimethylsilyl-5-phenylazoimidazole · NiTPPF 20 (4)}

\begin{tabular}{|c|c|c|c|c|c|c|c|}
\hline \multicolumn{4}{|c|}{ cis- $\alpha$} & \multicolumn{4}{|c|}{ cis- $\beta$} \\
\hline \multicolumn{4}{|c|}{$E_{P B E / S V P}=-6372.055197751$ Hartree } & \multicolumn{4}{|c|}{$E_{P B E / S V P}=-6372.040582192$ Hartree } \\
\hline \multicolumn{4}{|c|}{ NImag $=0$} & \multicolumn{4}{|c|}{ NImag $=0$} \\
\hline $\mathrm{C}$ & 1.817430 & -2.612400 & 3.619650 & $\mathrm{C}$ & 1.868980 & -0.227900 & 4.824040 \\
\hline C & 1.592220 & -3.978390 & 3.346980 & $\mathrm{C}$ & 1.880260 & -0.739650 & 6.145270 \\
\hline $\mathrm{C}$ & 2.516150 & -4.699240 & 2.577480 & $\mathrm{C}$ & 2.402990 & -2.009910 & 6.411550 \\
\hline $\mathrm{C}$ & 3.690010 & -4.081180 & 2.114740 & $\mathrm{C}$ & 2.987520 & -2.761370 & 5.375150 \\
\hline $\mathrm{C}$ & 3.932720 & -2.729270 & 2.417470 & $\mathrm{C}$ & 3.032500 & -2.236590 & 4.071000 \\
\hline $\mathrm{C}$ & 3.000510 & -1.990220 & 3.157740 & $\mathrm{C}$ & 2.458990 & -0.990390 & 3.783230 \\
\hline $\mathrm{N}$ & 0.925590 & -1.934480 & 4.495590 & $\mathrm{~N}$ & 1.470430 & 1.129820 & 4.694130 \\
\hline $\mathrm{N}$ & 0.259440 & -0.916580 & 4.158080 & $\mathrm{~N}$ & 0.958670 & 1.650140 & 3.663370 \\
\hline$C$ & 0.158900 & -0.395380 & 2.871170 & $\mathrm{C}$ & 0.440930 & 0.956430 & 2.569560 \\
\hline$C$ & 0.468660 & -0.765190 & 1.549660 & $\mathrm{C}$ & 0.652860 & 1.317250 & 1.238020 \\
\hline$N$ & -0.570020 & 0.800890 & 2.779170 & $\mathrm{~N}$ & -0.427830 & -0.152900 & 2.557290 \\
\hline $\mathrm{N}$ & -0.048200 & 0.168760 & 0.699210 & $\mathrm{~N}$ & 0.003570 & 0.424860 & 0.426090 \\
\hline$C$ & -0.649670 & 1.095270 & 1.463290 & $\mathrm{C}$ & -0.647990 & -0.414020 & 1.230990 \\
\hline $\mathrm{H}$ & 0.678830 & -4.457010 & 3.733480 & $\mathrm{H}$ & 1.480920 & -0.105800 & 6.953360 \\
\hline $\mathrm{H}$ & 2.310660 & -5.755190 & 2.337610 & $\mathrm{H}$ & 2.385140 & -2.405840 & 7.440310 \\
\hline $\mathrm{H}$ & 4.422300 & -4.655440 & 1.524450 & $\mathrm{H}$ & 3.429380 & -3.748410 & 5.588070 \\
\hline $\mathrm{H}$ & 4.852640 & -2.236800 & 2.064120 & $\mathrm{H}$ & 3.527330 & -2.798140 & 3.263080 \\
\hline $\mathrm{H}$ & 3.188910 & -0.930630 & 3.394130 & $\mathrm{H}$ & 2.508570 & -0.593550 & 2.757780 \\
\hline $\mathrm{H}$ & 1.009630 & -1.642730 & 1.180390 & $\mathrm{H}$ & 1.267490 & 2.140280 & 0.854080 \\
\hline $\mathrm{H}$ & -1.142890 & 1.989120 & 1.065650 & $\mathrm{H}$ & -1.330380 & -1.197970 & 0.876890 \\
\hline$C$ & 1.489540 & 2.772180 & -1.664970 & $\mathrm{C}$ & 2.655730 & 1.817770 & -1.932870 \\
\hline $\mathrm{N}$ & 1.480670 & 1.396420 & -1.697390 & $\mathrm{~N}$ & 1.980490 & 0.621000 & -1.866120 \\
\hline$C$ & 2.851350 & 3.272140 & -1.752520 & $\mathrm{C}$ & 4.091160 & 1.591580 & -1.901380 \\
\hline $\mathrm{C}$ & 3.665010 & 2.173850 & -1.846770 & $\mathrm{C}$ & 4.271240 & 0.237020 & -1.800590 \\
\hline$C$ & 2.796050 & 1.009930 & -1.803840 & $\mathrm{C}$ & 2.945040 & -0.355790 & -1.771950 \\
\hline$C$ & 0.346480 & 3.595090 & -1.565250 & $\mathrm{C}$ & 2.051730 & 3.093900 & -1.979950 \\
\hline$C$ & -0.985210 & 3.128000 & -1.500880 & $\mathrm{C}$ & 0.658830 & 3.331800 & -1.976200 \\
\hline$C$ & -2.149940 & 3.990880 & -1.412220 & $\mathrm{C}$ & 0.059470 & 4.654960 & -2.026140 \\
\hline$C$ & -3.238930 & 3.166880 & -1.281040 & $\mathrm{C}$ & -1.295930 & 4.473340 & -1.964120 \\
\hline$C$ & -2.733090 & 1.806380 & -1.305120 & $\mathrm{C}$ & -1.520730 & 3.039070 & -1.899500 \\
\hline $\mathrm{N}$ & -1.365450 & 1.805220 & -1.462810 & $\mathrm{~N}$ & -0.319650 & 2.366270 & -1.909910 \\
\hline $\mathrm{Ni}$ & -0.125700 & 0.163390 & -1.339970 & $\mathrm{Ni}$ & -0.039620 & 0.356200 & -1.603230 \\
\hline $\mathrm{N}$ & 1.063980 & -1.468230 & -1.705970 & $\mathrm{~N}$ & 0.228560 & -1.681550 & -1.744850 \\
\hline$C$ & 0.675430 & -2.788790 & -1.743190 & $\mathrm{C}$ & -0.747070 & -2.651660 & -1.719860 \\
\hline$C$ & 1.828410 & -3.648370 & -1.948900 & $\mathrm{C}$ & -0.146990 & -3.973290 & -1.657100 \\
\hline$C$ & 2.922940 & -2.826790 & -2.018590 & $\mathrm{C}$ & 1.210490 & -3.783960 & -1.621810 \\
\hline$C$ & 2.431910 & -1.468510 & -1.865430 & $\mathrm{C}$ & 1.429480 & -2.349000 & -1.675810 \\
\hline$C$ & -3.539140 & 0.658540 & -1.148830 & $\mathrm{C}$ & -2.797080 & 2.436500 & -1.849020 \\
\hline$C$ & -3.083290 & -0.673910 & -1.225840 & $\mathrm{C}$ & -3.034910 & 1.043220 & -1.835920 \\
\hline$C$ & -3.945680 & -1.837300 & -1.109890 & $\mathrm{C}$ & -4.358120 & 0.443290 & -1.871520 \\
\hline$C$ & -3.138420 & -2.935500 & -1.249620 & $\mathrm{C}$ & -4.174800 & -0.914290 & -1.840370 \\
\hline$C$ & -1.787070 & -2.435530 & -1.443780 & $\mathrm{C}$ & -2.740320 & -1.136200 & -1.796640 \\
\hline $\mathrm{N}$ & -1.779260 & -1.060570 & -1.433630 & $\mathrm{~N}$ & -2.067490 & 0.065500 & -1.804540 \\
\hline$C$ & -0.648880 & -3.258860 & -1.597680 & $\mathrm{C}$ & -2.138260 & -2.411220 & -1.736100 \\
\hline$C$ & 3.254930 & -0.322300 & -1.883730 & $\mathrm{C}$ & 2.702410 & -1.740780 & -1.660150 \\
\hline $\mathrm{H}$ & -4.293850 & 3.454570 & -1.193430 & $\mathrm{H}$ & -2.073750 & 5.246150 & -1.968180 \\
\hline $\mathrm{H}$ & -2.138520 & 5.086900 & -1.455660 & $\mathrm{H}$ & 0.604810 & 5.603080 & -2.107420 \\
\hline $\mathrm{H}$ & -5.031190 & -1.815030 & -0.952320 & $\mathrm{H}$ & -5.307630 & 0.989190 & -1.931630 \\
\hline $\mathrm{H}$ & -3.432070 & -3.991640 & -1.217660 & $\mathrm{H}$ & -4.943540 & -1.696610 & -1.859210 \\
\hline $\mathrm{H}$ & 3.970780 & -3.113160 & -2.170790 & $\mathrm{H}$ & 1.995260 & -4.549380 & -1.578860 \\
\hline $\mathrm{H}$ & 1.801780 & -4.741000 & -2.041030 & $\mathrm{H}$ & -0.693860 & -4.924560 & -1.651920 \\
\hline $\mathrm{H}$ & 3.146900 & 4.328340 & -1.751520 & $\mathrm{H}$ & 4.863510 & 2.368820 & -1.947070 \\
\hline $\mathrm{H}$ & 4.757500 & 2.152340 & -1.943790 & $\mathrm{H}$ & 5.218990 & -0.314300 & -1.758460 \\
\hline
\end{tabular}




\begin{tabular}{|c|c|c|c|c|c|c|c|}
\hline$c$ & 0.553570 & 5.070930 & -1.454060 & C & 2.947530 & 4.289100 & -1.969970 \\
\hline E & 4.731670 & -0.534410 & -1.998840 & C & 3.884660 & -2.626640 & -1.425880 \\
\hline c & -4.976910 & 0.879940 & -0.799270 & C & -3.993220 & 3.329850 & -1.804510 \\
\hline C & -0.857770 & -4.738040 & -1.565330 & C & -3.040570 & -3.599560 & -1.636270 \\
\hline C & -0.442930 & -5.499910 & -0.456320 & C & -3.272150 & -4.224200 & -0.397510 \\
\hline C & -0.639380 & -6.888480 & -0.391080 & C & -4.104860 & -5.347240 & -0.271650 \\
\hline C & -1.273690 & -7.545530 & -1.459050 & C & -4.726480 & -5.872470 & -1.418370 \\
\hline C & -1.701680 & -6.811700 & -2.578930 & C & -4.517910 & -5.268430 & -2.670050 \\
\hline C & -1.488620 & -5.423030 & -2.621480 & C & -3.682000 & -4.142130 & -2.765560 \\
\hline $\mathrm{F}$ & 0.153450 & -4.897730 & 0.581130 & $\mathrm{~F}$ & -2.693610 & -3.738070 & 0.717390 \\
\hline $\mathrm{F}$ & -0.236870 & -7.583310 & 0.677720 & $\mathrm{~F}$ & -4.307080 & -5.917010 & 0.919200 \\
\hline $\mathrm{F}$ & -1.469950 & -8.863240 & -1.410380 & $\mathrm{~F}$ & -5.516700 & -6.941320 & -1.319090 \\
\hline $\mathrm{F}$ & -2.303950 & -7.438180 & -3.592120 & $\mathrm{~F}$ & -5.108160 & -5.770990 & -3.756370 \\
\hline $\mathrm{F}$ & -1.902420 & -4.751830 & -3.700780 & $\mathrm{~F}$ & -3.503480 & -3.586260 & -3.966630 \\
\hline C & -5.367990 & 0.949330 & 0.550520 & C & -4.801990 & 3.397030 & -0.652620 \\
\hline C & -6.701550 & 1.167270 & 0.930060 & C & -5.947860 & 4.206810 & -0.595160 \\
\hline C & -7.679790 & 1.327510 & -0.066890 & C & -6.300910 & 4.984170 & -1.711330 \\
\hline C & -7.318440 & 1.266840 & -1.423800 & C & -5.510690 & 4.943000 & -2.872270 \\
\hline C & -5.975520 & 1.042640 & -1.775760 & C & -4.372750 & 4.119990 & -2.907200 \\
\hline $\mathrm{F}$ & -4.444970 & 0.816180 & 1.519440 & $\mathrm{~F}$ & -4.488750 & 2.672470 & 0.429520 \\
\hline $\mathrm{F}$ & -7.044330 & 1.226960 & 2.219600 & $\mathrm{~F}$ & -6.698110 & 4.245610 & 0.509070 \\
\hline $\mathrm{F}$ & -8.950430 & 1.541530 & 0.274360 & $\mathrm{~F}$ & -7.385680 & 5.757840 & -1.671160 \\
\hline $\mathrm{F}$ & -8.250620 & 1.424150 & -2.365590 & $\mathrm{~F}$ & -5.849730 & 5.678260 & -3.933650 \\
\hline $\mathrm{F}$ & -5.657900 & 0.994240 & -3.072300 & $\mathrm{~F}$ & -3.648280 & 4.096020 & -4.030590 \\
\hline C & 5.554930 & -0.532470 & -0.858570 & C & 4.379770 & -2.789670 & -0.119780 \\
\hline C & 6.941640 & -0.736350 & -0.943050 & C & 5.477710 & -3.614390 & 0.166850 \\
\hline C & 7.533730 & -0.932920 & -2.202350 & C & 6.115360 & -4.295240 & -0.884300 \\
\hline C & 6.736670 & -0.935350 & -3.360080 & C & 5.646240 & -4.149470 & -2.201760 \\
\hline C & 5.349540 & -0.738200 & -3.246580 & C & 4.536790 & -3.323280 & -2.458750 \\
\hline $\mathrm{F}$ & 5.017370 & -0.342450 & 0.354750 & $\mathrm{~F}$ & 3.784760 & -2.150360 & 0.899360 \\
\hline $\mathrm{F}$ & 7.695780 & -0.743490 & 0.159450 & $\mathrm{~F}$ & 5.912670 & -3.758410 & 1.423470 \\
\hline $\mathrm{F}$ & 8.850130 & -1.120190 & -2.298430 & $\mathrm{~F}$ & 7.163210 & -5.079260 & -0.630810 \\
\hline $\mathrm{F}$ & 7.301150 & -1.120440 & -4.555470 & $\mathrm{~F}$ & 6.256490 & -4.795480 & -3.197420 \\
\hline $\mathrm{F}$ & 4.613500 & -0.749730 & -4.361240 & $\mathrm{~F}$ & 4.106020 & -3.211540 & -3.718400 \\
\hline C & 0.922480 & 5.862780 & -2.557400 & C & 3.730160 & 4.647200 & -3.084020 \\
\hline C & 1.103340 & 7.251890 & -2.442040 & C & 4.551550 & 5.787160 & -3.072930 \\
\hline C & 0.918090 & 7.874190 & -1.195820 & C & 4.601280 & 6.594730 & -1.924140 \\
\hline C & 0.552310 & 7.108030 & -0.075570 & C & 3.836430 & 6.257880 & -0.794100 \\
\hline C & 0.377040 & 5.722920 & -0.218730 & C & 3.027130 & 5.111280 & -0.829290 \\
\hline $\mathrm{F}$ & 1.104630 & 5.298450 & -3.754780 & $\mathrm{~F}$ & 3.701420 & 3.899390 & -4.191710 \\
\hline $\mathrm{F}$ & 1.445920 & 7.982510 & -3.504860 & $\mathrm{~F}$ & 5.279210 & 6.111110 & -4.144310 \\
\hline $\mathrm{F}$ & 1.087920 & 9.190850 & -1.075870 & $\mathrm{~F}$ & 5.374530 & 7.680300 & -1.906760 \\
\hline $\mathrm{F}$ & 0.381570 & 7.697750 & 1.110570 & $\mathrm{~F}$ & 3.887360 & 7.022510 & 0.298710 \\
\hline $\mathrm{F}$ & 0.033800 & 5.013530 & 0.868320 & $\mathrm{~F}$ & 2.316830 & 4.805540 & 0.264820 \\
\hline C & -0.065970 & 2.199770 & 5.414670 & C & -3.249010 & -0.969640 & 2.989330 \\
\hline $\mathrm{H}$ & -0.511500 & 2.820410 & 6.224690 & $\mathrm{H}$ & -4.025270 & -1.223160 & 3.746560 \\
\hline $\mathrm{H}$ & 0.389150 & 1.296580 & 5.873650 & $\mathrm{H}$ & -3.538780 & -0.004200 & 2.517930 \\
\hline $\mathrm{H}$ & 0.738630 & 2.797290 & 4.931630 & $\mathrm{H}$ & -3.277370 & -1.763210 & 2.210870 \\
\hline C & -2.153180 & 3.222350 & 3.328190 & C & -1.678760 & 0.366860 & 5.274370 \\
\hline $\mathrm{H}$ & -2.636770 & 3.860500 & 4.102150 & $\mathrm{H}$ & -1.950020 & 1.381730 & 4.908930 \\
\hline $\mathrm{H}$ & -1.404570 & 3.849830 & 2.796460 & $\mathrm{H}$ & -2.490760 & 0.025470 & 5.955930 \\
\hline $\mathrm{H}$ & -2.942860 & 2.925810 & 2.602770 & $\mathrm{H}$ & -0.747240 & 0.453480 & 5.869500 \\
\hline C & -2.732870 & 0.589270 & 4.860760 & C & -0.945570 & -2.570270 & 4.306930 \\
\hline $\mathrm{H}$ & -3.278010 & 1.105280 & 5.683190 & $\mathrm{H}$ & -0.941850 & -3.228460 & 3.409800 \\
\hline $\mathrm{H}$ & -3.471200 & 0.335220 & 4.068260 & $\mathrm{H}$ & 0.078830 & -2.552090 & 4.735230 \\
\hline $\mathrm{H}$ & -2.295470 & -0.349150 & 5.262650 & $\mathrm{H}$ & -1.628560 & -3.028690 & 5.057670 \\
\hline $\mathrm{Si}$ & -1.391730 & 1.713950 & 4.171840 & $\mathrm{Si}$ & -1.569530 & -0.856100 & 3.848040 \\
\hline
\end{tabular}




\section{trans- $\alpha$}

$E_{P B E / S V P}=-6372.072215141$ Hartree $\mathrm{NImag}=0$

\begin{tabular}{|c|c|c|c|}
\hline C & -3.350980 & -0.520260 & 4.595750 \\
\hline C & -4.718280 & -0.785340 & 4.343150 \\
\hline C & -5.617770 & -0.924050 & 5.408030 \\
\hline C & -5.163160 & -0.800510 & 6.732060 \\
\hline C & -3.802380 & -0.535870 & 6.989000 \\
\hline C & -2.897410 & -0.395790 & 5.933240 \\
\hline$N$ & -2.545750 & -0.402980 & 3.449770 \\
\hline $\mathrm{N}$ & -1.307970 & -0.163590 & 3.647800 \\
\hline $\mathrm{C}$ & -0.572660 & -0.063030 & 2.485940 \\
\hline C & -0.898240 & -0.176310 & 1.131270 \\
\hline $\mathrm{N}$ & 0.799990 & 0.206170 & 2.550310 \\
\hline$N$ & 0.231090 & 0.016290 & 0.391010 \\
\hline $\mathrm{C}$ & 1.229290 & 0.242450 & 1.258950 \\
\hline $\mathrm{H}$ & -5.041380 & -0.877870 & 3.293830 \\
\hline $\mathrm{H}$ & -6.681380 & -1.130870 & 5.205370 \\
\hline $\mathrm{H}$ & -5.869610 & -0.910750 & 7.571270 \\
\hline $\mathrm{H}$ & -3.450400 & -0.439450 & 8.029700 \\
\hline $\mathrm{H}$ & -1.831830 & -0.189970 & 6.117700 \\
\hline $\mathrm{H}$ & -1.879850 & -0.384310 & 0.692980 \\
\hline $\mathrm{H}$ & 2.269130 & 0.436800 & 0.965 \\
\hline $\mathrm{C}$ & 1.441370 & 2.854730 & -1.923920 \\
\hline$N$ & 1.688960 & 1.501630 & -1.862270 \\
\hline C & 2.690630 & 3.595060 & -1.952000 \\
\hline C & 3.697920 & 2.667090 & -1.901060 \\
\hline C & 3.058370 & 1.364040 & -1.839820 \\
\hline C & 0.163810 & 3.457040 & -1.940030 \\
\hline C & -1.062750 & 2.758530 & -1.907840 \\
\hline C & -2.366170 & 3.401580 & -1.897640 \\
\hline C & -3.296870 & 2.398670 & -1.833690 \\
\hline C & -2.558140 & 1.147280 & -1.835540 \\
\hline$N$ & -1.204120 & 1.390010 & -1.880200 \\
\hline $\mathrm{Ni}$ & 0.301310 & 0.003430 & -1.639280 \\
\hline$N$ & 1.806790 & -1.383120 & -1.851710 \\
\hline $\mathrm{C}$ & 1.665600 & -2.751220 & -1.896280 \\
\hline C & 2.968990 & -3.393810 & -1.887730 \\
\hline C & 3.899940 & -2.391160 & -1.817240 \\
\hline C & 3.161060 & -1.140210 & -1.808040 \\
\hline C & -3.157100 & -0.132190 & -1.809720 \\
\hline C & -2.453620 & -1.355920 & -1.882550 \\
\hline C & -3.093760 & -2.658570 & -1.947710 \\
\hline $\mathrm{C}$ & -2.087270 & -3.586450 & -1.997940 \\
\hline C & -0.837450 & -2.846380 & -1.960790 \\
\hline $\mathrm{N}$ & -1.084100 & -1.493160 & -1.903390 \\
\hline$C$ & 0.439460 & -3.449710 & -1.954800 \\
\hline $\mathrm{C}$ & 3.760530 & 0.138490 & -1.782130 \\
\hline $\mathrm{H}$ & -4.388270 & 2.500050 & -1.801060 \\
\hline $\mathrm{H}$ & -2.547520 & 4.482610 & -1.936960 \\
\hline $\mathrm{H}$ & -4.175440 & -2.839490 & -1.96953 \\
\hline $\mathrm{H}$ & -2.184500 & -4.677500 & -2.051520 \\
\hline $\mathrm{H}$ & 4.991610 & -2.492440 & -1.78834 \\
\hline $\mathrm{H}$ & 3.150600 & -4.474310 & -1.93863 \\
\hline $\mathrm{H}$ & 2.787550 & 4.686150 & -2.00692 \\
\hline $\mathrm{H}$ & 4.779330 & 2.849790 & -1.92227 \\
\hline
\end{tabular}

\section{trans- $\beta$}

$E_{P B E / S V P}=-6372.072244543$ Hartree NImag $=0$

\begin{tabular}{|c|c|c|c|}
\hline C & -3.273500 & -0.533720 & 4.970820 \\
\hline C & -3.075930 & -0.356960 & 6.359610 \\
\hline C & -4.137270 & -0.549840 & 7.254370 \\
\hline C & -5.403560 & -0.921150 & 6.772180 \\
\hline C & -5.605000 & -1.097980 & 5.388290 \\
\hline C & -4.552490 & -0.907010 & 4.488900 \\
\hline $\mathrm{N}$ & -2.145850 & -0.324850 & 4.145860 \\
\hline $\mathrm{N}$ & -2.354710 & -0.471940 & 2.896470 \\
\hline C & -1.275930 & -0.287900 & 2.077990 \\
\hline C & -1.307560 & -0.416090 & 0.684990 \\
\hline $\mathrm{N}$ & 0.051930 & 0.043110 & 2.424500 \\
\hline $\mathrm{N}$ & -0.061110 & -0.182940 & 0.184580 \\
\hline C & 0.720800 & 0.083590 & 1.240130 \\
\hline $\mathrm{H}$ & -2.075530 & -0.065740 & 6.717710 \\
\hline $\mathrm{H}$ & -3.976190 & -0.410810 & 8.335970 \\
\hline $\mathrm{H}$ & -6.239020 & -1.074780 & 7.474750 \\
\hline $\mathrm{H}$ & -6.599660 & -1.389850 & 5.012030 \\
\hline $\mathrm{H}$ & -4.685040 & -1.040240 & 3.404720 \\
\hline $\mathrm{H}$ & -2.174570 & -0.648510 & 0.054940 \\
\hline $\mathrm{H}$ & 1.789480 & 0.314170 & 1.156080 \\
\hline C & 2.109950 & 2.527490 & -1.033610 \\
\hline $\mathrm{N}$ & 2.132060 & 1.189490 & -1.352080 \\
\hline C & 3.438850 & 2.980960 & -0.663310 \\
\hline C & 4.264430 & 1.889190 & -0.754320 \\
\hline C & 3.430930 & 0.773110 & -1.163550 \\
\hline C & 0.967890 & 3.359750 & -1.061370 \\
\hline C & -0.302580 & 2.979600 & -1.545960 \\
\hline C & -1.452410 & 3.865210 & -1.616050 \\
\hline C & -2.482600 & 3.126950 & -2.135570 \\
\hline C & -1.961430 & 1.789160 & -2.362970 \\
\hline$N$ & -0.633230 & 1.727520 & -2.012370 \\
\hline $\mathrm{Ni}$ & 0.493180 & 0.017280 & -1.767090 \\
\hline $\mathrm{N}$ & 1.743970 & -1.601810 & -1.964470 \\
\hline C & 1.368330 & -2.890390 & -2.264070 \\
\hline C & 2.510180 & -3.784670 & -2.172730 \\
\hline C & 3.582430 & -3.014380 & -1.807430 \\
\hline C & 3.089890 & -1.654150 & -1.678960 \\
\hline C & -2.739050 & 0.701720 & -2.821460 \\
\hline C & -2.278830 & -0.627920 & -2.950950 \\
\hline C & -3.099940 & -1.732140 & -3.416580 \\
\hline C & -2.323880 & -2.858110 & -3.338830 \\
\hline C & -1.023610 & -2.433580 & -2.850190 \\
\hline$N$ & -1.017140 & -1.074460 & -2.626160 \\
\hline C & 0.072600 & -3.301320 & -2.649960 \\
\hline C & 3.890750 & -0.555060 & -1.301260 \\
\hline $\mathrm{H}$ & -3.510090 & 3.456570 & -2.331100 \\
\hline $\mathrm{H}$ & -1.472270 & 4.917920 & -1.309250 \\
\hline $\mathrm{H}$ & -4.136490 & -1.655490 & -3.766890 \\
\hline $\mathrm{H}$ & -2.606430 & -3.884880 & -3.599820 \\
\hline $\mathrm{H}$ & 4.617990 & -3.337850 & -1.646350 \\
\hline $\mathrm{H}$ & 2.497810 & -4.861180 & -2.382300 \\
\hline $\mathrm{H}$ & 3.712380 & 4.009910 & -0.398780 \\
\hline $\mathrm{H}$ & 5.345260 & 1.846650 & -0.571780 \\
\hline
\end{tabular}




\begin{tabular}{|c|c|c|c|c|c|c|c|}
\hline C & 0.109320 & 4.950690 & -1.981720 & C & 1.115750 & 4.724750 & -0.473350 \\
\hline C & 5.249190 & 0.211240 & -1.677260 & C & 5.321640 & -0.823210 & -0.960630 \\
\hline C & -4.641570 & -0.206270 & -1.651240 & C & -4.179170 & 0.964110 & -3.119680 \\
\hline$C$ & 0.495120 & -4.942860 & -1.999740 & C & -0.144780 & -4.766910 & -2.847420 \\
\hline c & 0.862570 & -5.695670 & -0.867700 & C & -0.160170 & -5.637520 & -1.739690 \\
\hline C & 0.907630 & -7.099660 & -0.894520 & C & -0.345650 & -7.021740 & -1.885590 \\
\hline C & 0.580230 & -7.779700 & -2.079770 & C & -0.522450 & -7.562980 & -3.170170 \\
\hline C & 0.210820 & -7.055350 & -3.225710 & C & -0.513030 & -6.719410 & -4.293990 \\
\hline C & 0.173580 & -5.651450 & -3.173860 & C & -0.325160 & -5.337270 & -4.121990 \\
\hline $\mathrm{F}$ & 1.176130 & -5.074570 & 0.275680 & $\mathrm{~F}$ & 0.005080 & -5.150140 & -0.505180 \\
\hline $\mathrm{F}$ & 1.252890 & -7.791930 & 0.194470 & $\mathrm{~F}$ & -0.357810 & -7.821840 & -0.816550 \\
\hline $\mathrm{F}$ & 0.619650 & -9.112140 & -2.116520 & $\mathrm{~F}$ & -0.698490 & -8.875860 & -3.323000 \\
\hline $\mathrm{F}$ & -0.096730 & -7.705030 & -4.351250 & $\mathrm{~F}$ & -0.676180 & -7.236940 & -5.514120 \\
\hline $\mathrm{F}$ & -0.180590 & -4.986540 & -4.278370 & $\mathrm{~F}$ & -0.316830 & -4.561760 & -5.211100 \\
\hline C & -5.207390 & -0.649420 & -0.439410 & C & -5.191540 & 0.474300 & -2.271210 \\
\hline C & -6.595300 & -0.729910 & -0.248790 & C & -6.551660 & 0.722070 & -2.516450 \\
\hline C & -7.457960 & -0.364060 & -1.296040 & C & -6.922400 & 1.481480 & -3.639700 \\
\hline C & -6.924520 & 0.082070 & -2.517800 & C & -5.934920 & 1.984580 & -4.503770 \\
\hline C & -5.530410 & 0.155860 & -2.681590 & C & -4.580710 & 1.722000 & -4.236640 \\
\hline $\mathrm{F}$ & -4.415450 & -0.997810 & 0.585060 & $\mathrm{~F}$ & -4.868390 & -0.243720 & -1.186620 \\
\hline $\mathrm{F}$ & -7.095140 & -1.145770 & 0.920130 & $\mathrm{~F}$ & -7.488880 & 0.249800 & -1.690430 \\
\hline $\mathrm{F}$ & -8.778970 & -0.436910 & -1.130860 & $\mathrm{~F}$ & -8.209860 & 1.725690 & -3.885070 \\
\hline $\mathrm{F}$ & -7.742730 & 0.426990 & -3.514960 & $\mathrm{~F}$ & -6.288690 & 2.703910 & -5.571160 \\
\hline $\mathrm{F}$ & -5.056590 & 0.585030 & -3.855380 & $\mathrm{~F}$ & -3.665450 & 2.214470 & -5.076730 \\
\hline C & 5.862990 & 0.655570 & -0.490730 & C & 5.741280 & -0.862640 & 0.382150 \\
\hline C & 7.256820 & 0.757320 & -0.361450 & C & 7.076590 & -1.108600 & 0.738870 \\
\hline C & 8.075390 & 0.400260 & -1.446840 & C & 8.028190 & -1.326290 & -0.272320 \\
\hline C & 7.494200 & -0.052910 & -2.643550 & C & 7.639800 & -1.292580 & -1.622460 \\
\hline C & 6.095250 & -0.142160 & -2.746290 & C & 6.295440 & -1.046860 & -1.951760 \\
\hline $\mathrm{F}$ & 5.107280 & 0.993170 & 0.568080 & $\mathrm{~F}$ & 4.849140 & -0.665950 & 1.365280 \\
\hline $\mathrm{F}$ & 7.805730 & 1.183750 & 0.779410 & $\mathrm{~F}$ & 7.445660 & -1.142240 & 2.021970 \\
\hline $\mathrm{F}$ & 9.401170 & 0.489980 & -1.340220 & $\mathrm{~F}$ & 9.299860 & -1.564570 & 0.048790 \\
\hline $\mathrm{F}$ & 8.271400 & -0.389090 & -3.675120 & $\mathrm{~F}$ & 8.549210 & -1.492020 & -2.578840 \\
\hline $\mathrm{F}$ & 5.576090 & -0.572810 & -3.899730 & $\mathrm{~F}$ & 5.952670 & -1.026800 & -3.242960 \\
\hline C & 0.429190 & 5.660400 & -3.155410 & C & 1.116980 & 5.895290 & -1.255060 \\
\hline C & 0.392920 & 7.064450 & -3.205210 & C & 1.260200 & 7.168770 & -0.677360 \\
\hline C & 0.025870 & 7.787360 & -2.057580 & C & 1.405580 & 7.287630 & 0.715430 \\
\hline C & -0.299480 & 7.105950 & -0.872520 & C & 1.404100 & 6.136740 & 1.522350 \\
\hline C & -0.254410 & 5.701840 & -0.847610 & C & 1.261270 & 4.878220 & 0.919230 \\
\hline $\mathrm{F}$ & 0.781970 & 4.996440 & -4.260830 & $\mathrm{~F}$ & 0.984190 & 5.814530 & -2.582310 \\
\hline $\mathrm{F}$ & 0.699930 & 7.715600 & -4.330000 & $\mathrm{~F}$ & 1.264240 & 8.263570 & -1.440120 \\
\hline $\mathrm{F}$ & -0.012600 & 9.119890 & -2.092360 & $\mathrm{~F}$ & 1.544360 & 8.491270 & 1.270860 \\
\hline $\mathrm{F}$ & -0.642190 & 7.797050 & 0.218070 & $\mathrm{~F}$ & 1.531730 & 6.247380 & 2.847550 \\
\hline $\mathrm{F}$ & -0.564150 & 5.078850 & 0.295880 & $\mathrm{~F}$ & 1.256870 & 3.795260 & 1.716430 \\
\hline C & 1.702720 & -1.111180 & 5.095650 & C & 0.887020 & -1.027790 & 5.169720 \\
\hline $\mathrm{H}$ & 2.303450 & -1.013240 & 6.027690 & $\mathrm{H}$ & -0.128940 & -1.371040 & 5.451330 \\
\hline $\mathrm{H}$ & 0.650650 & -1.333300 & 5.376440 & $\mathrm{H}$ & 1.418510 & -1.864330 & 4.663970 \\
\hline $\mathrm{H}$ & 2.100680 & -1.976720 & 4.521370 & $\mathrm{H}$ & 1.451930 & -0.783400 & 6.098140 \\
\hline C & 1.070760 & 1.969650 & 4.968280 & C & 0.024210 & 2.042730 & 4.732180 \\
\hline $\mathrm{H}$ & 1.651570 & 2.191920 & 5.891930 & $\mathrm{H}$ & 0.536900 & 2.342510 & 5.674580 \\
\hline $\mathrm{H}$ & 1.102390 & 2.872410 & 4.319140 & $\mathrm{H}$ & 0.143080 & 2.876700 & 4.005120 \\
\hline $\mathrm{H}$ & 0.013360 & 1.782640 & 5.254280 & $\mathrm{H}$ & -1.053520 & 1.897710 & 4.947930 \\
\hline C & 3.547000 & 0.812170 & 3.474850 & C & 2.618380 & 0.940430 & 3.580340 \\
\hline $\mathrm{H}$ & 3.972620 & -0.038390 & 2.899500 & $\mathrm{H}$ & 3.188320 & 0.106820 & 3.116290 \\
\hline $\mathrm{H}$ & 3.607430 & 1.721250 & 2.837700 & $\mathrm{H}$ & 2.662280 & 1.820110 & 2.900990 \\
\hline $\mathrm{H}$ & 4.207580 & 0.979990 & 4.355470 & $\mathrm{H}$ & 3.154270 & 1.222350 & 4.515650 \\
\hline $\mathrm{Si}$ & 1.795510 & 0.473780 & 4.084320 & $\mathrm{Si}$ & 0.841750 & 0.487540 & 4.050990 \\
\hline
\end{tabular}




\section{I.6.5 1-tert-Butyl-5-phenylazoimidazole $\cdot$ NiTPPF $_{20}$ (5)}

\section{cis- $\alpha$}

$E_{P B E / S V P}=-6120.773007405$ Hartree

NImag $=0$

\begin{tabular}{|c|c|c|c|}
\hline C & 1.981090 & -2.701110 & 3.287340 \\
\hline$C$ & 2.096110 & -3.985150 & 2.711460 \\
\hline$C$ & 3.241030 & -4.314730 & 1.971740 \\
\hline $\mathrm{C}$ & 4.293480 & -3.392510 & 1.836780 \\
\hline$C$ & 4.193480 & -2.126560 & 2.441320 \\
\hline$C$ & 3.041720 & -1.774020 & 3.156530 \\
\hline $\mathrm{N}$ & 0.871820 & -2.424370 & 4.132600 \\
\hline $\mathrm{N}$ & 0.069360 & -1.467240 & 3.942800 \\
\hline C & 0.017000 & -0.692690 & 2.787970 \\
\hline C & 0.405830 & -0.875820 & 1.449780 \\
\hline $\mathrm{N}$ & -0.782550 & 0.468170 & 2.790630 \\
\hline $\mathrm{N}$ & -0.131110 & 0.114230 & 0.686660 \\
\hline C & -0.820660 & 0.911780 & 1.513690 \\
\hline $\mathrm{H}$ & 1.271190 & -4.704090 & 2.833870 \\
\hline $\mathrm{H}$ & 3.317140 & -5.311240 & 1.506080 \\
\hline $\mathrm{H}$ & 5.197940 & -3.664670 & 1.269060 \\
\hline $\mathrm{H}$ & 5.015860 & -1.399070 & 2.346940 \\
\hline $\mathrm{H}$ & 2.960900 & -0.783290 & 3.631900 \\
\hline $\mathrm{H}$ & 1.002090 & -1.683490 & 1.012940 \\
\hline $\mathrm{H}$ & -1.337620 & 1.819330 & 1.189280 \\
\hline$C$ & 1.139120 & 3.068280 & -1.4714 \\
\hline $\mathrm{N}$ & 1.297620 & 1.703090 & -1.540770 \\
\hline$C$ & 2.432480 & 3.730420 & -1.515060 \\
\hline$C$ & 3.375970 & 2.739880 & -1.590260 \\
\hline$C$ & 2.653180 & 1.479290 & -1.603160 \\
\hline$C$ & -0.094660 & 3.747370 & -1.368450 \\
\hline$C$ & -1.362250 & 3.125340 & -1.337310 \\
\hline$C$ & -2.614920 & 3.838500 & -1.164090 \\
\hline$C$ & -3.602550 & 2.888290 & -1.108050 \\
\hline$C$ & -2.949450 & 1.600150 & -1.261590 \\
\hline$N$ & -1.589330 & 1.768010 & -1.404590 \\
\hline $\mathrm{Ni}$ & -0.162810 & 0.277760 & -1.344020 \\
\hline $\mathrm{N}$ & 1.226760 & -1.178190 & -1.761810 \\
\hline$C$ & 0.999610 & -2.534130 & -1.845860 \\
\hline$C$ & 2.256240 & -3.251250 & -1.966860 \\
\hline$C$ & 3.249600 & -2.307650 & -1.933170 \\
\hline$C$ & 2.593670 & -1.017900 & -1.799900 \\
\hline$C$ & -3.622550 & 0.357750 & -1.234800 \\
\hline$C$ & -3.005830 & -0.902280 & -1.400530 \\
\hline$C$ & -3.727530 & -2.163780 & -1.437570 \\
\hline $\mathrm{C}$ & -2.786720 & -3.147800 & -1.589300 \\
\hline C & -1.496490 & -2.481200 & -1.644590 \\
\hline $\mathrm{N}$ & -1.655870 & -1.118570 & -1.549030 \\
\hline $\mathrm{C}$ & -0.263840 & -3.157250 & -1.76924 \\
\hline C & 3.274440 & 0.215340 & -1.697430 \\
\hline $\mathrm{H}$ & -4.679340 & 3.051510 & -0.97911 \\
\hline $\mathrm{H}$ & -2.726660 & 4.926880 & -1.085370 \\
\hline $\mathrm{H}$ & -4.816540 & -2.280130 & -1.375820 \\
\hline $\mathrm{H}$ & -2.951020 & -4.229280 & -1.67226 \\
\hline $\mathrm{H}$ & 4.331880 & -2.473980 & -1.990520 \\
\hline $\mathrm{H}$ & 2.365510 & -4.338660 & -2.06008 \\
\hline $\mathrm{H}$ & 2.596280 & 4.815070 & -1.50610 \\
\hline $\mathrm{H}$ & 4.465310 & 2.851900 & -1.65658 \\
\hline
\end{tabular}

cis- $\beta$

$E_{P B E / S V P}=-6120.758361326$ Hartree NImag $=0$

\begin{tabular}{|c|c|c|c|}
\hline $\mathrm{C}$ & -1.463540 & -0.914240 & 4.889300 \\
\hline $\mathrm{C}$ & -1.770740 & -0.525130 & 6.215920 \\
\hline C & -2.905180 & 0.248960 & 6.483010 \\
\hline $\mathrm{C}$ & -3.788050 & 0.584410 & 5.440140 \\
\hline$C$ & -3.521510 & 0.149820 & 4.129520 \\
\hline C & -2.356810 & -0.576100 & 3.841170 \\
\hline$N$ & -0.355110 & -1.792540 & 4.754900 \\
\hline $\mathrm{N}$ & 0.417550 & -1.852100 & 3.760920 \\
\hline$C$ & 0.427120 & -0.959760 & 2.673790 \\
\hline$C$ & 0.309540 & -1.386950 & 1.351600 \\
\hline $\mathrm{N}$ & 0.580650 & 0.440170 & 2.621740 \\
\hline $\mathrm{N}$ & 0.306680 & -0.296520 & 0.523930 \\
\hline $\mathrm{C}$ & 0.491020 & 0.770210 & 1.296870 \\
\hline $\mathrm{H}$ & -1.095700 & -0.851640 & 7.023180 \\
\hline $\mathrm{H}$ & -3.120550 & 0.568480 & 7.515840 \\
\hline $\mathrm{H}$ & -4.698620 & 1.167910 & 5.652520 \\
\hline $\mathrm{H}$ & -4.228840 & 0.375620 & 3.315890 \\
\hline $\mathrm{H}$ & -2.165820 & -0.909800 & 2.809860 \\
\hline $\mathrm{H}$ & 0.179780 & -2.414920 & 0.993020 \\
\hline $\mathrm{H}$ & 0.609690 & 1.791670 & 0.919130 \\
\hline $\mathrm{C}$ & 1.740890 & 2.405900 & -1.759490 \\
\hline $\mathrm{N}$ & 1.762090 & 1.029630 & -1.802570 \\
\hline$C$ & 3.088480 & 2.936690 & -1.869130 \\
\hline$C$ & 3.924020 & 1.857360 & -1.986120 \\
\hline$C$ & 3.085340 & 0.672410 & -1.926130 \\
\hline$C$ & 0.582500 & 3.202710 & -1.622710 \\
\hline$C$ & -0.740390 & 2.710340 & -1.566300 \\
\hline$C$ & -1.921230 & 3.551580 & -1.478510 \\
\hline$C$ & -2.999740 & 2.706730 & -1.436380 \\
\hline $\mathrm{C}$ & -2.470340 & 1.355590 & -1.501880 \\
\hline $\mathrm{N}$ & -1.097410 & 1.381770 & -1.589600 \\
\hline $\mathrm{Ni}$ & 0.168320 & -0.242040 & -1.502830 \\
\hline $\mathrm{N}$ & 1.403990 & -1.840250 & -1.876950 \\
\hline$C$ & 1.043730 & -3.168490 & -1.905650 \\
\hline$C$ & 2.221560 & -4.009230 & -2.033750 \\
\hline$C$ & 3.301030 & -3.167480 & -2.066360 \\
\hline $\mathrm{C}$ & 2.777040 & -1.814940 & -1.974270 \\
\hline$C$ & -3.266470 & 0.190530 & -1.468790 \\
\hline $\mathrm{C}$ & -2.779110 & -1.131020 & -1.551120 \\
\hline$C$ & -3.625400 & -2.311580 & -1.542580 \\
\hline$C$ & -2.789100 & -3.392170 & -1.641780 \\
\hline $\mathrm{C}$ & -1.437220 & -2.864430 & -1.719540 \\
\hline $\mathrm{N}$ & -1.455610 & -1.489980 & -1.664400 \\
\hline$C$ & -0.276750 & -3.663470 & -1.826470 \\
\hline $\mathrm{C}$ & 3.577760 & -0.650700 & -1.992770 \\
\hline $\mathrm{H}$ & -4.061200 & 2.975810 & -1.373280 \\
\hline $\mathrm{H}$ & -1.926440 & 4.648490 & -1.467680 \\
\hline $\mathrm{H}$ & -4.720440 & -2.313170 & -1.477370 \\
\hline $\mathrm{H}$ & -3.064670 & -4.453350 & -1.662450 \\
\hline $\mathrm{H}$ & 4.359730 & -3.440690 & -2.148540 \\
\hline $\mathrm{H}$ & 2.222290 & -5.104000 & -2.098780 \\
\hline $\mathrm{H}$ & 3.361360 & 3.998890 & -1.872230 \\
\hline $\mathrm{H}$ & 5.013010 & 1.865530 & -2.114870 \\
\hline
\end{tabular}




\begin{tabular}{|c|c|c|c|c|c|c|c|}
\hline$c$ & -0.052480 & 5.235080 & -1.230940 & C & 0.767600 & 4.679620 & -1.487950 \\
\hline E & 4.766120 & 0.168390 & -1.628430 & C & 5.059050 & -0.825410 & -2.090220 \\
\hline c & -5.091580 & 0.370240 & -0.964170 & C & -4.741700 & 0.371920 & -1.307340 \\
\hline C & -0.296600 & -4.651060 & -1.711820 & C & -0.462860 & -5.146340 & -1.841130 \\
\hline C & -0.201560 & -5.456690 & -2.861040 & C & -0.108790 & -5.934560 & -0.729430 \\
\hline C & -0.251430 & -6.859890 & -2.783730 & C & -0.290110 & -7.327510 & -0.721700 \\
\hline C & -0.387480 & -7.480970 & -1.530270 & C & -0.840050 & -7.958890 & -1.850900 \\
\hline C & -0.473330 & -6.699900 & -0.364630 & C & -1.205000 & -7.197510 & -2.974290 \\
\hline C & -0.430740 & -5.300860 & -0.470320 & C & -1.009850 & -5.806150 & -2.958460 \\
\hline $\mathrm{F}$ & -0.063380 & -4.892620 & -4.064720 & $\mathrm{~F}$ & 0.407860 & -5.357220 & 0.363860 \\
\hline $\mathrm{F}$ & -0.176690 & -7.604680 & -3.889060 & $\mathrm{~F}$ & 0.048460 & -8.053060 & 0.346340 \\
\hline $\mathrm{F}$ & -0.431880 & -8.810780 & -1.446620 & $\mathrm{~F}$ & -1.018600 & -9.280120 & -1.854160 \\
\hline $\mathrm{F}$ & -0.593960 & -7.288000 & 0.828900 & $\mathrm{~F}$ & -1.729110 & -7.798720 & -4.045200 \\
\hline $\mathrm{F}$ & -0.511210 & -4.578110 & 0.655950 & $\mathrm{~F}$ & -1.362870 & -5.105220 & -4.040580 \\
\hline C & -5.595430 & -0.094530 & 0.266780 & C & -5.357200 & 0.181090 & -0.057800 \\
\hline C & -6.969010 & -0.093890 & 0.556710 & C & -6.735170 & 0.367470 & 0.131610 \\
\hline C & -7.875380 & 0.389380 & -0.402720 & C & -7.534530 & 0.741150 & -0.962460 \\
\hline C & -7.403010 & 0.865180 & -1.637790 & C & -6.949520 & 0.932370 & -2.226160 \\
\hline C & -6.023440 & 0.847660 & -1.905650 & C & -5.565060 & 0.748060 & -2.384530 \\
\hline $\mathrm{F}$ & -4.751710 & -0.548990 & 1.206160 & $\mathrm{~F}$ & -4.615890 & -0.178690 & 1.002280 \\
\hline $\mathrm{F}$ & -7.418400 & -0.540030 & 1.732250 & $\mathrm{~F}$ & -7.285870 & 0.194780 & 1.336790 \\
\hline $\mathrm{F}$ & -9.182030 & 0.398790 & -0.139110 & $\mathrm{~F}$ & -8.846100 & 0.916830 & -0.800890 \\
\hline $\mathrm{F}$ & -8.266090 & 1.325770 & -2.545780 & $\mathrm{~F}$ & -7.710150 & 1.284040 & -3.264760 \\
\hline $\mathrm{F}$ & -5.603570 & 1.302230 & -3.090360 & $\mathrm{~F}$ & -5.034100 & 0.942530 & -3.594660 \\
\hline C & 5.424170 & 0.381980 & -0.402300 & C & 5.905220 & -0.503210 & -1.010480 \\
\hline C & 6.817430 & 0.271710 & -0.276040 & C & 7.299860 & -0.648430 & -1.090530 \\
\hline C & 7.591050 & -0.036010 & -1.408000 & C & 7.878780 & -1.132760 & -2.275980 \\
\hline C & 6.964060 & -0.238770 & -2.649510 & C & 7.060960 & -1.465590 & -3.369080 \\
\hline C & 5.565640 & -0.132960 & -2.746860 & C & 5.668880 & -1.306240 & -3.265980 \\
\hline $\mathrm{F}$ & 4.714990 & 0.670560 & 0.697180 & $\mathrm{~F}$ & 5.387800 & -0.042470 & 0.135380 \\
\hline $\mathrm{F}$ & 7.403510 & 0.442370 & 0.913340 & $\mathrm{~F}$ & 8.075040 & -0.333870 & -0.049330 \\
\hline $\mathrm{F}$ & 8.915880 & -0.141120 & -1.303890 & $\mathrm{~F}$ & 9.201430 & -1.275620 & -2.363020 \\
\hline $\mathrm{F}$ & 7.698800 & -0.532990 & -3.724390 & $\mathrm{~F}$ & 7.608700 & -1.923070 & -4.497510 \\
\hline $\mathrm{F}$ & 4.997880 & -0.341660 & -3.938890 & $\mathrm{~F}$ & 4.921010 & -1.623760 & -4.327590 \\
\hline C & -0.421030 & 6.087280 & -2.289240 & C & 1.106640 & 5.494650 & -2.584140 \\
\hline C & -0.365990 & 7.486120 & -2.165100 & C & 1.278150 & 6.883040 & -2.447630 \\
\hline C & 0.060940 & 8.058090 & -0.954270 & C & 1.104920 & 7.483080 & -1.188780 \\
\hline C & 0.426030 & 7.231590 & 0.122580 & C & 0.768740 & 6.693940 & -0.075070 \\
\hline C & 0.360890 & 5.836770 & -0.026670 & C & 0.605480 & 5.310220 & -0.240770 \\
\hline $\mathrm{F}$ & -0.834750 & 5.572680 & -3.451070 & $\mathrm{~F}$ & 1.275910 & 4.952490 & -3.793070 \\
\hline $\mathrm{F}$ & -0.712200 & 8.271430 & -3.187380 & $\mathrm{~F}$ & 1.599200 & 7.633560 & -3.502910 \\
\hline $\mathrm{F}$ & 0.117490 & 9.383850 & -0.825970 & $\mathrm{~F}$ & 1.263400 & 8.799080 & -1.049100 \\
\hline $\mathrm{F}$ & 0.825220 & 7.774620 & 1.275990 & $\mathrm{~F}$ & 0.612300 & 7.261540 & 1.123600 \\
\hline $\mathrm{F}$ & 0.699110 & 5.072660 & 1.021400 & $\mathrm{~F}$ & 0.297390 & 4.576490 & 0.845450 \\
\hline C & -1.501090 & 1.090250 & 3.955920 & C & 1.055620 & 1.438030 & 3.656880 \\
\hline c & -2.102610 & 2.436730 & 3.514830 & C & 2.196310 & 2.273520 & 3.028930 \\
\hline H & -2.594030 & 2.902560 & 4.394550 & $\mathrm{H}$ & 2.637200 & 2.925850 & 3.812650 \\
\hline H & -1.325530 & 3.143530 & 3.148740 & $\mathrm{H}$ & 3.000740 & 1.619190 & 2.628580 \\
\hline H & -2.877890 & 2.311750 & 2.728480 & $\mathrm{H}$ & 1.839140 & 2.939930 & 2.216750 \\
\hline C & -0.493770 & 1.333480 & 5.096740 & C & 1.623130 & 0.716280 & 4.890160 \\
\hline $\mathrm{H}$ & -1.012320 & 1.833640 & 5.942730 & $\mathrm{H}$ & 2.393370 & -0.033370 & 4.609590 \\
\hline $\mathrm{H}$ & -0.068480 & 0.377100 & 5.463950 & $\mathrm{H}$ & 2.105800 & 1.475270 & 5.541350 \\
\hline $\mathrm{H}$ & 0.333940 & 1.994680 & 4.758600 & $\mathrm{H}$ & 0.842320 & 0.215140 & 5.493100 \\
\hline C & -2.630620 & 0.133980 & 4.391060 & C & -0.118480 & 2.357560 & 4.041920 \\
\hline $\mathrm{H}$ & -3.344810 & -0.033500 & 3.554920 & $\mathrm{H}$ & -0.498200 & 2.906560 & 3.153160 \\
\hline $\mathrm{H}$ & -2.213220 & -0.842190 & 4.711830 & $\mathrm{H}$ & -0.956870 & 1.783930 & 4.488530 \\
\hline $\mathrm{H}$ & -3.187830 & 0.581790 & 5.242520 & $\mathrm{H}$ & 0.226510 & 3.108080 & 4.785720 \\
\hline
\end{tabular}




\section{trans- $\alpha$}

$E_{P B E / S V P}=-6120.794544253$ Hartree $\mathrm{NImag}=0$

H

H

$\mathrm{N}$

C

C

$$
-3.337320
$$

$-0.399320$

$-0.525230$

$-0.648090$

4.615840

4.341640

5.392240

$-0.649170$

6.723110

7.001400

$-0.400570 \quad 5.960290$

$-0.287200 \quad 3.482840$

$-0.146910 \quad 3.704100$

$-0.054970 \quad 2.549160$

$-0.106470 \quad 1.197730$

$0.117450 \quad 2.572760$

$0.026460 \quad 0.433190$

$0.159330 \quad 1.274710$

$-0.522580 \quad 3.287650$

$-0.745390 \quad 5.172900$

$-0.748340 \quad 7.551080$

$-0.528090 \quad 8.047370$

$-0.304820 \quad 6.161840$

$-0.232770 \quad 0.784390$

$0.287750 \quad 0.952920$

$2.829070-1.834030$

$1.465440-1.799590$

$3.513480-1.830780$

$2.540130-1.792960$

$1.266410-1.770670$

$3.486360-1.862360$

$2.839750-1.866840$

$3.537910-1.895810$

$2.575860-1.859580$

$1.294160-1.832230$

$1.478390-1.842360$

$0.026430-1.595190$

$-1.424780-1.809370$

$-2.785690-1.853230$

$-3.483370-1.871500$

$-2.521850-1.815840$

$-1.240500-1.784430$

$0.043870-1.800890$

$-1.211570-1.835340$

$-2.484600-1.864540$

$-3.458180-1.894610$

$-2.774210-1.882510$

$-1.410640-1.853540$

$-3.432280-1.883580$

$0.009460-1.742880$

$2.722740-1.861920$

$4.625620-1.942740$

$-2.615310-1.874720$

$-4.544950-1.919790$

$-2.669310-1.808920$

$-4.570400-1.930000$

$4.600350-1.858300$

$2.673270-1.797500$

\section{trans- $\beta$}

$E_{P B E / S V P}=-6120.788005657$ Hartree NImag $=0$

\begin{tabular}{|c|c|c|c|}
\hline $\mathrm{C}$ & -3.473640 & -0.972920 & 4.773430 \\
\hline$C$ & -3.486630 & -1.040060 & 6.184980 \\
\hline$c$ & -4.675670 & -1.330100 & 6.867440 \\
\hline C & -5.861210 & -1.553230 & 6.147000 \\
\hline 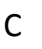 & -5.853030 & -1.484020 & 4.739050 \\
\hline$C$ & -4.671080 & -1.196380 & 4.050560 \\
\hline $\mathrm{N}$ & -2.223690 & -0.680230 & 4.179520 \\
\hline$N$ & -2.233450 & -0.662670 & 2.906900 \\
\hline$C$ & -1.103880 & -0.403650 & 2.179840 \\
\hline C & -1.159010 & -0.433420 & 0.777730 \\
\hline$N$ & 0.255390 & -0.097710 & 2.490550 \\
\hline $\mathrm{N}$ & 0.064450 & -0.174220 & 0.252660 \\
\hline$C$ & 0.884620 & 0.019160 & 1.294170 \\
\hline $\mathrm{H}$ & -2.543720 & -0.861850 & 6.726480 \\
\hline $\mathrm{H}$ & -4.678960 & -1.383120 & 7.968410 \\
\hline $\mathrm{H}$ & -6.797880 & -1.781460 & 6.681740 \\
\hline $\mathrm{H}$ & -6.785500 & -1.657770 & 4.176410 \\
\hline $\mathrm{H}$ & -4.640300 & -1.137180 & 2.952120 \\
\hline $\mathrm{H}$ & -2.050350 & -0.624480 & 0.167690 \\
\hline $\mathrm{H}$ & 1.949140 & 0.250410 & 1.184250 \\
\hline$C$ & 2.035650 & 2.739870 & -0.881760 \\
\hline $\mathrm{N}$ & 2.127330 & 1.420200 & -1.264370 \\
\hline$C$ & 3.336230 & 3.236760 & -0.468290 \\
\hline$C$ & 4.217190 & 2.195980 & -0.613020 \\
\hline$C$ & 3.444690 & 1.061240 & -1.086280 \\
\hline$C$ & 0.850590 & 3.509410 & -0.877700 \\
\hline$C$ & -0.403880 & 3.069800 & -1.353270 \\
\hline$C$ & -1.587250 & 3.909910 & -1.421210 \\
\hline$C$ & -2.589200 & 3.131830 & -1.938640 \\
\hline$C$ & -2.013220 & 1.818770 & -2.178150 \\
\hline $\mathrm{N}$ & -0.682600 & 1.809820 & -1.830430 \\
\hline $\mathrm{Ni}$ & 0.547740 & 0.167740 & -1.694590 \\
\hline $\mathrm{N}$ & 1.881300 & -1.368020 & -1.972520 \\
\hline$C$ & 1.566640 & -2.666860 & -2.298350 \\
\hline$C$ & 2.752580 & -3.504360 & -2.240380 \\
\hline$C$ & 3.788380 & -2.690800 & -1.863890 \\
\hline$C$ & 3.229880 & -1.360190 & -1.695950 \\
\hline$C$ & -2.741850 & 0.706030 & -2.655620 \\
\hline$C$ & -2.215830 & -0.592860 & -2.832120 \\
\hline$C$ & -2.987570 & -1.727480 & -3.308850 \\
\hline$C$ & -2.148410 & -2.810160 & -3.285000 \\
\hline$C$ & -0.860710 & -2.327210 & -2.817120 \\
\hline $\mathrm{N}$ & -0.923160 & -0.977250 & -2.553450 \\
\hline$C$ & 0.286760 & -3.137250 & -2.667200 \\
\hline$C$ & 3.973550 & -0.234720 & -1.278140 \\
\hline $\mathrm{H}$ & -3.628650 & 3.421040 & -2.134930 \\
\hline $\mathrm{H}$ & -1.639620 & 4.965000 & -1.125500 \\
\hline $\mathrm{H}$ & -4.036580 & -1.698770 & -3.627940 \\
\hline $\mathrm{H}$ & -2.380130 & -3.843290 & -3.570410 \\
\hline $\mathrm{H}$ & 4.839230 & -2.967950 & -1.716930 \\
\hline $\mathrm{H}$ & 2.790660 & -4.575320 & -2.474350 \\
\hline $\mathrm{H}$ & 3.555840 & 4.255720 & -0.127600 \\
\hline $\mathrm{H}$ & 5.296960 & 2.199820 & -0.420150 \\
\hline
\end{tabular}




\begin{tabular}{|c|c|c|c|c|c|c|c|}
\hline E & 0.327330 & 4.981340 & -1.875800 & C & 0.920870 & 4.876120 & -0.276160 \\
\hline F & 5.256160 & 0.003490 & -1.656530 & C & 5.413950 & -0.444010 & -0.937940 \\
\hline C & -4.642670 & 0.055210 & -1.679350 & C & -4.195430 & 0.908110 & -2.936610 \\
\hline C & 0.284080 & -4.926770 & -1.905010 & C & 0.136830 & -4.608930 & -2.883470 \\
\hline C & 0.647950 & -5.673650 & -0.767700 & C & 0.152750 & -5.489450 & -1.783780 \\
\hline C & 0.650400 & -7.078560 & -0.773420 & C & 0.010280 & -6.877330 & -1.942060 \\
\hline C & 0.284760 & -7.765760 & -1.943260 & C & -0.149290 & -7.411800 & -3.231730 \\
\hline C & -0.082700 & -7.047630 & -3.093750 & C & -0.166890 & -6.558250 & -4.348000 \\
\hline C & -0.079060 & -5.642530 & -3.062490 & C & -0.023440 & -5.172240 & -4.163430 \\
\hline $\mathrm{F}$ & 1.000610 & -5.045120 & 0.360200 & $\mathrm{~F}$ & 0.303040 & -5.007550 & -0.545170 \\
\hline $\mathrm{F}$ & 0.993680 & -7.764820 & 0.319980 & $\mathrm{~F}$ & 0.021680 & -7.686940 & -0.880160 \\
\hline $\mathrm{F}$ & 0.285300 & -9.099130 & -1.960870 & $\mathrm{~F}$ & -0.284560 & -8.728050 & -3.396420 \\
\hline $\mathrm{F}$ & -0.425050 & -7.704840 & -4.204640 & $\mathrm{~F}$ & -0.315380 & -7.069750 & -5.572520 \\
\hline $\mathrm{F}$ & -0.430240 & -4.983620 & -4.171500 & $\mathrm{~F}$ & -0.041970 & -4.385920 & -5.244540 \\
\hline C & -5.261190 & -0.225830 & -0.446380 & C & -5.179820 & 0.383930 & -2.076480 \\
\hline C & -6.654210 & -0.173270 & -0.282540 & C & -6.550130 & 0.588410 & -2.305510 \\
\hline C & -7.465000 & 0.153920 & -1.382870 & C & -6.959590 & 1.334070 & -3.424660 \\
\hline C & -6.877040 & 0.429530 & -2.629770 & C & -5.999970 & 1.867320 & -4.302050 \\
\hline C & -5.478900 & 0.376570 & -2.764730 & C & -4.635080 & 1.648550 & -4.050600 \\
\hline $\mathrm{F}$ & -4.513260 & -0.537670 & 0.622150 & $\mathrm{~F}$ & -4.820400 & -0.324670 & -0.996850 \\
\hline $\mathrm{F}$ & -7.208820 & -0.423340 & 0.908710 & $\mathrm{~F}$ & -7.461760 & 0.087820 & -1.467660 \\
\hline $\mathrm{F}$ & -8.790020 & 0.206490 & -1.243810 & $\mathrm{~F}$ & -8.257540 & 1.536460 & -3.652430 \\
\hline $\mathrm{F}$ & -7.647880 & 0.739530 & -3.674540 & $\mathrm{~F}$ & -6.388500 & 2.574120 & -5.365800 \\
\hline $\mathrm{F}$ & -4.947370 & 0.650220 & -3.960160 & $\mathrm{~F}$ & -3.745220 & 2.168680 & -4.901300 \\
\hline C & 5.910650 & 0.323800 & -0.452590 & C & 5.837400 & -0.458100 & 0.404550 \\
\hline C & 7.310070 & 0.316390 & -0.339070 & C & 7.179800 & -0.659250 & 0.762040 \\
\hline C & 8.088300 & -0.019280 & -1.460530 & C & 8.137390 & -0.855410 & -0.247930 \\
\hline C & 7.464130 & -0.343110 & -2.677440 & C & 7.746320 & -0.845640 & -1.597690 \\
\hline C & 6.061320 & -0.327300 & -2.763100 & C & 6.394920 & -0.642970 & -1.927720 \\
\hline $\mathrm{F}$ & 5.189450 & 0.640070 & 0.636970 & $\mathrm{~F}$ & 4.941820 & -0.278930 & 1.389890 \\
\hline $\mathrm{F}$ & 7.904290 & 0.616450 & 0.819400 & $\mathrm{~F}$ & 7.550130 & -0.670850 & 2.045130 \\
\hline $\mathrm{F}$ & 9.418180 & -0.032210 & -1.368660 & $\mathrm{~F}$ & 9.416120 & -1.050660 & 0.074060 \\
\hline $\mathrm{F}$ & 8.205630 & -0.660710 & -3.740420 & $\mathrm{~F}$ & 8.659960 & -1.026260 & -2.553490 \\
\hline $\mathrm{F}$ & 5.495330 & -0.639040 & -3.932820 & $\mathrm{~F}$ & 6.051960 & -0.642600 & -3.218960 \\
\hline C & 0.688610 & 5.702920 & -3.029930 & C & 1.551260 & 5.954620 & -0.924000 \\
\hline C & 0.690170 & 7.108230 & -3.053920 & C & 1.642920 & 7.223760 & -0.327560 \\
\hline C & 0.325390 & 7.819840 & -1.898490 & C & 1.090240 & 7.432200 & 0.947390 \\
\hline C & -0.038800 & 7.126320 & -0.731900 & C & 0.450020 & 6.374810 & 1.616400 \\
\hline C & -0.033580 & 5.721300 & -0.733280 & C & 0.374850 & 5.115770 & 0.999830 \\
\hline $\mathrm{F}$ & 1.039550 & 5.049580 & -4.142320 & $\mathrm{~F}$ & 2.092630 & 5.784900 & -2.134340 \\
\hline $\mathrm{F}$ & 1.031070 & 7.771780 & -4.161450 & $\mathrm{~F}$ & 2.250870 & 8.229390 & -0.960560 \\
\hline $\mathrm{F}$ & 0.324010 & 9.153290 & -1.909000 & $\mathrm{~F}$ & 1.177020 & 8.631820 & 1.522580 \\
\hline $\mathrm{F}$ & -0.380000 & 7.806710 & 0.365820 & $\mathrm{~F}$ & -0.071540 & 6.569990 & 2.830340 \\
\hline $\mathrm{F}$ & -0.382570 & 5.085530 & 0.391460 & $\mathrm{~F}$ & -0.227310 & 4.120320 & 1.667320 \\
\hline C & 1.762050 & 0.233620 & 3.781400 & C & 0.922340 & 0.166050 & 3.816910 \\
\hline C & 1.640870 & -1.066060 & 4.602500 & C & 0.865960 & -1.115060 & 4.669100 \\
\hline $\mathrm{H}$ & 2.298560 & -1.004130 & 5.496220 & $\mathrm{H}$ & 1.357740 & -0.927720 & 5.648320 \\
\hline H & 0.595460 & -1.222090 & 4.939680 & $\mathrm{H}$ & -0.185930 & -1.413410 & 4.856250 \\
\hline H & 1.956940 & -1.944000 & 3.997810 & $\mathrm{H}$ & 1.405800 & -1.945150 & 4.163000 \\
\hline C & 1.310960 & 1.458790 & 4.602040 & C & 0.197220 & 1.348740 & 4.488220 \\
\hline $\mathrm{H}$ & 1.955060 & 1.558780 & 5.502150 & $\mathrm{H}$ & 0.227920 & 2.247480 & 3.833360 \\
\hline $\mathrm{H}$ & 1.404300 & 2.390090 & 4.001750 & $\mathrm{H}$ & -0.858430 & 1.088500 & 4.709750 \\
\hline $\mathrm{H}$ & 0.256540 & 1.348610 & 4.929360 & $\mathrm{H}$ & 0.702440 & 1.595770 & 5.447190 \\
\hline C & 3.220370 & 0.425590 & 3.333920 & C & 2.395890 & 0.549840 & 3.587330 \\
\hline $\mathrm{H}$ & 3.599040 & -0.436110 & 2.743630 & $\mathrm{H}$ & 2.980040 & -0.261270 & 3.103270 \\
\hline $\mathrm{H}$ & 3.360650 & 1.353500 & 2.738770 & $\mathrm{H}$ & 2.504750 & 1.479570 & 2.988170 \\
\hline H & 3.854100 & 0.512210 & 4.241500 & $\mathrm{H}$ & 2.854910 & 0.739510 & 4.580320 \\
\hline
\end{tabular}




\section{I.6.6 1-Methyl-4'-methoxy-5-phenylazoimidazole $\cdot \mathrm{NiTPPF}_{20}(2 \mathrm{~b})$}

\begin{tabular}{|c|c|c|c|c|c|c|c|}
\hline \multicolumn{4}{|c|}{ cis- $\alpha$} & \multicolumn{4}{|c|}{ cis- $\beta$} \\
\hline \multicolumn{4}{|c|}{$E_{P B E / S V P}=-6117.410019923$ Hartree } & \multicolumn{4}{|c|}{$E_{\mathrm{PBE} / \mathrm{SVP}}=-6117.400704494$ Hartree } \\
\hline \multicolumn{4}{|c|}{ NImag $=0$} & \multicolumn{4}{|c|}{ NImag $=0$} \\
\hline C & 1.369900 & -2.073120 & 3.994380 & $\mathrm{C}$ & 0.734390 & -0.898420 & 4.735050 \\
\hline C & 1.270540 & -3.468890 & 4.232650 & $\mathrm{C}$ & 0.292320 & -1.234940 & 6.036260 \\
\hline C & 2.065060 & -4.372650 & 3.534650 & $\mathrm{C}$ & -0.219750 & -2.502250 & 6.330230 \\
\hline C & 3.033600 & -3.902950 & 2.616340 & $\mathrm{C}$ & -0.235420 & -3.497530 & 5.325160 \\
\hline C & 3.199820 & -2.510610 & 2.430510 & $\mathrm{C}$ & 0.258580 & -3.190070 & 4.032500 \\
\hline C & 2.364590 & -1.611200 & 3.100870 & $\mathrm{C}$ & 0.717680 & -1.909830 & 3.732610 \\
\hline $\mathrm{N}$ & 0.577490 & -1.242230 & 4.814390 & $\mathrm{~N}$ & 1.326900 & 0.374950 & 4.604060 \\
\hline $\mathrm{N}$ & 0.057070 & -0.145630 & 4.454740 & $\mathrm{~N}$ & 1.411350 & 1.039030 & 3.527040 \\
\hline C & -0.072820 & 0.338330 & 3.157780 & $\mathrm{C}$ & 0.687800 & 0.804580 & 2.360220 \\
\hline C & -0.138600 & -0.160240 & 1.843810 & $\mathrm{C}$ & 1.149530 & 0.982530 & 1.052210 \\
\hline $\mathrm{N}$ & -0.512030 & 1.668390 & 3.057910 & $\mathrm{~N}$ & -0.688710 & 0.546810 & 2.237280 \\
\hline $\mathrm{N}$ & -0.586320 & 0.823720 & 1.008510 & $\mathrm{~N}$ & 0.115850 & 0.791330 & 0.175000 \\
\hline C & -0.790370 & 1.915400 & 1.758420 & $\mathrm{C}$ & -0.975570 & 0.559740 & 0.905160 \\
\hline $\mathrm{H}$ & 0.532050 & -3.816440 & 4.972310 & $\mathrm{H}$ & 0.360070 & -0.461150 & 6.817560 \\
\hline $\mathrm{H}$ & 2.508820 & -0.532400 & 2.935560 & $\mathrm{H}$ & 1.105950 & -1.706250 & 2.722610 \\
\hline $\mathrm{H}$ & 0.100030 & -1.165090 & 1.478320 & $\mathrm{H}$ & 2.174310 & 1.201200 & 0.729360 \\
\hline $\mathrm{H}$ & -1.120910 & 2.893360 & 1.384030 & $\mathrm{H}$ & -1.994350 & 0.438270 & 0.512000 \\
\hline C & 0.385620 & 3.637050 & -1.226610 & $\mathrm{C}$ & 1.378680 & 3.571870 & -2.239950 \\
\hline $\mathrm{N}$ & 0.616730 & 2.284510 & -1.334280 & $\mathrm{~N}$ & 1.598070 & 2.215860 & -2.147320 \\
\hline C & 1.642220 & 4.366660 & -1.223550 & $C$ & 2.642640 & 4.285740 & -2.301060 \\
\hline C & 2.636550 & 3.432170 & -1.345970 & $\mathrm{C}$ & 3.630300 & 3.338330 & -2.246960 \\
\hline C & 1.982580 & 2.136100 & -1.410890 & $\mathrm{C}$ & 2.964770 & 2.050950 & -2.141350 \\
\hline C & -0.888080 & 4.242860 & -1.138380 & $\mathrm{C}$ & 0.113580 & 4.197770 & -2.273650 \\
\hline C & -2.119020 & 3.547150 & -1.154710 & $\mathrm{C}$ & -1.125040 & 3.520320 & -2.258670 \\
\hline C & -3.419970 & 4.194000 & -1.148930 & $\mathrm{C}$ & -2.414820 & 4.184780 & -2.335430 \\
\hline C & -4.354750 & 3.192410 & -1.147070 & $\mathrm{C}$ & -3.365760 & 3.199220 & -2.301480 \\
\hline C & -3.621450 & 1.938550 & -1.154430 & $\mathrm{C}$ & -2.651000 & 1.936500 & -2.219960 \\
\hline $\mathrm{N}$ & -2.265120 & 2.177970 & -1.169580 & $\mathrm{~N}$ & -1.292250 & 2.155810 & -2.194580 \\
\hline $\mathrm{Ni}$ & -0.763080 & 0.781060 & -1.021920 & $\mathrm{Ni}$ & 0.179920 & 0.759460 & -1.858540 \\
\hline $\mathrm{N}$ & 0.711830 & -0.606590 & -1.371930 & $\mathrm{~N}$ & 1.660040 & -0.663350 & -1.970870 \\
\hline C & 0.561070 & -1.973470 & -1.411770 & $\mathrm{C}$ & 1.490050 & -2.020290 & -1.826850 \\
\hline C & 1.843040 & -2.617240 & -1.639490 & $\mathrm{C}$ & 2.779250 & -2.687050 & -1.748860 \\
\hline C & 2.773520 & -1.613770 & -1.728290 & $\mathrm{C}$ & 3.732080 & -1.706200 & -1.837050 \\
\hline C & 2.056310 & -0.364860 & -1.540700 & $\mathrm{C}$ & 3.018840 & -0.446880 & -1.966150 \\
\hline C & -4.231350 & 0.664930 & -1.127260 & $\mathrm{C}$ & -3.277150 & 0.670860 & -2.170080 \\
\hline C & -3.536530 & -0.564690 & -1.132420 & $\mathrm{C}$ & -2.600410 & -0.567730 & -2.107450 \\
\hline C & -4.183790 & -1.866310 & -1.112600 & $\mathrm{C}$ & -3.263660 & -1.859340 & -2.054930 \\
\hline C & -3.183320 & -2.801570 & -1.129700 & $\mathrm{C}$ & -2.276110 & -2.800810 & -1.922120 \\
\hline C & -1.931420 & -2.066330 & -1.185010 & $\mathrm{C}$ & -1.014990 & -2.080260 & -1.913920 \\
\hline $\mathrm{N}$ & -2.169330 & -0.710630 & -1.179020 & $\mathrm{~N}$ & -1.234720 & -0.727740 & -2.044530 \\
\hline C & -0.659720 & -2.671270 & -1.272760 & $\mathrm{C}$ & 0.249550 & -2.691960 & -1.772020 \\
\hline C & 2.668960 & 0.907480 & -1.530490 & $\mathrm{C}$ & 3.644160 & 0.816670 & -2.040510 \\
\hline $\mathrm{H}$ & -5.446450 & 3.296190 & -1.140560 & $\mathrm{H}$ & -4.455230 & 3.319450 & -2.338650 \\
\hline $\mathrm{H}$ & -3.597540 & 5.276310 & -1.163490 & $\mathrm{H}$ & -2.573150 & 5.267170 & -2.416130 \\
\hline $\mathrm{H}$ & -5.266160 & -2.042960 & -1.098240 & $\mathrm{H}$ & -4.346000 & -2.026340 & -2.120220 \\
\hline $\mathrm{H}$ & -3.285410 & -3.893550 & -1.122110 & $\mathrm{H}$ & -2.393300 & -3.888990 & -1.849080 \\
\hline $\mathrm{H}$ & 3.848140 & -1.708910 & -1.929010 & $\mathrm{H}$ & 4.822260 & -1.825280 & -1.816530 \\
\hline $\mathrm{H}$ & 2.003680 & -3.697000 & -1.749770 & $\mathrm{H}$ & 2.935530 & -3.769030 & -1.656270 \\
\hline $\mathrm{H}$ & 1.753060 & 5.454620 & -1.140560 & $\mathrm{H}$ & 2.761100 & 5.373180 & -2.379300 \\
\hline $\mathrm{H}$ & 3.718990 & 3.606630 & -1.379200 & $\mathrm{H}$ & 4.714980 & 3.496530 & -2.286250 \\
\hline C & -0.947790 & 5.727800 & -0.988670 & $\mathrm{C}$ & 0.081160 & 5.691450 & -2.328120 \\
\hline C & 4.157520 & 0.956190 & -1.648460 & $\mathrm{C}$ & 5.135160 & 0.863060 & -1.951270 \\
\hline $\mathrm{C}$ & -5.725000 & 0.617260 & -1.085980 & $\mathrm{C}$ & -4.770240 & 0.643560 & -2.123150 \\
\hline
\end{tabular}




\begin{tabular}{|c|c|c|c|c|c|c|c|}
\hline C & -0.578510 & -4.160410 & -1.179700 & C & 0.281670 & -4.151970 & -1.453020 \\
\hline C & -0.092040 & -4.759620 & -0.002210 & C & 0.540540 & -4.566720 & -0.133330 \\
\hline C & 0.034170 & -6.151140 & 0.130410 & C & 0.574120 & -5.920060 & 0.233540 \\
\hline C & -0.346460 & -6.978820 & -0.940350 & C & 0.349050 & -6.902170 & -0.745990 \\
\hline C & -0.842190 & -6.410210 & -2.126850 & C & 0.094140 & -6.520270 & -2.074670 \\
\hline C & -0.956440 & -5.013140 & -2.233250 & C & 0.056670 & -5.155550 & -2.412650 \\
\hline $\mathrm{F}$ & 0.267570 & -3.990460 & 1.033870 & $\mathrm{~F}$ & 0.752290 & -3.648910 & 0.823000 \\
\hline $\mathrm{F}$ & 0.501730 & -6.689010 & 1.260190 & $\mathrm{~F}$ & 0.806270 & -6.275300 & 1.502420 \\
\hline $\mathrm{F}$ & -0.236100 & -8.302940 & -0.831890 & $\mathrm{~F}$ & 0.377210 & -8.193450 & -0.416360 \\
\hline $\mathrm{F}$ & -1.197780 & -7.198310 & -3.144220 & $\mathrm{~F}$ & -0.110890 & -7.455240 & -3.004890 \\
\hline $\mathrm{F}$ & -1.428690 & -4.502410 & -3.374600 & $\mathrm{~F}$ & -0.201450 & -4.824850 & -3.681270 \\
\hline C & -6.411670 & 0.230060 & 0.081340 & C & -5.446210 & 0.325300 & -0.929670 \\
\hline C & -7.815090 & 0.188960 & 0.137260 & C & -6.847710 & 0.312870 & -0.846820 \\
\hline C & -8.562190 & 0.541560 & -0.999420 & C & -7.606540 & 0.628820 & -1.987080 \\
\hline C & -7.904670 & 0.931620 & -2.178530 & C & -6.961170 & 0.952860 & -3.192710 \\
\hline C & -6.500210 & 0.964760 & -2.209560 & C & -5.556670 & 0.953340 & -3.249230 \\
\hline $\mathrm{F}$ & -5.724790 & -0.106970 & 1.178620 & $\mathrm{~F}$ & -4.746110 & 0.031190 & 0.179690 \\
\hline $\mathrm{F}$ & -8.442950 & -0.175230 & 1.258520 & $\mathrm{~F}$ & -7.462730 & 0.013120 & 0.300920 \\
\hline $\mathrm{F}$ & -9.894390 & 0.506980 & -0.958920 & $\mathrm{~F}$ & -8.938120 & 0.623820 & -1.923300 \\
\hline $\mathrm{F}$ & -8.617230 & 1.263510 & -3.257590 & $\mathrm{~F}$ & -7.684770 & 1.254630 & -4.272580 \\
\hline $\mathrm{F}$ & -5.901910 & 1.340820 & -3.345060 & $\mathrm{~F}$ & -4.970320 & 1.264170 & -4.409290 \\
\hline C & 4.993490 & 0.535200 & -0.596610 & C & 5.765290 & 1.316270 & -0.776050 \\
\hline C & 6.393530 & 0.581650 & -0.692420 & C & 7.163120 & 1.391390 & -0.663850 \\
\hline C & 6.988880 & 1.066860 & -1.869740 & C & 7.962720 & 1.000850 & -1.751980 \\
\hline C & 6.182090 & 1.493070 & -2.938700 & C & 7.362140 & 0.538760 & -2.935680 \\
\hline C & 4.783280 & 1.434090 & -2.816990 & C & 5.961020 & 0.470480 & -3.021440 \\
\hline $\mathrm{F}$ & 4.458710 & 0.073390 & 0.545810 & $\mathrm{~F}$ & 5.026510 & 1.688800 & 0.277550 \\
\hline $\mathrm{F}$ & 7.158490 & 0.180530 & 0.326980 & $\mathrm{~F}$ & 7.733890 & 1.825190 & 0.462500 \\
\hline $\mathrm{F}$ & 8.316800 & 1.119290 & -1.972720 & $\mathrm{~F}$ & 9.291200 & 1.068300 & -1.660900 \\
\hline $\mathrm{F}$ & 6.746450 & 1.947870 & -4.059340 & $\mathrm{~F}$ & 8.123750 & 0.172970 & -3.969500 \\
\hline $\mathrm{F}$ & 4.043450 & 1.840390 & -3.852370 & $\mathrm{~F}$ & 5.418840 & 0.024590 & -4.159190 \\
\hline C & -0.595950 & 6.600090 & -2.037400 & C & 0.423440 & 6.392950 & -3.500190 \\
\hline C & -0.671780 & 7.996320 & -1.896680 & C & 0.400300 & 7.797090 & -3.555970 \\
\hline C & -1.109000 & 8.548590 & -0.680470 & C & 0.025900 & 8.527940 & -2.415720 \\
\hline C & -1.463540 & 7.703920 & 0.385810 & C & -0.322050 & 7.854550 & -1.232480 \\
\hline C & -1.374280 & 6.313100 & 0.219550 & C & -0.292640 & 6.450200 & -1.202110 \\
\hline $\mathrm{F}$ & -0.181740 & 6.108090 & -3.208580 & $\mathrm{~F}$ & 0.782670 & 5.720980 & -4.598920 \\
\hline $\mathrm{F}$ & -0.338200 & 8.797720 & -2.910240 & $\mathrm{~F}$ & 0.725890 & 8.441100 & -4.679740 \\
\hline $\mathrm{F}$ & -1.186590 & 9.871370 & -0.537230 & $\mathrm{~F}$ & 0.000552 & 9.860550 & -2.456320 \\
\hline $\mathrm{F}$ & -1.874870 & 8.225640 & 1.544320 & $\mathrm{~F}$ & -0.672720 & 8.552960 & -0.149510 \\
\hline $\mathrm{F}$ & -1.708410 & 5.534700 & 1.263570 & $\mathrm{~F}$ & -0.629090 & 5.834200 & -0.063060 \\
\hline C & -0.622960 & 2.602510 & 4.167500 & C & -1.680660 & 0.440370 & 3.297220 \\
\hline $\mathrm{H}$ & -1.068600 & 2.074790 & 5.034530 & $\mathrm{H}$ & -1.716760 & -0.589030 & 3.712260 \\
\hline $\mathrm{H}$ & 0.378140 & 2.981260 & 4.466140 & $\mathrm{H}$ & -1.430220 & 1.146490 & 4.114810 \\
\hline H & -1.260880 & 3.453710 & 3.855690 & $\mathrm{H}$ & -2.673360 & 0.708230 & 2.881020 \\
\hline 0 & 3.758460 & -4.853580 & 1.975370 & 0 & -0.679170 & -4.764830 & 5.502190 \\
\hline C & 4.752570 & -4.447620 & 1.050220 & C & -1.166320 & -5.153030 & 6.775450 \\
\hline H & 5.551100 & -3.843030 & 1.541740 & $\mathrm{H}$ & -2.055160 & -4.550020 & 7.077460 \\
\hline $\mathrm{H}$ & 5.198460 & -5.380420 & 0.649960 & $\mathrm{H}$ & -1.464250 & -6.216160 & 6.679060 \\
\hline $\mathrm{H}$ & 4.314370 & -3.857110 & 0.210630 & $\mathrm{H}$ & -0.380140 & -5.063070 & 7.561510 \\
\hline $\mathrm{H}$ & 3.971330 & -2.112710 & 1.756150 & $\mathrm{H}$ & 0.280020 & -3.989020 & 3.275660 \\
\hline $\mathrm{H}$ & 1.959960 & -5.459500 & 3.673330 & $\mathrm{H}$ & -0.575920 & -2.716520 & 7.348570 \\
\hline
\end{tabular}




\section{trans- $\alpha$}

$E_{P B E / S V P}=-6117.428319313$ Hartree

NImag $=0$

\begin{tabular}{|c|c|c|c|}
\hline C & -2.701870 & -0.188400 & 4.253120 \\
\hline C & -4.091290 & -0.356390 & 4.014160 \\
\hline C & -4.988790 & -0.440290 & 5.074300 \\
\hline C & -4.522240 & -0.356430 & 6.408630 \\
\hline C & -3.136260 & -0.187170 & 6.658800 \\
\hline C & -2.242080 & -0.104390 & 5.590250 \\
\hline$N$ & -1.894520 & -0.124860 & 3.115420 \\
\hline$N$ & -0.638850 & 0.023530 & 3.312750 \\
\hline $\mathrm{C}$ & 0.089050 & 0.071080 & 2.145790 \\
\hline C & -0.234760 & -0.006180 & 0.787210 \\
\hline $\mathrm{N}$ & 1.475680 & 0.227050 & 2.194610 \\
\hline$N$ & 0.908440 & 0.098320 & 0.049230 \\
\hline $\mathrm{C}$ & 1.923620 & 0.238260 & 0.912210 \\
\hline $\mathrm{H}$ & -4.431950 & -0.417390 & 2.967 \\
\hline $\mathrm{H}$ & -1.163070 & 0.025510 & 5.765190 \\
\hline $\mathrm{H}$ & -1.229030 & -0.131000 & 0.346050 \\
\hline $\mathrm{H}$ & 2.982160 & 0.348540 & 0.639910 \\
\hline $\mathrm{C}$ & 2.268090 & 2.856940 & -2.258630 \\
\hline$N$ & 2.449810 & 1.494300 & -2.190130 \\
\hline $\mathrm{C}$ & 3.550770 & 3.538980 & -2.237250 \\
\hline C & 4.511520 & 2.564990 & -2.150440 \\
\hline $\mathrm{C}$ & 3.809680 & 1.293420 & -2.121370 \\
\hline C & 1.020550 & 3.515740 & -2.326660 \\
\hline C & -0.236060 & 2.872400 & -2.329060 \\
\hline C & -1.507910 & 3.573430 & -2.383400 \\
\hline C & -2.485500 & 2.615880 & -2.325320 \\
\hline $\mathrm{C}$ & -1.805400 & 1.332920 & -2.263900 \\
\hline$N$ & -0.441040 & 1.512620 & -2.272410 \\
\hline $\mathrm{Ni}$ & 0.989860 & 0.062790 & -1.979330 \\
\hline$N$ & 2.428720 & -1.395410 & -2.149120 \\
\hline C & 2.225930 & -2.756150 & -2.181400 \\
\hline $\mathrm{C}$ & 3.499240 & -3.456320 & -2.171200 \\
\hline C & 4.474420 & -2.496170 & -2.105570 \\
\hline C & 3.792020 & -1.213370 & -2.098900 \\
\hline C & -2.465520 & 0.085220 & -2.205870 \\
\hline C & -1.822700 & -1.172350 & -2.218090 \\
\hline C & -2.524120 & -2.444440 & -2.237690 \\
\hline C & -1.562400 & -3.420180 & -2.251020 \\
\hline $\mathrm{C}$ & -0.278750 & -2.738790 & -2.237350 \\
\hline$N$ & -0.461320 & -1.374520 & -2.225210 \\
\hline C & 0.969130 & -3.399840 & -2.218710 \\
\hline $\mathrm{C}$ & 4.449420 & 0.035980 & -2.056030 \\
\hline $\mathrm{H}$ & -3.571660 & 2.766680 & -2.335060 \\
\hline $\mathrm{H}$ & -1.637550 & 4.659970 & -2.458850 \\
\hline $\mathrm{H}$ & -3.613300 & -2.574170 & -2.254880 \\
\hline $\mathrm{H}$ & -1.710660 & -4.506810 & -2.265820 \\
\hline $\mathrm{H}$ & 5.560640 & -2.645570 & -2.076890 \\
\hline $\mathrm{H}$ & 3.632320 & -4.544090 & -2.216980 \\
\hline $\mathrm{H}$ & 3.698880 & 4.624850 & -2.28378 \\
\hline $\mathrm{H}$ & 5.600620 & 2.695200 & -2.126230 \\
\hline $\mathrm{C}$ & 1.029720 & 5.009940 & -2.37902 \\
\hline $\mathrm{C}$ & 5.937970 & 0.025440 & -1.92452 \\
\hline $\mathrm{C}$ & -3.955410 & 0.098770 & -2.086430 \\
\hline C & 0.961250 & -4.894460 & -2.2211 \\
\hline $\mathrm{C}$ & 1.318390 & -5.627440 & -1.0724 \\
\hline
\end{tabular}

trans- $\beta$

$E_{P B E / S V P}=-6117.426950607$ Hartree NImag $=0$

\begin{tabular}{|c|c|c|c|}
\hline C & -1.114090 & 0.006710 & 5.329630 \\
\hline C & -0.477720 & 0.064040 & 6.588580 \\
\hline c & -1.213780 & 0.023470 & 7.778660 \\
\hline C & -2.622140 & -0.075580 & 7.720540 \\
\hline C & -3.270060 & -0.133980 & 6.458000 \\
\hline C & -2.531400 & -0.093870 & 5.282880 \\
\hline$N$ & -0.277980 & 0.055880 & 4.204550 \\
\hline$N$ & -0.881500 & -0.004660 & 3.077400 \\
\hline C & -0.099600 & 0.042710 & 1.955040 \\
\hline$C$ & -0.574970 & -0.016720 & 0.638380 \\
\hline $\mathrm{N}$ & 1.300490 & 0.157190 & 1.839370 \\
\hline $\mathrm{N}$ & 0.473660 & 0.056870 & -0.231340 \\
\hline$C$ & 1.580570 & 0.160440 & 0.512380 \\
\hline $\mathrm{H}$ & 0.621110 & 0.141240 & 6.611330 \\
\hline $\mathrm{H}$ & -3.020050 & -0.138000 & 4.297840 \\
\hline $\mathrm{H}$ & -1.618370 & -0.111990 & 0.314440 \\
\hline $\mathrm{H}$ & 2.603030 & 0.244430 & 0.118410 \\
\hline$C$ & 2.306600 & 0.256940 & 2.890150 \\
\hline$C$ & 1.745780 & 2.807290 & -2.570360 \\
\hline $\mathrm{N}$ & 1.929180 & 1.444180 & -2.506130 \\
\hline$C$ & 3.028210 & 3.489440 & -2.579000 \\
\hline$C$ & 3.990640 & 2.516170 & -2.509800 \\
\hline$C$ & 3.290370 & 1.244260 & -2.459020 \\
\hline$C$ & 0.497640 & 3.466980 & -2.604940 \\
\hline$C$ & -0.758870 & 2.823360 & -2.590750 \\
\hline$C$ & -2.031400 & 3.523360 & -2.639660 \\
\hline$C$ & -3.007920 & 2.563780 & -2.598800 \\
\hline$C$ & -2.326310 & 1.281440 & -2.544780 \\
\hline $\mathrm{N}$ & -0.962270 & 1.463190 & -2.540770 \\
\hline $\mathrm{Ni}$ & 0.467820 & 0.013680 & -2.264610 \\
\hline $\mathrm{N}$ & 1.913210 & -1.444470 & -2.445300 \\
\hline$C$ & 1.710440 & -2.805740 & -2.458750 \\
\hline$C$ & 2.983580 & -3.505970 & -2.439490 \\
\hline$C$ & 3.959010 & -2.545320 & -2.385850 \\
\hline$C$ & 3.277070 & -1.262470 & -2.399540 \\
\hline$C$ & -2.984960 & 0.032690 & -2.512600 \\
\hline$C$ & -2.340070 & -1.223790 & -2.509900 \\
\hline$C$ & -3.039480 & -2.496870 & -2.542130 \\
\hline$C$ & -2.076950 & -3.471620 & -2.537550 \\
\hline$C$ & -0.794380 & -2.789110 & -2.507380 \\
\hline $\mathrm{N}$ & -0.978620 & -1.424970 & -2.493850 \\
\hline$C$ & 0.453790 & -3.449620 & -2.488670 \\
\hline$C$ & 3.933610 & -0.011880 & -2.377090 \\
\hline $\mathrm{H}$ & -4.094400 & 2.712540 & -2.612710 \\
\hline $\mathrm{H}$ & -2.162070 & 4.610400 & -2.705290 \\
\hline $\mathrm{H}$ & -4.127890 & -2.628290 & -2.578000 \\
\hline $\mathrm{H}$ & -2.224160 & -4.558310 & -2.554040 \\
\hline $\mathrm{H}$ & 5.045120 & -2.694850 & -2.355320 \\
\hline $\mathrm{H}$ & 3.116380 & -4.594280 & -2.470270 \\
\hline $\mathrm{H}$ & 3.175610 & 4.575010 & -2.632190 \\
\hline $\mathrm{H}$ & 5.079670 & 2.648190 & -2.511420 \\
\hline C & 0.505150 & 4.961380 & -2.645680 \\
\hline C & 5.420540 & -0.005590 & -2.228250 \\
\hline C & -4.478420 & 0.038320 & -2.467410 \\
\hline C & 0.445350 & -4.944400 & -2.492850 \\
\hline
\end{tabular}




\begin{tabular}{|c|c|c|c|c|c|c|c|}
\hline$c$ & 1.318080 & -7.032330 & -1.059740 & C & 0.795870 & -5.680080 & -1.344120 \\
\hline E & 0.957410 & -7.733970 & -2.222460 & C & 0.795160 & -7.085070 & -1.334970 \\
\hline c & 0.595500 & -7.030050 & -3.383400 & C & 0.433500 & -7.783710 & -2.499210 \\
\hline C & 0.602930 & -5.624670 & -3.371000 & C & 0.079250 & -7.076880 & -3.660770 \\
\hline $\mathrm{F}$ & 1.665650 & -4.985130 & 0.049090 & C & 0.087980 & -5.671610 & -3.645010 \\
\hline $\mathrm{F}$ & 1.656270 & -7.705200 & 0.043740 & $\mathrm{~F}$ & 1.141680 & -5.040280 & -0.220660 \\
\hline $\mathrm{F}$ & 0.955370 & -9.067570 & -2.222850 & $\mathrm{~F}$ & 1.128280 & -7.760690 & -0.231780 \\
\hline $\mathrm{F}$ & 0.256510 & -7.701030 & -4.487110 & $\mathrm{~F}$ & 0.428460 & -9.117220 & -2.502130 \\
\hline $\mathrm{F}$ & 0.257710 & -4.980030 & -4.490370 & $\mathrm{~F}$ & -0.258490 & -7.744950 & -4.766570 \\
\hline C & -4.577100 & -0.203280 & -0.859240 & $\mathrm{~F}$ & -0.252210 & -5.023940 & -4.764180 \\
\hline C & -5.970760 & -0.156390 & -0.698170 & C & -5.163030 & -0.298760 & -1.284020 \\
\hline C & -6.780230 & 0.182130 & -1.795940 & C & -6.565890 & -0.300070 & -1.212380 \\
\hline C & -6.189540 & 0.479980 & -3.036350 & C & -7.313690 & 0.048840 & -2.350100 \\
\hline C & -4.791010 & 0.433140 & -3.168670 & C & -6.658000 & 0.389330 & -3.545540 \\
\hline $\mathrm{F}$ & -3.833070 & -0.526200 & 0.208290 & C & -5.253400 & 0.382280 & -3.591360 \\
\hline $\mathrm{F}$ & -6.527080 & -0.425030 & 0.488350 & $\mathrm{~F}$ & -4.472100 & -0.627400 & -0.183560 \\
\hline $\mathrm{F}$ & -8.105960 & 0.227620 & -1.659900 & $\mathrm{~F}$ & -7.192880 & -0.618950 & -0.076820 \\
\hline $\mathrm{F}$ & -6.958530 & 0.800230 & -4.079600 & $\mathrm{~F}$ & -8.645990 & 0.054320 & -2.295680 \\
\hline $\mathrm{F}$ & -4.259200 & 0.723880 & -4.360320 & $\mathrm{~F}$ & -7.372630 & 0.716380 & -4.624650 \\
\hline C & 6.555880 & 0.331970 & -0.697850 & $\mathrm{~F}$ & -4.655310 & 0.710780 & -4.741190 \\
\hline C & 7.951000 & 0.306750 & -0.540360 & C & 6.017330 & 0.392410 & -1.016630 \\
\hline C & 8.761480 & -0.024260 & -1.640170 & C & 7.410120 & 0.437250 & -0.848620 \\
\hline C & 8.173510 & -0.332620 & -2.878950 & C & 8.243490 & 0.064210 & -1.917520 \\
\hline C & 6.774460 & -0.301840 & -3.008430 & C & 7.678620 & -0.345610 & -3.137520 \\
\hline $\mathrm{F}$ & 5.805450 & 0.647580 & 0.371030 & C & 6.280560 & -0.378470 & -3.278890 \\
\hline $\mathrm{F}$ & 8.510870 & 0.589230 & 0.639570 & $\mathrm{~F}$ & 5.246780 & 0.745070 & 0.027920 \\
\hline $\mathrm{F}$ & 10.087740 & -0.051370 & -1.505760 & $\mathrm{~F}$ & 7.944620 & 0.823480 & 0.313170 \\
\hline $\mathrm{F}$ & 8.944650 & -0.646870 & -3.921930 & $\mathrm{~F}$ & 9.568320 & 0.099700 & -1.773500 \\
\hline $\mathrm{F}$ & 6.242460 & -0.601240 & -4.197210 & $\mathrm{~F}$ & 8.470320 & -0.696500 & -4.153020 \\
\hline C & 1.404980 & 5.699160 & -3.548410 & $\mathrm{~F}$ & 5.775150 & -0.772780 & -4.451270 \\
\hline C & 1.414420 & 7.103310 & -3.609890 & C & 0.871030 & 5.661880 & -3.811350 \\
\hline C & 1.041540 & 7.846980 & -2.477500 & C & 0.876870 & 7.066520 & -3.860150 \\
\hline C & 0.664020 & 7.186110 & -1.296250 & C & 0.512140 & 7.799240 & -2.718000 \\
\hline C & 0.660080 & 5.781610 & -1.260580 & C & 0.142970 & 7.127100 & -1.540500 \\
\hline $\mathrm{F}$ & 1.762750 & 5.015070 & -4.639670 & C & 0.143480 & 5.722310 & -1.517130 \\
\hline $\mathrm{F}$ & 1.768430 & 7.735220 & -4.732050 & $\mathrm{~F}$ & 1.222290 & 4.988360 & -4.911480 \\
\hline $\mathrm{F}$ & 1.046970 & 9.179840 & -2.523060 & $\mathrm{~F}$ & 1.221930 & 7.709430 & -4.978740 \\
\hline $\mathrm{F}$ & 0.316110 & 7.896280 & -0.219710 & $\mathrm{~F}$ & 0.515160 & 9.132370 & -2.751570 \\
\hline $\mathrm{F}$ & 0.296220 & 5.179000 & -0.122440 & $\mathrm{~F}$ & -0.198590 & 7.827100 & -0.455460 \\
\hline C & 2.277740 & 0.353450 & 3.401020 & $\mathrm{~F}$ & -0.210510 & 5.108190 & -0.382170 \\
\hline $\mathrm{H}$ & 1.942830 & 1.229090 & 3.994800 & $\mathrm{H}$ & 3.301140 & 0.336360 & 2.403050 \\
\hline $\mathrm{H}$ & 2.177170 & -0.556460 & 4.028340 & $\mathrm{H}$ & 2.272690 & -0.636350 & 3.544200 \\
\hline $\mathrm{H}$ & 3.338580 & 0.487150 & 3.108920 & $\mathrm{H}$ & 2.119850 & 1.147370 & 3.521710 \\
\hline 0 & -5.468100 & -0.450610 & 7.374120 & 0 & -3.437120 & -0.122170 & 8.802110 \\
\hline C & -5.082420 & -0.354580 & 8.734570 & C & -2.866840 & -0.057360 & 10.097850 \\
\hline $\mathrm{H}$ & -4.586750 & 0.620110 & 8.954910 & $\mathrm{H}$ & -2.305450 & 0.894030 & 10.253040 \\
\hline $\mathrm{H}$ & -6.018050 & -0.427470 & 9.324440 & $\mathrm{H}$ & -3.715760 & -0.098940 & 10.809590 \\
\hline $\mathrm{H}$ & -4.399910 & -1.186670 & 9.028490 & $\mathrm{H}$ & -2.185160 & -0.918950 & 10.290760 \\
\hline $\mathrm{H}$ & -6.069080 & -0.571720 & 4.907330 & $\mathrm{H}$ & -0.686790 & 0.068510 & 8.742670 \\
\hline $\mathrm{H}$ & -2.754490 & -0.122160 & 7.688630 & $\mathrm{H}$ & -4.368900 & -0.211010 & 6.444670 \\
\hline
\end{tabular}


I.6.7 1-Methyl-3',5'-dimethyl-4'-methoxy-5-phenylazoimidazole - NiTPPF 20 (2f)

\section{cis- $\alpha$}

$E_{P B E} / \mathrm{SVP}=-6195.859320536$ Hartree

NImag $=0$

\begin{tabular}{|c|c|c|c|}
\hline C & 1.342090 & -1.982400 & 4.012460 \\
\hline C & 1.089260 & -3.363590 & 4.153090 \\
\hline C & 1.837470 & -4.314520 & 3.443240 \\
\hline C & 2.905770 & -3.855780 & 2.632950 \\
\hline C & 3.216520 & -2.475920 & 2.514840 \\
\hline C & 2.413540 & -1.550300 & 3.199180 \\
\hline$N$ & 0.599970 & -1.106640 & 4.842040 \\
\hline $\mathrm{N}$ & 0.098880 & -0.012250 & 4.457610 \\
\hline C & -0.036200 & 0.424190 & 3.144110 \\
\hline C & -0.089780 & -0.110070 & 1.842310 \\
\hline$N$ & -0.472330 & 1.750800 & 3.006290 \\
\hline$N$ & -0.531950 & 0.851010 & 0.979370 \\
\hline $\mathrm{C}$ & -0.741060 & 1.962070 & 1.700730 \\
\hline $\mathrm{H}$ & 0.273970 & -3.681170 & 4.823070 \\
\hline $\mathrm{H}$ & 2.638230 & -0.475250 & 3.109860 \\
\hline $\mathrm{H}$ & 0.152560 & -1.123900 & 1.504500 \\
\hline $\mathrm{H}$ & -1.069860 & 2.929690 & 1.299250 \\
\hline C & 0.355300 & 3.682800 & -1.282300 \\
\hline$N$ & 0.604160 & 2.331660 & -1.366310 \\
\hline C & 1.600250 & 4.430350 & -1.338320 \\
\hline C & 2.604510 & 3.507990 & -1.470380 \\
\hline C & 1.969820 & 2.200920 & -1.476120 \\
\hline C & -0.923580 & 4.272640 & -1.158660 \\
\hline C & -2.144750 & 3.561010 & -1.112970 \\
\hline C & -3.451630 & 4.189470 & -1.023960 \\
\hline C & -4.370210 & 3.174940 & -0.959360 \\
\hline C & -3.622480 & 1.931550 & -1.022380 \\
\hline$N$ & -2.272950 & 2.189650 & -1.122900 \\
\hline $\mathrm{Ni}$ & -0.746570 & 0.805880 & -1.050650 \\
\hline$N$ & 0.733120 & -0.556590 & -1.478540 \\
\hline C & 0.597940 & -1.923690 & -1.565820 \\
\hline C & 1.891690 & -2.549390 & -1.778130 \\
\hline C & 2.813780 & -1.536070 & -1.810170 \\
\hline C & 2.077300 & -0.297870 & -1.623230 \\
\hline C & -4.212220 & 0.648710 & -0.985250 \\
\hline C & -3.504290 & -0.569880 & -1.085520 \\
\hline C & -4.139100 & -1.876910 & -1.130930 \\
\hline C & -3.133380 & -2.796040 & -1.272120 \\
\hline C & -1.890750 & -2.045110 & -1.317530 \\
\hline$N$ & -2.139180 & -0.696330 & -1.200500 \\
\hline C & -0.618830 & -2.633520 & -1.481030 \\
\hline $\mathrm{C}$ & 2.671610 & 0.981950 & -1.601300 \\
\hline $\mathrm{H}$ & -5.460400 & 3.263720 & -0.880730 \\
\hline $\mathrm{H}$ & -3.644890 & 5.269180 & -1.025910 \\
\hline $\mathrm{H}$ & -5.218250 & -2.066210 & -1.081360 \\
\hline $\mathrm{H}$ & -3.224220 & -3.886530 & -1.349160 \\
\hline $\mathrm{H}$ & 3.897730 & -1.619910 & -1.955150 \\
\hline $\mathrm{H}$ & 2.070830 & -3.625520 & -1.892600 \\
\hline $\mathrm{H}$ & 1.697230 & 5.521650 & -1.291860 \\
\hline $\mathrm{H}$ & 3.680710 & 3.697980 & -1.563960 \\
\hline C & -0.991190 & 5.758920 & -1.023180 \\
\hline C & 4.159370 & 1.057980 & -1.727440 \\
\hline C & -5.698090 & 0.568310 & -0.843220 \\
\hline
\end{tabular}

cis- $\beta$

$E_{P B E / S V P}=-6195.848580914$ Hartree NImag $=0$

C $\quad 0.409850 \quad-0.762970$

C $\quad-0.190550-0.894170$

C $\quad-0.888190-2.054610$

C $\quad-0.916820 \quad-3.133390$

C $\quad-0.294590 \quad-3.046020$

C $\quad 0.353270 \quad-1.847750$

N $\quad 1.179680 \quad 0.404640$

$\begin{array}{lll}\mathrm{N} & 1.354120 & 0.979090\end{array}$

C $\quad 0.619550 \quad 0.785080$

C $\quad 1.135990 \quad 0.911260$

N $\quad-0.767600 \quad 0.611780$

N $\quad 0.127300 \quad 0.768160$

C $\quad-1.001680 \quad 0.619840$

H $\quad-0.103200 \quad-0.049670$

$\mathrm{H} \quad 0.844510 \quad-1.772560$

H $\quad 2.183530 \quad 1.067460$

H $\quad-2.011250 \quad 0.556590$

C $\quad-1.816040 \quad 0.601480$

C $\quad 1.422760 \quad 3.565520$

$\begin{array}{lll}\mathrm{N} & 1.647380 & 2.215570\end{array}$

$\begin{array}{lll}\mathrm{N} & 1.647380 & 2.215570 \\ \mathrm{C} & 2.681230 & 4.291580\end{array}$

C $\quad 3.670350 \quad 3.357890$

$\begin{array}{lll}\text { C } & 3.011270 \quad 2.065040\end{array}$

C $\quad 0.158660 \quad 4.178250$

C $\quad-1.077350 \quad 3.494540$

C $\quad-2.371230 \quad 4.143120$

C $\quad-3.316860 \quad 3.158600$

$\begin{array}{lll}\text { C } & -3.316860 & 3.158600 \\ \text { C } & -2.594710 & 1.907990\end{array}$

$\mathrm{N} \quad-1.236950 \quad 2.135500$

$\mathrm{Ni} \quad 0.238270 \quad 0.743210$

N $\quad 1.728890 \quad-0.667530$

C $\quad 1.573410 \quad-2.034810$

C $\quad 2.870230 \quad-2.690000$

C $\quad 3.809650 \quad-1.695740$

$\begin{array}{lll}\text { C } & 3.809650 & -1.695740 \\ \text { C } & 3.084040 & -0.437570\end{array}$

$\begin{array}{lll}\text { C } & -3.212300 & 0.641570\end{array}$

C $\quad-2.529160 \quad-0.593990$

C $\quad-3.187000-1.888540$

C $\quad-2.191680 \quad-2.831170$

C $\quad-0.932020 \quad-2.106780$

N $\quad-1.161490$

$-0.750230$

0.339660

-0.750230
-2.722020

$3.696520 \quad 0.834600$

$-4.407040$

3.270170

$-2.538340$

5.215190

$-4.271360$

$-2.055430$

$-2.304280$

$-3.922050$

4.899220

$-1.806380$

3.042830

$-3.772870$

2.794870

5.378330

3.528450

5.655930

0.895460
4.973620

6.247170

6.608030

5.685670

4.412480

4.065220

4.765580

3.651610

2.486620

1.191410

2.316820

0.279960

0.975990

6.949990

3.081440

0.906970

0.547260

3.328210

$-2.135510$

$-1.987950$

$-2.117940$

$-1.959540$

$-1.884530$

$-2.285750$

$-2.263010$

$-2.391110$

$-2.277680$

$-2.118930$

$-2.111460$

$-1.753490$

$-1.876590$

$-1.886460$

$-1.840740$

$-1.770660$

$-1.801600$

$-2.015100$

$-1.981720$

$-1.956700$

$-1.944660$

$-1.958170$

$-1.986830$

$-1.921520$

$-1.770850$

$-2.320060$

$-2.550520$

$-1.965620$

$-1.927040$

$-1.714230$

$-1.866290$

$-2.211570$

$-1.916760$

$-2.508440$

$-1.611610$ 


\begin{tabular}{|c|c|c|c|c|c|c|c|}
\hline C & -0.551530 & -4.123220 & -1.595090 & C & -4.703290 & 0.611550 & -1.910310 \\
\hline C & -0.187220 & -4.921850 & -0.495560 & C & 0.379260 & -4.214760 & -1.872170 \\
\hline E & -0.111360 & -6.320810 & -0.595910 & C & 0.770110 & -4.889860 & -0.700280 \\
\hline$C$ & -0.395840 & -6.945550 & -1.822040 & C & 0.794350 & -6.290740 & -0.615420 \\
\hline C & -0.762210 & -6.171670 & -2.935990 & C & 0.433740 & -7.053310 & -1.739480 \\
\hline C & -0.838490 & -4.774040 & -2.809800 & C & 0.043510 & -6.409990 & -2.926670 \\
\hline $\mathrm{F}$ & 0.094670 & -4.348440 & 0.682200 & C & 0.020450 & -5.005500 & -2.981280 \\
\hline $\mathrm{F}$ & 0.230080 & -7.068940 & 0.459620 & $\mathrm{~F}$ & 1.118180 & -4.186860 & 0.388920 \\
\hline $\mathrm{F}$ & -0.320430 & -8.272420 & -1.926120 & $\mathrm{~F}$ & 1.153530 & -6.899410 & 0.519220 \\
\hline $\mathrm{F}$ & -1.029150 & -6.766830 & -4.100960 & $\mathrm{~F}$ & 0.456680 & -8.384920 & -1.678330 \\
\hline $\mathrm{F}$ & -1.189820 & -4.059130 & -3.882200 & $\mathrm{~F}$ & -0.295760 & -7.136280 & -3.993690 \\
\hline C & -6.289700 & 0.101700 & 0.347180 & $\mathrm{~F}$ & -0.358200 & -4.424980 & -4.124100 \\
\hline C & -7.682980 & 0.000272 & 0.493670 & C & -5.329450 & 0.381750 & -0.671080 \\
\hline C & -8.519620 & 0.376660 & -0.570810 & C & -6.725940 & 0.386920 & -0.528840 \\
\hline C & -7.958670 & 0.848990 & -1.769680 & C & -7.531230 & 0.622820 & -1.656800 \\
\hline C & -6.561970 & 0.939470 & -1.892160 & C & -6.936260 & 0.851570 & -2.909310 \\
\hline $\mathrm{F}$ & -5.520830 & -0.260370 & 1.380140 & C & -5.535200 & 0.838660 & -3.023050 \\
\hline $\mathrm{F}$ & -8.214030 & -0.446100 & 1.634850 & $\mathrm{~F}$ & -4.584750 & 0.161090 & 0.428120 \\
\hline $\mathrm{F}$ & -9.843670 & 0.285690 & -0.443600 & $\mathrm{~F}$ & -7.291470 & 0.178510 & 0.663090 \\
\hline $\mathrm{F}$ & -8.752270 & 1.201850 & -2.783870 & $\mathrm{~F}$ & -8.858720 & 0.633070 & -1.536470 \\
\hline $\mathrm{F}$ & -6.060430 & 1.389100 & -3.047210 & $\mathrm{~F}$ & -7.702680 & 1.079260 & -3.977900 \\
\hline C & 4.970350 & 1.354250 & -0.616550 & $\mathrm{~F}$ & -4.996140 & 1.059810 & -4.225350 \\
\hline C & 6.366860 & 1.455450 & -0.718580 & C & 5.754840 & 1.349930 & -0.408260 \\
\hline C & 6.982150 & 1.256420 & -1.966290 & C & 7.145480 & 1.447180 & -0.238460 \\
\hline C & 6.200060 & 0.950560 & -3.093070 & C & 7.996560 & 1.077120 & -1.294190 \\
\hline C & 4.804050 & 0.855000 & -2.962500 & C & 7.453330 & 0.614380 & -2.504990 \\
\hline $\mathrm{F}$ & 4.411870 & 1.535070 & 0.590370 & C & 6.058430 & 0.528680 & -2.650030 \\
\hline $\mathrm{F}$ & 7.109260 & 1.738800 & 0.354800 & $\mathrm{~F}$ & 4.968780 & 1.704600 & 0.617190 \\
\hline $\mathrm{F}$ & 8.307040 & 1.352610 & -2.079600 & $\mathrm{~F}$ & 7.661030 & 1.883700 & 0.912790 \\
\hline $\mathrm{F}$ & 6.785400 & 0.761590 & -4.277780 & $\mathrm{~F}$ & 9.318720 & 1.164160 & -1.146480 \\
\hline $\mathrm{F}$ & 4.086400 & 0.561480 & -4.050820 & $\mathrm{~F}$ & 8.261970 & 0.266670 & -3.509040 \\
\hline C & -0.680740 & 6.620220 & -2.093900 & $\mathrm{~F}$ & 5.571840 & 0.089790 & -3.815440 \\
\hline C & -0.739540 & 8.018050 & -1.961290 & C & 0.609480 & 6.208080 & -3.714590 \\
\hline C & -1.122360 & 8.583260 & -0.732770 & C & 0.596810 & 7.592030 & -3.953700 \\
\hline C & -1.438270 & 7.749960 & 0.354460 & C & 0.092250 & 8.459080 & -2.969850 \\
\hline C & -1.365500 & 6.357100 & 0.196080 & C & -0.391900 & 7.937990 & -1.758000 \\
\hline $\mathrm{F}$ & -0.316580 & 6.115700 & -3.276680 & C & -0.363820 & 6.550090 & -1.540230 \\
\hline $\mathrm{F}$ & -0.439330 & 8.809080 & -2.993220 & $\mathrm{~F}$ & 1.089350 & 5.407980 & -4.672300 \\
\hline $\mathrm{F}$ & -1.183900 & 9.907730 & -0.597240 & $\mathrm{~F}$ & 1.053510 & 8.086140 & -5.106700 \\
\hline $\mathrm{F}$ & -1.796440 & 8.285160 & 1.524740 & $\mathrm{~F}$ & 0.072050 & 9.774710 & -3.185750 \\
\hline $\mathrm{F}$ & -1.661560 & 5.588960 & 1.259760 & $\mathrm{~F}$ & -0.869140 & 8.764480 & -0.823460 \\
\hline C & -0.593900 & 2.717240 & 4.087610 & $\mathrm{~F}$ & -0.826670 & 6.086090 & -0.373750 \\
\hline $\mathrm{H}$ & -1.165260 & 2.264060 & 4.922560 & $\mathrm{H}$ & -2.775410 & 0.869250 & 2.840260 \\
\hline $\mathrm{H}$ & 0.408470 & 3.006540 & 4.467890 & $\mathrm{H}$ & -1.587760 & 1.350160 & 4.113730 \\
\hline $\mathrm{H}$ & -1.114910 & 3.615650 & 3.700180 & $\mathrm{H}$ & -1.906210 & -0.398020 & 3.802270 \\
\hline C & 4.363880 & -2.019050 & 1.646860 & C & -0.359400 & -4.205780 & 3.448760 \\
\hline $\mathrm{H}$ & 5.350400 & -2.220940 & 2.124090 & $\mathrm{H}$ & -1.350870 & -4.704560 & 3.499630 \\
\hline $\mathrm{H}$ & 4.356210 & -2.555300 & 0.673950 & $\mathrm{H}$ & -0.171340 & -3.875490 & 2.407340 \\
\hline $\mathrm{H}$ & 4.306510 & -0.927610 & 1.453360 & $\mathrm{H}$ & 0.407010 & -4.980940 & 3.679970 \\
\hline C & 1.513770 & -5.785990 & 3.516280 & C & -1.587910 & -2.182230 & 7.939070 \\
\hline $\mathrm{H}$ & 0.663120 & -5.970180 & 4.205090 & $\mathrm{H}$ & -2.648100 & -2.489360 & 7.805810 \\
\hline $\mathrm{H}$ & 1.240860 & -6.178650 & 2.513340 & $\mathrm{H}$ & -1.114150 & -2.959370 & 8.580570 \\
\hline $\mathrm{H}$ & 2.377980 & -6.388180 & 3.874490 & $\mathrm{H}$ & -1.562140 & -1.223360 & 8.497050 \\
\hline 0 & 3.619070 & -4.778650 & 1.895540 & 0 & -1.625260 & -4.263090 & 6.029240 \\
\hline C & 4.810560 & -5.273690 & 2.494090 & C & -0.868430 & -5.345830 & 6.559980 \\
\hline $\mathrm{H}$ & 4.605650 & -5.781810 & 3.466560 & $\mathrm{H}$ & -0.309830 & -5.045230 & 7.478140 \\
\hline $\mathrm{H}$ & 5.239110 & -6.012420 & 1.785150 & $\mathrm{H}$ & -1.595850 & -6.141440 & 6.822100 \\
\hline $\mathrm{H}$ & 5.559260 & -4.465520 & 2.670460 & $\mathrm{H}$ & -0.140350 & -5.751570 & 5.819090 \\
\hline
\end{tabular}




\section{trans- $\alpha$}

$E_{P B E / S V P}=-6195.877350018$ Hartree $\mathrm{NImag}=0$

C

C

C

C

$\mathrm{N}$

C

C

$N$

H

H

$\mathrm{H}$

c

C

C

$\mathrm{Ni} \quad 1.007790$

N $\quad 2.453790$

C $\quad 2.261900$

C $\quad 3.540650$

C $\quad 4.508700$

C $\quad 3.816050$

C $\quad-2.456260$

C $\quad-1.800490$

C $\quad-2.489920$

C $\quad-1.520160$

C $\quad-0.243160$

N $\quad-0.438030$

C $\quad 1.010140$

C $\quad 4.462710$

$\mathrm{H} \quad-3.585290$

H $\quad-1.675970$

H $\quad-3.577910$

$\mathrm{H} \quad-1.658610$

H $\quad 5.596260$

H $\quad 3.681790$

H $\quad 3.664610$

H $\quad 5.586710$

C $\quad 0.992660$

C $\quad 5.952790$

C $\quad-3.934380$
$-0.271240$

$-0.460350$

4.293390

4.026410

$-0.502480 \quad 5.062100$

$-0.343560 \quad 6.391810$

$-0.177080 \quad 6.693430$

$-0.141250 \quad 5.631290$

$-0.239640 \quad 3.159440$

$-0.083210 \quad 3.356460$

$-0.046870 \quad 2.182490$

$-0.122870 \quad 0.829400$

$0.113240 \quad 2.210370$

$-0.010960 \quad 0.073140$

$0.128610 \quad 0.920800$

$-0.579560 \quad 2.975130$

$-0.022090 \quad 5.818720$

$-0.250150 \quad 0.404760$

$0.244920 \quad 0.632140$

$2.832510-2.183250$

$1.470440-2.161600$

$3.526100-2.181260$

$2.559630-2.149810$

$1.281000-2.135820$

$3.481030-2.182760$

$2.826580-2.152990$

$3.517190-2.109570$

$2.549370-2.039130$

$1.270760-2.076430$

$1.463600-2.145420$

$0.018010-1.959070$

$-1.417130 \quad-2.224720$

$-2.777190-2.305400$

$-3.466550-2.335110$

$-2.501040-2.246890$

$-1.225040-2.187780$

$0.013440-2.056810$

$-1.233320-2.174780$

$-2.509490-2.258320$

$-3.472250-2.353740$

$-2.779670-2.324210$

$-1.419880 \quad-2.229510$

$-3.429280-2.356450$

$0.028940-2.119320$

$2.693680-1.981410$

$4.604480-2.130620$

$-2.649530-2.262660$

$-4.557260-2.434050$

$-2.642570-2.236880$

$-4.550880-2.420970$

$4.614110-2.200750$

$2.700020-2.153020$

$4.976030-2.193790$

$0.028760-2.009510$

$-0.007200-1.835370$

\section{trans- $\beta$}

$E_{P B E / S V P}=-6195.875085082$ Hartree NImag $=0$

\begin{tabular}{|c|c|c|c|}
\hline $\mathrm{C}$ & -0.981650 & -0.177450 & 5.377800 \\
\hline$C$ & -0.318400 & -0.143580 & 6.622830 \\
\hline$c$ & -1.027280 & -0.209510 & 7.832860 \\
\hline C & -2.439700 & -0.307240 & 7.766670 \\
\hline 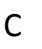 & -3.133180 & -0.370150 & 6.525510 \\
\hline C & -2.391290 & -0.299690 & 5.341690 \\
\hline $\mathrm{N}$ & -0.158200 & -0.099630 & 4.238740 \\
\hline$N$ & -0.777720 & -0.135480 & 3.120950 \\
\hline$C$ & -0.014120 & -0.059560 & 1.987600 \\
\hline$C$ & -0.512250 & -0.084140 & 0.678260 \\
\hline$N$ & 1.383910 & 0.058010 & 1.851230 \\
\hline $\mathrm{N}$ & 0.520710 & 0.013280 & -0.206910 \\
\hline$C$ & 1.640700 & 0.096840 & 0.520450 \\
\hline $\mathrm{H}$ & 0.781820 & -0.074560 & 6.621590 \\
\hline $\mathrm{H}$ & -2.887270 & -0.342750 & 4.359680 \\
\hline $\mathrm{H}$ & -1.561210 & -0.170420 & 0.369920 \\
\hline $\mathrm{H}$ & 2.656460 & 0.188440 & 0.110700 \\
\hline$C$ & 2.407660 & 0.125870 & 2.887740 \\
\hline$C$ & 1.736790 & 2.827450 & -2.491620 \\
\hline $\mathrm{N}$ & 1.925730 & 1.464020 & -2.474680 \\
\hline$C$ & 3.016910 & 3.514770 & -2.496460 \\
\hline $\mathrm{C}$ & 3.983880 & 2.543470 & -2.477490 \\
\hline$C$ & 3.288370 & 1.268410 & -2.460110 \\
\hline$C$ & 0.485230 & 3.481820 & -2.493770 \\
\hline$C$ & -0.768060 & 2.831470 & -2.484030 \\
\hline$C$ & -2.044300 & 3.526080 & -2.509860 \\
\hline$C$ & -3.016090 & 2.561200 & -2.482720 \\
\hline$C$ & -2.328380 & 1.281330 & -2.458450 \\
\hline $\mathrm{N}$ & -0.965020 & 1.469450 & -2.460730 \\
\hline $\mathrm{Ni}$ & 0.474510 & 0.020760 & -2.240250 \\
\hline $\mathrm{N}$ & 1.919350 & -1.426800 & -2.480960 \\
\hline$C$ & 1.722680 & -2.788740 & -2.509510 \\
\hline$C$ & 2.998620 & -3.483000 & -2.539730 \\
\hline$C$ & 3.970910 & -2.518210 & -2.504270 \\
\hline$C$ & 3.282910 & -1.238780 & -2.472930 \\
\hline$C$ & -2.982030 & 0.029720 & -2.442160 \\
\hline$C$ & -2.333000 & -1.224500 & -2.464590 \\
\hline$C$ & -3.027930 & -2.500020 & -2.503390 \\
\hline$C$ & -2.061640 & -3.470960 & -2.522030 \\
\hline$C$ & -0.781700 & -2.783580 & -2.501810 \\
\hline $\mathrm{N}$ & -0.970880 & -1.420320 & -2.469100 \\
\hline$C$ & 0.469050 & -3.438590 & -2.514620 \\
\hline$C$ & 3.935590 & 0.013540 & -2.437760 \\
\hline $\mathrm{H}$ & -4.103380 & 2.704740 & -2.486840 \\
\hline $\mathrm{H}$ & -2.180510 & 4.613570 & -2.552590 \\
\hline $\mathrm{H}$ & -4.116150 & -2.635580 & -2.527030 \\
\hline $\mathrm{H}$ & -2.204310 & -4.558050 & -2.550130 \\
\hline $\mathrm{H}$ & 5.058180 & -2.662410 & -2.511280 \\
\hline $\mathrm{H}$ & 3.134750 & -4.570190 & -2.590480 \\
\hline $\mathrm{H}$ & 3.159500 & 4.602180 & -2.513210 \\
\hline $\mathrm{H}$ & 5.072660 & 2.678060 & -2.489810 \\
\hline$C$ & 0.485670 & 4.976580 & -2.500470 \\
\hline$C$ & 5.426550 & 0.005850 & -2.336270 \\
\hline
\end{tabular}




\begin{tabular}{|c|c|c|c|c|c|c|c|}
\hline E & 1.014870 & -4.922650 & -2.423430 & C & -4.475220 & 0.028230 & -2.386570 \\
\hline F & 1.375280 & -5.700190 & -1.305730 & C & 0.469200 & -4.933420 & -2.528530 \\
\hline$C$ & 1.385850 & -7.104200 & -1.352530 & C & 0.835200 & -5.671960 & -1.386570 \\
\hline C & 1.030010 & -7.759330 & -2.543660 & C & 0.842950 & -7.076930 & -1.384000 \\
\hline C & 0.666220 & -7.010050 & -3.675300 & C & 0.479110 & -7.772080 & -2.549700 \\
\hline C & 0.662210 & -5.606440 & -3.603060 & C & 0.110250 & -7.062070 & -3.704800 \\
\hline $\mathrm{F}$ & 1.718220 & -5.103710 & -0.157800 & C & 0.108930 & -5.656790 & -3.681940 \\
\hline $\mathrm{F}$ & 1.726410 & -7.819060 & -0.276920 & $\mathrm{~F}$ & 1.186430 & -5.034740 & -0.263380 \\
\hline $\mathrm{F}$ & 1.037850 & -9.091690 & -2.599740 & $\mathrm{~F}$ & 1.188360 & -7.755770 & -0.286720 \\
\hline $\mathrm{F}$ & 0.333200 & -7.636070 & -4.807160 & $\mathrm{~F}$ & 0.483810 & -9.105500 & -2.559450 \\
\hline $\mathrm{F}$ & 0.313190 & -4.917640 & -4.694350 & $\mathrm{~F}$ & -0.230000 & -7.727090 & -4.811650 \\
\hline C & -4.459920 & -0.478170 & -0.615140 & $\mathrm{~F}$ & -0.244430 & -5.005350 & -4.794780 \\
\hline C & -5.837190 & -0.482300 & -0.347100 & C & -5.150940 & -0.327230 & -1.203540 \\
\hline C & -6.732320 & -0.013210 & -1.322940 & C & -6.553350 & -0.339270 & -1.124520 \\
\hline C & -6.240480 & 0.457310 & -2.552730 & C & -7.309750 & 0.011890 & -2.255870 \\
\hline C & -4.855960 & 0.449670 & -2.797230 & C & -6.662840 & 0.372590 & -3.450210 \\
\hline $\mathrm{F}$ & -3.634900 & -0.918060 & 0.345410 & C & -5.258500 & 0.377030 & -3.503190 \\
\hline $\mathrm{F}$ & -6.299650 & -0.921490 & 0.830440 & $\mathrm{~F}$ & -4.451790 & -0.661630 & -0.110040 \\
\hline $\mathrm{F}$ & -8.043600 & -0.010440 & -1.081780 & $\mathrm{~F}$ & -7.171950 & -0.675050 & 0.010920 \\
\hline $\mathrm{F}$ & -7.091500 & 0.903320 & -3.479830 & $\mathrm{~F}$ & -8.641760 & 0.004520 & -2.195160 \\
\hline $\mathrm{F}$ & -4.422550 & 0.901550 & -3.978120 & $\mathrm{~F}$ & -7.385160 & 0.703480 & -4.522920 \\
\hline C & 6.584060 & 0.322420 & -0.786300 & $\mathrm{~F}$ & -4.668980 & 0.723300 & -4.652160 \\
\hline C & 7.980770 & 0.298560 & -0.644390 & C & 6.065490 & 0.284120 & -1.113680 \\
\hline C & 8.779650 & -0.021210 & -1.755930 & C & 7.462260 & 0.248090 & -0.977350 \\
\hline C & 8.178650 & -0.314280 & -2.992120 & C & 8.253630 & -0.068610 & -2.095260 \\
\hline C & 6.778250 & -0.283850 & -3.106210 & C & 7.645030 & -0.347030 & -3.331120 \\
\hline $\mathrm{F}$ & 5.844980 & 0.624840 & 0.294470 & C & 6.244300 & -0.304600 & -3.439020 \\
\hline $\mathrm{F}$ & 8.552790 & 0.567840 & 0.532690 & $\mathrm{~F}$ & 5.332300 & 0.583570 & -0.026530 \\
\hline $\mathrm{F}$ & 10.107170 & -0.049340 & -1.635930 & $\mathrm{~F}$ & 8.041150 & 0.503120 & 0.199290 \\
\hline $\mathrm{F}$ & 8.938900 & -0.617790 & -4.046120 & $\mathrm{~F}$ & 9.581230 & -0.108120 & -1.981380 \\
\hline $\mathrm{F}$ & 6.234110 & -0.567360 & -4.293450 & $\mathrm{~F}$ & 8.398470 & -0.649070 & -4.390190 \\
\hline C & 1.344340 & 5.698010 & -3.350940 & $\mathrm{~F}$ & 5.691950 & -0.575970 & -4.624980 \\
\hline C & 1.345630 & 7.103230 & -3.375460 & C & 0.844710 & 5.704740 & -3.651310 \\
\hline C & 0.987540 & 7.815230 & -2.218260 & C & 0.844040 & 7.110090 & -3.668150 \\
\hline C & 0.633090 & 7.121780 & -1.048700 & C & 0.478100 & 7.815360 & -2.509210 \\
\hline C & 0.640400 & 5.716750 & -1.048960 & C & 0.116130 & 7.115430 & -1.345800 \\
\hline $\mathrm{F}$ & 1.688210 & 5.045020 & -4.465730 & C & 0.123410 & 5.710450 & -1.354260 \\
\hline $\mathrm{F}$ & 1.678730 & 7.766310 & -4.485690 & $\mathrm{~F}$ & 1.195800 & 5.058000 & -4.767610 \\
\hline $\mathrm{F}$ & 0.984530 & 9.148760 & -2.229510 & $\mathrm{~F}$ & 1.182900 & 7.779680 & -4.772650 \\
\hline $\mathrm{F}$ & 0.298140 & 7.802430 & 0.050960 & $\mathrm{~F}$ & 0.474480 & 9.148870 & -2.513140 \\
\hline $\mathrm{F}$ & 0.302460 & 5.082190 & 0.079940 & $\mathrm{~F}$ & -0.225720 & 7.789900 & -0.244610 \\
\hline C & 2.410880 & 0.240870 & 3.404810 & $\mathrm{~F}$ & -0.223560 & 5.068620 & -0.232590 \\
\hline $\mathrm{H}$ & 2.078250 & 1.111830 & 4.006590 & $\mathrm{H}$ & 3.394560 & 0.213130 & 2.386720 \\
\hline $\mathrm{H}$ & 2.326440 & -0.672010 & 4.030200 & $\mathrm{H}$ & 2.379570 & -0.784120 & 3.518570 \\
\hline $\mathrm{H}$ & 3.466090 & 0.383350 & 3.097020 & $\mathrm{H}$ & 2.235060 & 1.000100 & 3.545360 \\
\hline C & -2.597780 & -0.072260 & 8.127380 & C & -4.633690 & -0.531310 & 6.515150 \\
\hline $\mathrm{H}$ & -1.535230 & -0.379180 & 8.225620 & $\mathrm{H}$ & -5.147740 & 0.356910 & 6.947360 \\
\hline $\mathrm{H}$ & -2.673250 & 0.971080 & 8.512830 & $\mathrm{H}$ & -5.010390 & -0.670890 & 5.480720 \\
\hline $\mathrm{H}$ & -3.217740 & -0.711060 & 8.791580 & $\mathrm{H}$ & -4.945870 & -1.404590 & 7.128650 \\
\hline C & -6.373540 & -0.731750 & 4.788640 & C & -0.314830 & -0.208370 & 9.163850 \\
\hline $\mathrm{H}$ & -6.999230 & 0.118850 & 5.140640 & $\mathrm{H}$ & 0.737890 & -0.542660 & 9.050800 \\
\hline $\mathrm{H}$ & -6.555280 & -0.860890 & 3.701280 & $\mathrm{H}$ & -0.288340 & 0.807550 & 9.621740 \\
\hline $\mathrm{H}$ & -6.744340 & -1.635910 & 5.320160 & $\mathrm{H}$ & -0.826400 & -0.878140 & 9.887660 \\
\hline 0 & -5.357870 & -0.436050 & 7.415040 & 0 & -3.175370 & -0.428890 & 8.924850 \\
\hline C & -5.833140 & 0.794900 & 7.947130 & C & -3.497490 & 0.784270 & 9.594480 \\
\hline $\mathrm{H}$ & -6.329040 & 1.416390 & 7.163570 & $\mathrm{H}$ & -4.093100 & 1.467060 & 8.943050 \\
\hline $\mathrm{H}$ & -6.577390 & 0.536270 & 8.728320 & $\mathrm{H}$ & -4.106360 & 0.505780 & 10.479380 \\
\hline $\mathrm{H}$ & -5.015760 & 1.396040 & 8.411420 & $\mathrm{H}$ & -2.586650 & 1.328370 & 9.939810 \\
\hline
\end{tabular}




\section{I.6.8 1-Methyl-3',5'-di-tert-butyl-4'-methoxy-5-phenylazoimidazole $\cdot$ NiTPPF $_{20}(2 \mathrm{~g})$}

\begin{tabular}{|c|c|c|c|}
\hline \multicolumn{4}{|c|}{ cis- $\alpha$} \\
\hline \multicolumn{4}{|c|}{$E_{P B E / S V P}=-6431.179007152$ Hartree } \\
\hline \multicolumn{4}{|c|}{ NImag $=0$} \\
\hline C & 0.582160 & -1.767290 & 3.593890 \\
\hline C & 0.467340 & -3.164360 & 3.483930 \\
\hline $\mathrm{C}$ & 1.511380 & -3.950880 & 2.972150 \\
\hline C & 2.745880 & -3.291400 & 2.656520 \\
\hline C & 2.876600 & -1.863700 & 2.704000 \\
\hline C & 1.770620 & -1.137730 & 3.182160 \\
\hline $\mathrm{N}$ & -0.442120 & -1.074440 & 4.284680 \\
\hline $\mathrm{N}$ & -0.896630 & 0.052630 & 3.943810 \\
\hline C & -0.732790 & 0.663830 & 2.704270 \\
\hline C & -0.673770 & 0.246710 & 1.362890 \\
\hline $\mathrm{N}$ & -1.015090 & 2.034910 & 2.647100 \\
\hline $\mathrm{N}$ & -0.910760 & 1.319740 & 0.549230 \\
\hline C & -1.086700 & 2.384650 & 1.342160 \\
\hline $\mathrm{H}$ & -0.478660 & -3.614390 & 3.811720 \\
\hline $\mathrm{H}$ & 1.833390 & -0.045780 & 3.272460 \\
\hline $\mathrm{H}$ & -0.523170 & -0.764530 & 0.967120 \\
\hline $\mathrm{H}$ & -1.261890 & 3.413700 & 1.001100 \\
\hline C & -0.144510 & 4.285790 & -1.754560 \\
\hline$N$ & 0.114640 & 2.936850 & -1.852770 \\
\hline C & 1.063160 & 5.053270 & -2.003430 \\
\hline C & 2.054740 & 4.145520 & -2.265050 \\
\hline C & 1.455260 & 2.826970 & -2.146280 \\
\hline C & -1.402600 & 4.859900 & -1.469210 \\
\hline C & -2.610000 & 4.140840 & -1.321380 \\
\hline C & -3.909780 & 4.761180 & -1.130510 \\
\hline C & -4.824410 & 3.741540 & -1.080720 \\
\hline C & -4.077000 & 2.505470 & -1.229510 \\
\hline$N$ & -2.734300 & 2.771430 & -1.377300 \\
\hline $\mathrm{Ni}$ & -1.197510 & 1.396060 & -1.472850 \\
\hline$N$ & 0.256530 & 0.054640 & -2.029580 \\
\hline C & 0.135700 & -1.316670 & -2.059500 \\
\hline C & 1.435960 & -1.935580 & -2.247070 \\
\hline C & 2.342980 & -0.913720 & -2.348210 \\
\hline C & 1.592190 & 0.324830 & -2.221140 \\
\hline C & -4.665200 & 1.222720 & -1.256860 \\
\hline C & -3.959690 & 0.013890 & -1.442970 \\
\hline C & -4.591740 & -1.292630 & -1.504110 \\
\hline C & -3.589650 & -2.203030 & -1.707210 \\
\hline C & -2.348110 & -1.449600 & -1.756210 \\
\hline$N$ & -2.596800 & -0.105210 & -1.600520 \\
\hline C & -1.075480 & -2.033570 & -1.949010 \\
\hline C & 2.164550 & 1.615090 & -2.308080 \\
\hline $\mathrm{H}$ & -5.913080 & 3.821320 & -0.973110 \\
\hline $\mathrm{H}$ & -4.102430 & 5.839600 & -1.073930 \\
\hline $\mathrm{H}$ & -5.665970 & -1.490150 & -1.407210 \\
\hline $\mathrm{H}$ & -3.686660 & -3.288730 & -1.826440 \\
\hline $\mathrm{H}$ & 3.427220 & -0.995330 & -2.490800 \\
\hline $\mathrm{H}$ & 1.631750 & -3.014130 & -2.288700 \\
\hline $\mathrm{H}$ & 1.140110 & 6.147380 & -1.995630 \\
\hline $\mathrm{H}$ & 3.099590 & 4.353780 & -2.525040 \\
\hline C & -1.476730 & 6.344320 & -1.313060 \\
\hline
\end{tabular}

\section{cis- $\beta$}

$E_{P B E / S V P}=-6431.168756492$ Hartree NImag $=0$

$\begin{array}{lrrr}\text { C } & 0.282370 & 0.086750 & 4.212670 \\ \text { C } & -0.455940 & -0.187160 & 5.377430 \\ \text { C } & -0.776870 & -1.497910 & 5.774050 \\ \text { C } & -0.173010 & -2.562650 & 5.026290 \\ \text { C } & 0.560200 & -2.320150 & 3.815620 \\ \text { C } & 0.752500 & -0.985800 & 3.425560 \\ \text { N } & 0.651940 & 1.431540 & 3.980160\end{array}$

$\begin{array}{llll}\mathrm{N} & 0.788350 & 1.954320 & 2.835690\end{array}$

$\begin{array}{llll}\text { C } & 0.285750 & 1.446540 & 1.637370\end{array}$

$\begin{array}{llll}\text { C } & 0.874780 & 1.577060 & 0.374680\end{array}$

N $\quad-0.997450 \quad 0.925640 \quad 1.395770$

N $\quad 0.008440 \quad 1.122810 \quad-0.583120$

$\begin{array}{llll}\text { C } & -1.111260 & 0.766090 & 0.048020\end{array}$

$\begin{array}{llll}\mathrm{H} & -0.804110 & 0.680450 & 5.954770\end{array}$

$\begin{array}{llll}\mathrm{H} & 1.328750 & -0.753740 & 2.521470\end{array}$

$\begin{array}{llll}\mathrm{H} & 1.880680 & 1.946440 & 0.142470\end{array}$

$\begin{array}{llll}H & -2.042180 & 0.444770 & -0.437830\end{array}$

C $\quad 1.546330 \quad 3.915530 \quad-2.824790$

$\mathrm{N} \quad \begin{array}{llll}\mathrm{N} & 1.700920 & 2.551660 & -2.714780\end{array}$

C $\quad 2.839030 \quad 4.575580 \quad-2.761940$

$\begin{array}{llll}\text { C } & 3.775280 & 3.589200 & -2.597410\end{array}$

$\begin{array}{llll}\text { C } & 3.050900 & 2.330070 & -2.564500\end{array}$

C $\quad 0.314910 \quad 4.596260 \quad-2.947740$

C $\quad-0.952590 \quad 3.974850 \quad-2.982100$

C $\quad-2.212480 \quad 4.694540 \quad-3.063960$

C $\quad-3.204960 \quad 3.750000 \quad-3.050120$

C $\quad-2.543780 \quad 2.456990 \quad-2.994050$

N $\quad-1.177950 \quad 2.618090 \quad-2.947370$

$\mathrm{Ni} \quad 0.218610 \quad 1.140300 \quad-2.613060$

N $\quad 1.648250 \quad-0.331930 \quad-2.735580$

C $\quad 1.430470 \quad-1.687300 \quad-2.828390$

C $\quad 2.678250 \quad-2.412450 \quad-2.659380$

C $\quad 3.650230 \quad-1.471880 \quad-2.440880$

$\begin{array}{llll}\text { C } & 2.996630 & -0.175060 & -2.512900\end{array}$

C $\quad-3.217260 \quad 1.216510 \quad-3.029710$

C $\quad-2.585540 \quad-0.045750 \quad-3.095140$

C $\quad-3.291790 \quad-1.302240 \quad-3.273370$

C $\quad-2.336620 \quad-2.285570 \quad-3.308940$

C $\quad-1.054570 \quad-1.625350 \quad-3.135950$

$\mathrm{N} \quad-1.228230 \quad-0.264430 \quad-3.021350$

C $\quad 0.180600 \quad-2.303540 \quad-3.051650$

C $\quad 3.666460 \quad 1.065950 \quad-2.414790$

$\mathrm{H} \quad-4.288360 \quad 3.915610 \quad-3.094910$

$\begin{array}{llll}H & -2.324490 & 5.783730 & -3.128250\end{array}$

H $\quad-4.377530 \quad-1.415950 \quad-3.382860$

H $\quad-2.488180 \quad-3.364130 \quad-3.439870$

H $\quad 4.718080 \quad-1.644410 \quad-2.260980$

H $\quad 2.795310 \quad-3.502460 \quad-2.702020$

$\begin{array}{llll}H & 3.009750 & 5.656740 & -2.830830\end{array}$

$\begin{array}{llll}\mathrm{H} & 4.863630 & 3.703490 & -2.523360\end{array}$

$\begin{array}{llll}\text { C } & 0.361360 & 6.087720 & -3.044270\end{array}$ 


\begin{tabular}{|c|c|c|c|c|c|c|c|}
\hline E & 3.626980 & 1.696430 & -2.601030 & C & 5.132260 & 1.051380 & -2.130480 \\
\hline E & -6.149530 & 1.135960 & -1.103340 & C & -4.710820 & 1.227460 & -2.987810 \\
\hline$C$ & -1.028250 & -3.521600 & -2.071230 & C & 0.146090 & -3.793810 & -3.175520 \\
\hline C & -1.298990 & -4.350900 & -0.966600 & C & 0.185720 & -4.620950 & -2.039310 \\
\hline C & -1.336480 & -5.749560 & -1.076610 & C & 0.118840 & -6.020030 & -2.131370 \\
\hline C & -1.078030 & -6.351230 & -2.319680 & C & 0.009150 & -6.620970 & -3.396880 \\
\hline C & -0.792400 & -5.551250 & -3.439060 & C & -0.034230 & -5.820670 & -4.551860 \\
\hline C & -0.765930 & -4.152610 & -3.303340 & C & 0.038750 & -4.422080 & -4.430360 \\
\hline $\mathrm{F}$ & -1.521340 & -3.803300 & 0.238740 & $\mathrm{~F}$ & 0.282560 & -4.072380 & -0.820200 \\
\hline $\mathrm{F}$ & -1.592530 & -6.512560 & -0.009070 & $\mathrm{~F}$ & 0.152270 & -6.776930 & -1.029880 \\
\hline $\mathrm{F}$ & -1.103120 & -7.678950 & -2.436050 & $\mathrm{~F}$ & -0.057470 & -7.948390 & -3.502150 \\
\hline $\mathrm{F}$ & -0.556650 & -6.123250 & -4.621970 & $\mathrm{~F}$ & -0.143010 & -6.393100 & -5.752970 \\
\hline $\mathrm{F}$ & -0.498740 & -3.419590 & -4.388090 & $\mathrm{~F}$ & -0.006230 & -3.685880 & -5.543910 \\
\hline C & -6.774970 & 1.359240 & 0.138030 & C & -5.393790 & 0.859380 & -1.813110 \\
\hline C & -8.167040 & 1.254540 & 0.295670 & C & -6.795550 & 0.846830 & -1.737140 \\
\hline C & -8.966020 & 0.924970 & -0.812300 & C & -7.547180 & 1.216050 & -2.866100 \\
\hline C & -8.370370 & 0.703530 & -2.065550 & C & -6.894770 & 1.588880 & -4.053750 \\
\hline C & -6.976290 & 0.814200 & -2.197260 & C & -5.489770 & 1.593970 & -4.101590 \\
\hline $\mathrm{F}$ & -6.040120 & 1.674970 & 1.210650 & $\mathrm{~F}$ & -4.699280 & 0.503800 & -0.716800 \\
\hline $\mathrm{F}$ & -8.733660 & 1.459470 & 1.487640 & $\mathrm{~F}$ & -7.417330 & 0.496750 & -0.607700 \\
\hline $\mathrm{F}$ & -10.288140 & 0.821920 & -0.674260 & $\mathrm{~F}$ & -8.878960 & 1.210100 & -2.810220 \\
\hline $\mathrm{F}$ & -9.129500 & 0.393120 & -3.118920 & $\mathrm{~F}$ & -7.612700 & 1.932780 & -5.124580 \\
\hline $\mathrm{F}$ & -6.437620 & 0.600930 & -3.402230 & $\mathrm{~F}$ & -4.896130 & 1.953490 & -5.243500 \\
\hline C & 4.546500 & 2.196900 & -1.658460 & C & 5.626690 & 1.527740 & -0.899630 \\
\hline C & 5.926060 & 2.246440 & -1.915430 & C & 6.998130 & 1.526200 & -0.598260 \\
\hline C & 6.415720 & 1.797420 & -3.153530 & C & 7.911740 & 1.032840 & -1.545320 \\
\hline C & 5.523930 & 1.300010 & -4.118880 & C & 7.449560 & 0.551060 & -2.781710 \\
\hline C & 4.148170 & 1.261380 & -3.837030 & C & 6.072920 & 0.570770 & -3.062390 \\
\hline $\mathrm{F}$ & 4.117330 & 2.629040 & -0.464500 & $\mathrm{~F}$ & 4.779380 & 1.995490 & 0.027360 \\
\hline $\mathrm{F}$ & 6.772240 & 2.711410 & -0.991630 & $\mathrm{~F}$ & 7.439050 & 1.979980 & 0.577140 \\
\hline $\mathrm{F}$ & 7.723300 & 1.839060 & -3.410270 & $\mathrm{~F}$ & 9.216080 & 1.021780 & -1.269590 \\
\hline $\mathrm{F}$ & 5.987640 & 0.875660 & -5.296330 & $\mathrm{~F}$ & 8.319990 & 0.085330 & -3.680620 \\
\hline $\mathrm{F}$ & 3.327580 & 0.790010 & -4.780790 & $\mathrm{~F}$ & 5.665740 & 0.117590 & -4.252680 \\
\hline C & -1.438060 & 7.209230 & -2.422730 & C & 0.806400 & 6.723140 & -4.219990 \\
\hline C & -1.545570 & 8.602820 & -2.277230 & C & 0.873730 & 8.122370 & -4.327070 \\
\hline C & -1.687760 & 9.155800 & -0.993090 & C & 0.488050 & 8.917840 & -3.234740 \\
\hline C & -1.724520 & 8.316370 & 0.133990 & C & 0.035870 & 8.311650 & -2.050180 \\
\hline C & -1.616500 & 6.927480 & -0.040250 & C & -0.024070 & 6.910240 & -1.968350 \\
\hline $\mathrm{F}$ & -1.304280 & 6.708940 & -3.654340 & $\mathrm{~F}$ & 1.180100 & 5.990790 & -5.274050 \\
\hline $\mathrm{F}$ & -1.517730 & 9.401050 & -3.346170 & $\mathrm{~F}$ & 1.299190 & 8.698220 & -5.454480 \\
\hline $\mathrm{F}$ & -1.789330 & 10.476370 & -0.842910 & $\mathrm{~F}$ & 0.548870 & 10.247010 & -3.322560 \\
\hline $\mathrm{F}$ & -1.857510 & 8.843730 & 1.353740 & $\mathrm{~F}$ & -0.326640 & 9.068540 & -1.011350 \\
\hline $\mathrm{F}$ & -1.650770 & 6.148510 & 1.054480 & $\mathrm{~F}$ & -0.459850 & 6.363610 & -0.827900 \\
\hline C & -1.184260 & 2.905520 & 3.799510 & C & -2.091780 & 0.739360 & 2.338020 \\
\hline $\mathrm{H}$ & -1.761880 & 2.356620 & 4.571290 & $\mathrm{H}$ & -2.000720 & -0.229100 & 2.873590 \\
\hline $\mathrm{H}$ & -0.200350 & 3.192090 & 4.230080 & $\mathrm{H}$ & -2.086720 & 1.559720 & 3.084870 \\
\hline $\mathrm{H}$ & -1.726660 & 3.819100 & 3.483610 & $\mathrm{H}$ & -3.047700 & 0.770790 & 1.775830 \\
\hline C & 4.131400 & -1.060490 & 2.254690 & C & -1.760570 & -1.678300 & 6.966900 \\
\hline C & 1.285740 & -5.470650 & 2.746080 & C & 1.094630 & -3.465050 & 2.913300 \\
\hline O & 3.784610 & -4.094880 & 2.230090 & 0 & -0.318110 & -3.878820 & 5.407370 \\
\hline C & 4.870570 & -4.380440 & 3.102690 & C & 0.409440 & -4.361010 & 6.533220 \\
\hline $\mathrm{H}$ & 4.592530 & -4.231080 & 4.169760 & $\mathrm{H}$ & -0.243040 & -4.437200 & 7.432910 \\
\hline $\mathrm{H}$ & 5.156020 & -5.444620 & 2.949960 & $\mathrm{H}$ & 0.776260 & -5.381700 & 6.288550 \\
\hline $\mathrm{H}$ & 5.758860 & -3.751670 & 2.862640 & $\mathrm{H}$ & 1.281550 & -3.710880 & 6.766510 \\
\hline C & 4.782270 & -1.655500 & 0.981420 & C & -0.100640 & -4.314310 & 2.410120 \\
\hline $\mathrm{H}$ & 4.051840 & -1.657820 & 0.141430 & $\mathrm{H}$ & -0.803300 & -3.689480 & 1.816180 \\
\hline $\mathrm{H}$ & 5.647290 & -1.023280 & 0.679690 & $\mathrm{H}$ & 0.258160 & -5.130070 & 1.743600 \\
\hline $\mathrm{H}$ & 5.137850 & -2.694400 & 1.108790 & $\mathrm{H}$ & -0.656890 & -4.765090 & 3.257500 \\
\hline
\end{tabular}




$\begin{array}{lrrrrrrr}\mathrm{C} & 3.755240 & 0.397540 & 1.886170 & \mathrm{C} & 1.838670 & -2.909980 & 1.677600 \\ \mathrm{H} & 4.639350 & 0.904080 & 1.444060 & \mathrm{H} & 2.181290 & -3.758210 & 1.048420 \\ \mathrm{H} & 2.934850 & 0.440730 & 1.136350 & \mathrm{H} & 1.191220 & -2.277570 & 1.033750 \\ \mathrm{H} & 3.454580 & 1.002730 & 2.769090 & \mathrm{H} & 2.738130 & -2.320380 & 1.962340 \\ \mathrm{C} & 5.153090 & -0.968080 & 3.417380 & \mathrm{C} & 2.104220 & -4.369530 & 3.663460 \\ \mathrm{H} & 4.690040 & -0.479550 & 4.303010 & \mathrm{H} & 2.893350 & -3.766370 & 4.164910 \\ \mathrm{H} & 5.533630 & -1.955920 & 3.743920 & \mathrm{H} & 1.614630 & -5.010990 & 4.419760 \\ \mathrm{H} & 6.028270 & -0.353450 & 3.107080 & \mathrm{H} & 2.608400 & -5.045010 & 2.936890 \\ \mathrm{C} & 1.582530 & -5.822120 & 1.266340 & \mathrm{C} & -2.670970 & -2.920260 & 6.802470 \\ \mathrm{H} & 0.928490 & -5.226320 & 0.592990 & \mathrm{H} & -2.124840 & -3.880940 & 6.815070 \\ \mathrm{H} & 2.637680 & -5.612090 & 1.001270 & \mathrm{H} & -3.418200 & -2.939390 & 7.626970 \\ \mathrm{H} & 1.371950 & -6.899130 & 1.079700 & \mathrm{H} & -3.228830 & -2.872810 & 5.841320 \\ \mathrm{C} & -0.180300 & -5.871720 & 3.028650 & \mathrm{C} & -2.725200 & -0.465510 & 7.056520 \\ \mathrm{H} & -0.312870 & -6.950380 & 2.793780 & \mathrm{H} & -3.493770 & -0.667760 & 7.833800 \\ \mathrm{H} & -0.457820 & -5.729280 & 4.095890 & \mathrm{H} & -2.214290 & 0.475750 & 7.350440 \\ \mathrm{H} & -0.897080 & -5.306920 & 2.396390 & \mathrm{H} & -3.254150 & -0.289980 & 6.093330 \\ \mathrm{C} & 2.169520 & -6.326550 & 3.686140 & \mathrm{C} & -0.984170 & -1.731870 & 8.307380 \\ \mathrm{H} & 1.892380 & -7.399930 & 3.585050 & \mathrm{H} & -0.283030 & -2.588360 & 8.365070 \\ \mathrm{H} & 3.247480 & -6.242980 & 3.451310 & \mathrm{H} & -0.390070 & -0.802860 & 8.452570 \\ \mathrm{H} & 2.016950 & -6.036950 & 4.749690 & \mathrm{H} & -1.695920 & -1.820100 & 9.159100\end{array}$




\section{trans- $\alpha$}

$E_{P B E} / \mathrm{SVP}=-6431.198221422$ Hartree $\mathrm{NImag}=0$

\begin{tabular}{|c|c|c|c|}
\hline C & -1.967860 & -0.274390 & 3.414740 \\
\hline C & -3.337120 & -0.531850 & 3.208270 \\
\hline C & -4.243910 & -0.605150 & 4.275260 \\
\hline C & -3.728440 & -0.358010 & 5.590580 \\
\hline C & -2.319180 & -0.224020 & 5.847250 \\
\hline C & -1.474330 & -0.157910 & 4.728810 \\
\hline$N$ & -1.191970 & -0.198730 & 2.250670 \\
\hline $\mathrm{N}$ & 0.061460 & -0.011420 & 2.414290 \\
\hline C & 0.760310 & 0.065750 & 1.231390 \\
\hline C & 0.407450 & 0.023450 & -0.122170 \\
\hline $\mathrm{N}$ & 2.146560 & 0.230790 & 1.255370 \\
\hline$N$ & 1.534640 & 0.156010 & -0.879860 \\
\hline C & 2.567040 & 0.277670 & -0.034400 \\
\hline $\mathrm{H}$ & -3.652460 & -0.664630 & 2.164510 \\
\hline $\mathrm{H}$ & -0.390130 & -0.026540 & 4.839010 \\
\hline $\mathrm{H}$ & -0.595450 & -0.092310 & -0.547280 \\
\hline $\mathrm{H}$ & 3.619380 & 0.398250 & -0.325700 \\
\hline C & 2.897820 & 2.974070 & -3.097580 \\
\hline $\mathrm{N}$ & 3.073620 & 1.609080 & -3.077050 \\
\hline C & 4.183810 & 3.649420 & -3.062190 \\
\hline C & 5.140670 & 2.668810 & -3.016290 \\
\hline C & 4.433030 & 1.400300 & -3.023550 \\
\hline C & 1.653020 & 3.639860 & -3.137730 \\
\hline C & 0.393780 & 3.001980 & -3.166430 \\
\hline C & -0.874830 & 3.709570 & -3.211470 \\
\hline C & -1.856420 & 2.754160 & -3.206620 \\
\hline C & -1.181660 & 1.467280 & -3.179680 \\
\hline$N$ & 0.183240 & 1.641860 & -3.158620 \\
\hline $\mathrm{Ni}$ & 1.605910 & 0.177750 & -2.907850 \\
\hline$N$ & 3.039270 & -1.281480 & -3.113160 \\
\hline C & 2.830730 & -2.640700 & -3.167560 \\
\hline C & 4.100870 & -3.346440 & -3.172050 \\
\hline C & 5.080390 & -2.391680 & -3.094940 \\
\hline C & 4.403500 & -1.106250 & -3.063860 \\
\hline C & -1.847490 & 0.221630 & -3.179820 \\
\hline C & -1.210110 & -1.038380 & -3.208190 \\
\hline C & -1.916650 & -2.306650 & -3.265180 \\
\hline C & -0.959440 & -3.286670 & -3.276990 \\
\hline C & 0.326780 & -2.611850 & -3.231380 \\
\hline$N$ & 0.150250 & -1.247140 & -3.195260 \\
\hline $\mathrm{C}$ & 1.571540 & -3.278670 & -3.216870 \\
\hline C & 5.066920 & 0.138870 & -2.996420 \\
\hline $\mathrm{H}$ & -2.942040 & 2.908580 & -3.228030 \\
\hline $\mathrm{H}$ & -0.999310 & 4.798730 & -3.247980 \\
\hline $\mathrm{H}$ & -3.005790 & -2.431240 & -3.304280 \\
\hline $\mathrm{H}$ & -1.112450 & -4.372110 & -3.312850 \\
\hline $\mathrm{H}$ & 6.166010 & -2.546220 & -3.072770 \\
\hline $\mathrm{H}$ & 4.229010 & -4.433860 & -3.236090 \\
\hline $\mathrm{H}$ & 4.336620 & 4.735590 & -3.073300 \\
\hline $\mathrm{H}$ & 6.230560 & 2.793030 & -2.995500 \\
\hline $\mathrm{C}$ & 1.668380 & 5.134880 & -3.142140 \\
\hline C & 6.555520 & 0.115160 & -2.868710 \\
\hline C & -3.341560 & 0.234750 & -3.131840 \\
\hline
\end{tabular}

\section{trans- $\beta$}

$E_{P B E / S V P}=-6431.195810712$ Hartree NImag $=0$

\begin{tabular}{|c|c|c|c|}
\hline C & -0.646760 & -0.127610 & 4.250850 \\
\hline C & -0.010610 & -0.031820 & 5.499610 \\
\hline C & -0.714670 & -0.110130 & 6.715680 \\
\hline C & -2.137980 & -0.234900 & 6.621310 \\
\hline C & -2.803320 & -0.458110 & 5.364010 \\
\hline C & -2.033510 & -0.378570 & 4.198500 \\
\hline$N$ & 0.184870 & -0.008450 & 3.121450 \\
\hline$N$ & -0.421350 & -0.075120 & 1.997900 \\
\hline C & 0.346040 & 0.027520 & 0.869380 \\
\hline C & -0.145780 & -0.001680 & -0.442000 \\
\hline$N$ & 1.741510 & 0.176840 & 0.739730 \\
\hline$N$ & 0.888490 & 0.123130 & -1.322310 \\
\hline C & 2.003370 & 0.227000 & -0.589980 \\
\hline $\mathrm{H}$ & 1.079840 & 0.099270 & 5.481580 \\
\hline $\mathrm{H}$ & -2.476780 & -0.505100 & 3.203010 \\
\hline $\mathrm{H}$ & -1.191600 & -0.107300 & -0.754840 \\
\hline $\mathrm{H}$ & 3.019160 & 0.337490 & -0.994930 \\
\hline C & 2.758850 & 0.256910 & 1.781430 \\
\hline C & 2.131290 & 2.918670 & -3.623170 \\
\hline$N$ & 2.308040 & 1.553760 & -3.596930 \\
\hline C & 3.417530 & 3.594480 & -3.630470 \\
\hline C & 4.375780 & 2.614670 & -3.603340 \\
\hline C & 3.668870 & 1.346070 & -3.579220 \\
\hline C & 0.885500 & 3.583990 & -3.631080 \\
\hline C & -0.373400 & 2.944560 & -3.616290 \\
\hline C & -1.643610 & 3.650030 & -3.644960 \\
\hline C & -2.623690 & 2.693970 & -3.607680 \\
\hline C & -1.947060 & 1.408310 & -3.575400 \\
\hline $\mathrm{N}$ & -0.582150 & 1.584520 & -3.582730 \\
\hline $\mathrm{Ni}$ & 0.844610 & 0.124420 & -3.355130 \\
\hline $\mathrm{N}$ & 2.275990 & -1.337170 & -3.588780 \\
\hline C & 2.067470 & -2.697390 & -3.611660 \\
\hline C & 3.337360 & -3.402860 & -3.638280 \\
\hline C & 4.317980 & -2.446440 & -3.605710 \\
\hline C & 3.641010 & -1.160960 & -3.579850 \\
\hline C & -2.611650 & 0.162620 & -3.548110 \\
\hline C & -1.974040 & -1.097430 & -3.566670 \\
\hline C & -2.680470 & -2.366810 & -3.596080 \\
\hline C & -1.722880 & -3.346440 & -3.613860 \\
\hline C & -0.436780 & -2.670380 & -3.601620 \\
\hline$N$ & -0.613740 & -1.305370 & -3.575550 \\
\hline C & 0.808190 & -3.336330 & -3.613180 \\
\hline C & 4.304620 & 0.085600 & -3.549190 \\
\hline $\mathrm{H}$ & -3.709660 & 2.847070 & -3.610300 \\
\hline $\mathrm{H}$ & -1.770530 & 4.738280 & -3.696070 \\
\hline $\mathrm{H}$ & -3.769990 & -2.492490 & -3.614650 \\
\hline $\mathrm{H}$ & -1.875210 & -4.432370 & -3.635990 \\
\hline $\mathrm{H}$ & 5.403990 & -2.599980 & -3.611350 \\
\hline $\mathrm{H}$ & 3.464070 & -4.491370 & -3.684720 \\
\hline $\mathrm{H}$ & 3.569810 & 4.680460 & -3.654110 \\
\hline $\mathrm{H}$ & 5.465760 & 2.739340 & -3.614520 \\
\hline C & 0.898480 & 5.078590 & -3.650430 \\
\hline C & 5.795250 & 0.065270 & -3.444150 \\
\hline
\end{tabular}




\begin{tabular}{|c|c|c|c|}
\hline C & 1.558770 & -4.773010 & -3.243100 \\
\hline C & 1.911180 & -5.524590 & -2.105090 \\
\hline C & 1.909990 & -6.929460 & -2.115140 \\
\hline C & 1.547980 & -7.612220 & -3.288660 \\
\hline C & 1.191210 & -6.889540 & -4.439640 \\
\hline C & 1.200250 & -5.484560 & -4.404640 \\
\hline $\mathrm{F}$ & 2.258440 & -4.900930 & -0.972970 \\
\hline $\mathrm{F}$ & 2.244740 & -7.619750 & -1.021650 \\
\hline $\mathrm{F}$ & 1.543500 & -8.945590 & -3.309860 \\
\hline $\mathrm{F}$ & 0.852810 & -7.542430 & -5.554410 \\
\hline $\mathrm{F}$ & 0.859400 & -4.821750 & -5.514650 \\
\hline C & -4.031460 & -0.102940 & -1.951070 \\
\hline C & -5.434270 & -0.093760 & -1.884820 \\
\hline C & -6.178210 & 0.262110 & -3.022580 \\
\hline C & -5.517160 & 0.602490 & -4.214880 \\
\hline C & -4.112360 & 0.586350 & -4.256360 \\
\hline $\mathrm{F}$ & -3.347020 & -0.440030 & -0.848330 \\
\hline $\mathrm{F}$ & -6.070020 & -0.410610 & -0.750940 \\
\hline $\mathrm{F}$ & -7.510440 & 0.275560 & -2.970500 \\
\hline $\mathrm{F}$ & -6.226770 & 0.936020 & -5.295170 \\
\hline $\mathrm{F}$ & -3.509100 & 0.915780 & -5.402820 \\
\hline C & 7.177010 & 0.374710 & -1.633250 \\
\hline C & 8.571160 & 0.320060 & -1.475870 \\
\hline C & 9.376890 & 0.005020 & -2.583860 \\
\hline C & 8.785410 & -0.252970 & -3.832330 \\
\hline C & 7.387320 & -0.192880 & -3.961610 \\
\hline $\mathrm{F}$ & 6.430830 & 0.672780 & -0.556100 \\
\hline $\mathrm{F}$ & 9.134240 & 0.555790 & -0.287490 \\
\hline $\mathrm{F}$ & 10.702040 & -0.051800 & -2.448740 \\
\hline $\mathrm{F}$ & 9.552020 & -0.552630 & -4.882850 \\
\hline $\mathrm{F}$ & 6.850910 & -0.444630 & -5.159200 \\
\hline C & 2.043050 & 5.859220 & -4.290250 \\
\hline C & 2.054570 & 7.264570 & -4.307570 \\
\hline C & 1.687130 & 7.973170 & -3.151140 \\
\hline C & 1.310850 & 7.276760 & -1.990160 \\
\hline C & 1.304870 & 5.871710 & -1.998540 \\
\hline $\mathrm{F}$ & 2.397120 & 5.208640 & -5.403170 \\
\hline $\mathrm{F}$ & 2.406430 & 7.931070 & -5.409940 \\
\hline $\mathrm{F}$ & 1.695510 & 9.306750 & -3.155180 \\
\hline $\mathrm{F}$ & 0.967530 & 7.954620 & -0.891360 \\
\hline $\mathrm{F}$ & 0.943180 & 5.233030 & -0.879620 \\
\hline C & 2.974400 & 0.330650 & 2.447120 \\
\hline $\mathrm{H}$ & 2.656620 & 1.196550 & 3.064070 \\
\hline $\mathrm{H}$ & 2.882330 & -0.590520 & 3.058830 \\
\hline $\mathrm{H}$ & 4.029350 & 0.464710 & 2.134480 \\
\hline C & -1.667750 & -0.179660 & 7.260150 \\
\hline C & -5.726340 & -0.975330 & 4.007130 \\
\hline $\mathrm{O}$ & -4.647870 & -0.330510 & 6.616440 \\
\hline C & -4.996790 & 0.916080 & 7.207220 \\
\hline $\mathrm{H}$ & -6.082150 & 0.880780 & 7.445090 \\
\hline $\mathrm{H}$ & -4.441850 & 1.086080 & 8.158840 \\
\hline $\mathrm{H}$ & -4.805370 & 1.765570 & 6.514830 \\
\hline C & -0.217730 & -0.729590 & 7.201980 \\
\hline $\mathrm{H}$ & -0.182650 & -1.742420 & 6.744600 \\
\hline $\mathrm{H}$ & 0.472530 & -0.068380 & 6.636370 \\
\hline $\mathrm{H}$ & 0.185970 & -0.805140 & 8.235360 \\
\hline C & -2.408930 & -1.067080 & 8.290250 \\
\hline $\mathrm{H}$ & -1.842410 & -1.068550 & 9.247750 \\
\hline
\end{tabular}

\begin{tabular}{|c|c|c|c|}
\hline C & -4.104390 & 0.175420 & -3.482160 \\
\hline$C$ & 0.795710 & -4.831150 & -3.619820 \\
\hline C & 1.157140 & -5.567320 & -2.474860 \\
\hline C & 1.155870 & -6.972290 & -2.466470 \\
\hline C & 0.786370 & -7.669890 & -3.628920 \\
\hline C & 0.421460 & -6.962280 & -4.786750 \\
\hline C & 0.429070 & -5.556930 & -4.769690 \\
\hline $\mathrm{F}$ & 1.513480 & -4.927770 & -1.354620 \\
\hline $\mathrm{F}$ & 1.497780 & -7.648840 & -1.366630 \\
\hline $\mathrm{F}$ & 0.782490 & -9.003360 & -3.633170 \\
\hline $\mathrm{F}$ & 0.076340 & -7.629690 & -5.890630 \\
\hline $\mathrm{F}$ & 0.079030 & -4.907840 & -5.884930 \\
\hline C & -4.774730 & -0.160180 & -2.290270 \\
\hline C & -6.176450 & -0.153420 & -2.199650 \\
\hline C & -6.937670 & 0.196250 & -3.328240 \\
\hline C & -6.296170 & 0.535980 & -4.531620 \\
\hline C & -4.892390 & 0.521930 & -4.596080 \\
\hline $\mathrm{F}$ & -4.071320 & -0.492770 & -1.198910 \\
\hline $\mathrm{F}$ & -6.789470 & -0.469560 & -1.055600 \\
\hline $\mathrm{F}$ & -8.269130 & 0.207210 & -3.256410 \\
\hline $\mathrm{F}$ & -7.022910 & 0.865380 & -5.601810 \\
\hline $\mathrm{F}$ & -4.307990 & 0.848940 & -5.753320 \\
\hline C & 6.433110 & 0.335130 & -2.219210 \\
\hline C & 7.829100 & 0.286410 & -2.079160 \\
\hline C & 8.620730 & -0.034420 & -3.195690 \\
\hline C & 8.013190 & -0.303930 & -4.434020 \\
\hline C & 6.613150 & -0.249480 & -4.545460 \\
\hline $\mathrm{F}$ & 5.699750 & 0.639200 & -1.133500 \\
\hline $\mathrm{F}$ & 8.406860 & 0.533330 & -0.900260 \\
\hline $\mathrm{F}$ & 9.947610 & -0.085860 & -3.078250 \\
\hline $\mathrm{F}$ & 8.766930 & -0.609210 & -5.491950 \\
\hline $\mathrm{F}$ & 6.061560 & -0.513120 & -5.733450 \\
\hline C & 1.264530 & 5.793250 & -4.807590 \\
\hline C & 1.273330 & 7.198340 & -4.838360 \\
\hline C & 0.910320 & 7.917260 & -3.686920 \\
\hline C & 0.541800 & 7.231010 & -2.517420 \\
\hline C & 0.539610 & 5.826010 & -2.511950 \\
\hline $\mathrm{F}$ & 1.612730 & 5.133240 & -5.916910 \\
\hline $\mathrm{F}$ & 1.618000 & 7.854820 & -5.949010 \\
\hline $\mathrm{F}$ & 0.915530 & 9.250660 & -3.703780 \\
\hline $\mathrm{F}$ & 0.202390 & 7.918230 & -1.423450 \\
\hline $\mathrm{F}$ & 0.186120 & 5.197970 & -1.384420 \\
\hline $\mathrm{H}$ & 3.748130 & 0.347260 & 1.285720 \\
\hline $\mathrm{H}$ & 2.732990 & -0.650340 & 2.416310 \\
\hline $\mathrm{H}$ & 2.577480 & 1.133320 & 2.433900 \\
\hline C & -4.310450 & -0.816480 & 5.270560 \\
\hline C & 0.103710 & -0.087730 & 8.039060 \\
\hline 0 & -2.937720 & -0.224430 & 7.743540 \\
\hline C & -3.197770 & 1.008780 & 8.403600 \\
\hline $\mathrm{H}$ & -4.250850 & 0.982080 & 8.759330 \\
\hline $\mathrm{H}$ & -2.538730 & 1.142820 & 9.292010 \\
\hline $\mathrm{H}$ & -3.068920 & 1.875440 & 7.718200 \\
\hline C & -4.719760 & -1.152940 & 3.818580 \\
\hline $\mathrm{H}$ & -4.614410 & -0.282990 & 3.134420 \\
\hline $\mathrm{H}$ & -4.129110 & -1.997200 & 3.401240 \\
\hline $\mathrm{H}$ & -5.789560 & -1.455670 & 3.805000 \\
\hline C & -4.608160 & -2.071150 & 6.130410 \\
\hline $\mathrm{H}$ & -4.363370 & -1.902780 & 7.198200 \\
\hline
\end{tabular}




$\begin{array}{lrrrrrrr}\mathrm{H} & -3.441150 & -0.735040 & 8.504120 & \mathrm{H} & -5.686860 & -2.335660 & 6.053350 \\ \mathrm{H} & -2.469000 & -2.117600 & 7.930040 & \mathrm{H} & -4.018760 & -2.942740 & 5.768400 \\ \mathrm{C} & -1.570160 & 1.284850 & 7.759630 & \mathrm{C} & -5.202310 & 0.365670 & 5.723920 \\ \mathrm{H} & -0.968850 & 1.898300 & 7.052810 & \mathrm{H} & -5.101610 & 0.583180 & 6.804380 \\ \mathrm{H} & -2.560550 & 1.772200 & 7.863060 & \mathrm{H} & -4.959510 & 1.289750 & 5.153610 \\ \mathrm{H} & -1.069540 & 1.318430 & 8.753750 & \mathrm{H} & -6.272400 & 0.123730 & 5.533840 \\ \mathrm{C} & -6.669290 & 0.215900 & 4.306050 & \mathrm{C} & 1.535720 & -0.635990 & 7.798480 \\ \mathrm{H} & -6.694750 & 0.478000 & 5.381200 & \mathrm{H} & 1.515660 & -1.640580 & 7.322060 \\ \mathrm{H} & -6.364360 & 1.118340 & 3.731040 & \mathrm{H} & 2.155740 & 0.036490 & 7.168350 \\ \mathrm{H} & -7.709050 & -0.042430 & 4.002950 & \mathrm{H} & 2.058490 & -0.730320 & 8.775240 \\ \mathrm{C} & -6.128140 & -2.203150 & 4.863170 & \mathrm{C} & -0.509990 & -0.994990 & 9.133560 \\ \mathrm{H} & -6.021590 & -2.000500 & 5.947470 & \mathrm{H} & 0.160010 & -1.003750 & 10.021780 \\ \mathrm{H} & -7.187610 & -2.476120 & 4.657200 & \mathrm{H} & -1.514700 & -0.676230 & 9.465550 \\ \mathrm{H} & -5.495520 & -3.082110 & 4.607820 & \mathrm{H} & -0.601040 & -2.041110 & 8.767110 \\ \mathrm{C} & -5.948000 & -1.362820 & 2.527790 & \mathrm{C} & 0.263440 & 1.367520 & 8.549340 \\ \mathrm{H} & -5.764980 & -0.519530 & 1.828500 & \mathrm{H} & 0.775020 & 1.994590 & 7.785930 \\ \mathrm{H} & -5.302730 & -2.213670 & 2.218090 & \mathrm{H} & -0.706630 & 1.851050 & 8.781570 \\ \mathrm{H} & -7.005190 & -1.679350 & 2.389760 & \mathrm{H} & 0.881190 & 1.384490 & 9.475590\end{array}$




\section{Experimental Section}

\section{II.1 Materials and Methods}

Chromatography stationary phases: Column chromatography was carried out using $0.04-0.063 \mathrm{~mm}$ mesh silica gel. $R_{\mathrm{f}}$ values were determined by thin layer chromatography on Polygram ${ }^{\circledR} \mathrm{Sil} G / \mathrm{UV}_{254}$ (0.2 $\mathrm{mm}$ particle size).

NMR spectroscopy: NMR spectra were measured in deuterated solvents. Chemical shifts are either calibrated to the internal standard TMS or using residual protonated solvent signals $\left({ }^{1} \mathrm{H}: \delta\left(\mathrm{CHCl}_{3}\right)=\right.$ $7.26 \mathrm{ppm}, \delta\left(\mathrm{CD}_{3} \mathrm{CN}\right)=1.94 \mathrm{ppm}, \delta$ (toluene-d8) $=2.08 \mathrm{ppm} ;{ }^{13} \mathrm{C}: \delta\left(\mathrm{CHCl}_{3}\right)=77.16 \mathrm{ppm}, \delta\left(\mathrm{CD}_{3} \mathrm{CN}\right)=$ $1.32 \mathrm{ppm}, \delta$ (toluene-d8) $=137.48 \mathrm{ppm}$; deuteration grade $99.8 \%$ ). Reference for ${ }^{19} \mathrm{~F} \mathrm{NMR}$ spectra is $\mathrm{CCl}_{3} \mathrm{~F}$ to which the spectrometer frequency is calibrated. Assignments of ${ }^{1} \mathrm{H}$ and ${ }^{13} \mathrm{C}$ nuclei were confirmed by the 2D methods COSY, HSQC and HMBC. The signal multiplicities are abbreviated as $\mathrm{s}$ (singlet), d (doublet), t (triplet), q (quartet), quint (quintet), $m$ (multiplet) and br (broad signal).

Mass spectrometry: The high resolution (HR) mass spectra were measured on a time-of-flight (TOF) mass spectrometer with electron ionization (EI).

IR spectroscopy: Infrared spectra were recorded on a FT-IR spectrometer with an ATR unit. Signal intensities were abbreviated with w (weak), $\mathrm{m}$ (medium), $\mathrm{s}$ (strong) and vs (very strong). Broad signals are additionally labeled with br.

UV-vis spectroscopy: UV-visible absorption spectra were recorded on a two-beam spectrophotometer with a thermostat $\left(T=20^{\circ} \mathrm{C}\right)$. Measurements were performed in quartz cells of $1 \mathrm{~cm}$ optical path length.

Light sources: Irradiation experiments were performed using LED light sources. 


\section{II.2 NMR Spectroscopy}
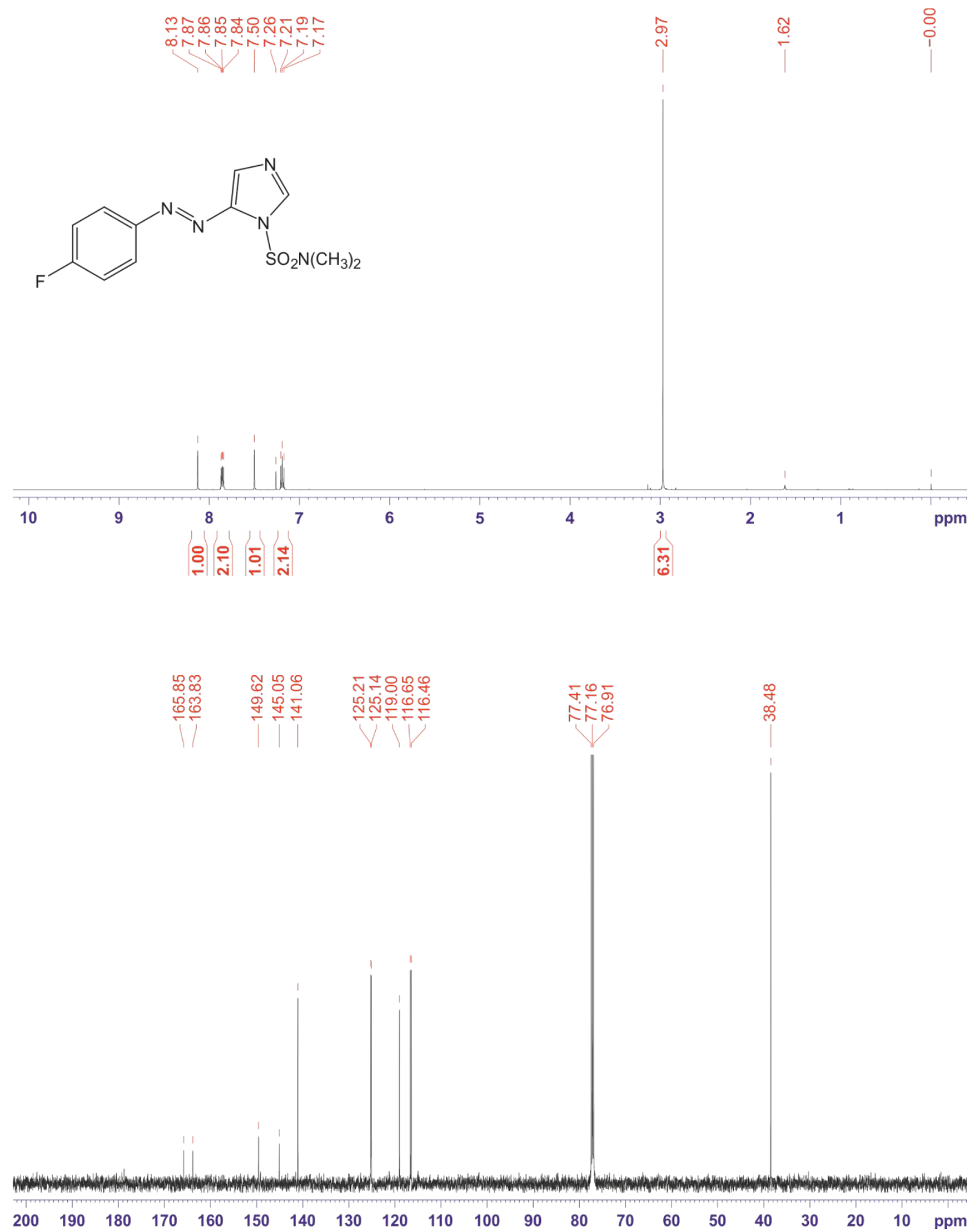

Figure S6. ${ }^{1} \mathrm{H}$ NMR spectrum (top) and ${ }^{13} \mathrm{C}$ NMR spectrum (bottom) of 1-( $N, N$-Dimethylsulfamoyl)-5(4'-fluorophenylazo)imidazole. Spectra were measured in chloroform- $d_{1}$ at $300 \mathrm{~K}$. 

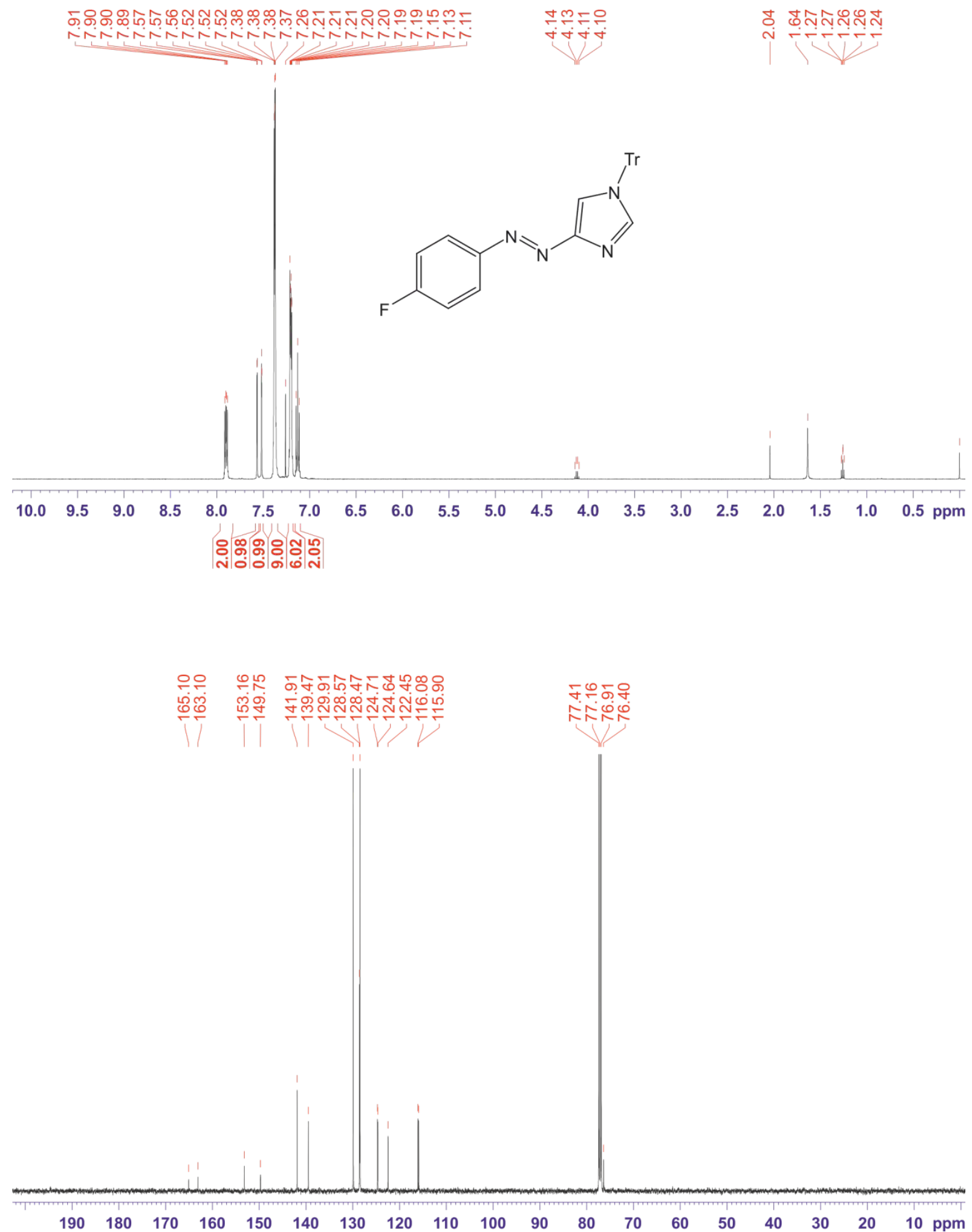

Figure S7. ${ }^{1} \mathrm{H}$ NMR spectrum (top) and ${ }^{13} \mathrm{C}$ NMR spectrum (bottom) of 1-Trityl-4-(4'fluorophenylazo)imidazole. Spectra were measured in chloroform- $d_{1}$ at $300 \mathrm{~K}$. 


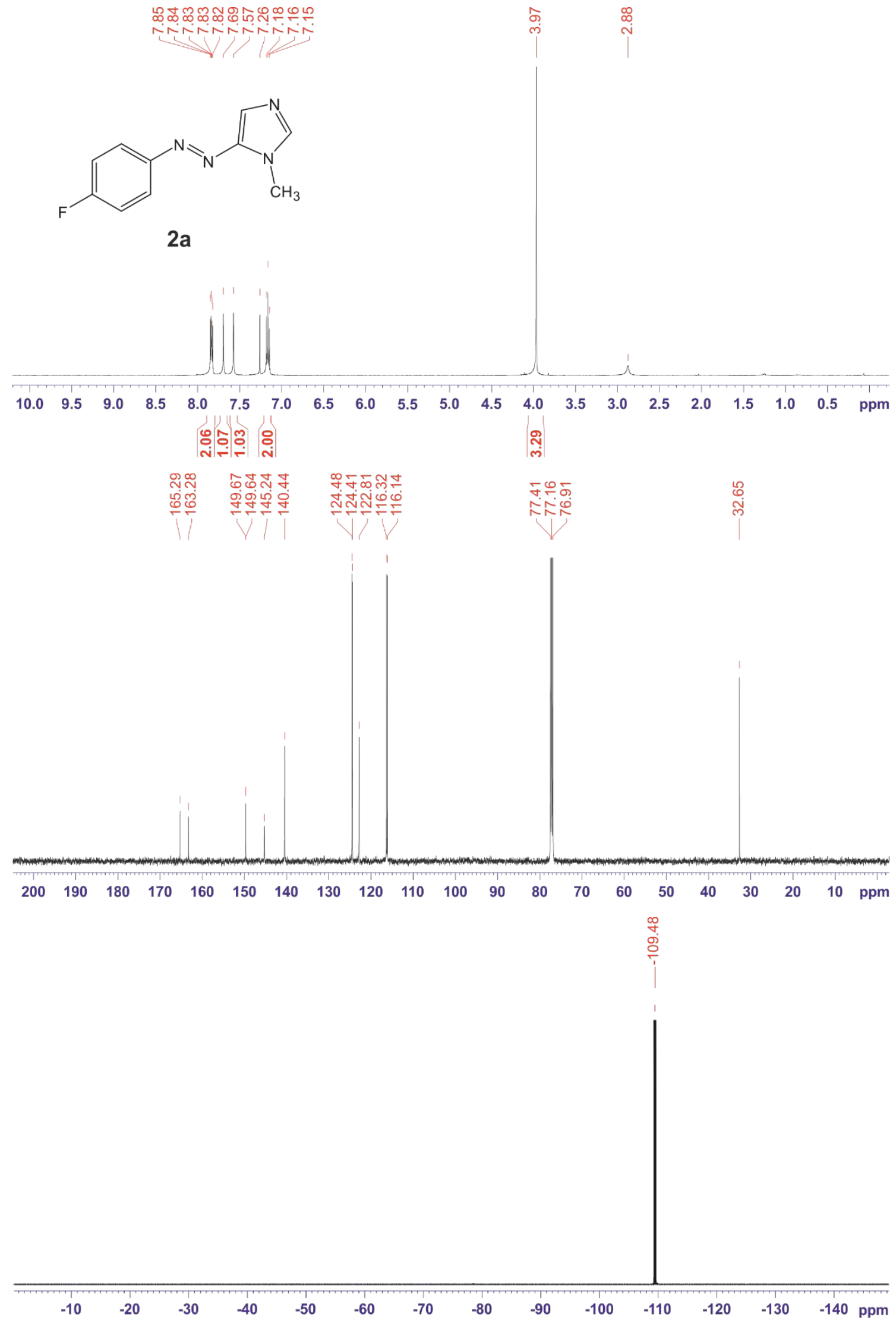

Figure S8. ${ }^{1} \mathrm{H}$ NMR spectrum (top), ${ }^{13} \mathrm{C}$ NMR spectrum (middle) and ${ }^{19} \mathrm{~F}$ NMR spectrum (bottom) of 1Methyl-5-(4'-fluorophenylazo)imidazole (2a). Spectra were measured in chloroform- $d_{1}$ at $300 \mathrm{~K}$. 

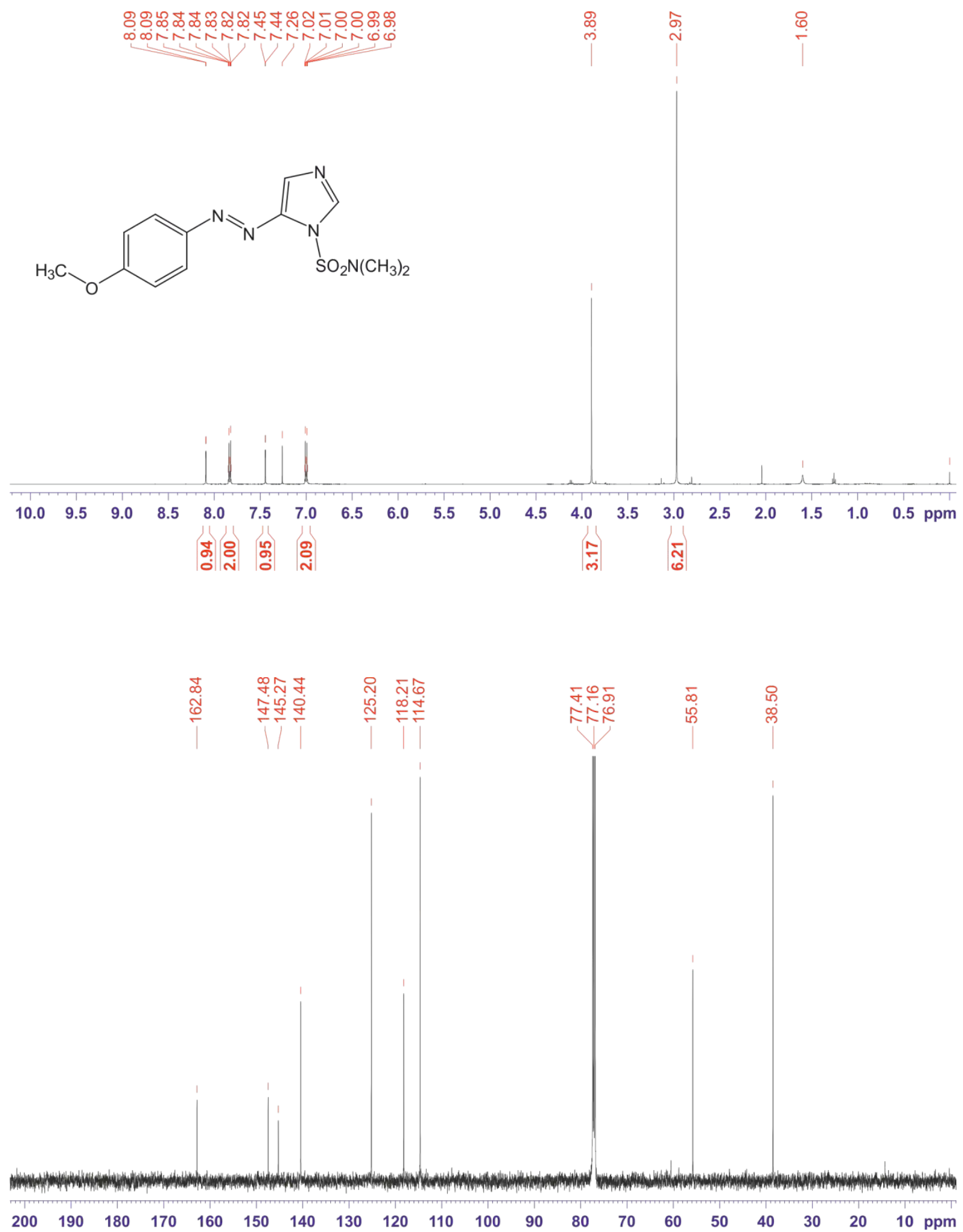

Figure S9. ${ }^{1} \mathrm{H}$ NMR spectrum (top) and ${ }^{13} \mathrm{C}$ NMR spectrum (bottom) of 1-(N,N-Dimethylsulfamoyl)-5(4'-methoxyphenylazo)imidazole. Spectra were measured in chloroform- $\mathrm{d}_{1}$ at $300 \mathrm{~K}$. 

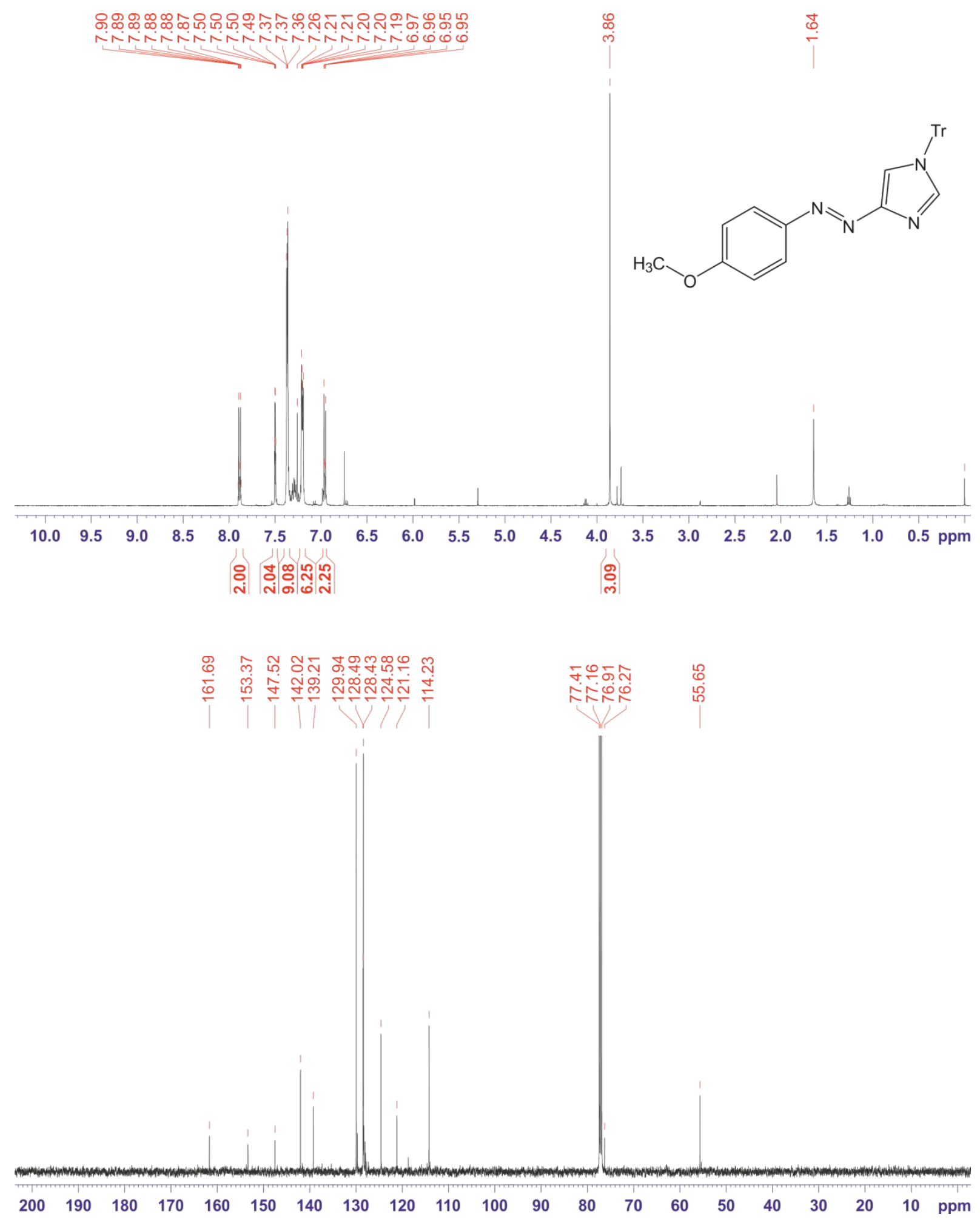

Figure S10. ${ }^{1} \mathrm{H}$ NMR spectrum (top) and ${ }^{13} \mathrm{C}$ NMR spectrum (bottom) of 1-Trityl-4-(4'methoxyphenylazo)imidazole. Spectra were measured in chloroform- $\mathrm{d}_{1}$ at $300 \mathrm{~K}$. 


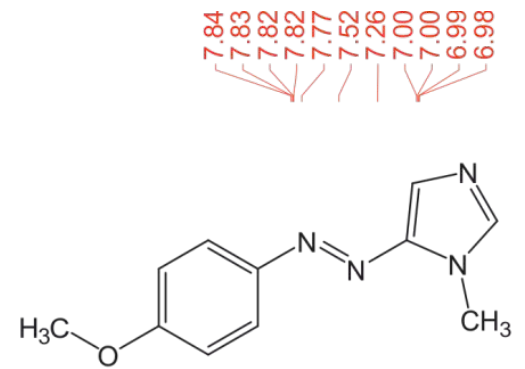

2b
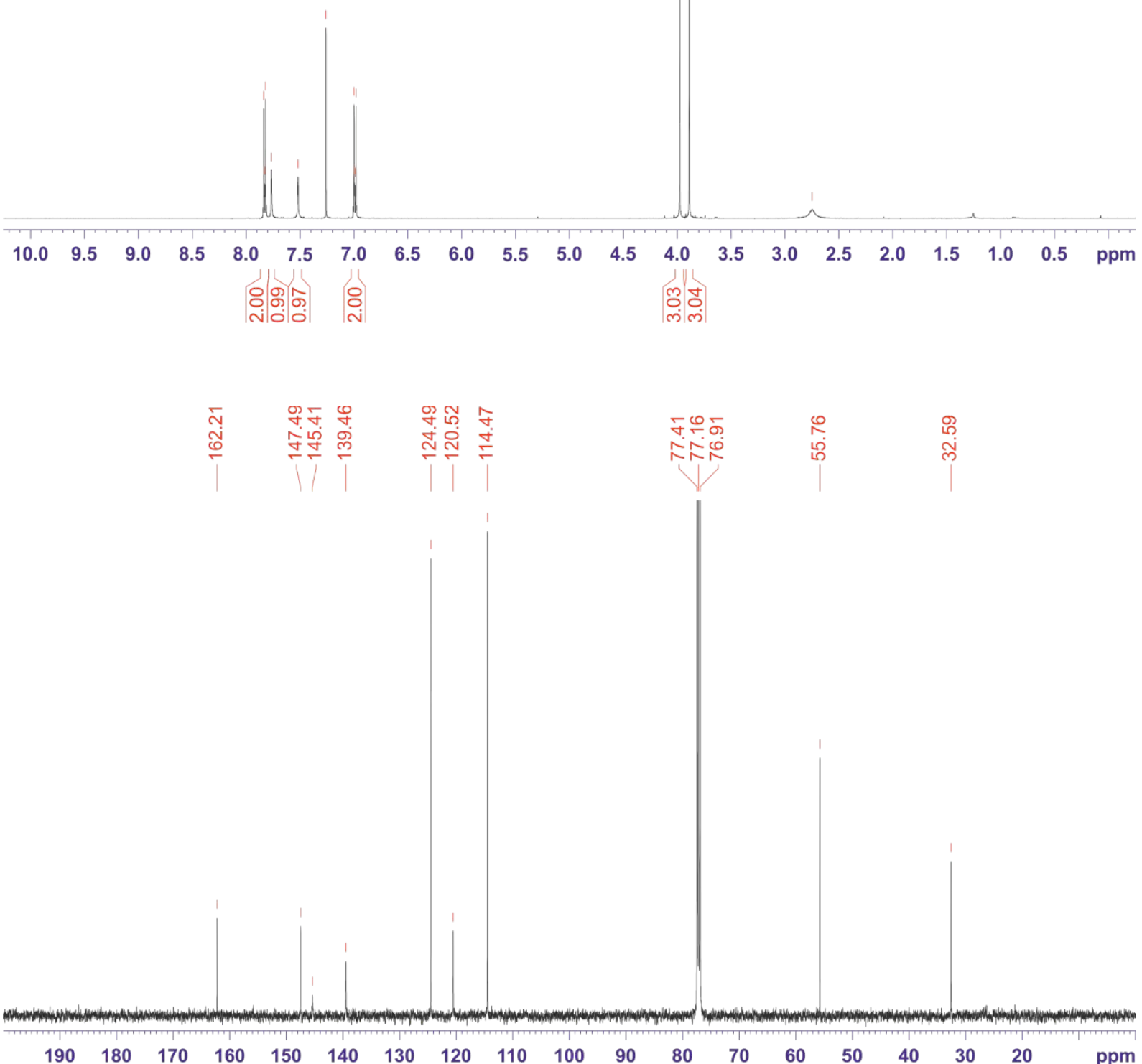

Figure S11. ${ }^{1} \mathrm{H}$ NMR spectrum (top) and ${ }^{13} \mathrm{C}$ NMR spectrum (bottom) of 1-Methyl-5-(4'methoxyphenylazo)imidazole (2b). Spectra were measured in chloroform- $\mathrm{d}_{1}$ at $300 \mathrm{~K}$. 

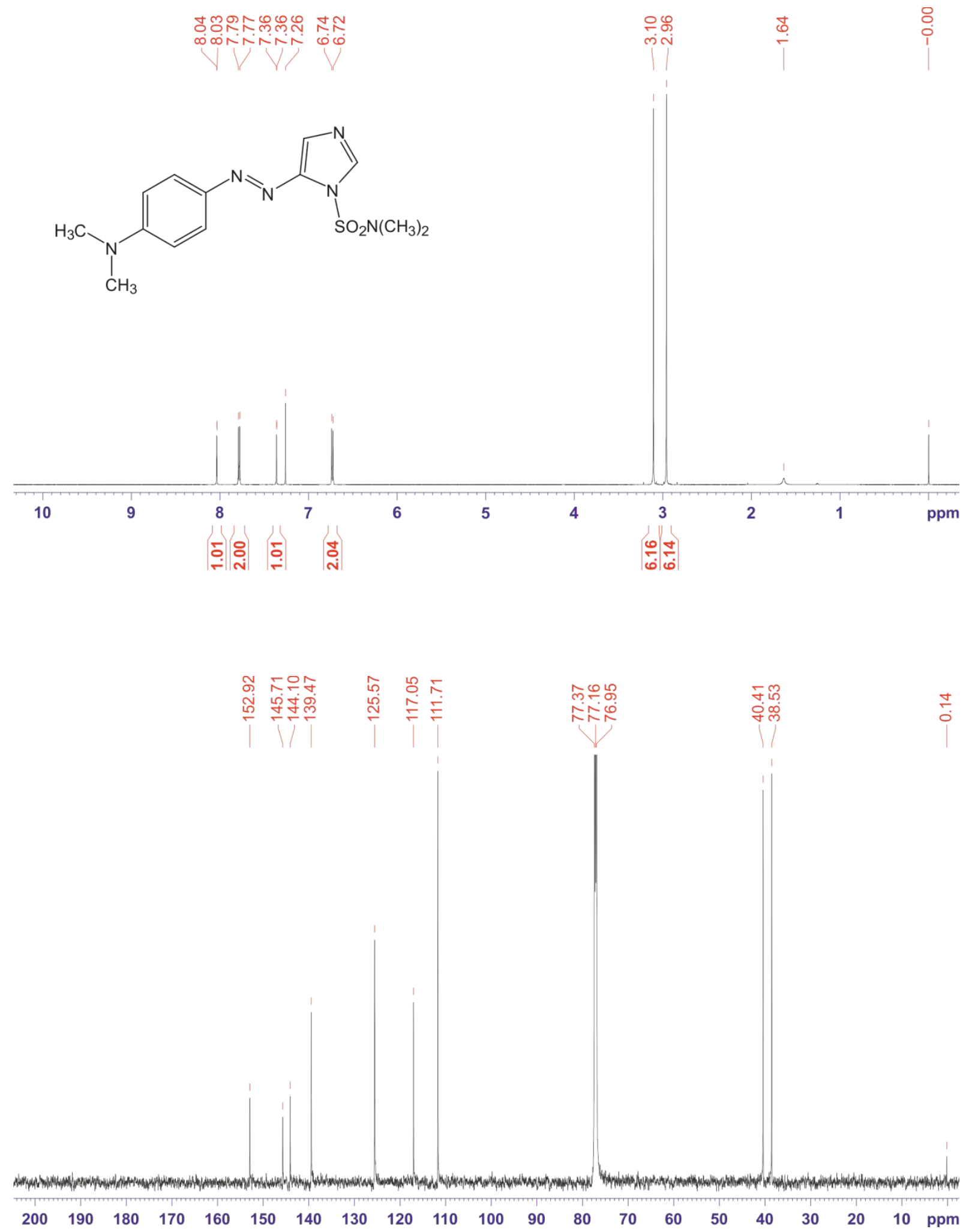

Figure S12. ${ }^{1} \mathrm{H}$ NMR spectrum (top) and ${ }^{13} \mathrm{C}$ NMR spectrum (bottom) of 1-( $N, N$-Dimethylsulfamoyl)-5$\left(4^{\prime}-\mathrm{N}, \mathrm{N}\right.$-dimethylaminophenylazo)imidazole. Spectra were measured in chloroform- $\mathrm{d}_{1}$ at $300 \mathrm{~K}$. 

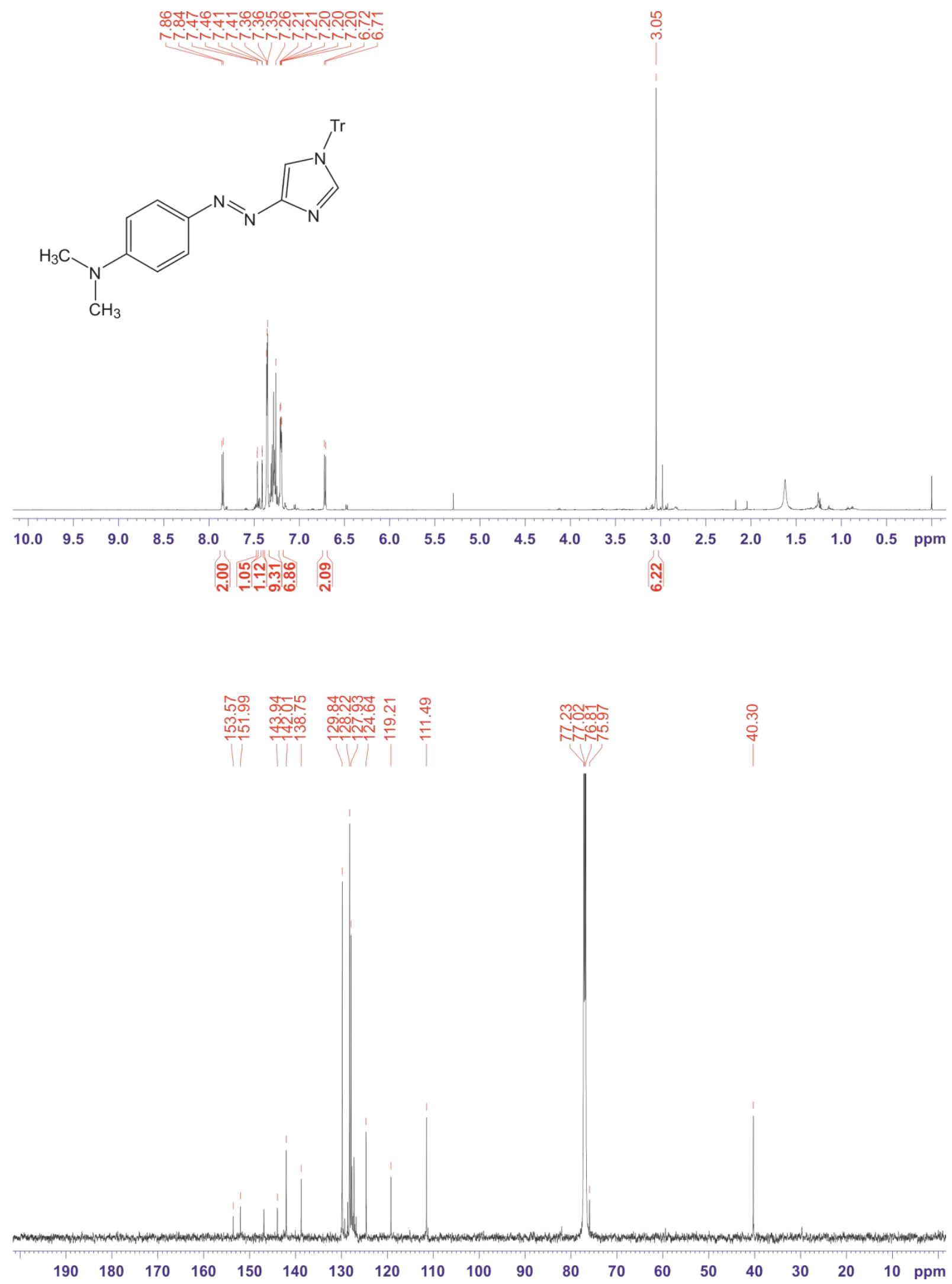

Figure S13. ${ }^{1} \mathrm{H}$ NMR spectrum (top) and ${ }^{13} \mathrm{C}$ NMR spectrum (bottom) of 1-Trityl-4-(4'-N,Ndimethylaminophenylazo)imidazole. Spectra were measured in chloroform- $d_{1}$ at $300 \mathrm{~K}$. 


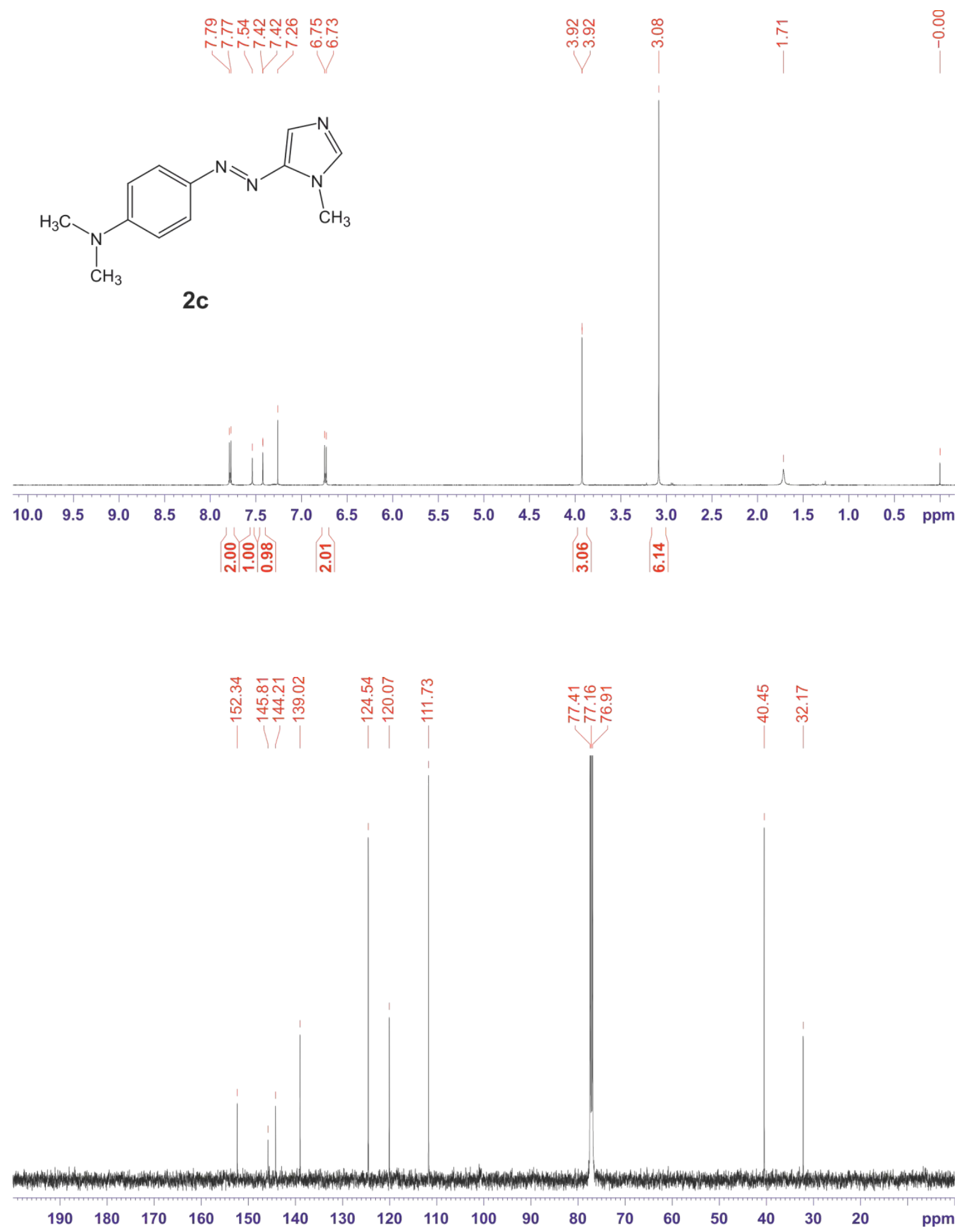

Figure S14. ${ }^{1} \mathrm{H}$ NMR spectrum (top) and ${ }^{13} \mathrm{C}$ NMR spectrum (bottom) of 1-Methyl-5-(4'-N,Ndimethylaminophenylazo)imidazole (2c). Spectra were measured in chloroform- $\mathrm{d}_{1}$ at $300 \mathrm{~K}$. 


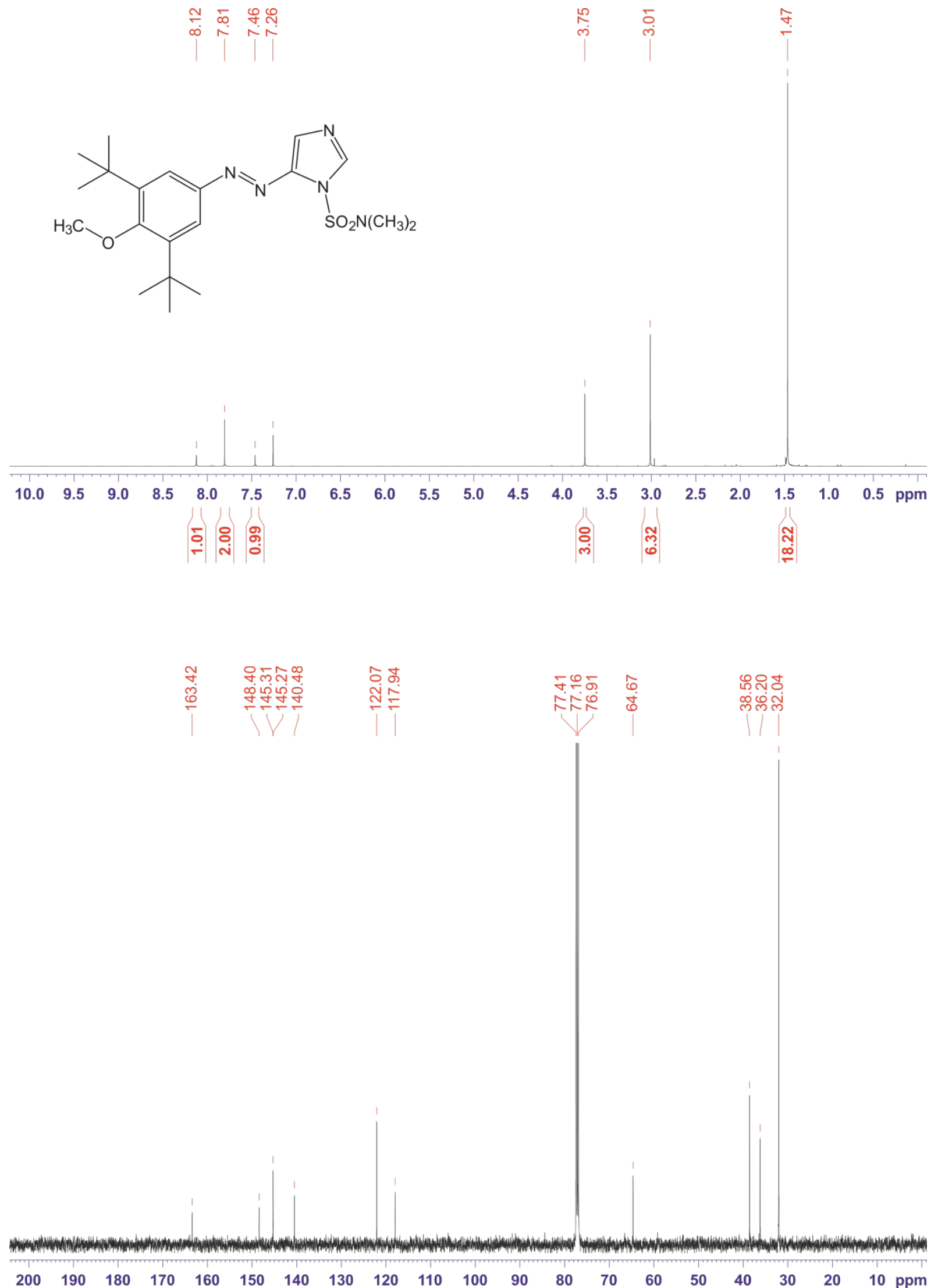

Figure S15. ${ }^{1} \mathrm{H}$ NMR spectrum (top) and ${ }^{13} \mathrm{C}$ NMR spectrum (bottom) of 1-(N,N-Dimethylsulfamoyl)-5$\left(3^{\prime}, 5^{\prime}\right.$-di-tert-butyl-4'-methoxyphenylazo)imidazole. Spectra were measured in chloroform- $d_{3}$ at $300 \mathrm{~K}$. 


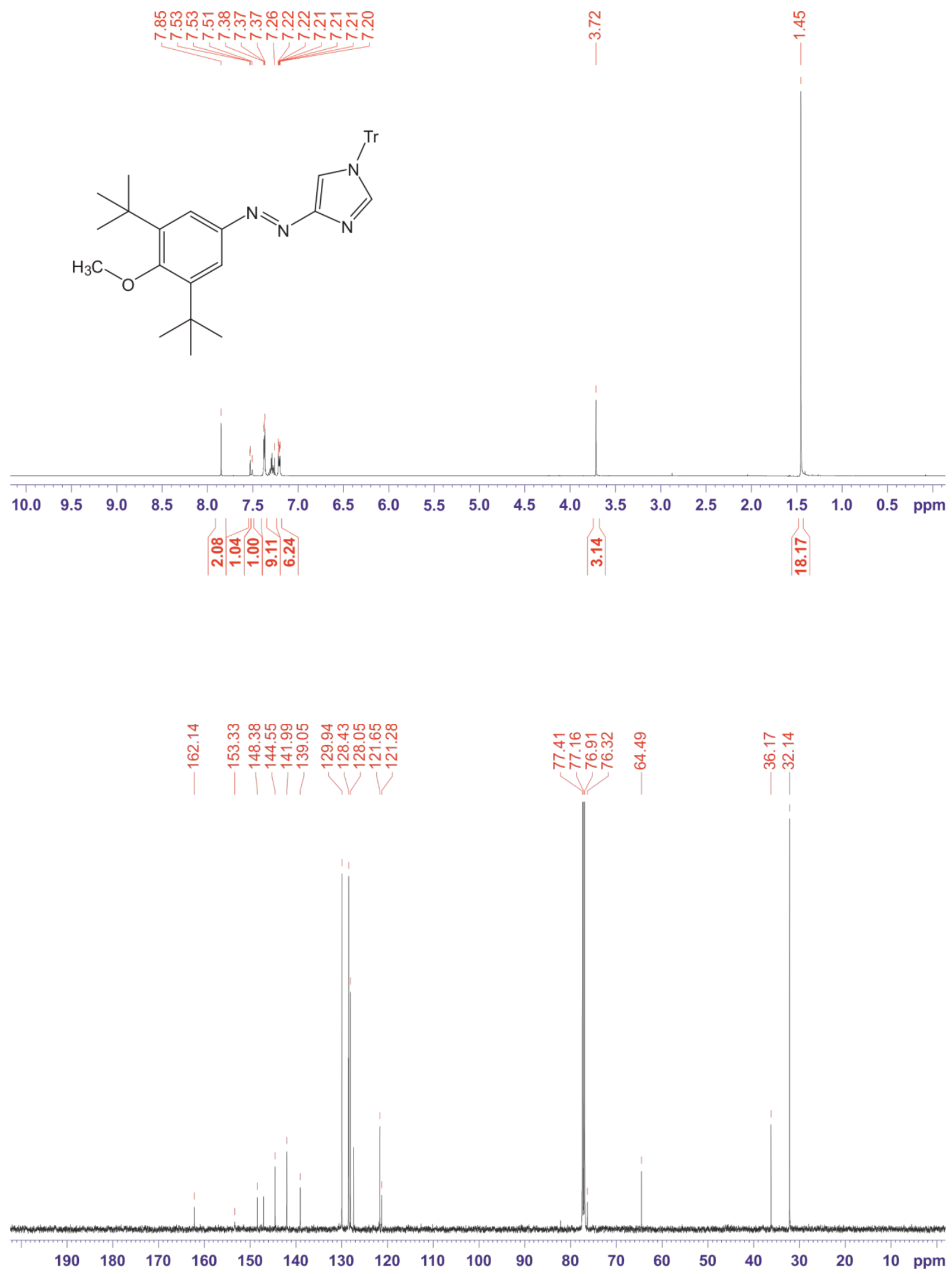

Figure S16. ${ }^{1} \mathrm{H}$ NMR spectrum (top) and ${ }^{13} \mathrm{C}$ NMR spectrum (bottom) of 1-Trityl-4-(3',5'-di-tert-butyl$4^{\prime}$-methoxyphenylazo)imidazole. Spectra were measured in chloroform- $d_{3}$ at $300 \mathrm{~K}$. 

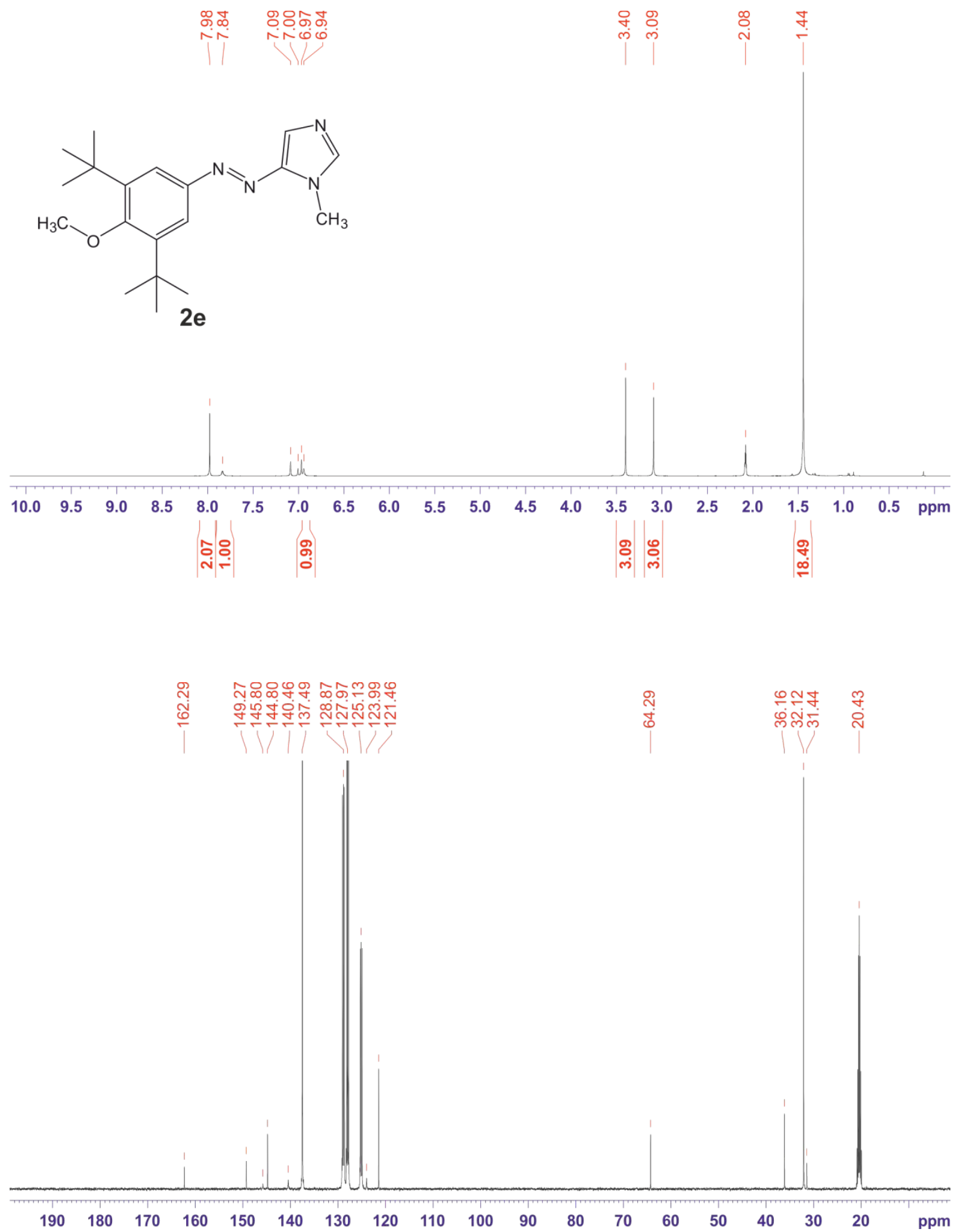

Figure S17. ${ }^{1} \mathrm{H}$ NMR spectrum (top) and ${ }^{13} \mathrm{C}$ NMR spectrum (bottom) of 1-Methyl-5-(3',5'-di-tertbutyl-4'-methoxyphenylazo)imidazole (2e). Spectra were measured in toluene- $\mathrm{d}_{8}$ at $300 \mathrm{~K}$. 


\section{II.3 UV-vis Spectroscopy}
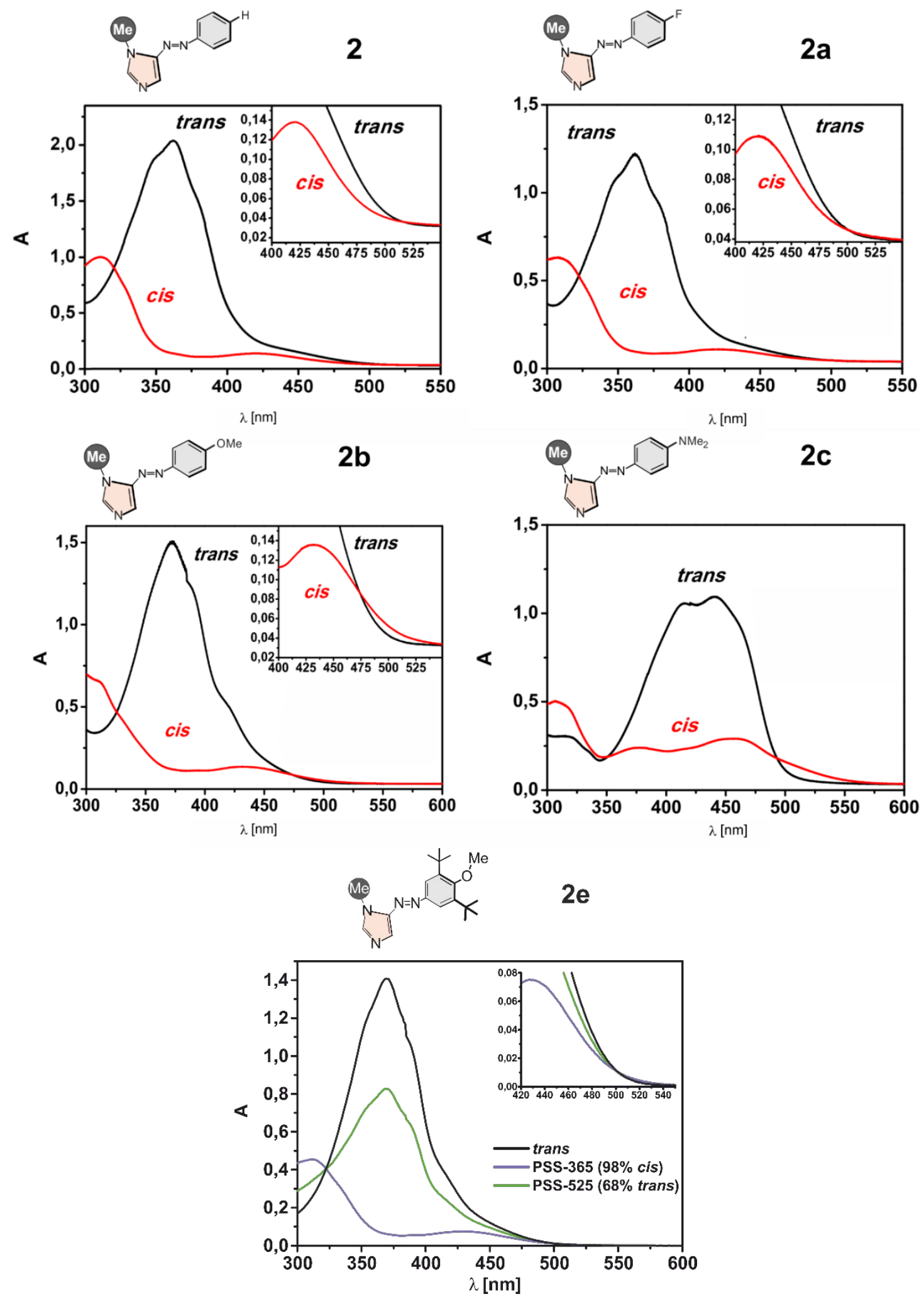

Figure S18. UV-vis spectra of the parent system 2 (top left) and its corresponding derivatives 2a (top right), $\mathbf{2 b}$ (middle left), $\mathbf{2 c}$ (middle right). The spectrum of the cis isomer is presented in red and the spectrum of the trans isomer is shown in black. UV-vis spectra of azoimidazole $\mathbf{2 e}$ (bottom). The spectrum of pure trans isomer (black line) was measured after storage in the dark for 2 days at $40{ }^{\circ} \mathrm{C}$. The spectra of PSS-365 (violet line) and PSS-525 (green line) were measured after irradiation for 15 minutes with the respective wavelength. 


\section{II.4 Thermal reisomerization of Phenylazoimidazoles $\mathbf{2 b}$ and $\mathbf{2 e}$}

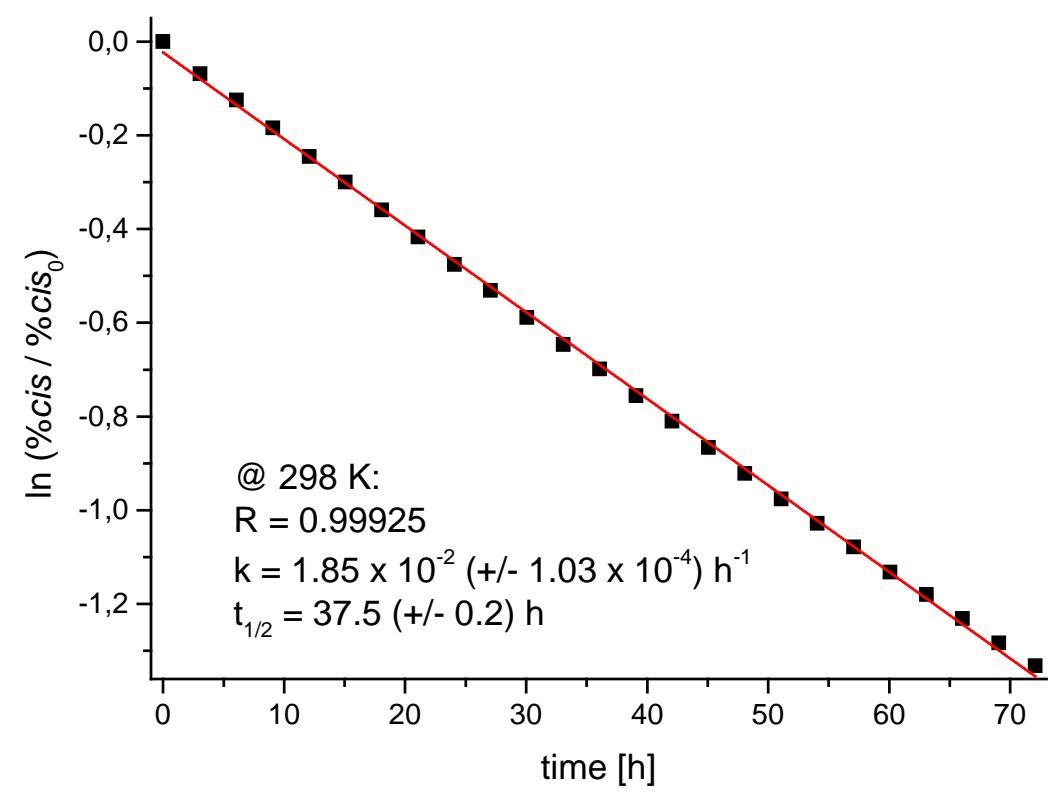

Figure S19. First order kinetics plot for the thermal cis to trans isomerization of 1-methyl-5(4'-methoxyphenylazo)imidazole $\mathbf{2} \mathbf{b}$ in toluene- $\mathrm{d}_{8}$. Cis ratios were determined by ${ }^{1} \mathrm{H}$ NMR spectroscopy.

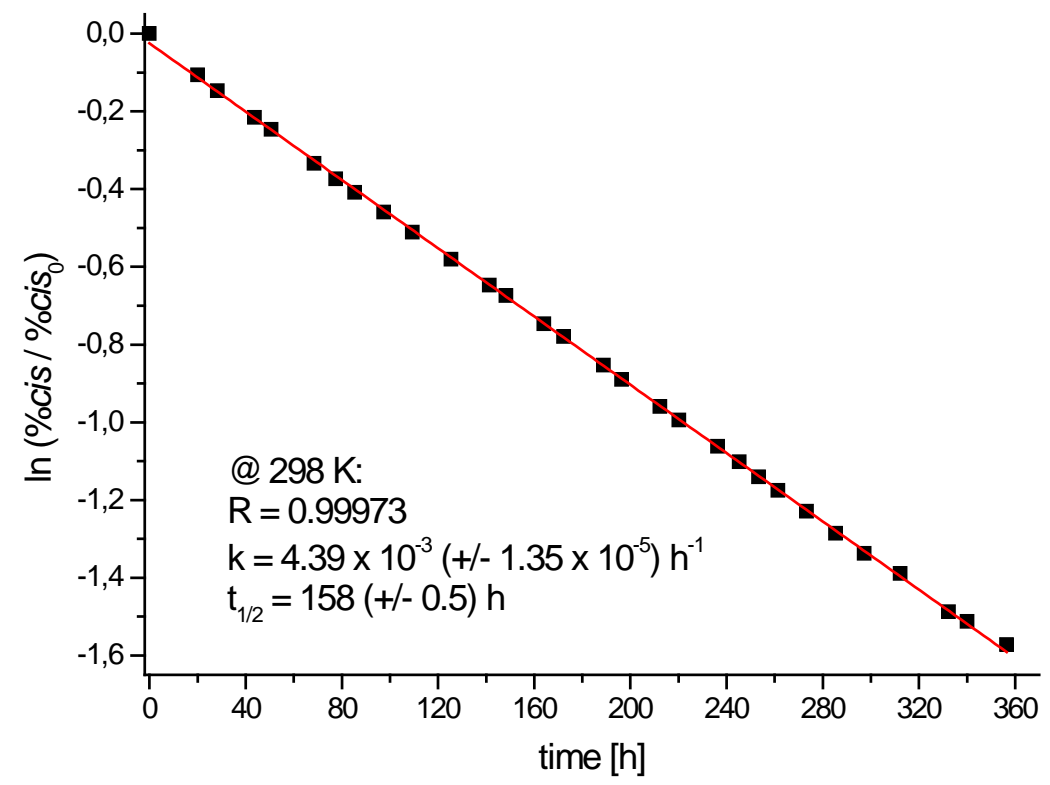

Figure S20. First order kinetics plot for the thermal cis to trans isomerization of 1-methyl-5- $\left(3^{\prime}, 5^{\prime}\right.$-ditert-butyl-4'-methoxyp-henylazo)imidazole $\mathbf{2 e}$ in toluene- $\mathrm{d}_{8}$. Cis ratios were determined by ${ }^{1} \mathrm{H}$ NMR spectroscopy. 


\section{II.5 Association Constants and Thermodynamic Parameters ${ }^{4,5}$}

As we have described earlier, ${ }^{4}$ the paramagnetism of the $\mathrm{Ni}^{2+}$ in the penta- and hexacoordinated complexes gives rise to a strong downfield shift and a broadening of the pyrrole proton resonance in porphyrins such as 5,10,15,20-tetrakis(pentafluorophenyl)-nickel(II)porphyrin (NiTPPF 20 ). Due to the very rapid coordination/decoordination process a time averaged signal of the dia- and paramagnetic species is observed in the ${ }^{1} \mathrm{H}$ NMR spectra which can be used to determine the ratio of dia- and paramagnetic Ni-porphyrins. The signal of the pyrrole protons was followed as a function of the axial ligand concentration at four different temperatures ( $298 \mathrm{~K}, 308 \mathrm{~K}, 318 \mathrm{~K}$ and $328 \mathrm{~K}$ ). $\mathrm{K}_{15}$ and $\mathrm{K}_{2}$ were determined from these data for each temperature using a non-linear curve fitting. Arrhenius plots of the obtained association constants gave the thermodynamic parameters $\Delta \mathrm{H}$ and $\Delta \mathrm{S}$ of complex formation. A different NMR tube was used for each ligand concentration. Each ${ }^{1} \mathrm{H}-\mathrm{NMR}$ tube was filled with $25 \mu \mathrm{L}$ (Experim. with 1-methylimidazole (1-mim)) or $100 \mu \mathrm{L}$ (Experim. with PDLs 2, 2b and 2e), respectively, of a $9.70 \mathrm{mM}$ NiTPPF 20 solution in toluene- $d_{8}$. Then different amounts of a solution of the ligands (1-mim: 122 mM; 2: 267 mM; 2b: 258 mM; 2e: $248 \mathrm{~mm}$ ) in toluene-d $\mathrm{d}_{8}$ were added to obtain ligand/porphyrin ratios between 2:1 and 100:1, and the tubes were filled up to $500 \mu \mathrm{L}$ with toluene- $\mathrm{d}_{8}$ and sealed. We measured NMR spectra of the PDL/porphyrin solutions (as described above) after storage in the dark at $\geq 40^{\circ} \mathrm{C}$ overnight (large excess of trans) and after irradiation for 20 min with 365 $\mathrm{nm}$ (PSS with large excess of cis) at four different temperatures ( $298 \mathrm{~K}, 308 \mathrm{~K}, 318 \mathrm{~K}$ and $328 \mathrm{~K})$. NMR spectra of the $\mathrm{N}$-methylimidazole/porphyrin solutions were measured at four different temperatures $(298 \mathrm{~K}, 308 \mathrm{~K}, 318 \mathrm{~K}$ and $328 \mathrm{~K})$. The data from NMR titration were fitted to a model including all conceivable 1:1 and 1:2 complexes with a non-linear treatment using Excel Speciation Tool. ${ }^{5}$

Table S5: Summary of temperature dependend association constants of 1-methylimidazole and azoimidazoles $2,2 b$ and $2 e$ with NiTPPF $_{20}$ in toluene- $d_{8}$ obtained from non-linear fitting.

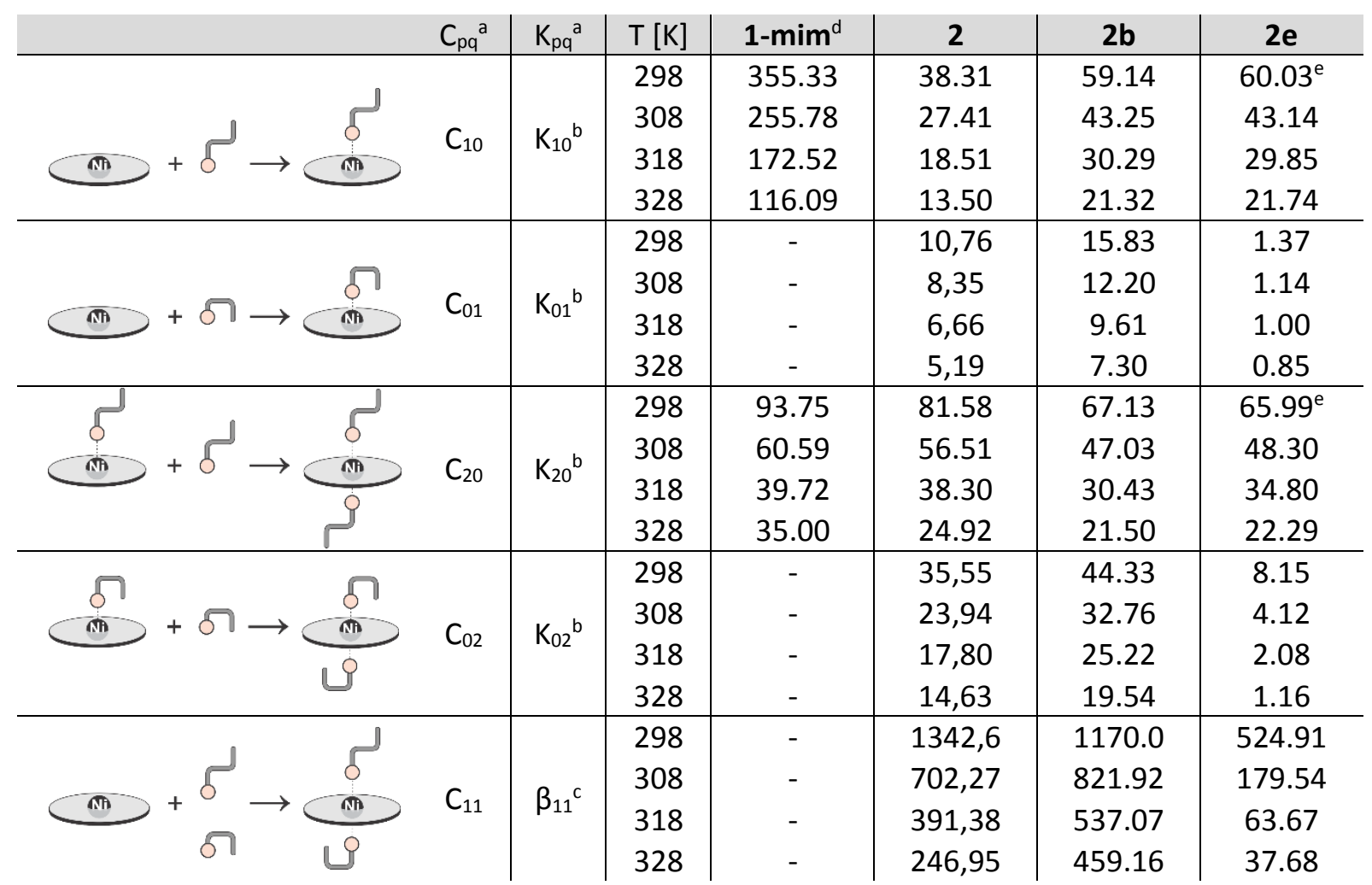

${ }^{a} C_{p q}$ represents the complex composition ( $p$ : PDL in trans configuration, q: PDL in cis configuration), $\mathrm{K}_{\mathrm{pq}}$ represents the corresponding association constant, ${ }^{\mathrm{b}}\left[\mathrm{L} \mathrm{mol}^{-1}\right] ;{ }^{\mathrm{c}}\left[\mathrm{L}^{2} \mathrm{~mol}^{-2}\right] ;{ }^{\mathrm{d}} \mathbf{1}$-mim obviously does not provide a trans and cis species. For the sake of simplicity the data for 1-mim were filed to the trans datasets of PDLs $\mathbf{2}, \mathbf{2} \mathbf{b} \& \mathbf{2 e}$; $^{\mathrm{e}} 300 \mathrm{~K}$. 


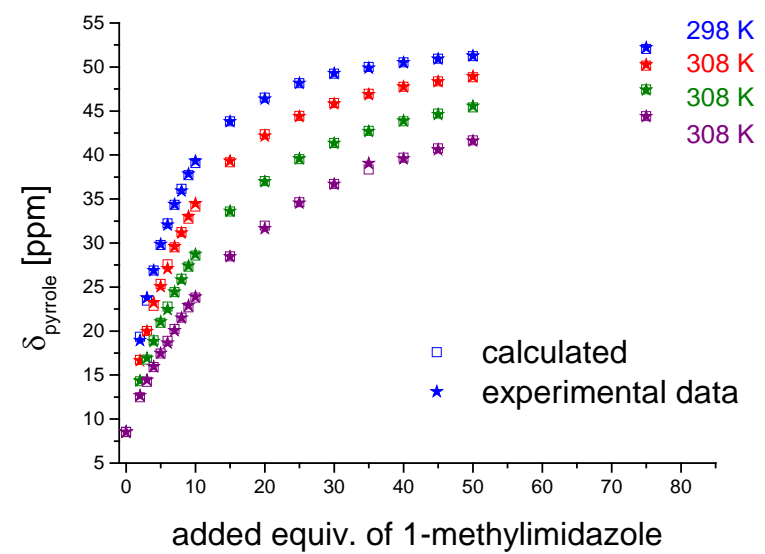

Figure S21: Fitting plots of measured and calculated pyrrole shifts of NiTPPF 20 upon coordination of 1methylimidazole.
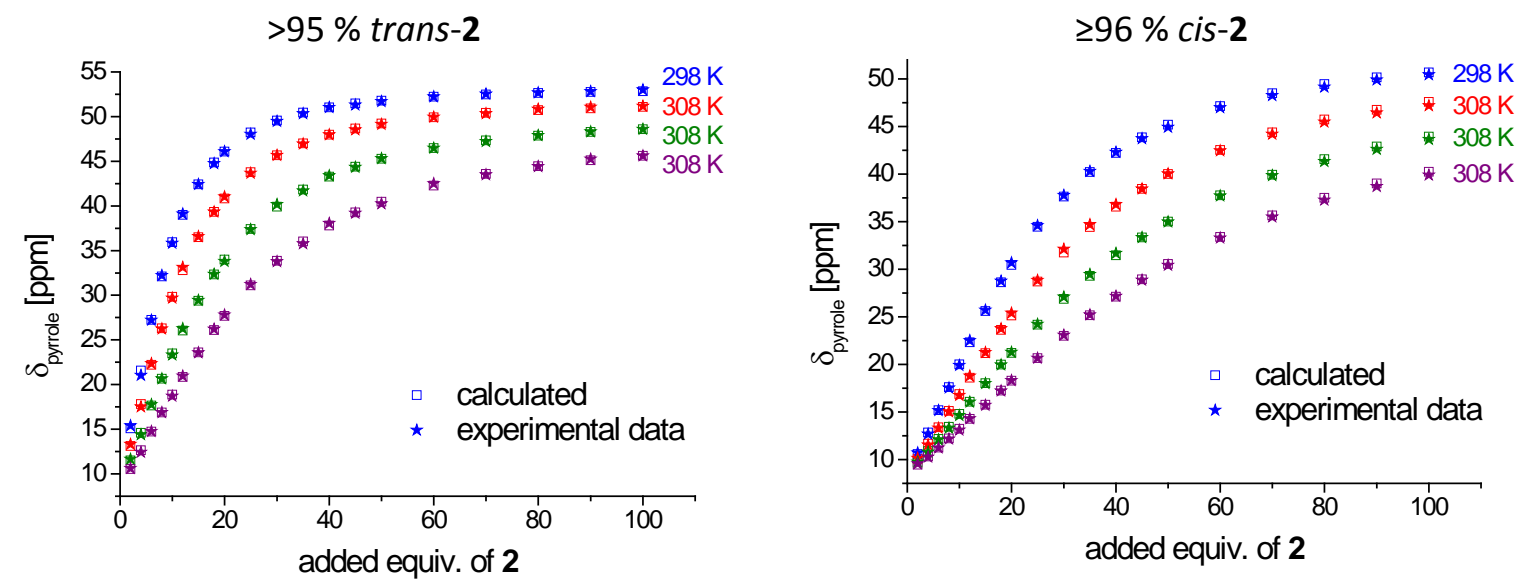

Figure S22: Fitting plots of measured and calculated pyrrole shifts of NiTPPF 20 upon coordination of trans (left) and cis (right) 1-methyl-5-phenylazoimidazole $\mathbf{2}$.

$\sim 100 \%$ trans-2b

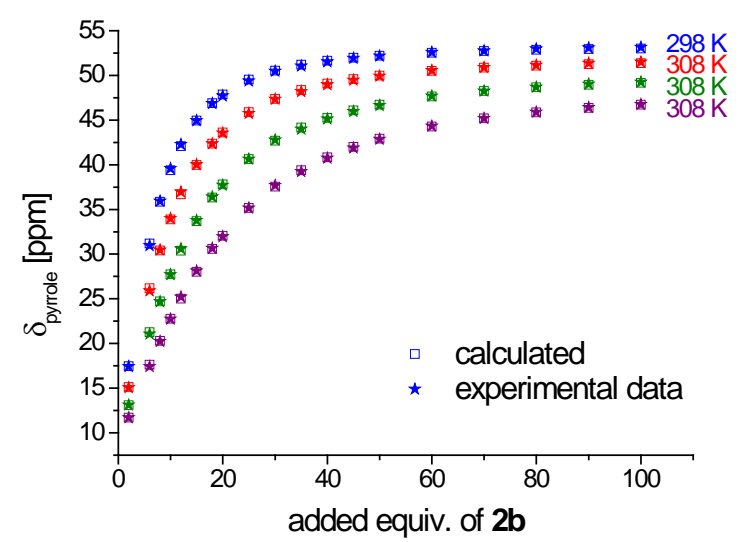

$\geq 80 \%$ cis- $\mathbf{2} \mathbf{b}$

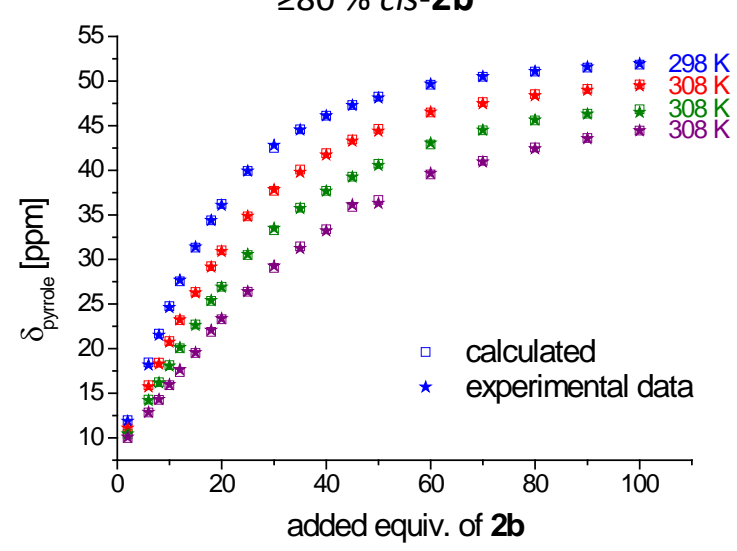

Figure S23: Fitting plots of measured and calculated pyrrole shifts of NiTPPF 20 upon coordination of trans (left) and cis (right) 1-methyl-5-(4'-methoxyphenylazo)imidazole $\mathbf{2} \mathbf{b}$. 

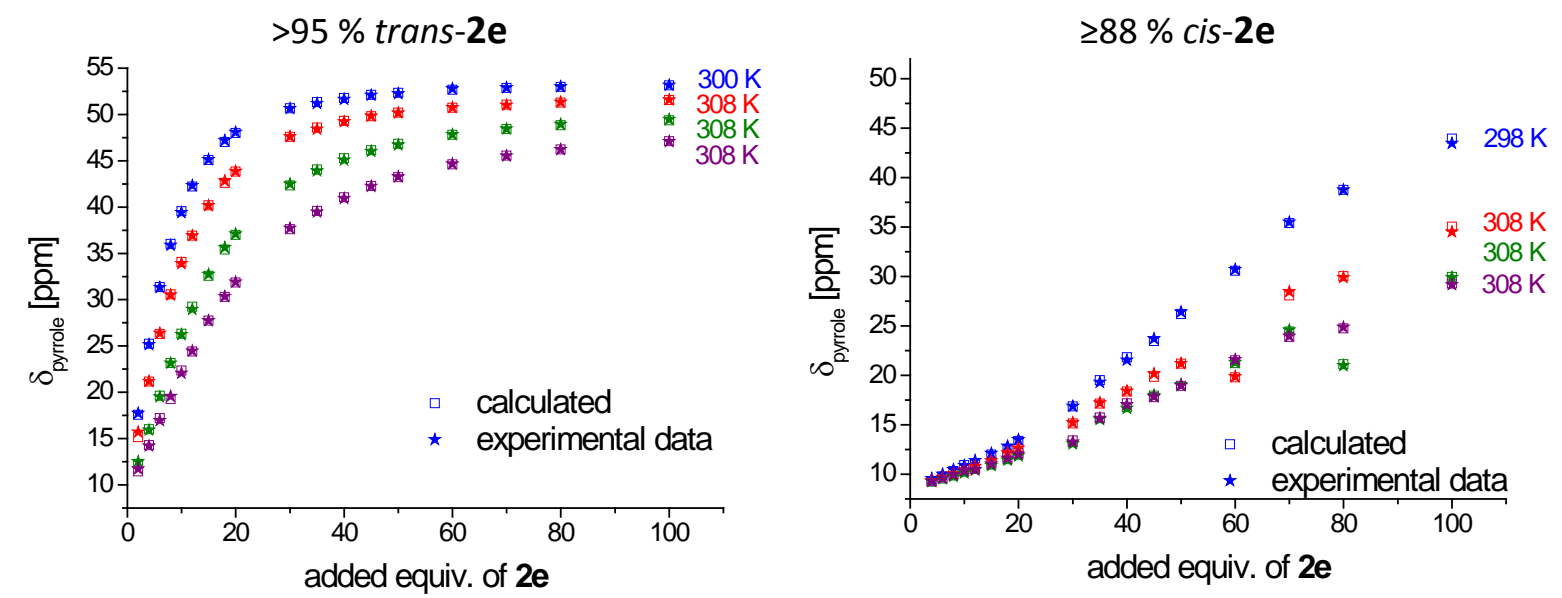

Figure S24: Fitting plots of measured and calculated pyrrole shifts of NiTPPF 20 upon coordination of trans (left) and cis (right) 1-methyl-5-(3',5'-di-tert-butyl-4'-methoxyphenylazo)imidazole 2e.

Table S6: Summary of thermodynamic binding parameters of 1-methylimidazole and azoimidazoles 2, $2 \mathrm{~b}$ and $2 \mathrm{e}$ with $\mathrm{Ni}(\mathrm{II}) \mathrm{TPPF}_{20}$ determined from Arrhenius plots of temperature dependent association constants.

\begin{tabular}{|c|c|c|c|c|c|c|c|c|c|}
\hline & & \multicolumn{2}{|c|}{$N-m^{2}{ }^{a}$} & \multicolumn{2}{|c|}{2} & \multicolumn{2}{|c|}{$2 b$} & \multicolumn{2}{|c|}{$2 e$} \\
\hline & & $\Delta \mathrm{H}_{\mathrm{f}}^{\mathrm{b}}$ & $\Delta S_{f}^{c}$ & $\Delta H_{f}^{b}$ & $\Delta S_{f^{c}}^{c}$ & $\Delta H_{f}^{b}$ & $\Delta S_{f}^{c}$ & $\Delta \mathrm{H}_{\mathrm{f}}^{\mathrm{b}}$ & $\Delta \mathrm{S}_{\mathrm{f}}{ }^{\mathrm{c}}$ \\
\hline \multirow{2}{*}{$\underset{+}{\tilde{D}}$} & $\mathrm{~K}_{1 \mathrm{~S}}$ & $\begin{array}{c}-7.27 \\
( \pm 0.37)\end{array}$ & $\begin{array}{c}-12.66 \\
( \pm 1.17)\end{array}$ & $\begin{array}{c}-6.84 \\
( \pm 0.20)\end{array}$ & $\begin{array}{c}-15.67 \\
( \pm 0.65)\end{array}$ & $\begin{array}{c}-6.63 \\
( \pm 0.26)\end{array}$ & $\begin{array}{c}-14.09 \\
( \pm 0.84)\end{array}$ & $\begin{array}{c}-7.10 \\
( \pm 0.15)\end{array}$ & $\begin{array}{c}-15.55 \\
( \pm 0.49)\end{array}$ \\
\hline & $\mathrm{K}_{2}$ & $\begin{array}{c}-6.59 \\
( \pm 0.91)\end{array}$ & $\begin{array}{c}-13.20 \\
( \pm 2.90)\end{array}$ & $\begin{array}{c}-7.65 \\
( \pm 0.35)\end{array}$ & $\begin{array}{c}-16.88 \\
( \pm 1.11)\end{array}$ & $\begin{array}{c}-7.48 \\
( \pm 0.27)\end{array}$ & $\begin{array}{c}-16.69 \\
( \pm 0.85)\end{array}$ & $\begin{array}{c}-7.45 \\
( \pm 0.57)\end{array}$ & $\begin{array}{c}-16,49 \\
( \pm 1.81)\end{array}$ \\
\hline \multirow{2}{*}{$\frac{n}{u}$} & $\mathrm{~K}_{1 \mathrm{~S}}$ & - & - & $\begin{array}{c}-4.67 \\
( \pm 0.11)\end{array}$ & $\begin{array}{c}-11.01 \\
( \pm 0.35)\end{array}$ & $\begin{array}{c}-4.97 \\
( \pm 0.16)\end{array}$ & $\begin{array}{c}-11.19 \\
( \pm 0.52)\end{array}$ & $\begin{array}{c}-3.01 \\
( \pm 0.11)\end{array}$ & $\begin{array}{c}-9.50 \\
( \pm 0.34)\end{array}$ \\
\hline & $\mathrm{K}_{2}$ & - & - & $\begin{array}{c}-5.77 \\
( \pm 0.49)\end{array}$ & $\begin{array}{c}-12.35 \\
( \pm 1.58)\end{array}$ & $\begin{array}{c}-5.28 \\
( \pm 0.06)\end{array}$ & $\begin{array}{c}-10.20 \\
( \pm 0.20)\end{array}$ & $\begin{array}{c}-12.70 \\
( \pm 0.16)\end{array}$ & $\begin{array}{c}-38.43 \\
( \pm 0.52)\end{array}$ \\
\hline
\end{tabular}

a 1-Melm obviously does not provide a trans and cis species. For the sake of simplicity the data for 1-Melm were filed to the trans datasets of PDLs $2,2 \mathrm{e} \& \mathbf{2 g} .{ }^{\mathrm{b}}\left[\mathrm{kcal} \mathrm{mol}{ }^{-1}\right] ;{ }^{\mathrm{c}}\left[\mathrm{cal} \mathrm{mol}^{-1}\right]$. 

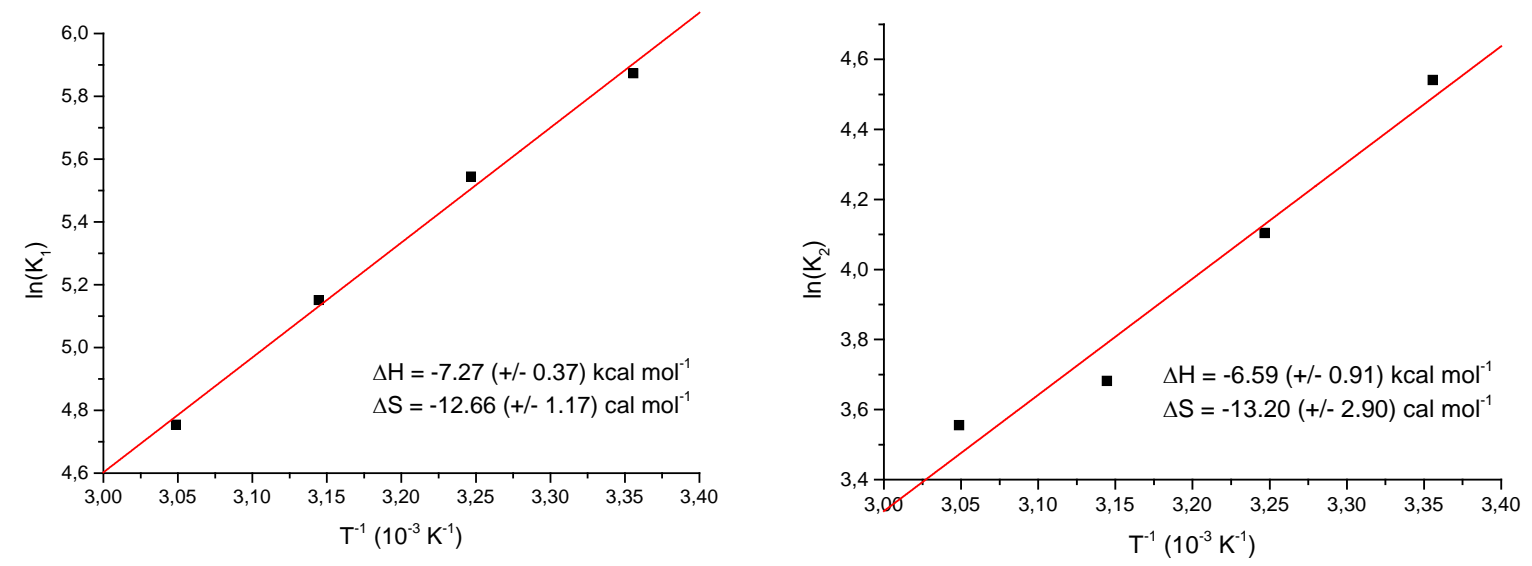

Figure S25: Arrhenius plots for determination of binding enthalpies and entropies for coordination of 1-methylimidazole to NiTPPF 20 .
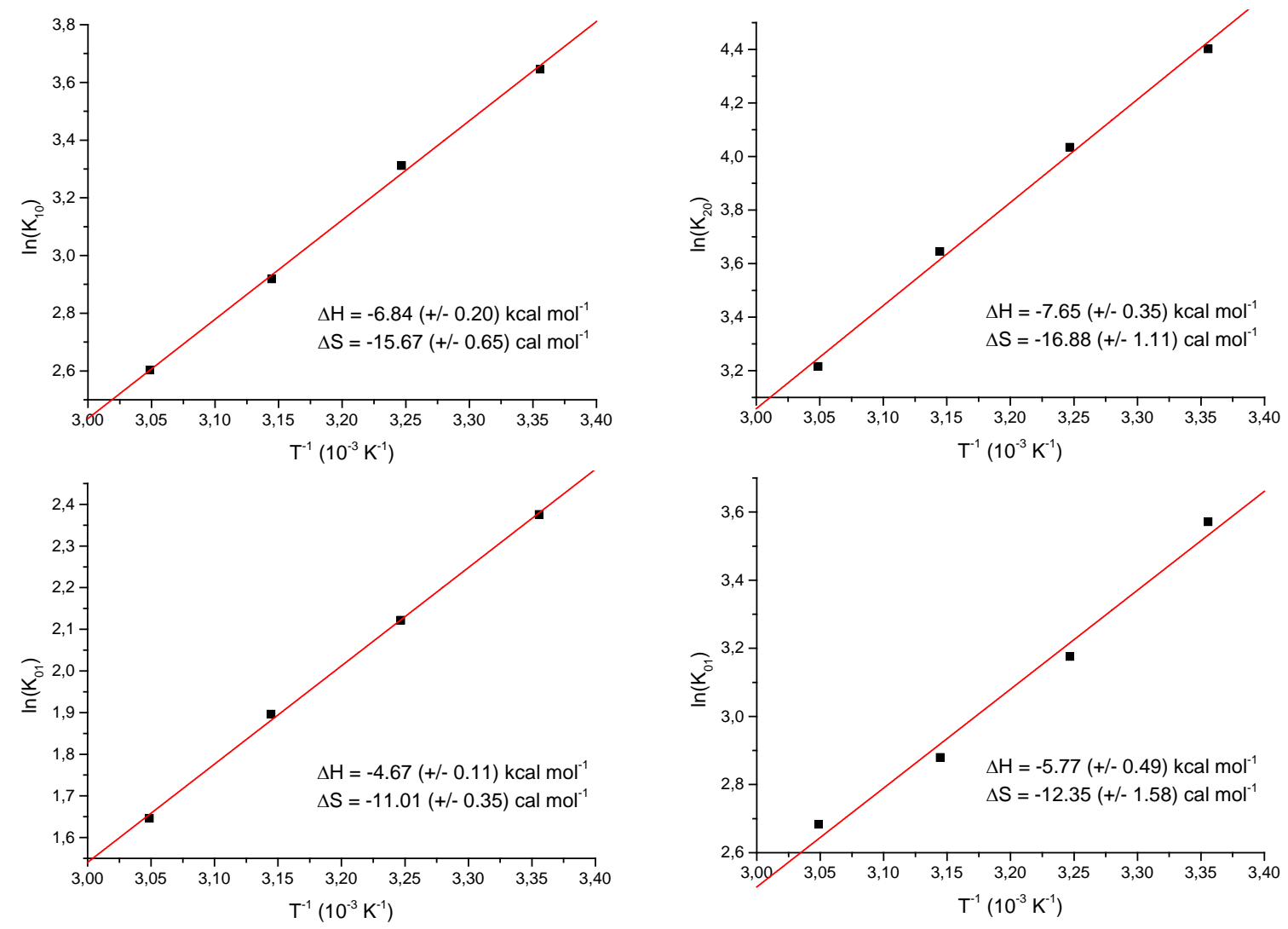

Figure S26: Arrhenius plots for determination of binding enthalpies and entropies for coordination of trans (top) and cis (down) 1-methyl-5-phenylazoimidazole 2 to NiTPPF 20. 

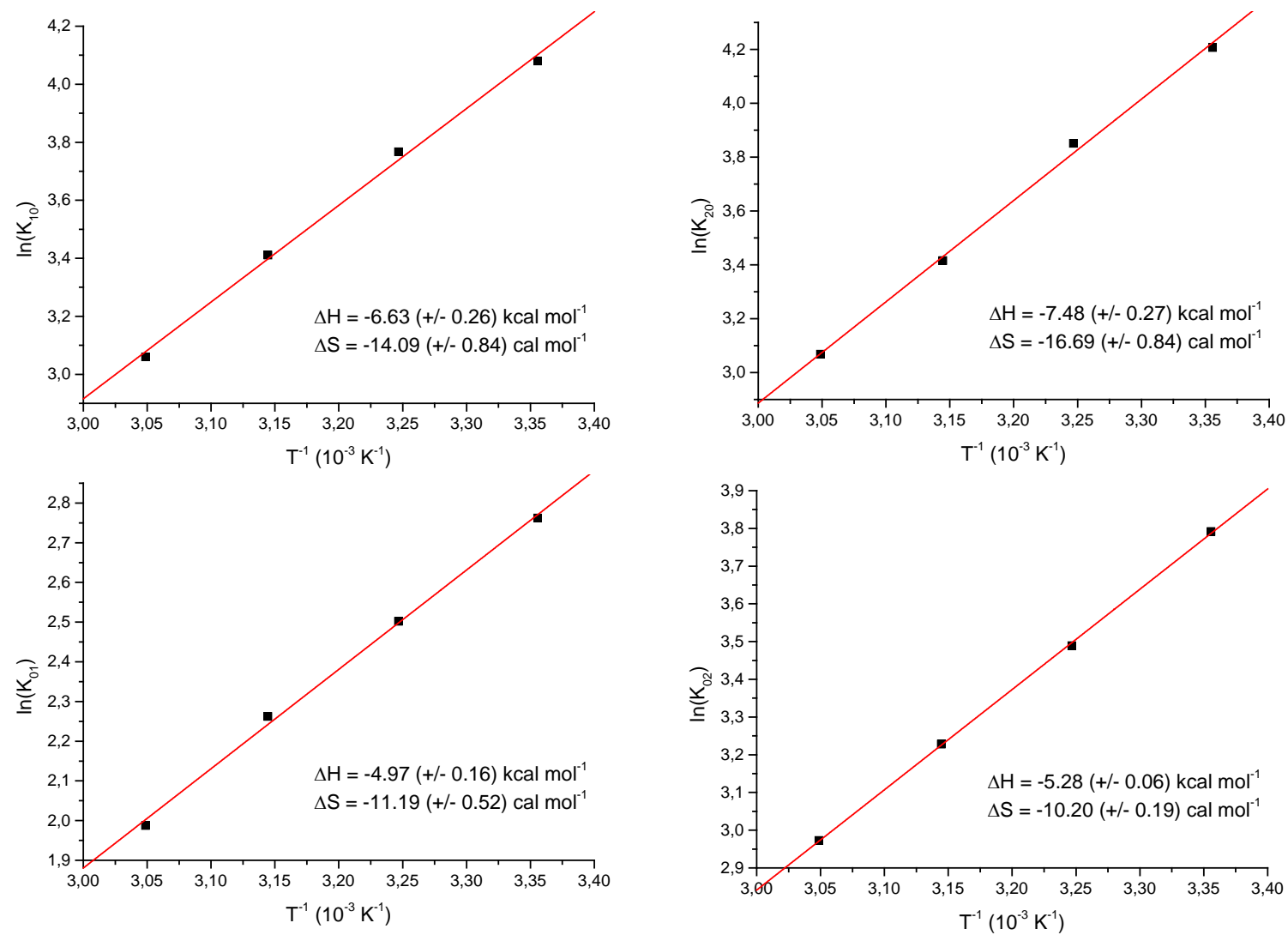

Figure S27: Arrhenius plots for determination of binding enthalpies and entropies for coordination of trans (top) and cis (down) 1-methyl-5-(4'-methoxyphenylazo)imidazole $\mathbf{2} \mathbf{b}$ to NiTPPF 20.
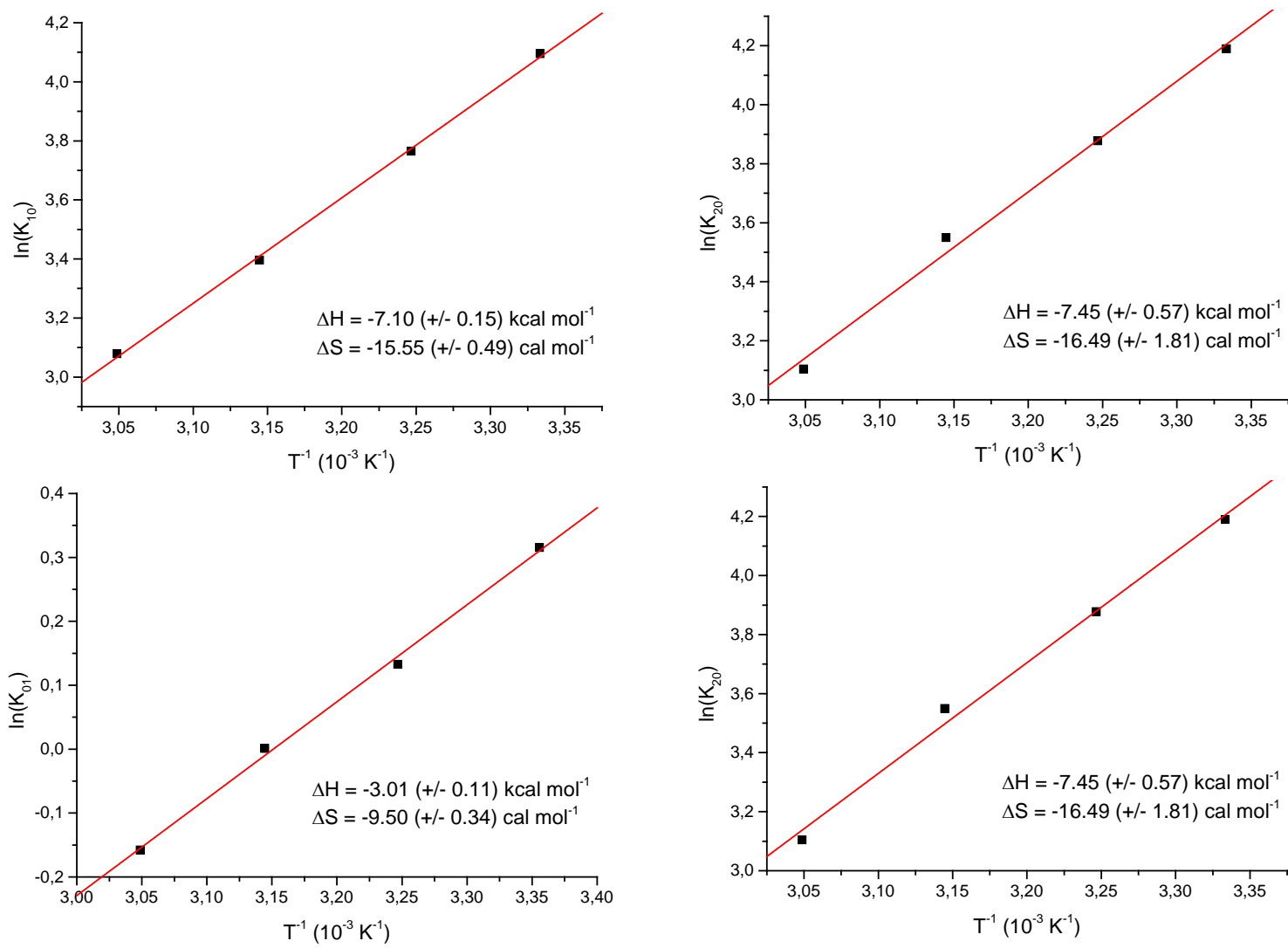

Figure S28: Arrhenius plots for determination of binding enthalpies and entropies for coordination of trans (top) and cis (down) 1-methyl-5-(3',5'-di-tert-butyl-4'-methoxyphenylazo)imidazole $2 \mathrm{e}$ to $\mathrm{NiTPPF}_{20}$. 


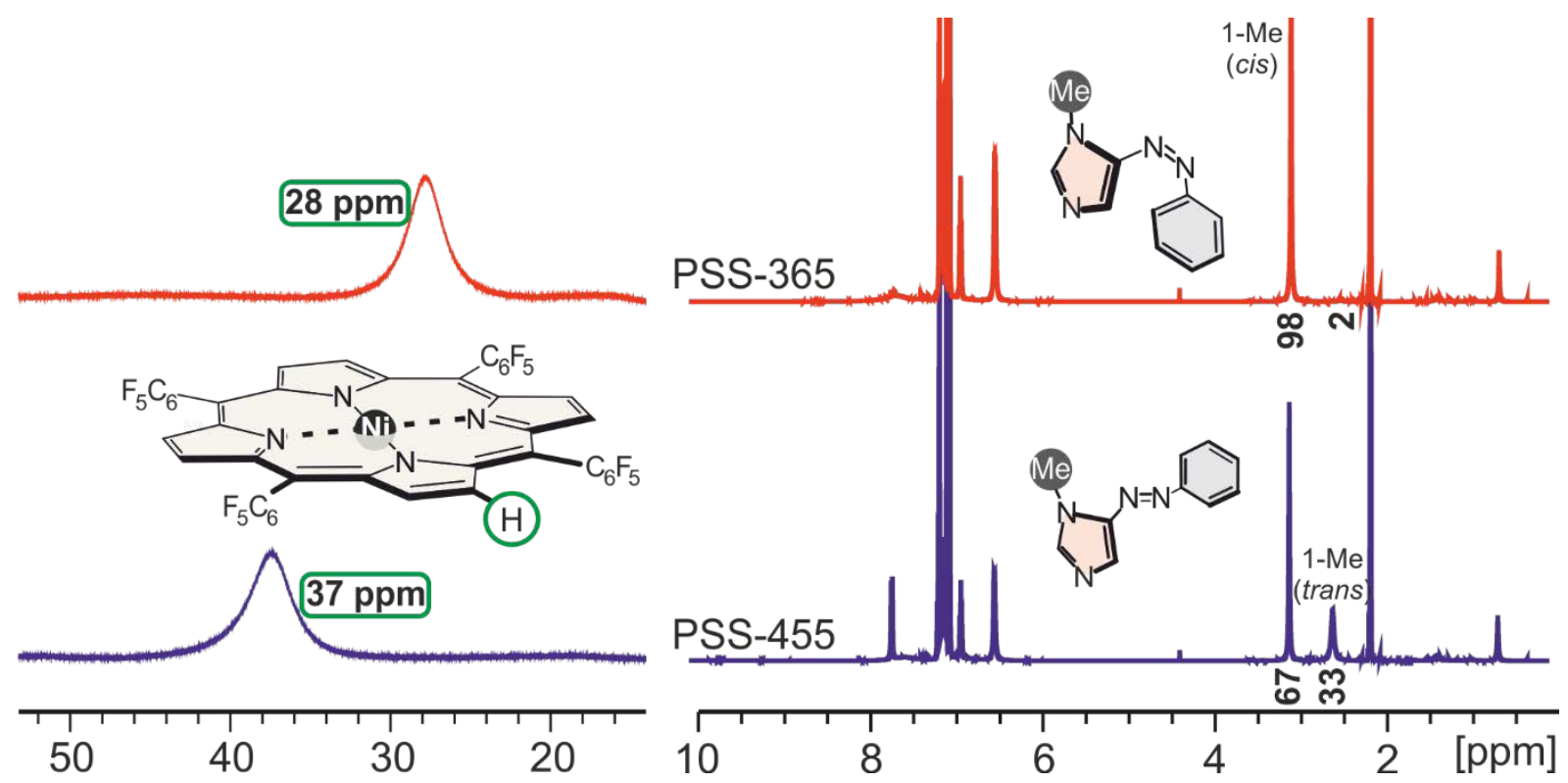

Figure S29: NMR switching experiments with phenylazoimidazole 2 (34.9 mM) and NiTPPF 20 (1.94 mM) in toluene- $d_{8}$. The amount of paramagnetic $\mathrm{Ni}^{2+}$ species in both photostationary states (PSS-365 and PSS-455) was calculated from the shift of the porphyrin's pyrrole protons (left). The corresponding $\mathrm{cis} /$ trans ratios were determined from the integrals of the 1-methyl group (right).

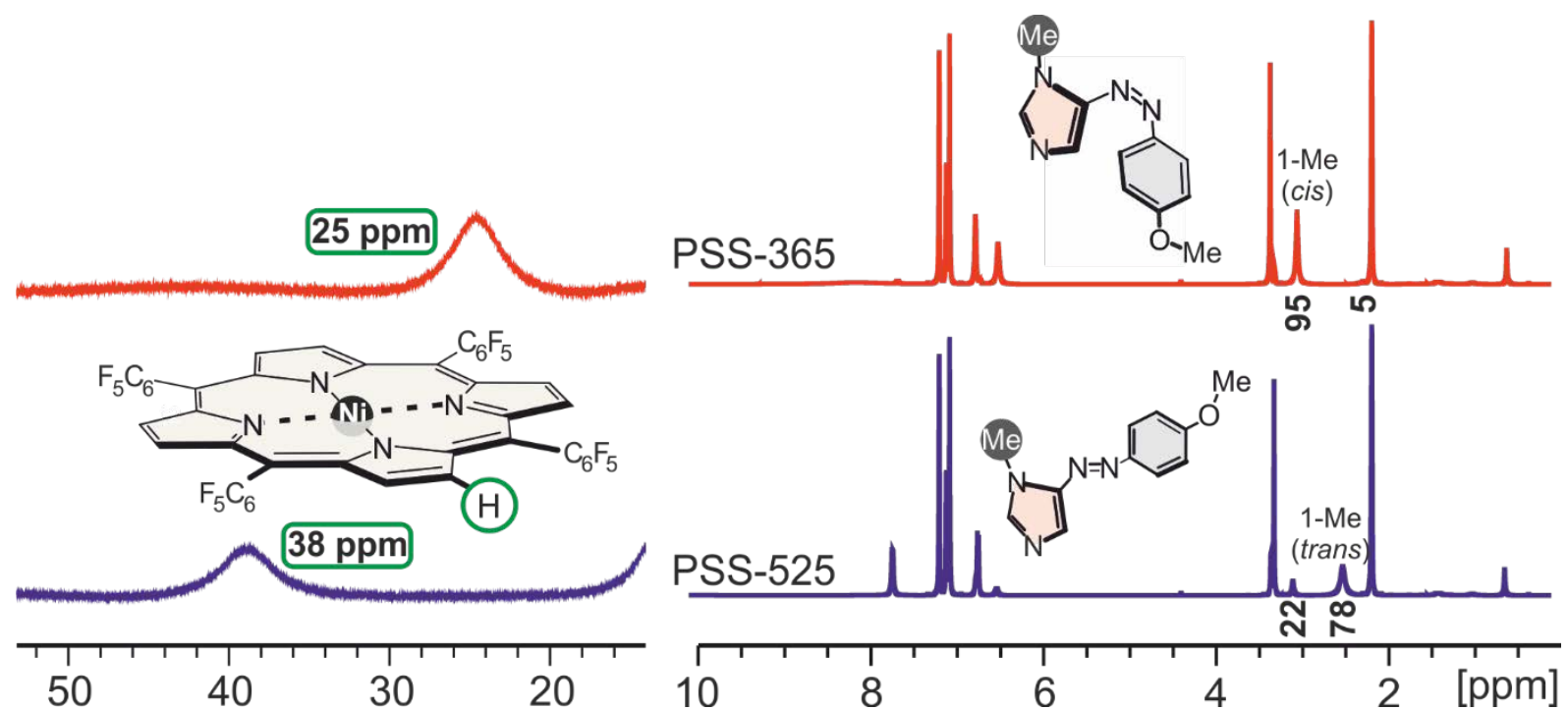

Figure S30: NMR switching experiments with phenylazoimidazole $\mathbf{2 b}(19.4 \mathrm{mM})$ and NiTPPF 20 (1.94 mM) in toluene- $\mathrm{d}_{8}$. The amount of paramagnetic $\mathrm{Ni}^{2+}$ species in both photostationary states (PSS-365 and PSS-525) was calculated from the shift of the porphyrin's pyrrole protons (left). The corresponding $\mathrm{cis} /$ trans ratios were determined from the integrals of the 1-methyl group (right). 


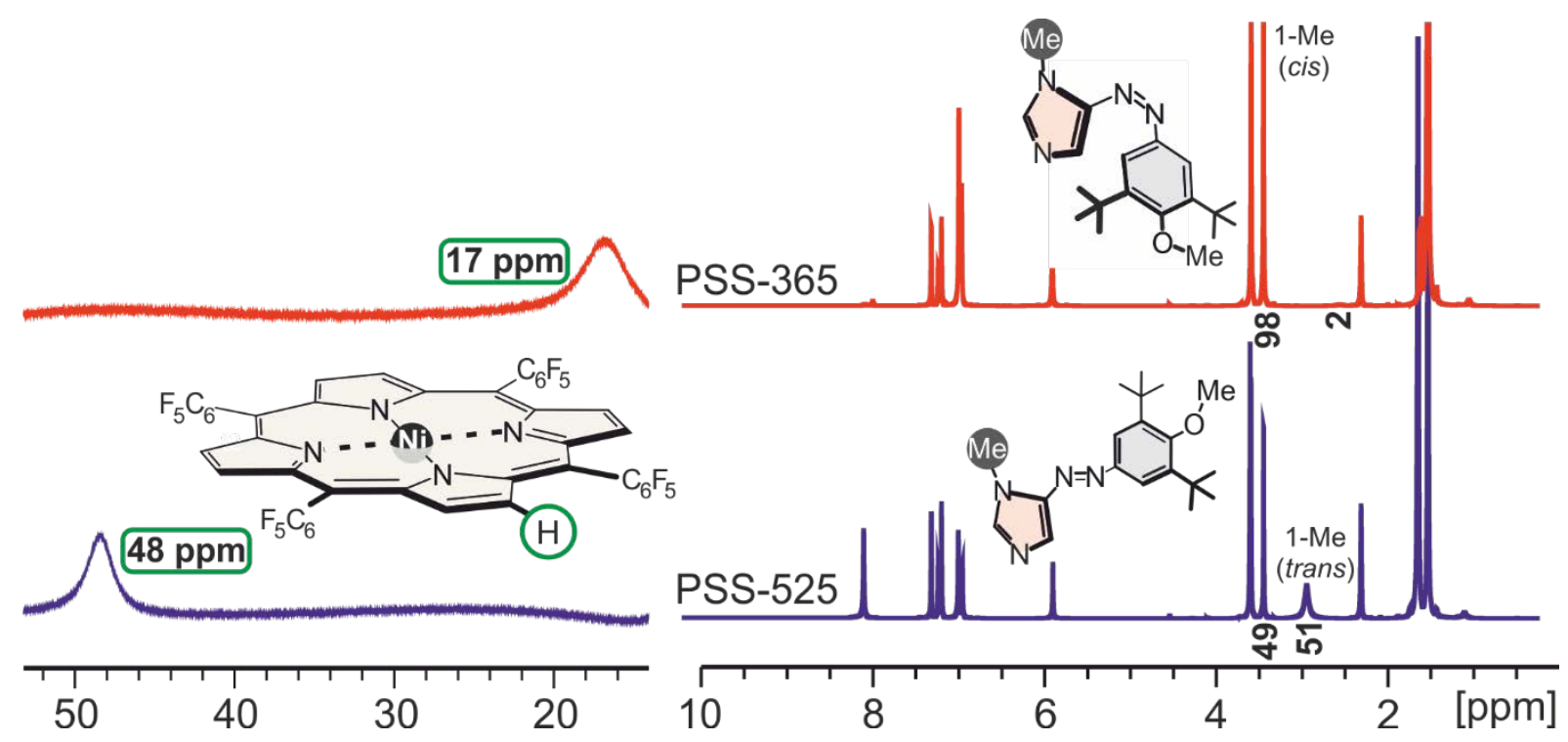

Figure S31: NMR switching experiments with phenylazoimidazole 2e (67.9 mM) and NiTPPF 20 (1.94 mM) in toluene- $d_{8}$. The amount of paramagnetic $\mathrm{Ni}^{2+}$ species in both photostationary states (PSS-365 and PSS-525) was calculated from the shift of the porphyrin's pyrrole protons (left). The corresponding cis/trans ratios were determined from the integrals of the 1-methyl group (right).

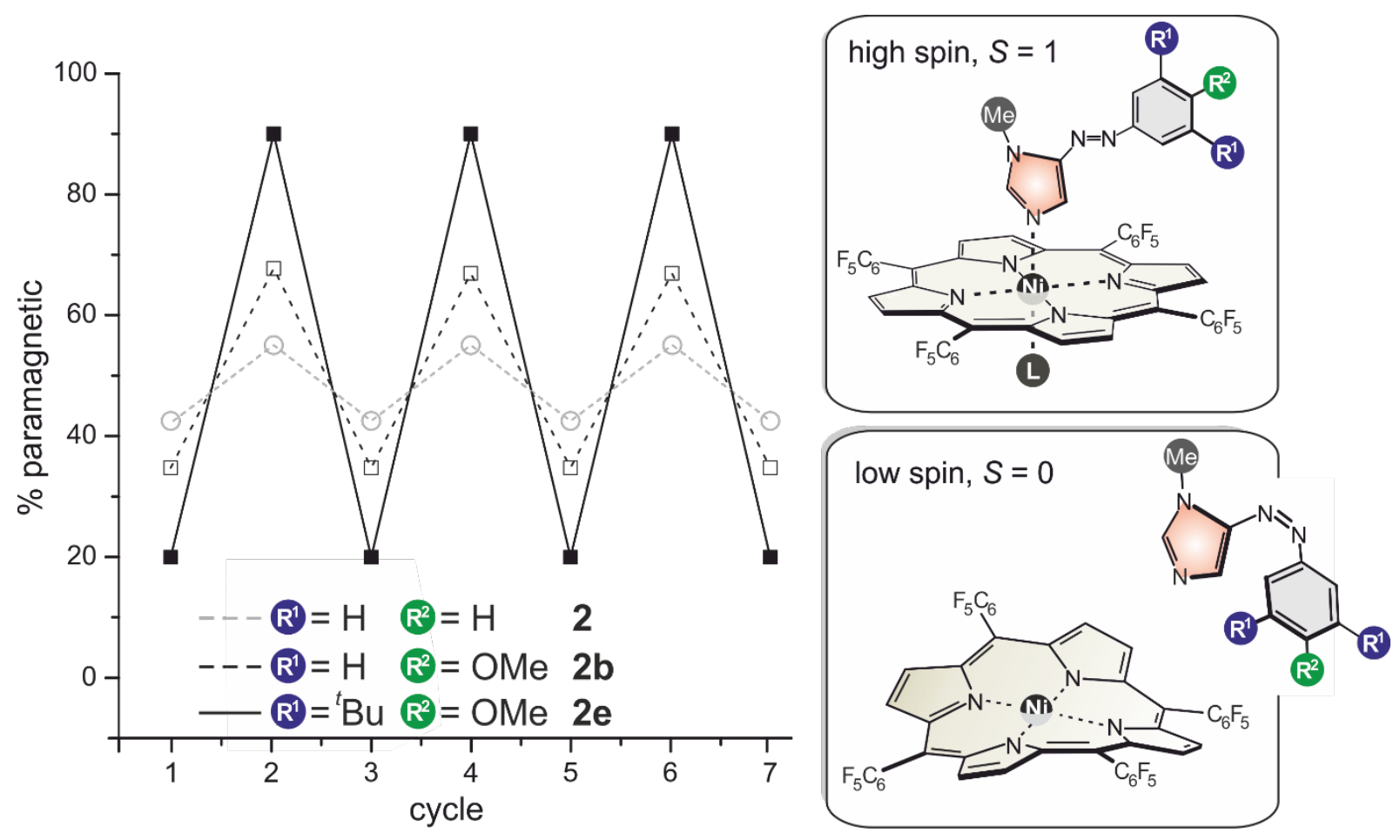

Figure S32: Reversible spin state switching of NiTPPF 20 with phenylazoimidazoles $\mathbf{2}$ (parent system), $\mathbf{2 b}$ (4'-methoxy derivative) and 2 e (3', 5'-di-tert-butyl-4'-methoxy derivative) in toluene- $\mathrm{d}_{8}$. The proportion of paramagnetic $\mathrm{Ni}^{2+}$ species is plotted as a function of the switching cycle. Switching is achieved by irradiation with $365 \mathrm{~nm}$ (trans $\rightarrow$ cis) and $455 \mathrm{~nm}$ for $\mathbf{2}$ or 525 for $\mathbf{2 b}$ and $\mathbf{2 e}$ (cis $\rightarrow$ trans). 


\section{Literature}

[1] TURBOMOLE V6.3 2011, a development of University of Karlsruhe and Forschungszentrum Karlsruhe GmbH, 1989-2007, TURBOMOLE GmbH, since 2007; available from http://www.turbomole.com.

[2] Gaussian 09, Revision D.01, Frisch, M. J.; Trucks, G. W.; Schlegel, H. B.; Scuseria, G. E.; Robb, M. A.; Cheeseman, J. R.; Scalmani, G.; Barone, V.; Mennucci, B.; Petersson, G. A.; Nakatsuji, H.; Caricato, M.; Li, X.; Hratchian, H. P.; Izmaylov, A. F.; Bloino, J.; Zheng, G.; Sonnenberg, J. L.; Hada, M.; Ehara, M.; Toyota, K.; Fukuda, R.; Hasegawa, J.; Ishida, M.; Nakajima, T.; Honda, Y.; Kitao, O.; Nakai, H.; Vreven, T.; Montgomery, J. A., Jr.; Peralta, J. E.; Ogliaro, F.; Bearpark, M.; Heyd, J. J.; Brothers, E.; Kudin, K. N.; Staroverov, V. N.; Kobayashi, R.; Normand, J.; Raghavachari, K.; Rendell, A.; Burant, J. C.; Iyengar, S. S.; Tomasi, J.; Cossi, M.; Rega, N.; Millam, J. M.; Klene, M.; Knox, J. E.; Cross, J. B.; Bakken, V.; Adamo, C.; Jaramillo, J.; Gomperts, R.; Stratmann, R. E.; Yazyev, O.; Austin, A. J.; Cammi, R.; Pomelli, C.; Ochterski, J. W.; Martin, R. L.; Morokuma, K.; Zakrzewski, V. G.; Voth, G. A.; Salvador, P.; Dannenberg, J. J.; Dapprich, S.; Daniels, A. D.; Farkas, Ö.; Foresman, J. B.; Ortiz, J. V.; Cioslowski, J.; Fox, D. J. Gaussian, Inc., Wallingford CT, 2009.

[3] Grimme, S.; Parac, M. ChemPhysChem 2003, 4, 292.

[4] Thies, S.; Sell, H.; Schütt, C.; Bornholdt, C.; Näther, C.; Tuczek, F.; Herges, R. J. Am. Chem. Soc. 2011, $133,16243-16250$.

[5] del Piero, S.; Melchior, A.; Polese, P.; Portanova, R.; Tolazzi, M. Annali di Chimica 2006, 96, 29-49. 